\title{
Statistical Modelling for Sports Scientists: Practical Introduction Using R (Part 1)

\author{
Mladen Jovanović
}

\section{Corresponding author}

Mladen Jovanović

Faculty of Sports and Physical Education

Blagoja Parovića 156

11030 Belgrade

Serbia

Email: coach.mladen.jovanovic@gmail.com

Twitter: physical_prep

Web: www.complementarytraining.net

This document is a pre-print

Version 6. Uploaded 17/10/2019

\section{Cite as:}

Jovanović, M. (2019). Statistical Modelling for Sports Scientists: Practical Introduction Using R (Part 1). SportRxiv. doi:10.31236/osf.io/dnq3m

DOI: $10.31236 /$ osf.io/dnq3m 


\begin{abstract}
The aim of this paper is to provide an overview of the three classes of tasks in the statistical modelling: description, prediction and causal inference (Hernán, Hsu \& Healy, 2019). Statistical inference is often required for all three tasks. Short introduction to frequentist null-hypothesis testing, Bayesian estimation and bootstrap are provided. Special attention is given to the practical significance with the introduction of magnitude-based estimators and statistical inference by using the concept of smallest effect size of interest (SESOI). Measurement error is discussed with the particular aim of interpreting individual change scores. In the second part of this paper, common sport science problems are introduced and analyzed with written package in R language, used for that purpose (RStudio Team, 2016; R Core Team, 2018).
\end{abstract}

Keywords: prediction, causal inference, Bayesian, individual-based, magnitude-based, equivalence, bootstrap, practical significance, measurement error 


\section{Contents}

$\begin{array}{lr}\text { Abstract } & 2\end{array}$

1 Introduction $\quad 5$

2 Three classes of tasks in the statistical modelling 6

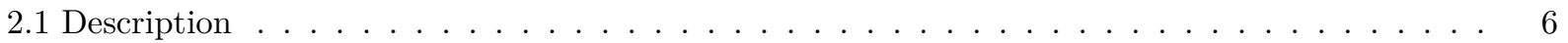

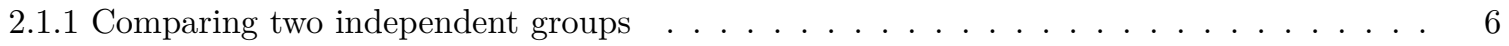

2.1.2 Comparing dependent groups . . . . . . . . . . . . . . . . . . . 15

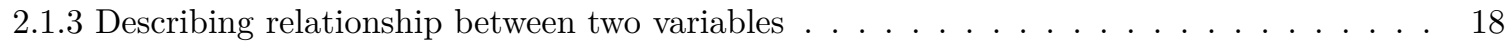

2.1 .4 Advanced uses . . . . . . . . . . . . . . . . . . . . . . 23

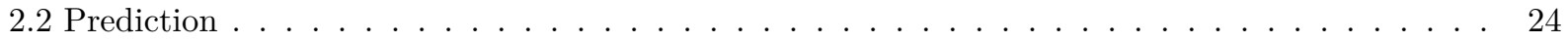

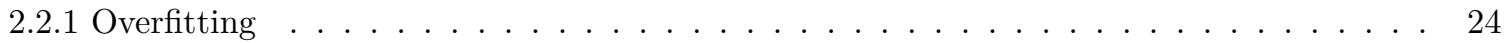

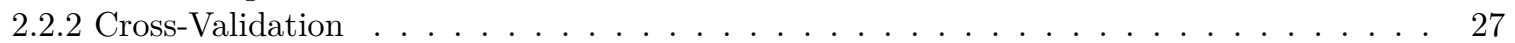

2.2 .3 Bias-Variance decomposition and trade-off . . . . . . . . . . . . . . . . . 28

2.2 .4 Interpretability . . . . . . . . . . . . . . . . . . . . . . . . . . 30

2.2.5 Magnitude-based prediction estimators . . . . . . . . . . . . . . . . . 31

2.3 Causal Inference . . . . . . . . . . . . . . . . . . . . . . . . . . . . 32

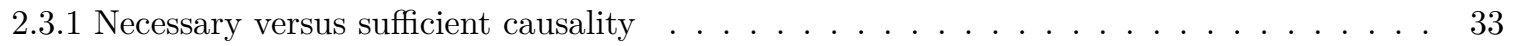

2.3 .2 Observational data . . . . . . . . . . . . . . . . . . . . . . . . 33

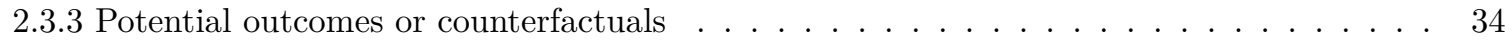

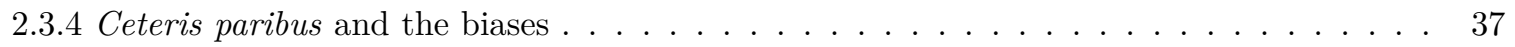

2.3 .5 Randomization . . . . . . . . . . . . . . . . . . . . 37

2.3.6 Subject matter knowledge . . . . . . . . . . . . . . . . . . . . . . . 39

2.3.7 Example of randomized control trial . . . . . . . . . . . . . . . . . 39

2.3.8 Prediction as a complement to causal inference . . . . . . . . . . . . . . . . . 44

2.3.9 Responders vs non-responders . . . . . . . . . . . . . . . . . . . . . 47

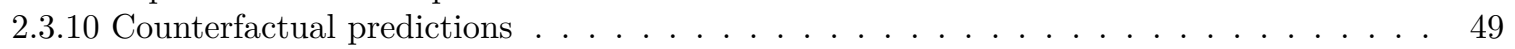

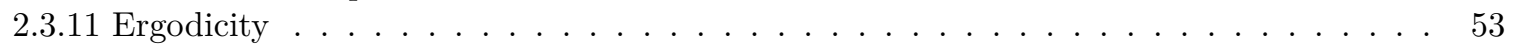

3 Statistical Inference $\quad \mathbf{5 4}$

3.1 Two kinds of uncertainty, two kinds of probability, two kinds of statistical inference . . . . . . 54

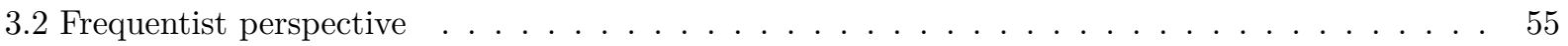

3.2.1 Null-Hypothesis Significance Testing . . . . . . . . . . . . . . . . . . . . 58

3.2 .2 Statistical Power . . . . . . . . . . . . . . . . . . . . . . . 60

3.2 .3 New Statistics: Confidence Intervals and Estimation . . . . . . . . . . . . . . . . . 62

3.2 .4 Minimum Effect Tests . . . . . . . . . . . . . . . . . . . . . . . . . . 64

3.2 .5 Magnitude Based Inference . . . . . . . . . . . . . . . . . . . . . 69

3.3 Bayesian perspective $\ldots \ldots \ldots \ldots \ldots \ldots \ldots \ldots \ldots$

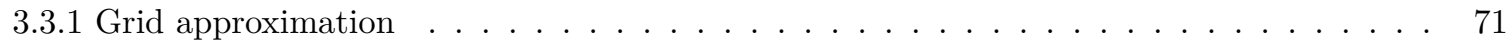

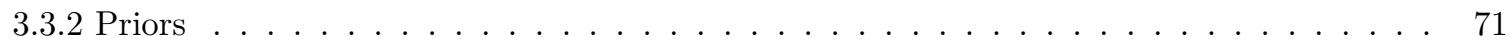

3.3 .3 Likelihood function . . . . . . . . . . . . . . . . . . . . . . . 72

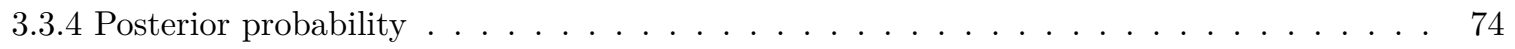

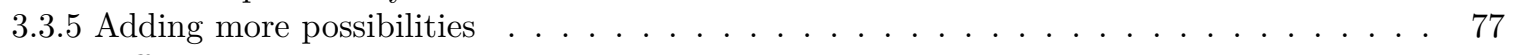

3.3 .6 Different prior . . . . . . . . . . . . . . . . . . . . . . . . . . 79

3.3 .7 More data . . . . . . . . . . . . . . . . . . . . . . . . 80

3.3.8 Summarizing prior and posterior distributions with MAP and HDI . . . . . . . . . . 81

3.3 .9 Comparison to NHST Type I errors . . . . . . . . . . . . . . . . . . 82

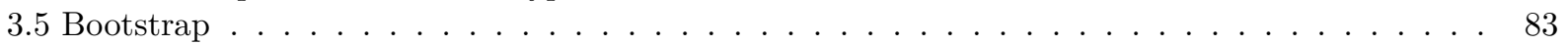

3.5 .1 Summarizing bootstrap distribution $\ldots \ldots \ldots \ldots \ldots \ldots \ldots$

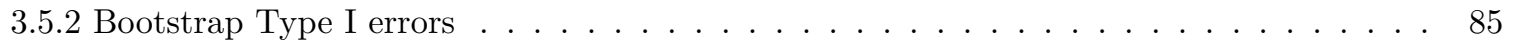

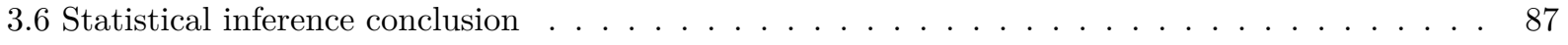

$\begin{array}{ll}4 \text { Measurement Error } & 88\end{array}$ 
4.1 Interpreting individual changes using SESOI and $\mathrm{TE} \ldots \ldots \ldots \ldots \ldots$

$\begin{array}{lr}5 \text { Conclusion } & 96\end{array}$

$\begin{array}{ll}\text { Recommended material } & 97\end{array}$

$\begin{array}{lr}\text { References } & 98\end{array}$ 


\section{Introduction}

The real world is very complex and uncertain. In order to help in understanding it and to predict its behavior, we create maps and models (Page, 2018; Weinberg \& McCann, 2019). One such tool are statistical models, representing a simplification of the complex and ultimately uncertain reality, in the hope of describing it, understanding it, predicting its behavior, and help in making decisions and interventions (McElreath, 2015; Lang, Sweet \& Grandfield, 2017; Pearl \& Mackenzie, 2018; Hernán, Hsu \& Healy, 2019). In the outstanding statistics book "Statistical Rethinking" (McElreath, 2015), the author stresses the distinction between Large World and Small World, described initially by Leonard Savage (Savage, 1972; Binmore, 2011; Gigerenzer, Hertwig \& Pachur, 2015):

"All statistical modeling has these same two frames: the small world of the model itself and the large world we hope to deploy the model in. Navigating between these two worlds remains a central challenge of statistical modeling. The challenge is aggravated by forgetting the distinction.

The small world is the self-contained logical world of the model. Within the small world, all possibilities are nominated. There are no pure surprises, like the existence of a huge continent between Europe and Asia. Within the small world of the model, it is important to be able to verify the model's logic, making sure that it performs as expected under favorable assumptions. Bayesian models have some advantages in this regard, as they have reasonable claims to optimality: No alternative model could make better use of the information in the data and support better decisions, assuming the small world is an accurate description of the real world.

The large world is the broader context in which one deploys a model. In the large world, there may be events that were not imagined in the small world. Moreover, the model is always an incomplete representation of the large world, and so will make mistakes, even if all kinds of events have been properly nominated. The logical consistency of a model in the small world is no guarantee that it will be optimal in the large world. But it is certainly a warm comfort." (p. 19)

Creating "Small Worlds" relies heavily on making and accepting numerous assumptions, both known and unknown, as well as prior expert knowledge, which is ultimately incomplete and fallible. Because all statistical models require subjective choices (Gelman \& Hennig, 2017), there is no objective approach to make "Large World" inferences. It means that it must be us who make the inference, and claims about the "Large World" will always be uncertain. Additionally, we should treat statistical models and statistical results as being much more incomplete and uncertain than the current norm (Amrhein, Trafimow \& Greenland, 2019).

We must accept the pluralism of statistical models and models in general (Mitchell, 2002, 2012), move beyond subjective-objective dichotomy by replacing it with virtues such as transparency, consensus, impartiality, correspondence to observable reality, awareness of multiple perspectives, awareness of context-dependence, and investigation of stability (Gelman \& Hennig, 2017). Finally, we need to accept that we must act based on cumulative knowledge rather than solely rely on single studies or even single lines of research (Amrhein, Trafimow \& Greenland, 2019).

This discussion is the topic of epistemology, scientific inference, and philosophy of science, thus far beyond the scope of the present paper (and the author). Nonetheless, it was essential to convey that statistical modeling is a process of creating the "Small Worlds" and deploying it in the "Large World". There are three main classes of tasks that the statistical model is hoping to achieve: description, prediction, and causal inference (Hernán, Hsu \& Healy, 2019).

The following example will help in differentiating between these three classes of tasks. Consider a king who is facing a drought who must decide whether to invest resources in rain dances. The queen, upon seeing some rain clouds in the sky, must decide on whether to carry her umbrella or not. Young prince, who likes to gamble during his hunting sessions, is interested in knowing what region of his father's vast Kingdom receives the most rain. All three would benefit from an empirical study of rain, but they have different requirements of the statistical model. The king requires causality: Do rain dances cause rain? The queen requires prediction: Does it look likely enough to rain for me to ask my servants to get my umbrella? The prince requires simple quantitative summary description: have I put my bets on the correct region? 
The following section will provide an overview of the three classes of tasks in the statistical modelling. Data can be classified as being on one of four scales: nominal, ordinal, interval or ratio and description, prediction and causal techniques differ depending on the scale utilized. For the sake of simplicity and big picture overview, only examples using ratio scale would be considered in this paper.

\section{Disclosures}

The code to reproduce both the analyses and the paper itself will be available via the Open Science Framework and GitHub repository once the paper is accepted to a peer-reviewed journal. The manuscript, including the figures, tables and statistical analyses, was created using RStudio (RStudio Team, 2016) and R (R Core Team, 2018) and the R packages tidyverse (Wickham, 2017), cowplot (Wilke, 2019), psych (Revelle, 2018), rmarkdown (Xie, Allaire \& Grolemund, 2018), knitr (Xie, 2014, 2015, 2018), kableExtra (Zhu, 2019), ggridges (Wilke, 2018), ggstance (Henry, Wickham \& Chang, 2019), minerva (Albanese et al., 2012), directlabels (Hocking, 2018), caret (Kuhn et al., 2018; Kuhn \& Johnson, 2018, 2019), iml (Molnar, Bischl \& Casalicchio, 2018), boot (Davison \& Hinkley, 1997; Canty \& Ripley, 2017), and bayestestR (Makowski, Ben-Shachar \& Lüdecke, 2019a,b; Makowski et al.).

\section{Three classes of tasks in the statistical modelling}

\subsection{Description}

Description provides quantitative summary of the acquired data sample. These quantitative summaries are termed descriptive statistics or descriptive estimators and are usually broken down into two main categories: measures of central tendency, and measures of spread / dispersion. The stance taken in this paper is that descriptive statistics involve all quantitative summaries (or aggregates) that are used to describe data without making predictive or causal claims. For example, linear regression between two variables can be used as a descriptive tool if the aim is to measure linear association between two variables, but it can be also used in predictive and causal tasks. Effect sizes such as change, percent change or Cohen's $d$ represent descriptive statistics used to compare two or more groups, and are commonly used in causal tasks to estimate average causal effect of the treatment.

To provide further explanation of the descriptive statistics, three common descriptive tasks in sport science are given as examples: (1) comparing two independent groups, (2) comparing two dependent groups, (3) measuring association between two variables.

\subsubsection{Comparing two independent groups}

Imagine we carried collection of body height measurements and we obtained $\mathrm{N}=100$ observations using $\mathrm{N}=50$ female and $\mathrm{N}=50$ male subjects. Collected data is visualized in Figure 1. 

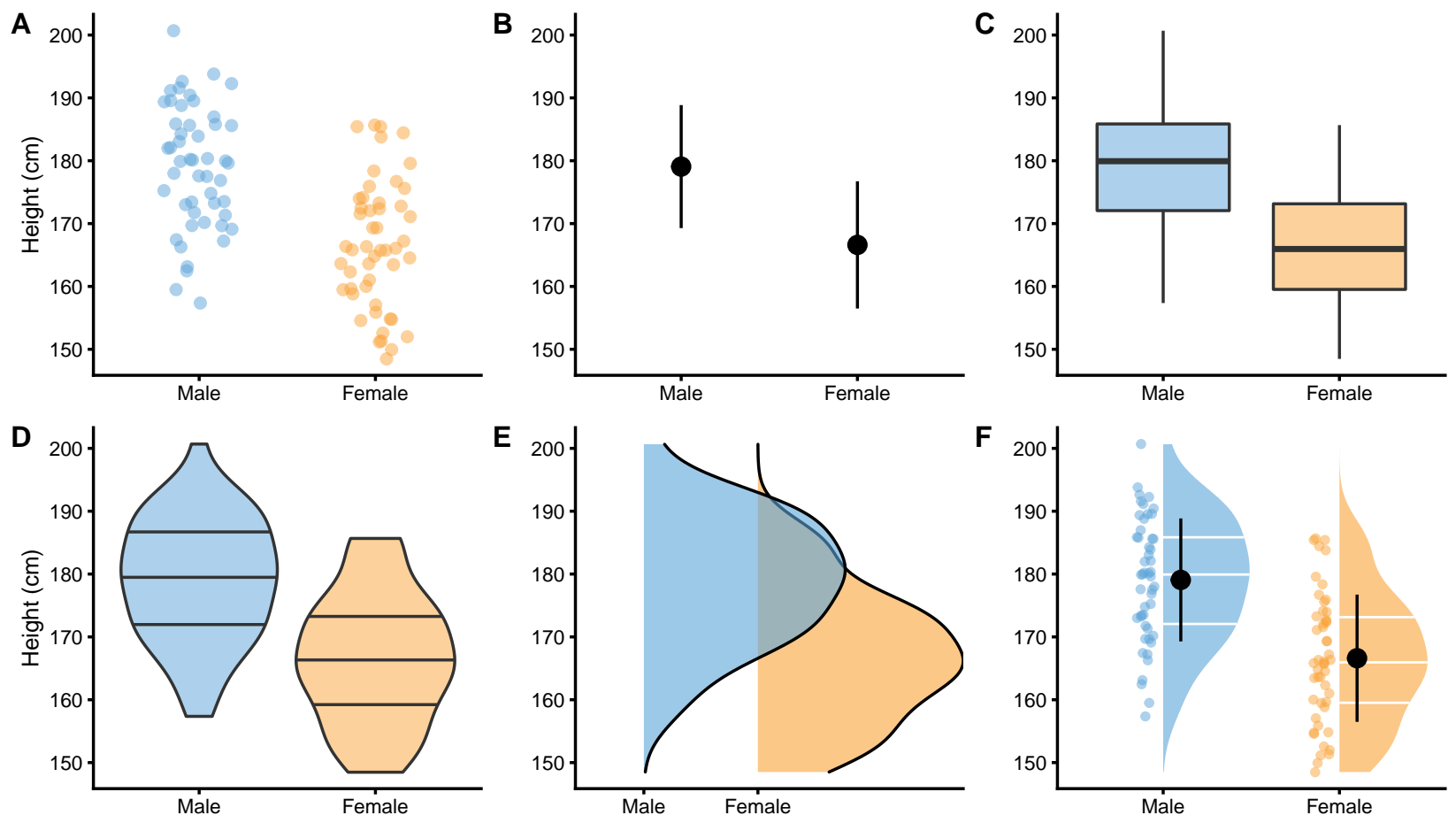

Figure 1. Common techniques to visualize independent groups observations. Before any analysis takes place, it is always a good practice to visualize the data first. Ideally, we want to visualize the complete data set, rather than only provide descriptive summaries, such as means. A. Simple scatter-plot with jitter to avoid overlap between the points. B. Mean and standard deviation as error bars. C. Box-plot.

Horizontal line represents median, or 50th percentile, whereas boxes represent 25 th and 75 th percentile. Vertical lines usually represent min and max, although they can extend up to $1.5 \mathrm{xIQR}$ (inter-quartile range) with point outside of that interval plotted as outliers. D. Violin plots representing double-side density plots with 25 th, 50 th and 75 th percentile lines. E. Density plots indicating sample distribution. F. Raincloud plot (Allen et al., 2018, 2019) which combine kernel density plots as clouds with accompanying 25th, 50th and 75 th percentile lines, mean \pm SD error bars and jittered points as rain

Commonly provided descriptive statistics for each group can be found in Table 1. Mean, median and mode are common measures of central tendencies. Standard deviation (SD), median absolute difference (MAD), inter-quartile range (IQR), min, max and range are common measures of spread or dispersion. Percent coefficient of variation $(\% \mathrm{CV})$ is also a measure of dispersion, but standardized ${ }^{1}$ which allows comparison of variables that are on different scales. Skewness (skew) is usually described as a measure of a symmetry. A perfectly symmetrical data set will have a skewness of 0 . Kurtosis measures the tail-heaviness of the distribution. More in depth discussion of descriptive statistics, particularly robust estimators (Wilcox, 2016; Wilcox \& Rousselet, 2017; Rousselet, Pernet \& Wilcox, 2017; Wilcox, Peterson \& McNitt-Gray, 2018) is beyond the topic of this short overview.

Table 1. Common descriptive statistics or estimators

\begin{tabular}{|c|c|c|c|c|c|c|c|c|c|c|c|c|c|}
\hline Gender & $\mathrm{n}$ & mean $(\mathrm{cm})$ & $\mathrm{SD}(\mathrm{cm})$ & $\% \mathrm{CV}$ & median $(\mathrm{cm})$ & MAD (cm) & IQR $(\mathrm{cm})$ & mode $(\mathrm{cm})$ & $\min (\mathrm{cm})$ & $\max (\mathrm{cm})$ & range $(\mathrm{cm})$ & skew & kurtosis \\
\hline Male & 50 & 179.07 & 9.79 & 5 & 179.93 & 10.36 & 13.79 & 180.57 & 157.36 & 200.68 & 43.33 & -0.15 & -0.67 \\
\hline Female & 50 & 166.61 & 10.12 & 6 & 165.95 & 10.34 & 13.63 & 166.69 & 148.47 & 185.67 & 37.20 & 0.13 & -0.85 \\
\hline
\end{tabular}

\footnotetext{
${ }^{1}$ Standardization is the process of putting different variables on the same scale. This allows for easier comparison, as well as graphing using a common axis. For example, variables are usually standardized by using Z-Score $\left(z_{i}=\frac{x_{i}-\bar{x}}{S D_{x}}\right)$ which has a mean of zero and a standard deviation of 1 .
} 


\subsubsection{Sample mean as the simplest statistical model}

In the introduction of this paper, statistical models are defined as "Small Worlds" or simplifications of the complex and uncertain reality. From this perspective, sample mean can be considered the simplest statistical model. With this estimator we are representing all of the data points with one quantitative summary. However, how do we choose an estimate that represents the sample the best? Estimate that has the minimal error is selected as the optimal representative. Error is defined using a loss function that penalizes difference between the model estimate $\left(\hat{y}_{i}\right)$ and observations $\left(y_{i}\right)$, or the residuals. One such loss function is RMSE or root-mean-square-error (equation 1). RMSE thus represents a measure of the model fit, or how good the model fits the data ${ }^{2}$. Lower RMSE means lower error and thus a better fit.

$$
\begin{aligned}
\text { Loss funtion } & =f(\text { observed }, \text { predicted }) \\
R M S E & =\sqrt{\frac{1}{n} \sum_{i=1}^{n}\left(y_{i}-\hat{y}_{i}\right)^{2}}
\end{aligned}
$$

By using body height data from the female group, we can search for a body height estimate that minimizes the RMSE (Figure 2). That body height estimate would be considered the best representative of the sample, and thus the simplest statistical model.
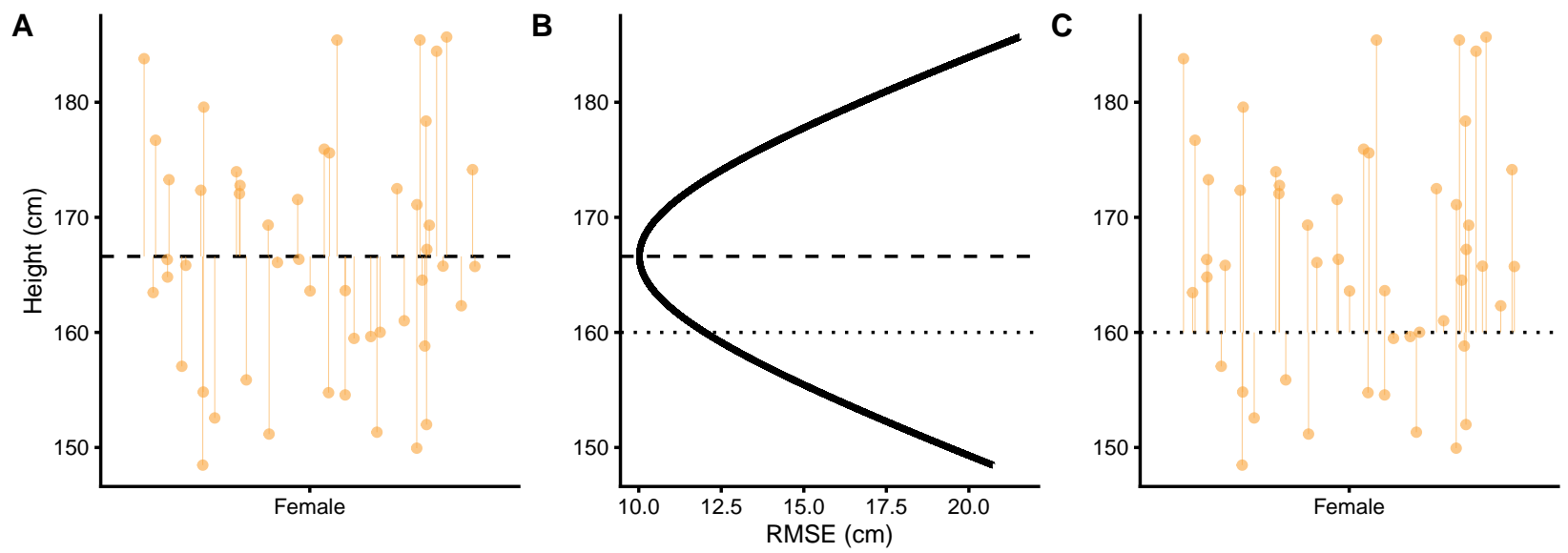

Figure 2. Sample mean as the simplest statistical model. A. Dashed line represents the estimate, in this case the mean of the sample. Vertical line represent residuals between estimate and observed values. B. Each estimate has a RMSE value. Central tendency estimate with the lowest RMSE value is the sample mean. C. Similar to panel A, this panel depicts residuals for a central tendency estimate with higher RMSE

As the result of this search, the body height estimate that minimizes the error is $166.61 \mathrm{~cm}$, and accompanying RMSE is equal to $10.02 \mathrm{~cm}$. As it can be read from the Table 1, this optimal body height estimate is equal to calculated sample mean. Standard deviation of the sample is equal to RMSE ${ }^{3}$. From statistical modelling perspective, sample mean can be considered sample estimate that minimizes the sample SD, and sample SD can be seen as the measure of the model fit.

This search for the optimal estimate that minimizes the loss function can be expanded to other statistical models. For example, linear regression can be seen as a search for the line that minimizes RMSE (or residual

\footnotetext{
${ }^{2}$ In statistical modelling there are other loss functions that can be utilized, for example: mean absolute error (MAE) which is calculated using $\frac{1}{n} \sum_{i=1}^{n}\left|y_{i}-\hat{y}_{i}\right|$ equation.

${ }^{3}$ As can be noticed, RMSE and SD are not exactly the same. This is because a sample SD equation uses $n-1$ instead of $n$ : $S D=\sqrt{\frac{1}{n-1} \Sigma_{i=1}^{n}\left(y_{i}-\bar{y}\right)^{2}}$, where $\bar{y}$ represents the mean. Remember that $\hat{y}_{i}$ represents the model estimate. In this case model estimate $\hat{y}_{i}$ and sample mean $\bar{y}$ are the same. Sample SD uses $n-1$ since this represents unbiased estimator of the population SD. More about this topic will be covered in "Statistical Inference" section.
} 
standard error). This approach of estimating model parameters or estimators belongs to the family of ordinary least squares (OLS) methods, although there are other approaches such as maximum likelihood estimation (MLE) which will be discussed in "Statistical Inference" section (Foreman, 2014). The solutions to some of these models can be found analytically ${ }^{4}$, but for some there is no analytical solution and computational approaches must be utilized. These computation approaches are referred to as optimization algorithms. The example given here involves only one parameter that needs to be optimized, in this case body height estimate, but real-life problems involve numerous parameters. The simple search through parameters state-space would take forever when it comes to problems involving more than only a few parameters. Algorithms that solve this computational problems are numerous, out of which the most popular ones are gradient descent, and Markov Chain Monte-Carlo (MCMC), which is utilized in Bayesian inference (will be discussed later on).

The take-home message from this short interlude is that even the simple descriptive statistics can be seen as statistical models.

\subsubsection{Effect Sizes}

Besides describing groups, we are often interested in comparing them. In order to achieve this task, a collection of estimators termed effect size statistics is utilized. Effect size can be defined in a narrow sense or in a broad sense. Briefly, the narrow sense refers to a family of standardized measures such as Cohen's $d$, while the broad sense refers to any measure of interest, standardized or not. The approach to effect size statistics in this paper is thus in a broad sense of the definition, in which all group comparison estimators are considered effect sizes statistics. In order to estimate effect sizes, one group needs to be considered baseline or control. The most common effect size statistics can be found in the Table 2 where female body height is considered baseline and compared with male body height.

Table 2. Effect size statistics for estimating differences between two independent groups

\begin{tabular}{rrrrrrrr}
\hline Difference $(\mathrm{cm})$ & SDdiff $(\mathrm{cm})$ & \% CVdiff & \% Difference & Ratio & Cohen's d & CLES & OVL \\
\hline 12.46 & 14.08 & 113 & 7 & 1.07 & 1.25 & 0.81 & 0.53 \\
\hline
\end{tabular}

Difference, or mean difference is calculated by subtracting group means. Using body height as an example, the mean difference between males and females is calculated by using the following equation (2):

$$
\begin{aligned}
\text { mean }_{\text {difference }} & =\text { mean }_{\text {males }}-\text { mean }_{\text {females }} \\
\text { mean }_{\text {males }} & =\frac{1}{n} \sum_{i=1}^{n} \text { male }_{i} \\
\text { mean }_{\text {females }} & =\frac{1}{n} \sum_{i=1}^{n} \text { female }_{i}
\end{aligned}
$$

$\%$ CVdiff, or percent coefficient of variation of the difference is the standard deviation of the difference (SDdiff - explained shortly) divided by mean difference (equation 3):

$$
\% C V_{\text {difference }}=100 \times \frac{S D_{\text {difference }}}{\text { mean }_{\text {difference }}}
$$

$\%$ Difference, or mean percent difference is calculated by dividing mean difference with the mean of the baseline group, in this case the female group, multiplied by 100 (equation 4 ):

$$
\text { mean }_{\% \text { difference }}=100 \times \frac{\text { mean }_{\text {difference }}}{\text { mean }_{\text {females }}}
$$

\footnotetext{
${ }^{4}$ The analytical solution for the estimate that minimizes SD is, of course, the sample mean $\left(\frac{1}{n} \sum_{i=1}^{n} y_{i}\right)$.
} 
Mean ratio, as its name suggests, is simple ratio between the two means (equation 5):

$$
\text { mean }_{\text {ratio }}=\frac{\text { mean }_{\text {males }}}{\text { mean }_{\text {females }}}
$$

Cohen's $d$ represent standardized effects size and thus preferable effect size statistic. For this reason, Cohen's $\mathrm{d}$ is commonly written as ES, short of effect size. Cohen's d for the independent groups is calculated by dividing mean difference with pooled standard deviation (equation 6):

$$
\begin{aligned}
\text { Cohen's } d & =\frac{\text { mean }_{\text {difference }}}{S D_{\text {pooled }}} \\
S D_{\text {pooled }} & =\sqrt{\frac{\left(n_{\text {males }}-1\right) S D_{\text {males }}^{2}+\left(n_{\text {females }}-1\right) S D_{\text {females }}^{2}}{n_{\text {males }}+n_{\text {females }}-2}}
\end{aligned}
$$

Why Cohen's d should be used instead of other effect size estimators can be demonstrated by a simple example, coming from a study by Buchheit and Rabbani (Buchheit \& Rabbani, 2014). In this study, authors examined the relationship between the performance in the Yo-Yo Intermittent Recovery Test Level 1 (Yo-YoIR1) and the 30-15 Intermittent Fitness Test (30-15IFT), and compared the sensitivity of both tests to the training. Although this study used two dependent groups (Pre-training and Post-training), the rationale can be applied to the topic of estimating effect sizes between the two independent groups. Table 3 contains Pre-training results and the effect sizes estimated with percent change ${ }^{5}$ and Cohen's d.

Table 3. Training intervention effect sizes for Yo-YoIR1 and 30-15IFT. Modified from Buchheit \& Rabbani, 2014

\begin{tabular}{llll}
\hline Test & Pre-training & \% Change & Cohen's d \\
\hline Yo-YoIR1 & $1031 \pm 257 \mathrm{~m}$ & $35 \%$ & 1.2 \\
30-15IFT & $17.4 \pm 1.1 \mathrm{kmh}^{-1}$ & $7 \%$ & 1.1 \\
\hline
\end{tabular}

Since Yo-YoIR1 and 30-15IFT utilize different scales (total meters covered and velocity reached respectively), percent change estimator is not a good choice to compare the effect sizes between the two tests ${ }^{6}$. Since Cohen's d is standardized estimator, it should be used when comparing tests or measures that are at different scales.

After estimating effect sizes, the question that naturally follows up is the question of magnitude. In other words - "how big is the effect?". Since Cohen's d is standardized estimator, it allows for establishment of qualitative magnitude thresholds. Based on the original work by Cohen (Cohen, 1988), Hopkins (Hopkins, 2006; Hopkins et al., 2009) suggested the following magnitudes of effect (Table 4). According to the Table 4, the difference in the mean body height between males and females would be considered large, as well as changes in both Yo-YoIR1 and 30-15IFT.

Table 4. Magnitudes of effect

\begin{tabular}{lllllll}
\hline Magnitude of effect & Trivial & Small & Moderate & Large & Very Large & Nearly Perfect \\
\hline Cohen's d & $0-0.2$ & $0.2-0.6$ & $0.6-1.2$ & $1.2-2.0$ & $2.0-4.0$ & $>4.0$ \\
\hline
\end{tabular}

\footnotetext{
${ }^{5}$ Percent change is the same estimator as percent difference, but applied to difference between the two dependent groups (see section "Comparing dependent groups")

${ }^{6}$ However, let's admit that we would rather report estimators of higher value, particularly if we are biased toward a specific test.
} 
Cohen's d, as well as associated magnitudes of effect, are commonly hard to interpret by non-statistically trained professionals (e.g. coaches). McGraw et al. (McGraw \& Wong, 1992) suggested common language effect size (CLES) estimator instead, which could be more intuitive to understand. CLES represents the probability that an observation sampled at random from one group will be greater than an observation sampled at random from other group. For example, if we take random male and random female from our two groups and repeat that 100 times $^{7}$, how many times a male would be taller than a female (Figure 3 )?
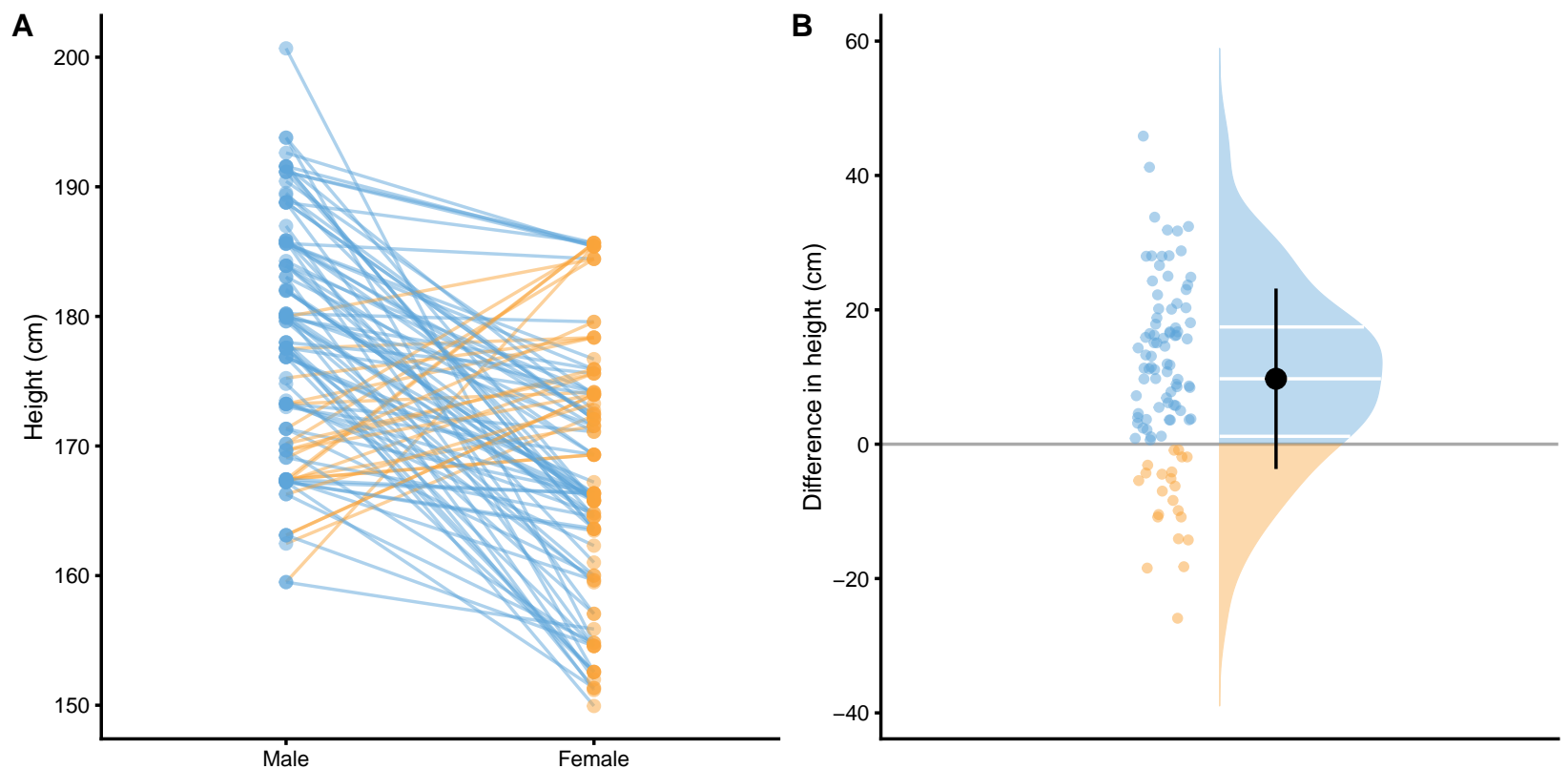

Figure 3. Drawing random 100 pairs to estimate probability of males being taller than

females. A. Scatterplot of 100 pairs drawn at random from two samples. Since we are comparing paired males and females, lines can be drawn between each of 100 draws. Blue line indicates taller male, while orange line indicates taller female. B. Distribution of the difference between males and females for each of 100 pairs drawn

By using simple counting from 100 random paired samples, males are taller in 78 cases, or $78 \%$. By using probability, that is equal to 0.78 . In other words, if I blindfoldedly, randomly select a male and a female from the two groups and if I bet that the male is taller, I would be correct $78 \%$ of the time.

CLES can be estimated using brute-force computational method, or algebraic method. Brute-force method involves generating all possible pair-wise combinations from two groups, and in our example that is equal to $50 \times 50=2500$ cases, and then simply counting in how many cases males are taller than females. This method can become very computationally intensive for groups with large sample number. Algebraic method, on the other hand, assumes normal distribution of the observations in the groups, and estimates standard deviation of the difference (equation 7). Note that standard deviation of the all pairwise differences estimated with brute-force method would be very similar to algebraically derived standard deviation of the difference.

$$
S D_{\text {difference }}=\sqrt{S D_{\text {males }}^{2}+S D_{\text {females }}^{2}}
$$

Algebraically, CLES is then derived from normal distribution (where mean of the distribution is equal to mean difference between the groups, and standard deviation of the distribution is equal to standard deviation

\footnotetext{
${ }^{7}$ In other words, we are drawing 100 paired samples from the two independent groups. This makes the drawn 100 observations paired or dependent.
} 
of the difference) by calculating probability of the difference scores higher than zero (see Figure 3B for a visual representation). Table 2 contains algebraically computed CLES estimate.

CLES equivalent is utilized as a performance metric in class prediction tasks, termed area under curve (AUC), where 0.5 is a predictive performance equal to a random guess, and 1 is perfect predictive separation between the two classes (James et al., 2017; Kuhn \& Johnson, 2018).

Overlap (OVL) estimator represents the overlap between the two sample distributions. Providing that samples are equal, the OVL is equal to 1 . Providing there is complete separation between the two samples, then OVL is equal to 0 (Figure 4A). OVL can be estimated with brute-force computational methods (which doesn't make assumptions regarding sample distribution) and with algebraic methods that make normality assumptions.

Since Cohen's d, CLES and OVL are mathematically related, it is possible to convert one to another (assuming normal distribution of the samples and equal SD between the two groups for the OVL estimation). Figure 4B depicts relationship between the Cohen's d, CLES, and OVL. Figure 4C depicts relationship between the CLES and OVL.

A

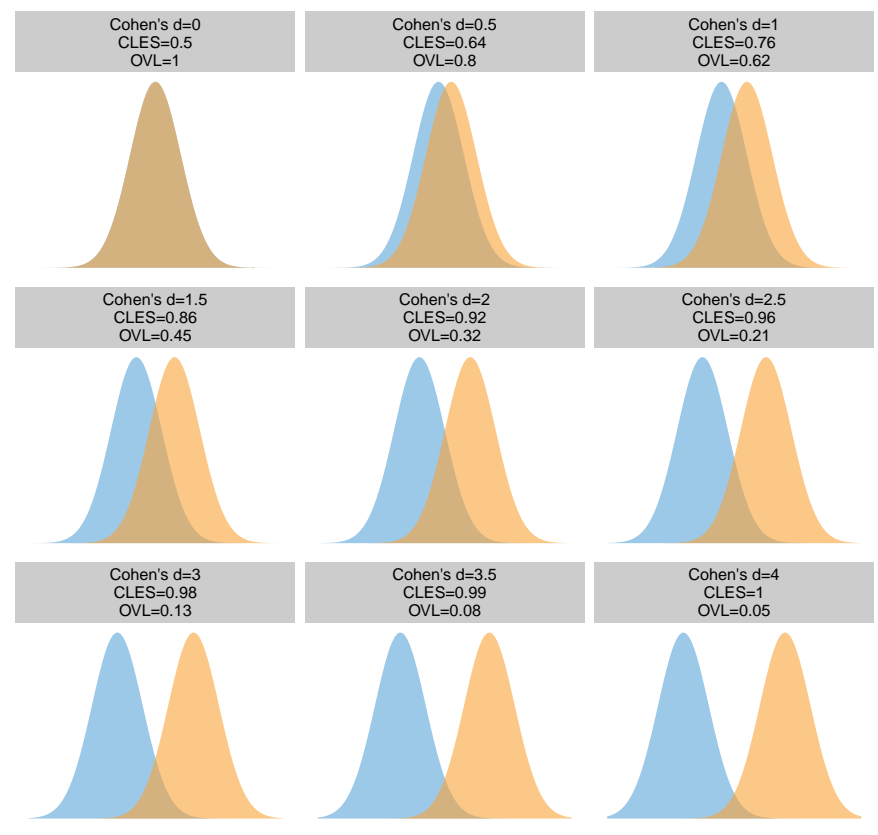

B

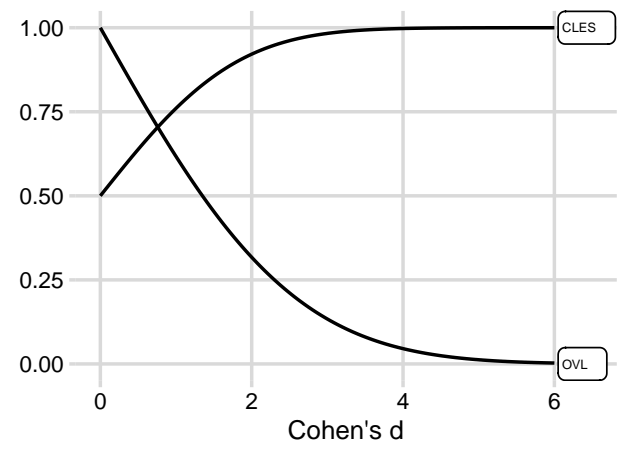

C

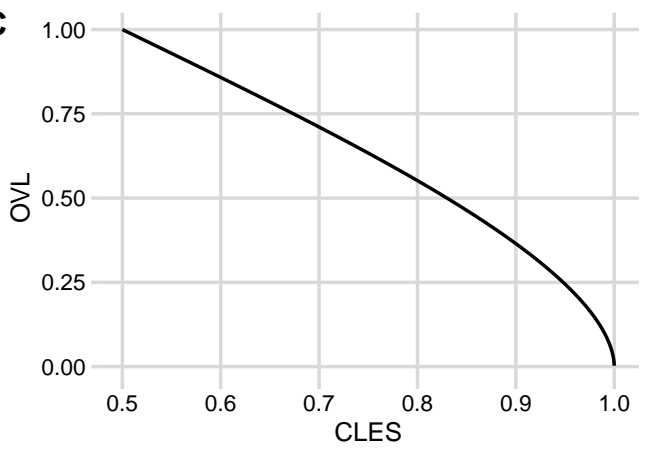

Figure 4. Relationship between the Cohen's d, CLES, and OVL. A. Visual display of the samples of varying degrees of separations, and calculated Cohen's d, CLES, and OVL. B. Relationship between the CLES and OVL to the Cohen's d. C. Relationship between the CLES and OVL

Table 5 contains Cohen's d magnitudes of effect with accompanying estimated CLES and OVL thresholds.

Table 5. Magnitudes of effect for CLES and OVL estimated using Cohen's d

\begin{tabular}{lllllll}
\hline Magnitude of effect & Trivial & Small & Moderate & Large & Very Large & Nearly Perfect \\
\hline Cohen's d & $0-0.2$ & $0.2-0.6$ & $0.6-1.2$ & $1.2-2.0$ & $2.0-4.0$ & $>4.0$ \\
CLES & $0.50-0.56$ & $0.56-0.66$ & $0.66-0.80$ & $0.80-0.92$ & $0.92-1.00$ & 1.00 \\
OVL & $1.0-0.92$ & $0.92-0.76$ & $0.76-0.55$ & $0.55-0.32$ & $0.32-0.05$ & 0.00 \\
\hline
\end{tabular}




\subsubsection{The Smallest Effect Size Of Interest}

According to Cohen (Cohen, 1988), the qualitative magnitude thresholds from Table 5 are "arbitrary conventions, recommended for use only when no better basis for estimating the effect size is available" (p. 12). But what if practitioners a priori know what is the minimal important effect size and are interested in judging the practical or clinical significance (Sainani, 2012)of the results (in this case difference between the groups)? In other words, the smallest effect size of interest (SESOI) ${ }^{8}$.

There is no single way to approach definition and estimation of SESOI, but it usually tends to be based on either the known measurement error (ME) (e.g. the minimum detectable effect size), or the effect size that is large enough to be practically meaningful (e.g. the minimal important difference, or the smallest worthwhile change) (Hopkins, 2004b, 2015b; King, 2011; Turner et al., 2015; Swinton et al., 2018; Lakens, Scheel \& Isager, 2018; Anvari \& Lakens, 2019; Caldwell \& Cheuvront, 2019). In this paper, statistical models and estimators that utilize SESOI are referred to as magnitude-based.

To introduce magnitude-based estimators, let's consider $\pm 2.5 \mathrm{~cm}$ to be body height $\mathrm{SESOI}^{9}$, or the difference that would be practically significant. In other words, individuals with height difference within $\pm 2.5 \mathrm{~cm}$ would be considered practically equivalent, or it might be hard to detect this difference with a quick glance.

The simplest magnitude-based statistics would be mean difference divided by SESOI (Difference to SESOI) (equation 8). This estimator, similar to other standardized estimators (e.g., \% CVdiff, Cohen's d) allows comparison of variables at different scales, but it would also give more insight into differences from practical significance perspective.

$$
\text { Difference to } S E S O I=\frac{\text { mean }_{\text {difference }}}{S E S O I_{\text {upper }}-S E S O I_{\text {lower }}}
$$

Second magnitude-based statistic is standard deviation of the difference divided by SESOI (SDdiff to SESOI) (equation 9). This estimator, similar to \% CVdiff, would answer how variable are the differences compared to SESOI.

$$
\text { SDdiff to SESOI }=\frac{S D_{\text {difference }}}{S E S O I_{\text {upper }}-S E S O I_{\text {lower }}}
$$

Similarly, CLES estimator can become magnitude-based by utilizing SESOI. Rather than being interested in probability of a random male being taller than a random female (out of the two sample groups), we might be interested in estimating how probable are lower, trivial, and higher (or usually defined as harmful, trivial, and beneficial) differences defined by SESOI. Trivial differences are differences ranging from $S E S O I_{\text {lower }}$ to $S E S O I_{\text {upper }}$, while everything over $S E S O I_{\text {upper }}$ is higher (or beneficial) difference and everything lower than $S E S O I_{\text {lower }}$ is lower (or harmful) difference.

Using brute-force computational method and drawing all pair-wise combinations from the two groups (50x50 $=2500$ cases), and using $\pm 2.5 \mathrm{~cm}$ SESOI as a trivial difference ${ }^{10}$, we can estimate probabilities of lower, trivial and higher difference by calculating proportion of cases within each magnitude band (Figure 5).

\footnotetext{
${ }^{8}$ Other name used is region of practical equivalence (ROPE) (Kruschke \& Liddell, 2018a,b)

${ }^{9}$ SESOI has two thresholds: lower and upper, or negative and positive. In this example these thresholds are $-2.5 \mathrm{~cm}$ and $+2.5 \mathrm{~cm}$. This makes SESOI range equal to $5 \mathrm{~cm}$, which is calculated as $S E S O I_{\text {upper }}-S E S O I_{\text {lower }}$. This range can also be refered to as equivalence range.

${ }^{10}$ It is here assumed here that SESOI is symmetrical in both positive and negative directions. This makes the trivial difference ranging from $-2.5 \mathrm{~cm}$ to $+2.5 \mathrm{~cm}$. SESOI doesn't necessary needs to be symmetrical in both lower and upper thresholds.
} 

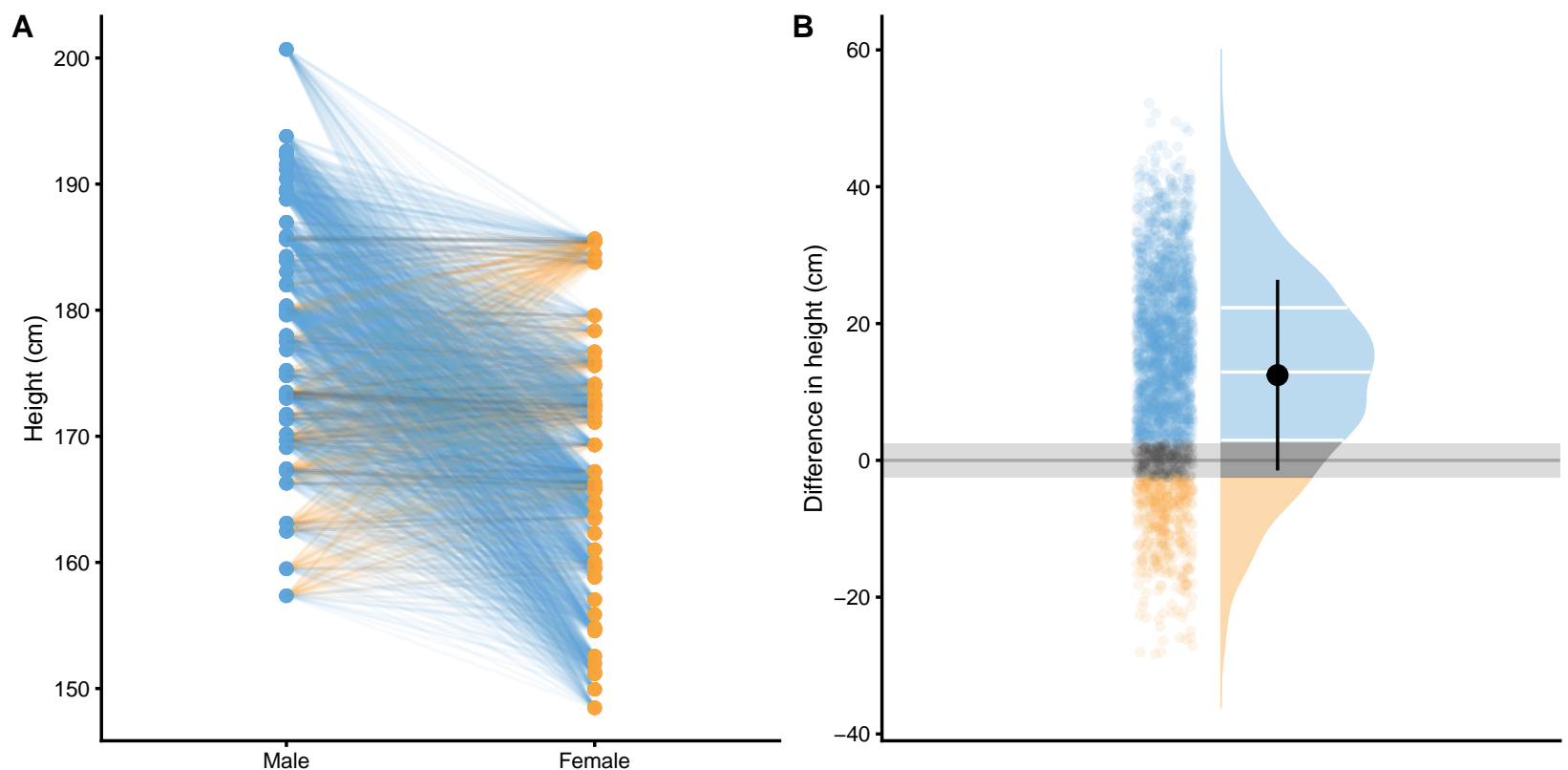

Figure 5. Pairwise comparison of males and females to estimate probability of lower, trivial, and higher magnitude of effect. A. Scatterplot of all pair-wise combinations $(50 x 50=2500)$, drawn at random out of two samples. Since we are comparing paired males and females, lines can be drawn between each of 2500 draws. Blue line indicates males taller than females higher than SESOI, trivial lines indicates pairs with a height difference less or equal to SESOI, while orange line indicates females taller than males higher than SESOI. B. Distribution of the differences between males and females for all 2500 pair-wise combinations. Grey band indicates SESOI. Surface of the distribution over SESOI (blue color) indicates probability of randomly selected male being taller than a randomly selected female, with a height difference of at least SESOI magnitude. Surface of the distribution under SESOI (orange color) indicates probability of randomly selected female being taller than a randomly selected female, with a height difference of at least SESOI magnitude. Grey surface area indicates probability of randomly selecting male and female with a height difference within SESOI band

Table 6 contains estimated probabilities of observing lower, trivial, and higher differences in height between the randomly selected male and female using brute-force computational method and algebraic method. These estimates answer the following question "If I compare random male and random female from my sample, how probable are lower/trivial/higher magnitudes of difference in height?". Asking such a magnitude-based question regarding the random individual difference represents a form of prediction question and predictive task. In this paper, such questions are answered with magnitude-based prediction approaches.

Table 6. Estimated probabilities of observing lower, trivial, and higher differences in height

\begin{tabular}{lrrr}
\hline Method & lower & trivial & higher \\
\hline brute-force & 0.15 & 0.09 & 0.76 \\
algebraic & 0.14 & 0.10 & 0.76 \\
\hline
\end{tabular}

It is common to represent means as systematic component or fixed effect (e.g. average causal effect), and variability around the mean (i.e. SD) as stochastic component or random effect. It is unfortunate that the common statistical modelling and analysis, particularly in sport science, takes the stance of approaching and treating between-individual variation as random error. The approach suggested in this paper complements group-based or average-based statistics with magnitude-based predictions that aim to help in answering individual-based questions, common to sport practitioners. Table 7 contains discussed magnitude-based 
estimators that can complement common effect size statistics (Table 2) when comparing two independent groups.

Table 7. Magnitude-based effect size statistics for estimating difference between two independent groups

\begin{tabular}{rrrrrrr}
\hline SESOI lower $(\mathrm{cm})$ & SESOI upper $(\mathrm{cm})$ & Difference to SESOI & SDdiff to SESOI & Lower & Trivial & Higher \\
\hline-2.5 & 2.5 & 2.49 & 2.82 & 0.14 & 0.1 & 0.76 \\
\hline
\end{tabular}

\subsubsection{Comparing dependent groups}

As an example of dependent or paired groups descriptive analysis, let's consider the simple pre-test (pre) and post-test (post) design. We have given training intervention to a group of $\mathrm{N}=20$ males involving benchpress training. Training intervention involved performing bench pressing two times a week for 16 weeks. One-repetition-maximum (1RM) in the bench press was performed before (Pre) and after (Post) training intervention. Table 8 contains individual Pre and Post scores, as well as the Change in the bench press 1RM.

Table 8. Individual Pre and Post scores, as well as Change in the bench press 1RM

\begin{tabular}{rrrr}
\hline athlete & Pre $(\mathrm{kg})$ & Post $(\mathrm{kg})$ & Change $(\mathrm{kg})$ \\
\hline 1 & 92.84 & 122.57 & 29.73 \\
2 & 103.10 & 84.87 & -18.23 \\
3 & 108.00 & 116.73 & 8.73 \\
4 & 101.37 & 106.26 & 4.89 \\
5 & 89.95 & 90.50 & 0.54 \\
6 & 96.18 & 90.13 & -6.05 \\
7 & 90.43 & 83.76 & -6.67 \\
8 & 77.21 & 91.69 & 14.47 \\
9 & 108.34 & 125.07 & 16.73 \\
10 & 101.68 & 111.58 & 9.90 \\
11 & 115.44 & 117.39 & 1.95 \\
12 & 94.03 & 99.57 & 5.53 \\
13 & 109.60 & 117.61 & 8.01 \\
14 & 100.62 & 123.17 & 22.55 \\
15 & 108.20 & 120.58 & 12.38 \\
16 & 105.07 & 107.09 & 2.02 \\
17 & 89.08 & 111.77 & 22.69 \\
18 & 86.40 & 69.93 & -16.47 \\
19 & 94.97 & 96.42 & 1.45 \\
20 & 97.80 & 102.24 & 4.43 \\
\hline
\end{tabular}

The results of this simple Pre and Post design can be described in multiple ways. Here, I will present the three most common approaches. 


\subsubsection{Describing groups as independent}

The simplest analysis involve descriptive statistics assuming groups as independent. Table 9 contains descriptive statistics applied to Pre, Post and Change scores as independent. Figure 6 visualizes the scores using three raincloud plots.

Table 9. Descriptive analysis of Pre-test, Post-test and Change as independent samples

\begin{tabular}{|c|c|c|c|c|c|c|c|c|c|c|c|c|c|}
\hline Test & $\mathrm{n}$ & mean $(\mathrm{kg})$ & $\mathrm{SD}(\mathrm{kg})$ & $\% \mathrm{CV}$ & median $(\mathrm{kg})$ & MAD (kg) & IQR $(\mathrm{kg})$ & mode $(\mathrm{kg})$ & $\min (\mathrm{kg})$ & $\max (\mathrm{kg})$ & range $(\mathrm{kg})$ & skew & kurtosis \\
\hline Pre & 20 & 98.52 & 9.39 & 10 & 99.21 & 11.23 & 13.56 & 99.11 & 77.21 & 115.44 & 38.23 & -0.27 & -0.60 \\
\hline Post & 20 & 104.45 & 15.61 & 15 & 106.68 & 18.41 & 26.05 & 115.80 & 69.93 & 125.07 & 55.14 & -0.44 & -0.96 \\
\hline Change & 20 & 5.93 & 12.26 & 207 & 5.21 & 8.79 & 11.68 & 5.26 & -18.23 & 29.73 & 47.95 & -0.12 & -0.48 \\
\hline
\end{tabular}

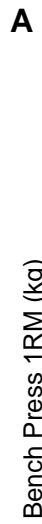

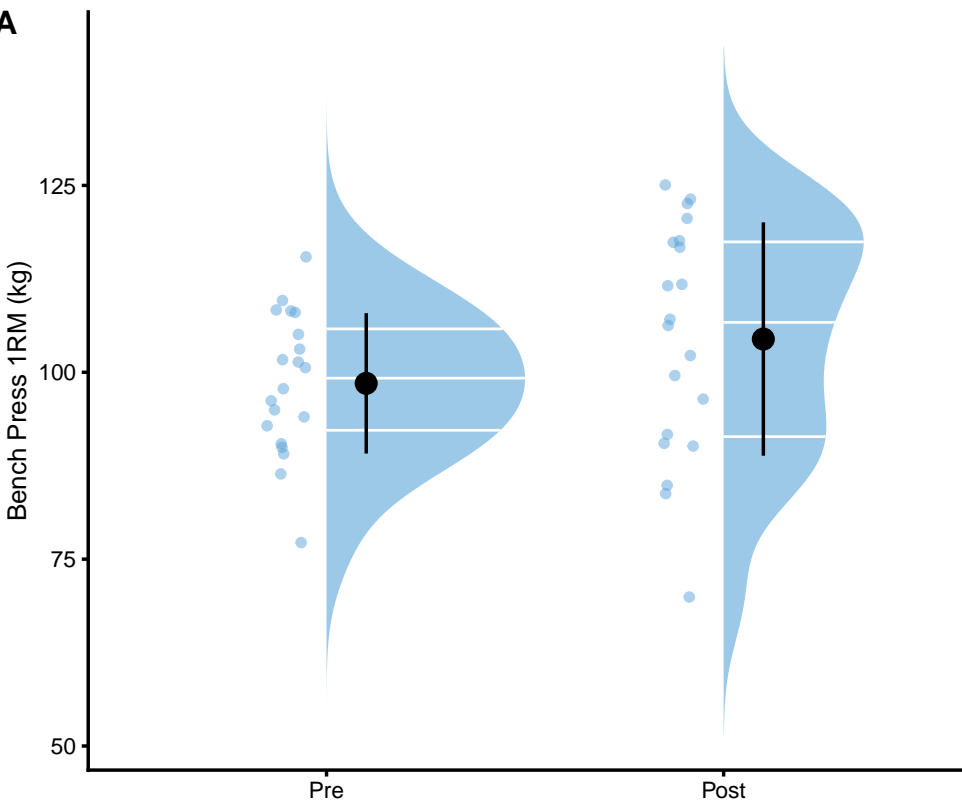

B

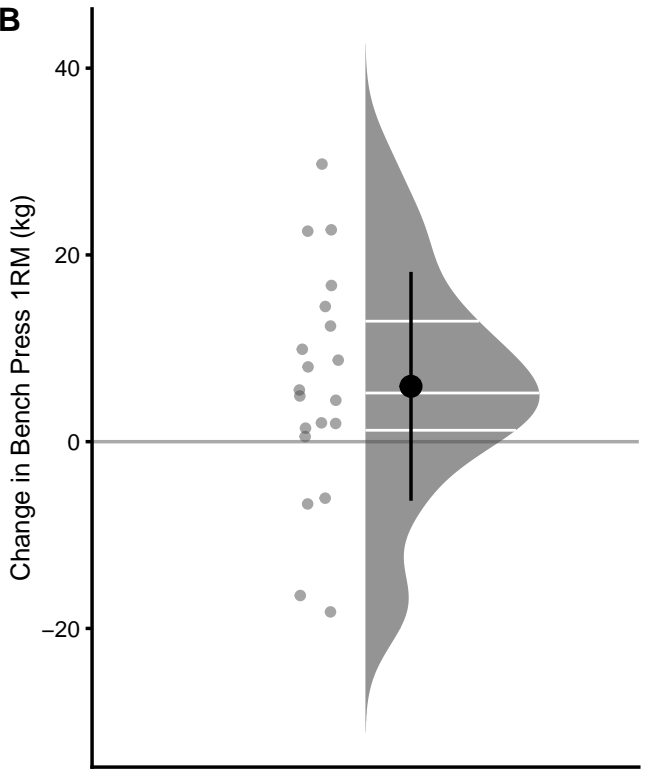

Figure 6. Raincloud plots of the Pre-test, Post-test and Change scores in the bench press 1RM. A. Distribution of the Pre-test and Post-test scores. B. Distribution of the Change score

\subsubsection{Effect Sizes}

Table 10 contains the most common effect sizes utilized when describing change in the Pre-Post paired design. The terminology utilized in this paper differentiate between the difference which is used in independent groups and the change which is used in paired or dependent groups.

Table 10. Effect size statistics for estimating change in two dependent groups

\begin{tabular}{rrrrrrrr}
\hline Change $(\mathrm{kg})$ & SDchange $(\mathrm{kg})$ & \% CVchange & \% Change & Ratio & Cohen's d & CLES & OVL \\
\hline 5.93 & 12.26 & 207 & 6 & 1.06 & 0.63 & 0.69 & 0.82 \\
\hline
\end{tabular}


Change, or mean change is calculated by taking average of the change score (equation 10). Change score is simple difference between Pre and Post:

$$
\begin{aligned}
\text { mean }_{\text {change }} & =\frac{1}{n} \sum_{i=1}^{n}\left(\text { post }_{i}-\text { pre }_{i}\right) \\
\text { mean }_{\text {change }} & =\frac{1}{n} \Sigma_{i=1}^{n} \text { change }_{i} \\
\text { change }_{i} & =\text { post }_{i}-\text { pre }_{i}
\end{aligned}
$$

SDchange, or standard deviation of the change is a simple standard deviation of the change (equation 11). It represents a measure of dispersion of the change scores:

$$
S D_{\text {change }}=\sqrt{\frac{1}{n-1} \Sigma_{i=1}^{n}\left(\text { change }_{i}-\text { mean }_{\text {change }}\right)^{2}}
$$

$\% C V$ change, or percent coefficient of variation of the change is the standard deviation of the change divided by mean change (equation 12):

$$
\% C V_{\text {change }}=100 \times \frac{S D_{\text {change }}}{\text { mean }_{\text {change }}}
$$

\% Change, or Mean percent change is calculated by taking a mean of the ratio between the change and the pre-test, multiplied by 100 (equation 13):

$$
\text { mean }_{\% \text { change }}=100 \times \frac{1}{n} \Sigma_{i}^{n} \frac{\text { change }_{i}}{\text { pre }_{i}}
$$

Mean ratio represents mean of the post to pre ratios (equation 14):

$$
\text { mean }_{\text {ratio }}=\frac{1}{n} \Sigma_{i}^{n} \frac{\text { post }_{i}}{\text { pre }_{i}}
$$

Cohen's $d$ represents standardized effect size for the change. In the paired design, Cohen's d is calculated by dividing mean change with standard deviation of the pre-test scores (equation 15):

$$
\text { Cohen's } d=\frac{\text { mean }_{\text {change }}}{S D_{\text {pre }}}
$$

$C L E S$ for the paired groups represents probability of observing positive change. OVL, equally to the independent groups, represents overlap between the pre and post scores.

Magnitude-based effect size estimators involve the use of SESOI and can be found on Table 11. Similarly to magnitude-based effect size estimators with the independent groups, magnitude-based effect size estimators with the paired group involve Change to SESOI, SDchange to SESOI as well as proportions of lower, trivial and higher change scores.

Table 11. Magnitude-based effect size statistics for estimating change between two dependent groups

\begin{tabular}{lrrrrr}
\hline SESOI $(\mathrm{kg})$ & Change to SESOI & SDchange to SESOI & Lower & Trivial & Higher \\
\hline \pm 5 & 0.59 & 1.23 & 0.19 & 0.28 & 0.53 \\
\hline
\end{tabular}


Figure 7 depicts visually how proportions of lower, trivial and higher change scores are estimated. Same as with two independent groups, these proportions can be estimated using the brute-force method (i.e. simplecounting of the change scores withing lower, trivial, and higher zones), or algebraic where SDchange is utilized and assumption of the normally distributed change scores is made.
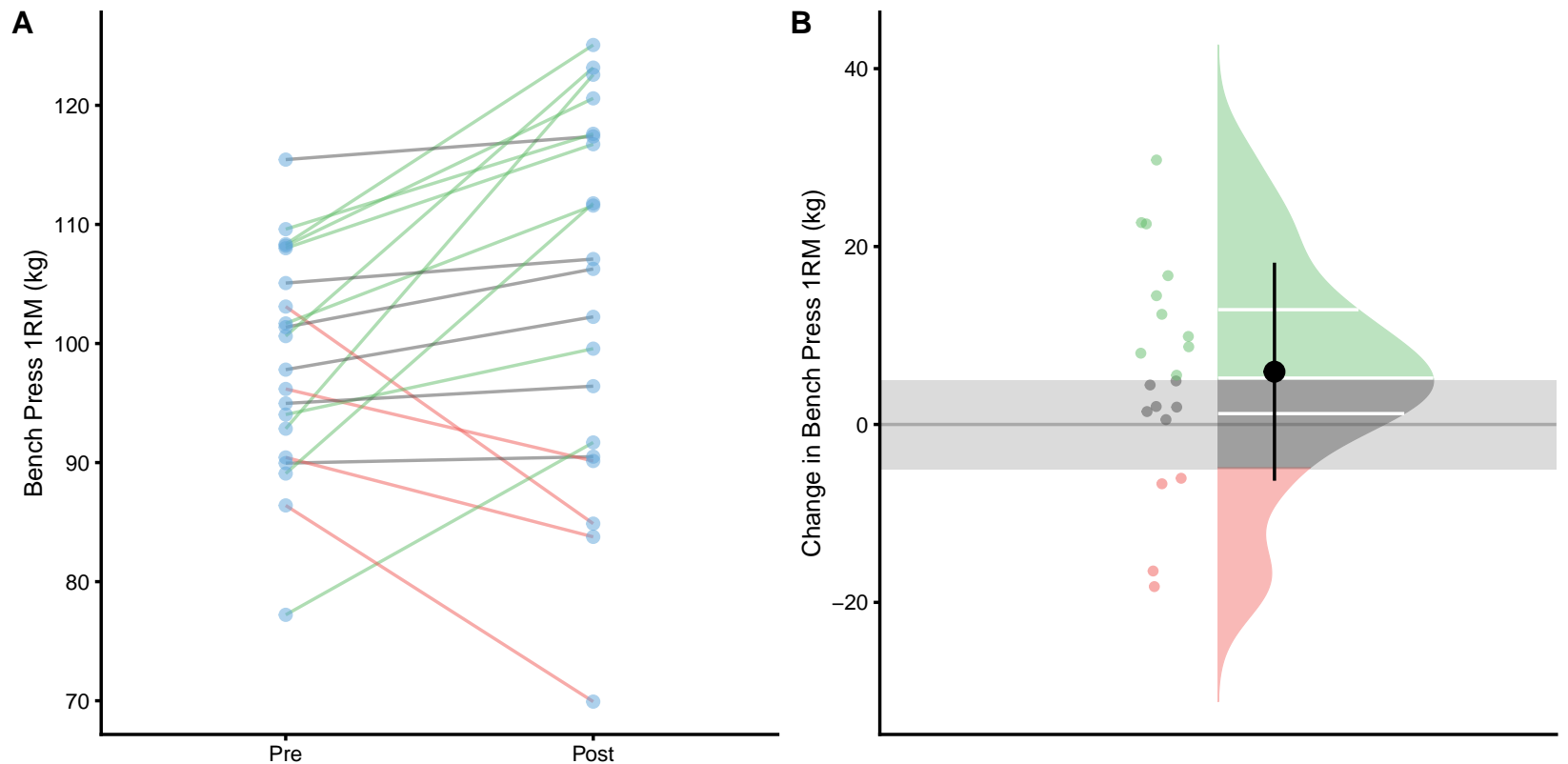

Figure 7. Visual analysis of the dependent groups scores using SESOI. A. Scatter plot of Pre-test and Post-test scores. Green line indicates change higher than SESOI upper, grey line indicates change within SESOI band, and red line indicates negative change lower than SESOI lower. B. Distribution of the change scores. Green area represents proportion of change scores higher than SESOI upper, red area represents proportion of negative change scores lower than SESOI lower, and grey area indicates trivial change, which is within SESOI band

It might be tempting to claim that this intervention is causing changes in the bench press 1RM, but we should be vary of doing that. It is important to keep in mind that the effect size estimators are used only descriptively without any causal connotation. To make causal claims, further criteria needs to be taken into account. This is discussed in more details in the "Causal Inference" section of this paper.

\subsubsection{Describing relationship between two variables}

So far, we have dealt with single variable descriptive statistics. However, we are often interested in relationship or association between two variables. One of these variables takes the role of the dependent variable (outcome or target variable) and the other of the independent variable (or predictor variable).

Let's assume we tested $\mathrm{N}=30$ female soccer athletes by using two tests: (1) Yo-YoIR1, and (2) maximum aerobic speed (MAS) test. Variables in this example represent observations in each test. Visual analysis in Figure 8 depicts the association between these two tests using scatter plot. 


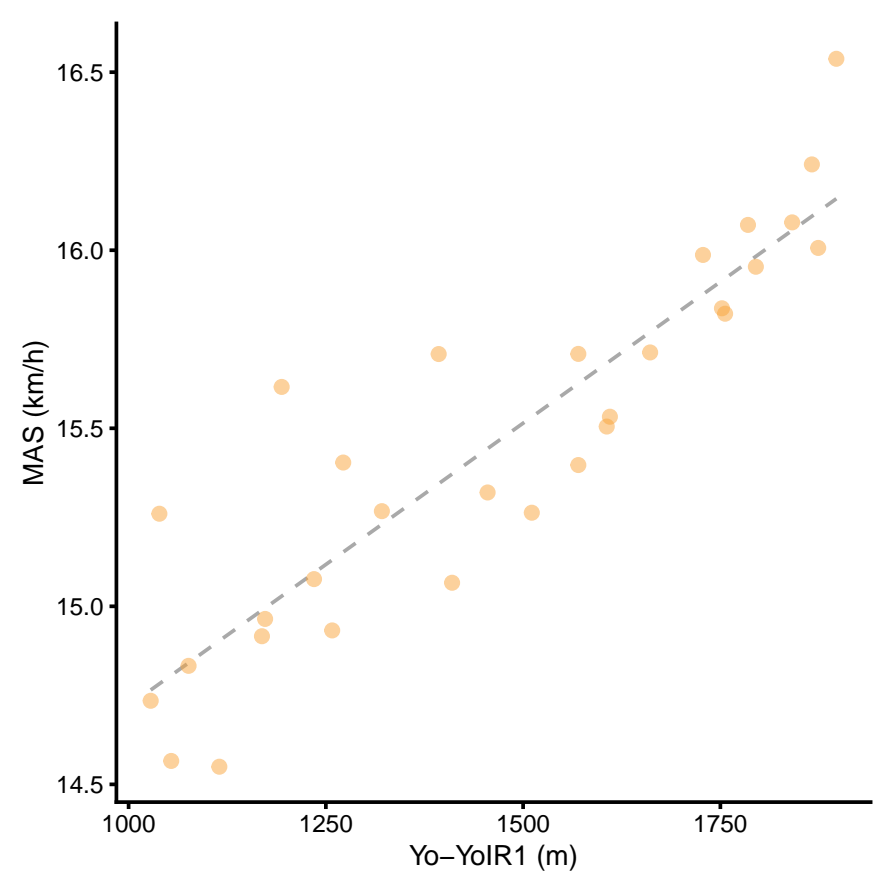

Figure 8. Scatter plot between two variables. Dashed line represents linear regression line.

Table 12 contains common estimators of the association between two variables. All estimators except maximum information coefficient (MIC) (Reshef et al., 2011; Albanese et al., 2012) assumes linear relationship between two variables. It is thus important to visually analyze the association (see Figure 9) before trusting numerical estimators.

Table 12. Common estimators of the association between two variables

\begin{tabular}{rrr}
\hline Pearson $\mathrm{r}$ & R-squared & MIC \\
\hline 0.89 & 0.8 & 0.92 \\
\hline
\end{tabular}

The Pearson product-moment correlation coefficient (Pearson's $\mathrm{r}$ ) is a measure of the strength of the linear relationship between two variables (equation 16):

$$
r=\frac{\sum_{i=1}^{n}\left(x_{i}-\bar{x}\right)\left(y_{i}-\bar{y}\right)}{\sqrt{\sum_{i=1}^{n}\left(x_{i}-\bar{x}\right)^{2}\left(y_{i}-\bar{y}\right)^{2}}}
$$

Pearson's $\mathrm{r}$ is standardized measure that can take values ranging from -1 to +1 , where 0 indicates no relationship, and -1 and +1 indicates perfect relationship. Negative Pearson's $r$ value represents negative association (i.e. as one variable increases the other decreases), while positive Pearson's $r$ value represents positive association (i.e., as one variable increases so does the other).

$R$-squared $\left(R^{2}\right)$ represents variance explained, i.e. how much the model explains variance in the target variable. In this example the model is linear regression. R-squared is standardized measure of association that can take values ranging from zero (no association, or no variance explained) to 1 (perfect association, or all association explained). R-squared, as its name suggests, represents Pearson's r squared, but for more complex models it can be calculated using variances or mean squares (MS) (equation 17): 


$$
\begin{aligned}
R^{2} & =\frac{M S_{\text {model }}}{M S_{\text {total }}} \\
M S_{\text {model }} & =\frac{1}{n} \sum_{i=1}^{n}\left(\hat{y}_{i}-\bar{y}\right)^{2} \\
M S_{\text {total }} & =\frac{1}{n} \sum_{i=1}^{n}\left(y_{i}-\bar{y}\right)^{2}
\end{aligned}
$$

Maximal information coefficient (MIC) is a novel measure of the strength of the linear or non-linear association between two variables and belongs to the maximal information-based non-parametric exploration (MINE) class of statistics (Reshef et al., 2011; Albanese et al., 2012). MIC is standardized measure of association that can take values ranging from zero (no association) to 1 (perfect association). As opposed to Pearson r, MIC can pick up non-linear association between two variables. But as will be demonstrated in the "Prediction" section of this paper, both Pearson's $r$ and R-squared can be utilized with models more complex than simple linear regression.

Statistical model, or machinery underlying Pearson $\mathrm{r}$ and R-squared is linear regression. Similar to a sample mean, linear regression can be seen as optimization algorithm that tries to find a line that passes through the data with the minimal error. ${ }^{11}$ A solution to this problem can be found computationally or analytically. Either way, the parameters that need to be estimated in this example with two variables are intercept $\left(\hat{\beta}_{0}\right)$, slope coefficient $\left(\hat{\beta}_{1}\right)$, and error or residual $(\hat{\epsilon})$ (equation 18$)$ :

$$
\hat{y}_{i}=\hat{\beta}_{0}+\hat{\beta}_{1} x_{i}+\hat{\epsilon}
$$

Table 13 contains estimates for intercept, slope, and error. Error $(\epsilon)$ is estimated by using residual standard error (RSE), which is similar to already discussed RMSE, but rather than dividing sum of square errors by $n$ observations, it is divided by $n-p$ (equation 19). The $p$ is the number of model parameters, in this case 2 (intercept and one slope coefficient).

$$
R S E=\sqrt{\frac{1}{n-p} \sum_{i=1}^{n}\left(y_{i}-\hat{y}_{i}\right)^{2}}
$$

Table 13. Linear regression estimates for intercept, slope, and error

\begin{tabular}{rrr}
\hline Intercept $(\mathrm{km} / \mathrm{h})$ & Slope & $\mathrm{RSE}(\mathrm{km} / \mathrm{h})$ \\
\hline 13.13 & 0.00159 & 0.23 \\
\hline
\end{tabular}

The linear equation (20) describing the relationship between the Yo-YoIR1 and MAS is as follows:

$$
\text { MAS }=13.13+0.00159 \times \text { Yo-YoIR1 }
$$

Slope coefficient of 0.00159 can be interpreted the following way: if Yo-YoIR1 increases by 500m, then MAS would increase by $500 \times 0.00159$ or $0.79 \mathrm{~km} / \mathrm{h}$.

Although measures of association between two variables, such as Pearson's $r$ and $R$-squared, are symmetrical (meaning it doesn't matter which variable is predictor or target), one cannot reverse the linear regression equation to get Yo-YoIR1 from MAS. Equation (21) shows how to reverse the parameters to estimate Yo-YoIR1 from known MAS:

\footnotetext{
${ }^{11}$ This approach, as already explained, belongs to the OLS approach. On the other hand, MLE tries to find a line that maximizes likelihood of the data.
} 


$$
\begin{aligned}
M A S & =\hat{\beta}_{0}+\hat{\beta}_{1} Y_{o}-Y o I R 1 \\
Y o-Y o I R 1 & =\frac{M A S-\hat{\beta}_{0}}{\hat{\beta}_{1}} \\
Y_{O}-Y_{O I R} 1 & =\frac{1}{\hat{\beta}_{1}} M A S-\frac{\hat{\beta}_{0}}{\hat{\beta}_{1}}
\end{aligned}
$$

As shown in the Table 14 and Figure 9, the reversal done by Equation 18 shouldn't be done, since for linear regression it matters which variable is target and which one is predictor. If you calculate the reversal parameters using Equation 18, you will notice that they are not equal to those in the Table 14. This is also indicated with non-identical linear regression lines in the Figure 9.

Table 14. Reverse estimates

\begin{tabular}{rrr}
\hline Intercept $(\mathrm{m})$ & Slope & RSE $(\mathrm{m})$ \\
\hline-6330.83 & 504.32 & 131.55 \\
\hline
\end{tabular}

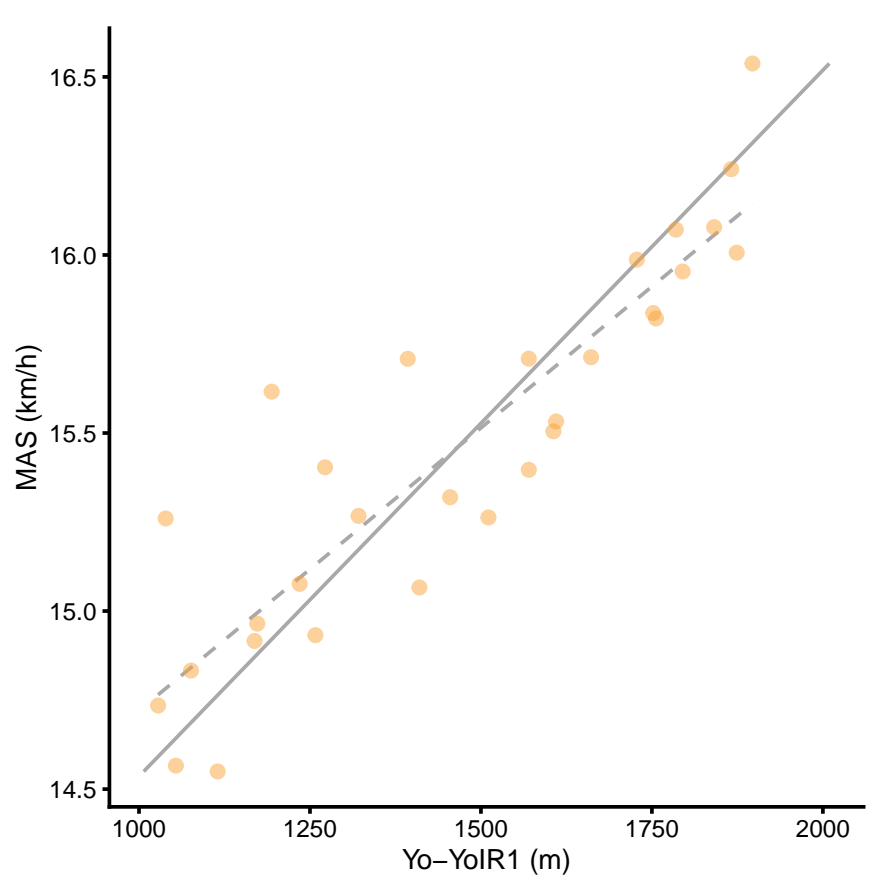

Figure 9. Regression line differs depending which variable is target or the outcome variable. Dashed grey line represents regression line when MAS is the target variable. Grey line represents regression line when Yo-YoIR1 is the target variable. Since they are not identical, one cannot reverse the equation to predict Yo-YoIR1 from MAS score, when such equation is estimated by predicting MAS from Yo-YoIR1

\subsubsection{Magnitude-based estimators}

Similarly to independent and dependent group, with association we might be interested in the practical significance of the results. In order to judge results from practical significance perspective, we need to define SESOI of both variables (i.e. Yo-YoIR1 and MAS). For example, we might be interested if minimal important 
change in the Yo-YoIR1 is associated with minimal important change in the MAS. Let's call this association sensitivity estimator. This can be estimated with the following equation (22):

$$
\text { Sensitivity }=\frac{\left(S E S O I_{Y o-Y o I R 1_{\text {upper }}}-S E S O I_{Y o-Y o I R 1_{\text {lower }}}\right) \times \hat{\beta}_{1}}{S E S O I_{M A S_{\text {upper }}}-S E S O I_{M A S_{\text {lower }}}}
$$

Assuming SESOI in the Yo-YoIR1 is $\pm 500 \mathrm{~m}$, SESOI in the MAS is $\pm 0.25 \mathrm{~km} / \mathrm{h}$, and by using already estimated $\hat{\beta}_{1}$, we can calculate the sensitivity: 3.18 . This means that minimal important change in the Yo-YoIR1 will be associated with much bigger minimal important change in the MAS. If either SESOI of the MAS or Yo-YOIR1 are unknown, using SESOI of one variable can be helpful to estimate SESOI of the other variable by using the slope coefficient $\hat{\beta}_{1}$.

Next question might be related to the practically significant strength of the association between two variables. For example, we would like to know if the residuals are higher or lower than the SESOI in the target variable (i.e. MAS). Figure 10 depicts scatter plot between two variable (panel A) and residuals (panel B) utilizing SESOI in MAS as the grey area.
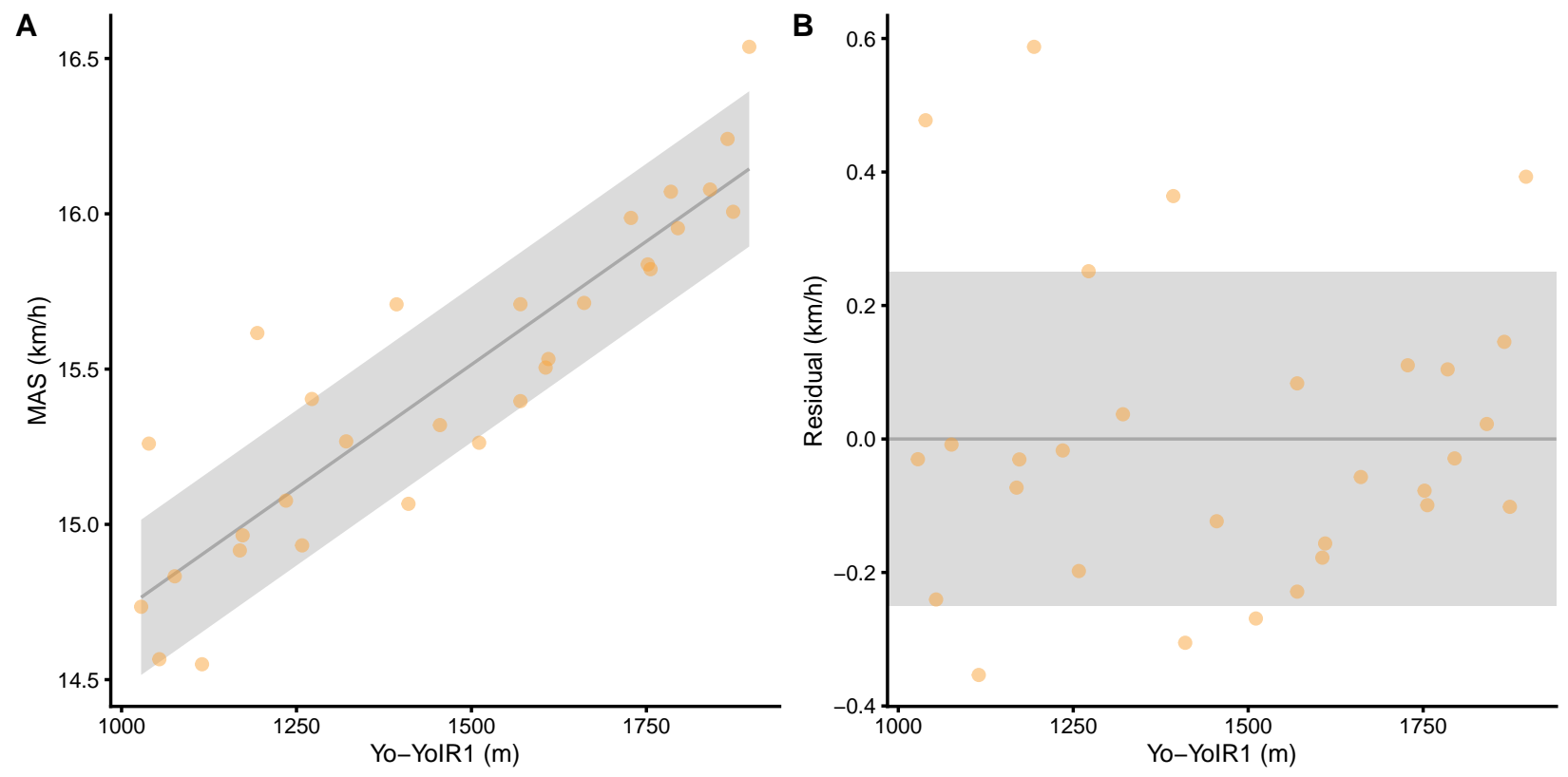

Figure 10. Scatter plot between two variables using SESOI to indicate practically significant difference A. Scatterplot with SESOI depicted as grey band around linear regression line. B. Residual plot, where the difference between MAS and linear regression line (model estimate) is plotted against Yo-YoIR1 variable. SESOI is represented with the grey band. Residuals within SESOI band are of no practical difference

Magnitude-based estimators of the practically significant strength of the association involve ratio between the SESOI (SESOI $I_{\text {upper }}-S E S O I_{\text {lower }}$ ) and RSE, and Equivalence. Ratio between SESOI and RSE (SESOI to $R S E$ ) indicates how big are the residuals compared to the SESOI, and thus a metric of the quality of the association. Assuming that residuals are being normally distributed, SESOI to RSE over 4 (or $2 \times 1.96$ ) would indicate excellent practical strength of the association. If you look at the Table 15, estimated SESOI to RSE in this example is not great, indicating poor practical strength of association.

Equivalence as a measure of the practical strength of the association revolves around estimating proportions of residuals in the trivial zone, defined as SESOI in the target variable. Similar to lower, trivial and higher difference and changes, equivalence can be estimated with the brute-force method by simply counting residuals 
in the trivial zone, or using algebraic method and assuming normally distributed residuals (i.e. using SD of the residuals). Equivalence can be computed algebraically by utilizing RSE instead of residual SD, but in order to make it comparable with predictive models from "Prediction" section of this paper, residual SD is used instead.

Figure 11 graphically depicts how equivalence estimator is calculated. Practically significant association between two variables would have equivalence equal to 1 , which indicates that all residuals are within confines of the SESOI. If you look at the Table 15, estimated equivalence in this example is less than perfect, indicating poor practical strength of the association.

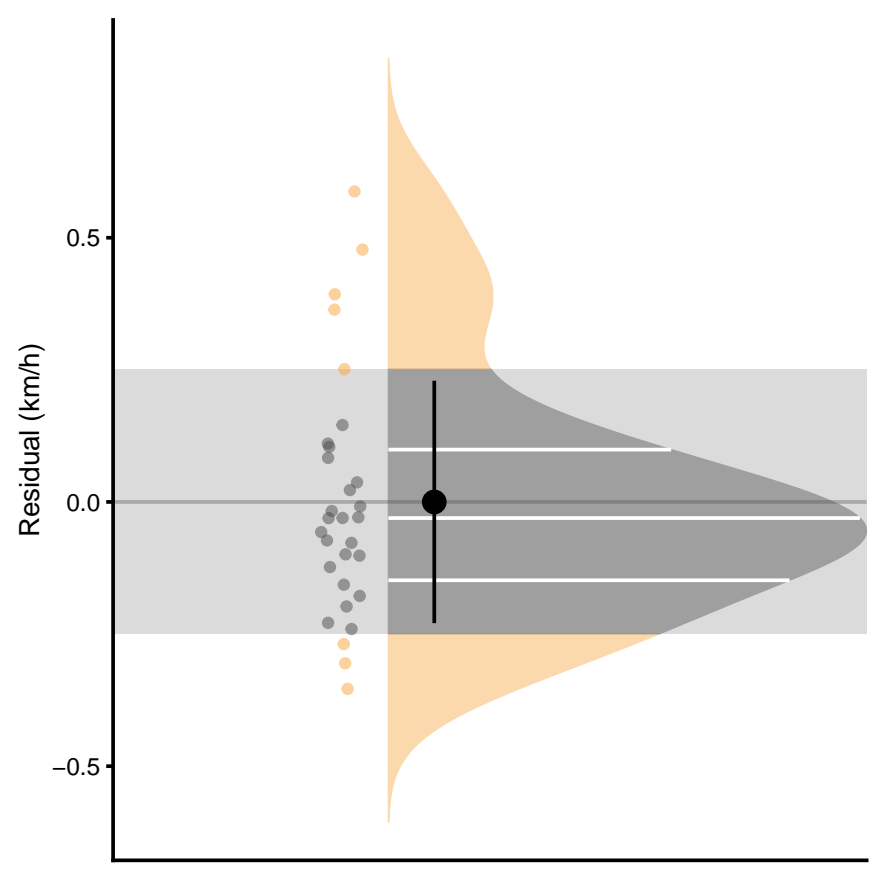

Figure 11. Equivalence is proportion of residuals within SESOI band

Table 15. Magnitude-based estimators of the association between two variables

\begin{tabular}{llrrrr}
\hline SESOI Yo-YoIR1 $(\mathrm{m})$ & SESOI MAS $(\mathrm{km} / \mathrm{h})$ & Sensitivity & RSE & SESOI MAS to RSE & Equivalence \\
\hline \pm-500 & \pm 0.25 & 3.18 & 0.23 & 2.14 & 0.72 \\
\hline
\end{tabular}

SESOI to RSE (or SESOI to RMSE) and Equivalence will be utilized in predictive tasks (see "Prediction" section of this article) to estimate magnitude-based performance of the predictive models.

\subsubsection{Advanced uses}

Advanced techniques in the descriptive statistics involve dimension reduction, such as principal component analysis (PCA), latent variable modelling, such as factor analysis (FA), or cluster analysis (Borsboom, Mellenbergh \& van Heerden, 2003; Borsboom, 2008; Everitt \& Hothorn, 2011; Beaujean, 2014; Finch \& French, 2015; Kabacoff, 2015). These techniques are beyond the scope of this paper and the interested readers are directed to references provided. 


\subsection{Prediction}

In many disciplines there is a near-exclusive use of the statistical models for causal inference ${ }^{12}$ and the assumption that models with high explanatory power are inherently of high predictive power (Breiman, 2001; Shmueli, 2010; Yarkoni \& Westfall, 2017; Hernán, Hsu \& Healy, 2019). There is a constant tug-of-war between prediction versus explanation, and experts are leaning on one side or the other. Some experts warn against over-reliance on explanatory models with poor predictive power (Breiman, 2001; Shmueli, 2010; Yarkoni \& Westfall, 2017), whereas some warn against over-reliance on predictive models that lack causal explanatory power that can guide intervention (Pearl, Glymour \& Jewell, 2016; Pearl \& Mackenzie, 2018; Hernán, Hsu \& Healy, 2019; Pearl, 2019).

It is thus important to differentiate between the two and take into account the research question that we are trying to answer. In this paper, I define predictive modeling by using definition from Galit Shmueli (Shmueli, 2010) "as the process of applying a statistical model or data mining algorithm to data for the purpose of predicting new or future observations" (p. 291). Usually this predictive statistical model is treated as a black box. Black box approach implies that we are not really interested in underlying mechanism and relationships between the predictor variables, only in predictive performance of the model (Breiman, 2001; Shmueli, 2010; Yarkoni \& Westfall, 2017)

Linear regression model from "Describing relationship between two variables" section of this paper can be used to help answer the predictive question ("If I know someone's Yo-YoIR1 score, what would be his or her MAS score?"), rather than association one ("How is Yo-YoIR1 associated with MAS?"). But there are a few caveats with such a prediction.

\subsubsection{Overfitting}

To explain a few caveats with predictive modeling, let's take slightly more complex example. Imagine we know the true relationship between back squat relative 1RM (BS) ${ }^{13}$ and vertical jump height during a bodyweight squat jump (SJ; measured in $\mathrm{cm}$ ). This true relationship is usually referred to as data generating process (DGP) (Carsey \& Harden, 2013) and one of the aims of causal inference tasks is to uncover parameters and mechanism of DGP from the acquired sample ${ }^{14}$. With predictive tasks this aim is of no direct interest, but rather reliable prediction regarding new or unseen observations.

DGP is usually unknown, but with simulations, such as this one, DGP is known and it is used to generate the sample data. Simulation is thus excellent teaching tool, since one can play with the problem and understand how the statistical analysis works, since the true DGP is known and can be compared with estimates (Hopkins, 2007; Carsey \& Harden, 2013; Rousselet, Pernet \& Wilcox, 2019a).

DGP is assumed to consist of systematic component $f(x)$ and stochastic component $\epsilon$ (equation 23).

$$
Y=f(X)+\epsilon
$$

Systematic component is assumed to be fixed in the population (constant from sample to sample) and captures the true relationship $f(X)$ among variables in the population (e.g. this can also be termed signal), while stochastic component represents random noise or random error, that varies from sample to sample, althought

\footnotetext{
${ }^{12}$ Some authors refer to causal inference as "explanatory" modelling (Breiman, 2001; Shmueli, 2010; Yarkoni \& Westfall, 2017), although Miguel Hernan (Hernán, Hsu \& Healy, 2019) warns against using such a somewhat-misleading term "because causal effects may be quantified while remaining unexplained (randomized trials identify causal effects even if the causal mechanisms that explain them are unknown)" (p. 43). Gelman also makes distinctions between forward causal inference and reverse causal inference that might be useful in distinguishing between identifying causal effects and explaining them (Gelman, 2011). This is elaborated in the "Causal inference" section of this paper.

${ }^{13}$ For example, if an athlete lifted $175 \mathrm{~kg}$ for a single rep in the back squat, and was unable to lift more, this represents his back squat 1RM, or one repetition maximum. Relative 1RM is calculated by dividing 1RM with athlete's bodyweight. For example, an athlete with $175 \mathrm{~kg} 1 \mathrm{RM}$ weights $85 \mathrm{~kg}$. His relative $1 \mathrm{RM}$ is equal to 2.05 .

${ }^{14}$ Uncovering DGP parameters is not solely the goal of the causal inference (although causal inference task is to uncover or quantify causal mechanism), but also the main goal in the statistical inference where the aim is to quantify uncertainty about the true population parameters from the acquired sample. More about this topic in the "Statistical Inference" section.
} 
its distribution remains the same. Random error is assumed to be normally distributed with mean of 0 and standard deviation which represents estimated parameter (either with RMSE or RSE). Thus, RMSE or RSE are estimates of $\epsilon$.

In our example, the relationship between SJ and BS is expressed with the following equation (24):

$$
\begin{aligned}
S J & =30+15 \times B S \times \sin (B S)+\epsilon \\
\epsilon & \sim \mathcal{N}(0,2)
\end{aligned}
$$

Systematic component in the DGP is represented with $30+15 \times B S \times \sin (B S)$, and stochastic component is represented with the known random error $(\epsilon)$ that is normally distributed with the mean equal to zero and standard deviation equal to $2 \mathrm{~cm}(\mathcal{N}(0,2))$. This random error can be termed irreducible error (James et al., 2017), since it is inherent to the true DGP. As will be demonstrated shortly, models that perform better than this irreducible error are said to overfit. In other words, models are jumping to the noise.

The objective of causal inference or explanatory modeling is to estimate the $f(X)$ (estimate is indicated with the hat symbol: $\hat{f}(x))$ or to understand the underlying DGP. With the predictive analysis, the goal is to find the best estimate of $Y$ or $\hat{y}$. The underlying DGP is treated as a black box.

To demonstrate a concept of overfitting, we are going to generate two samples ( $\mathrm{N}=35$ observations) from the DGP with BS ranging from 0.8 to 2.5. These samples are training and testing sample (Figure 12). Training sample is used to train the prediction model, while testing sample will be used as a holdout sample for evaluating model performance on the unseen data.

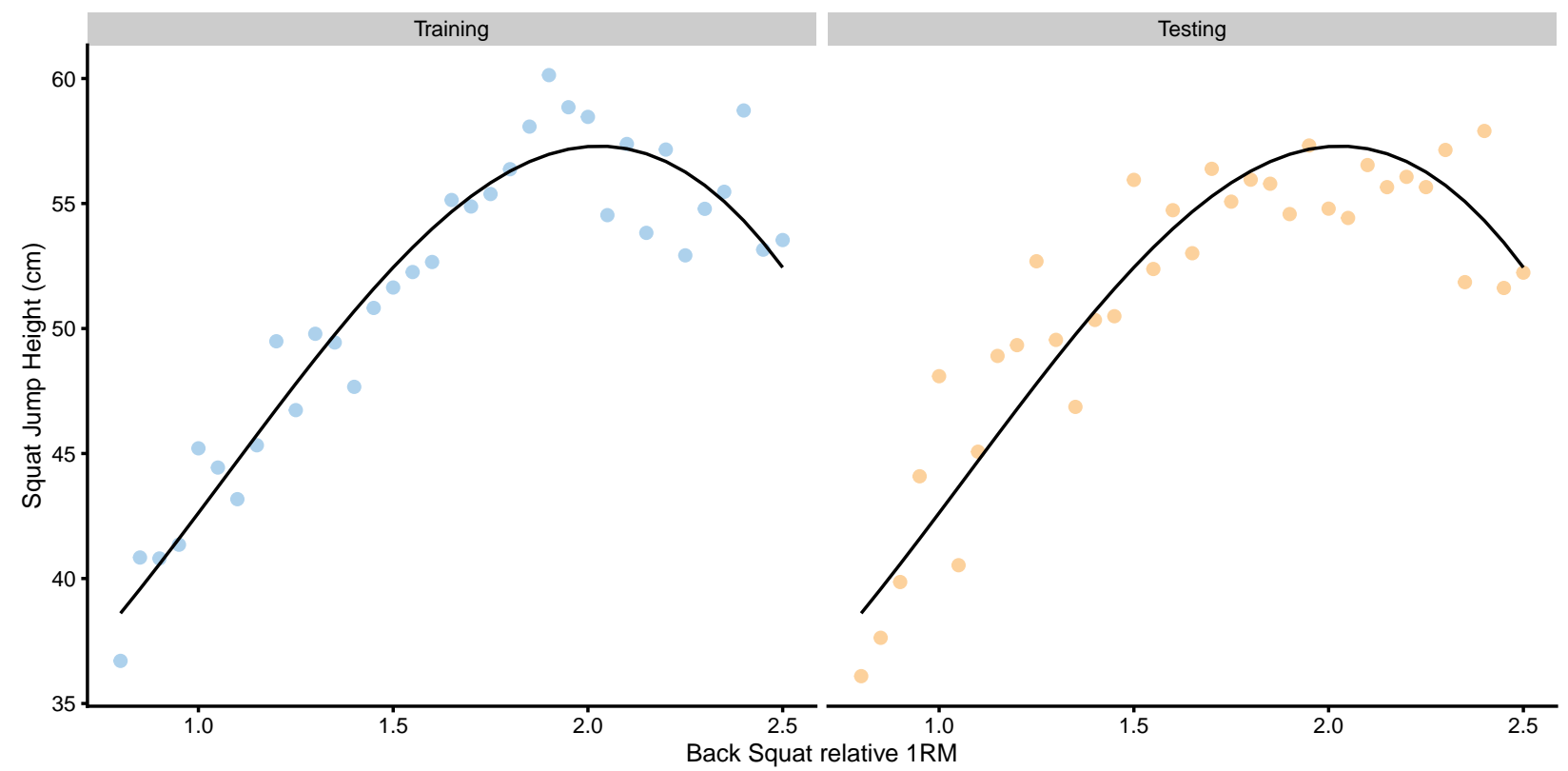

\section{Figure 12. Black line represent systematic component of the DGP and it is equal for both training and testing samples}

Model used to predict SJ from BS will be polynomial linear regression. Equation 25 explains first, second, and third degree polynomial linear regression function and provides a form for n-degree polynomials. Please, note that first degree polynomial function represents simple linear regression. 


$$
\begin{aligned}
& \hat{y_{i}}=\hat{\beta}_{0}+\hat{\beta}_{1} x_{i}^{1} \\
& \hat{y_{i}}=\hat{\beta}_{0}+\hat{\beta}_{1} x_{i}^{1}+\hat{\beta}_{2} x_{i}^{2} \\
& \hat{y}_{i}=\hat{\beta}_{0}+\hat{\beta}_{1} x_{i}^{1}+\hat{\beta}_{2} x_{i}^{2}+\hat{\beta}_{3} x_{i}^{3} \\
& \hat{y}_{i}=\hat{\beta}_{0}+\hat{\beta}_{1} x_{i}^{1}+\cdots+\hat{\beta}_{n} x_{i}^{n}
\end{aligned}
$$

Increasing polynomial degrees increases the flexibility of the polynomial regression model, and thus can represent tuning parameter that we can select based on the model performance. In other words, we might be interested in finding polynomial degree that minimized model error (or maximize model fit). Figure 13 contains model performance on the training data for polynomial degrees ranging from 1 to 20.
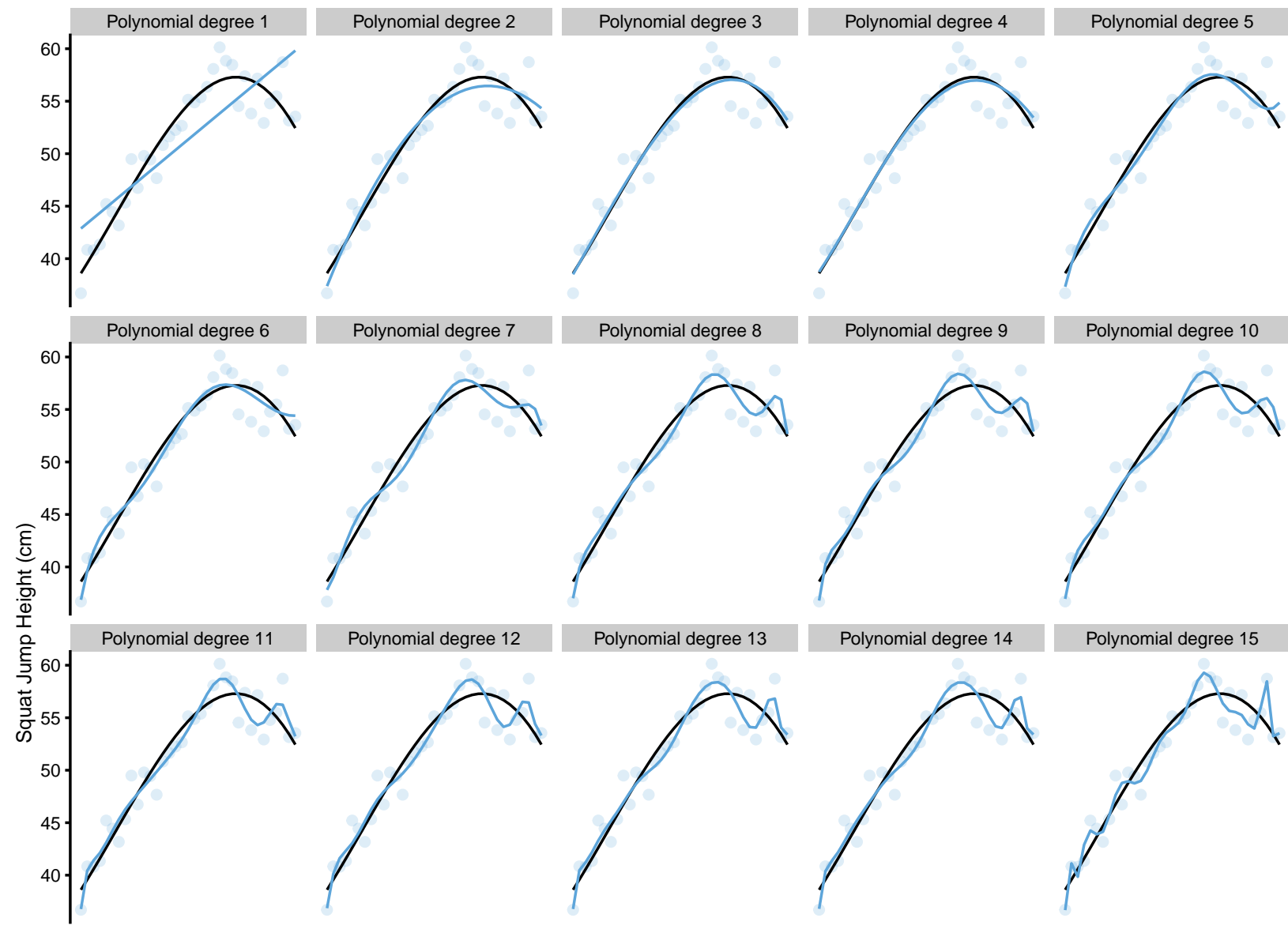

Polynomial degree 8

Polynomial degree 9
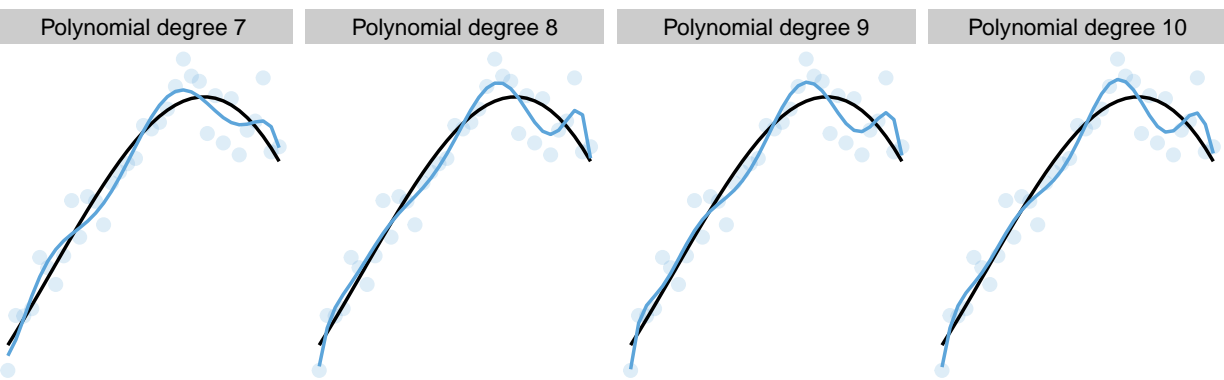

Polynomial degree 12
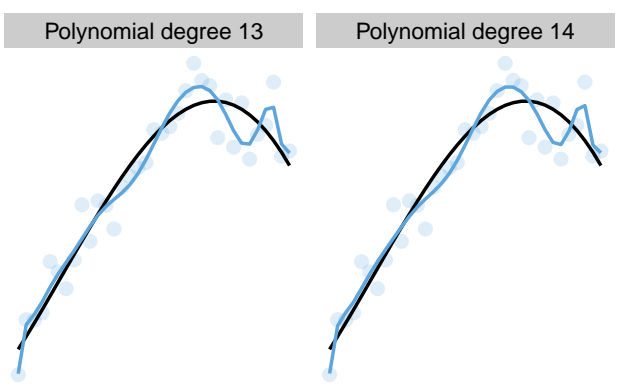

Polynomial degree 15
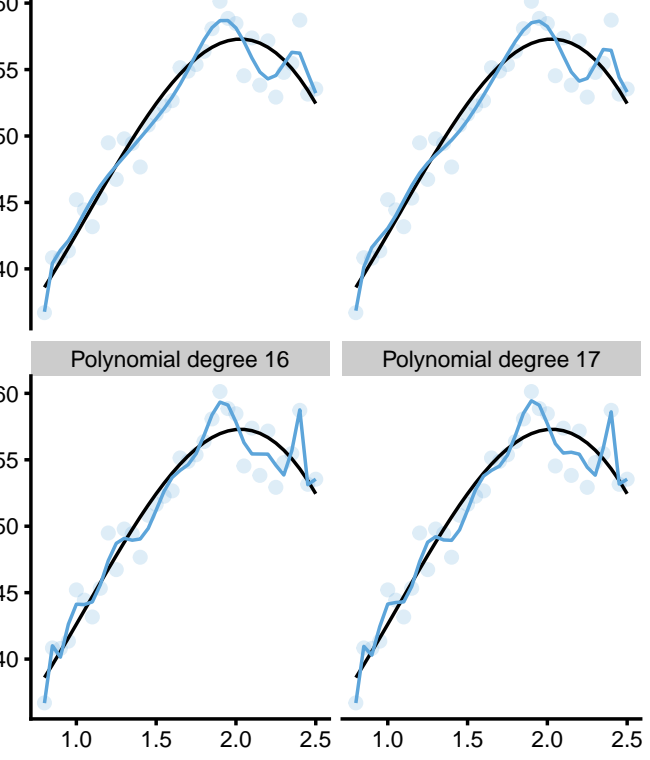

Polynomial degree 17
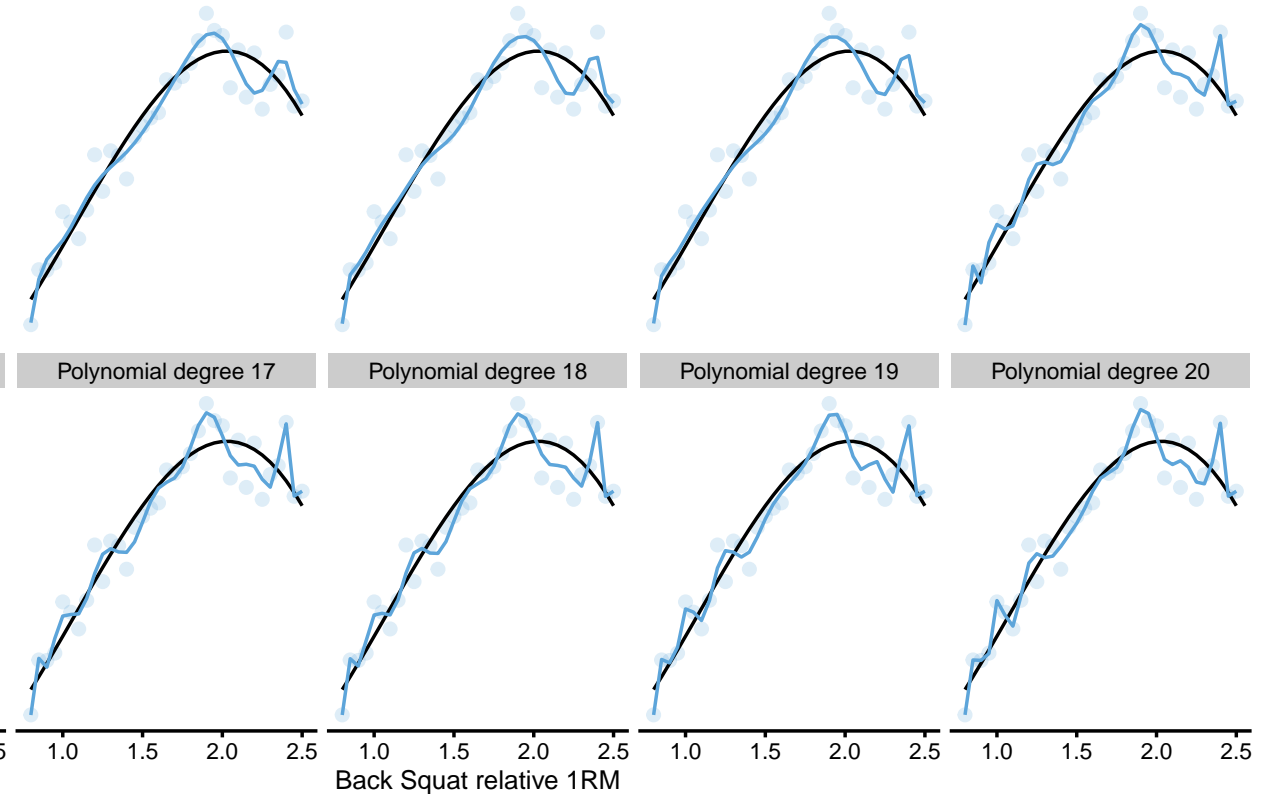

Figure 13. Model fit with varying polynomial degrees. More degrees equals better model fit 
As can be seen from the Figure 13, the more flexible the model (or the higher the polynomial degree) the better it fits the data. But how do these models perform on the unseen, testing data sample? In order to quantify model performance, RMSE metric is used. Figure 14 demonstrates performance of the polynomial regression model on the training and testing data sample across different polynomial degrees.

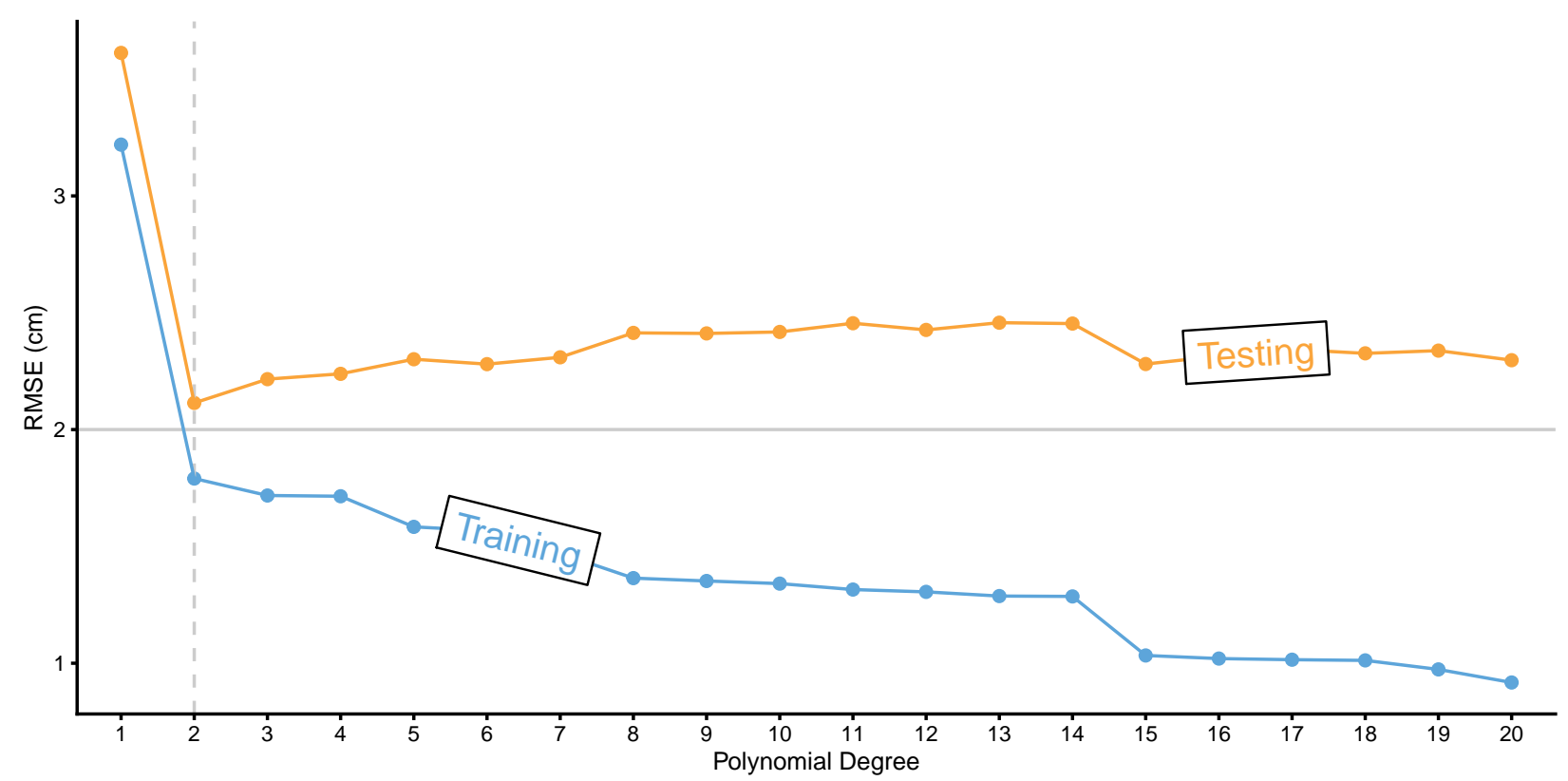

Figure 14. Testing and training errors across varying polynomial degrees. Model error is estimated with the RMSE metric, while polynomial degree represents tuning or flexibility parameter of the model. As can be noted from the image, better training performance doesn't imply better testing performance. Vertical dashed line represents the polynomial degree at which testing error is lowest.

Polynomial degrees on the right of the vertical dashed line are said to overfit the data, while polynomial degree on the left are said to underfit the data

As can be seen from the Figure 14, models with higher polynomial degrees tend to overfit (indicated by performance better than the known irreducible error $\epsilon$ visualized with the horizontal line at $2 \mathrm{~cm}$ ). Performance on the training data sample improves as the polynomial degrees increase, which is not the case with the performance on the testing data sample. There is clearly the best polynomial degree that has the best predictive performance on the unseen data. Polynomial degrees on the left of the vertical dashed line are said to underfit, while polynomial degrees on the right are said to overfit.

The take home message is that predictive performance on the training data can be too optimistic, and for evaluating predictive performance of the model, unseen data must be used, otherwise the model might overfit.

\subsubsection{Cross-Validation}

In order to evaluate predictive performance of the model, researchers usually remove some percent of data to be used as a testing or holdout sample. Unfortunately, this is not always possible (although it is recommended, particularly to evaluate final model performance, especially when there are multiple models and model tuning). One solution to this problems is cross-validation technique (James et al., 2017; Yarkoni \& Westfall, 2017; Kuhn \& Johnson, 2018). There are numerous variations of the cross-validation, but the simplest one is $n$-fold cross validation (Figure 15). N-fold cross validation involve splitting the data into 5 to 10 equal folds and using one fold as a testing or hold-out sample while performing model training on the other folds. This is 
repeated over $\mathrm{N}$-iteration (in this case 5 to 10) and the model performance is averaged to get cross-validated model performance.

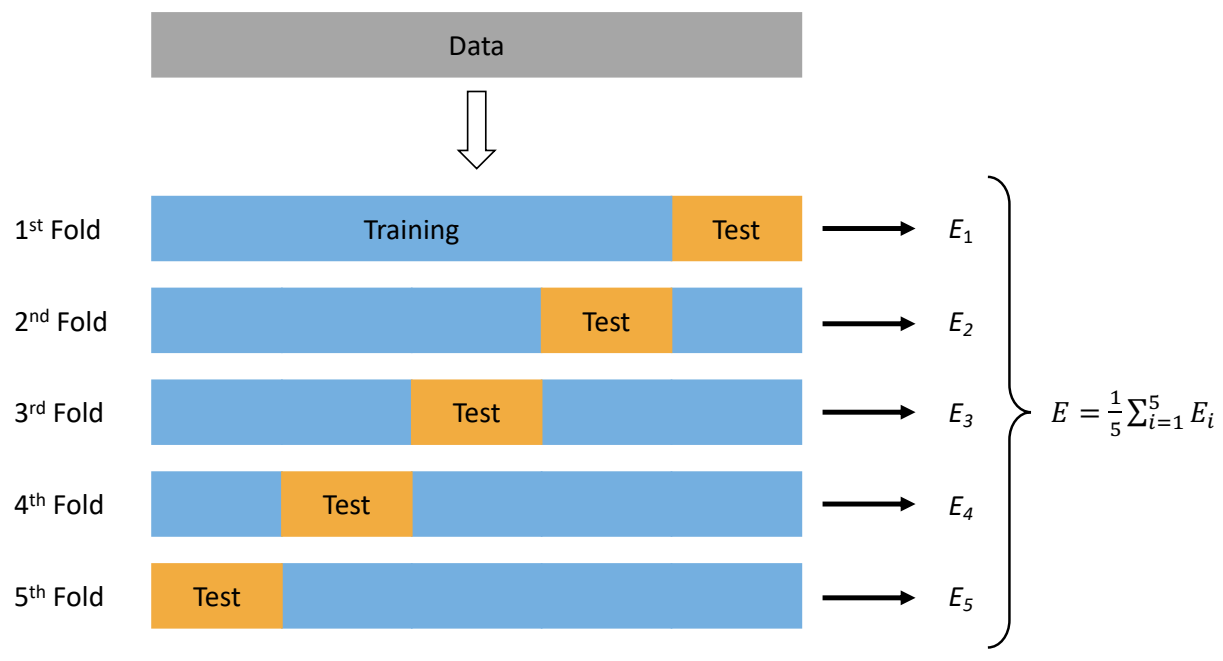

Figure 15. Cross-Validation.

With predictive analysis and machine learning, different model's tuning parameters are evaluated (as well as multiple different models) to estimate the one that gives the best predictive performance. It is thus important to utilize techniques such as cross-validation to avoid overfitting and too optimistic model selection.

Certain models, such as lasso, ridge regression, and elastic-net implement regularization parameters that penalizes the model complexity and are used as a tuning variable (James et al., 2017; Yarkoni \& Westfall, 2017; Kuhn \& Johnson, 2018). This is useful in situations when there are a lot of predictors, and it is easy to overfit the model. Selecting the best regularization parameter that has the best cross-validated performance helps in simplifying the model and avoiding the overfit. These topics are beyond the scope of the current paper, and interested readers are directed to references provided at the end of the paper.

\subsubsection{Bias-Variance decomposition and trade-off}

Prediction error can be decomposed into two components, reducible and irreducible error ${ }^{15}$. Reducible error is the model that can be reduced with a better model, while irreducible error is the unknown error inherent to the DGP itself (James et al., 2017). Reducible error can be further divided into bias and variance (Figure 16 and equation 26).

$$
\begin{aligned}
& \text { Prediction error }=\text { Reducible error }+ \text { Irreducible error } \\
& \text { Prediction error }=\left(\text { Bias }^{2}+\text { Variance }\right)+\text { Irreducible error }
\end{aligned}
$$

Bias represents constant or systematic error, which is introduced by approximating a real-life problem, which may be extremely complicated, by a much simpler model (James et al., 2017). Variance represents variable or random error, and refers to the amount by which model parameters would change if we estimated it by using a different training data set (James et al., 2017).

\footnotetext{
${ }^{15}$ As it will be explained in "Statistical inference" section, there are two kinds of uncertainty: aleatory and epistemic. Aleatory uncertainty is inherent randomness and it is usually represented as irreducible error. Epistemic uncertainty is due to the lack of knowledge or information, which can be considered reducible error. In other words, better models or models with more information will be able to reduce prediction error by reducing the reducible or epistemic uncertainty. The upper ceiling of the predictive performance is limited by irreducible error (which is unknown). Please refer to the "Statistical inference" section for more information.
} 
A

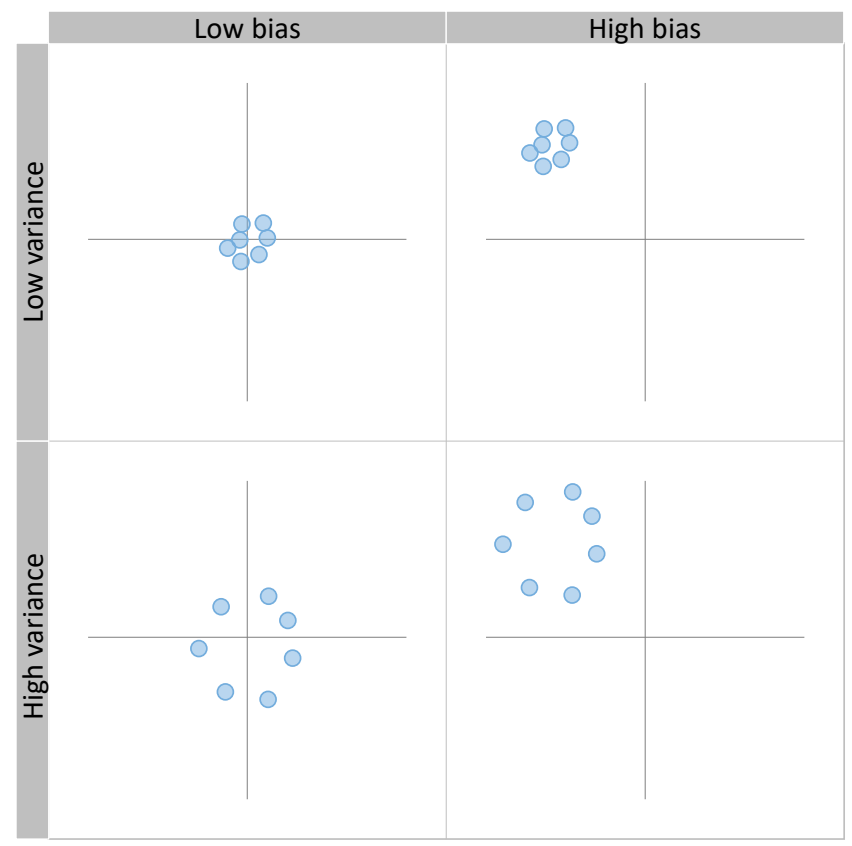

B

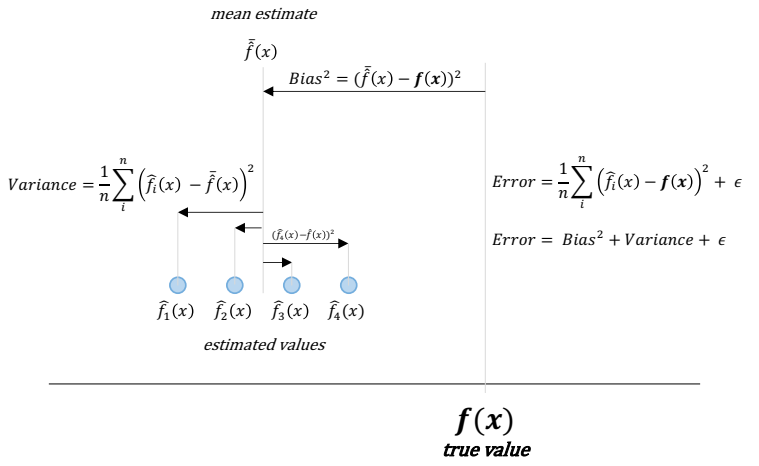

Figure 16. Bias and variance decomposition of the error. A. Visual representation of bias and variance using the shooting target. B. Error can be decomposed to bias, variance, and irreducible error, where bias is constant or systematic error and variance is variable or random error.

Since we know the true relationship between BS and SJ, we can generate the data from DGP multiple times (i.e. 100 times) and estimate model's bias and variance. This is done for one $x$ value - in this case for $B S=1.75$, where true $Y$ or $S J=55.83 \mathrm{~cm}$. Total error is estimated by using mean-square-error (or MSE) which quantifies variance (equation 27 and panel B on Figure 16).

$$
M S E=\frac{1}{n} \sum_{i=1}^{n}\left(y_{i}-\hat{y}_{i}\right)^{2}
$$

The results of this simulation and decomposition of the prediction error to bias and variance is depicted on the Figure 17. 


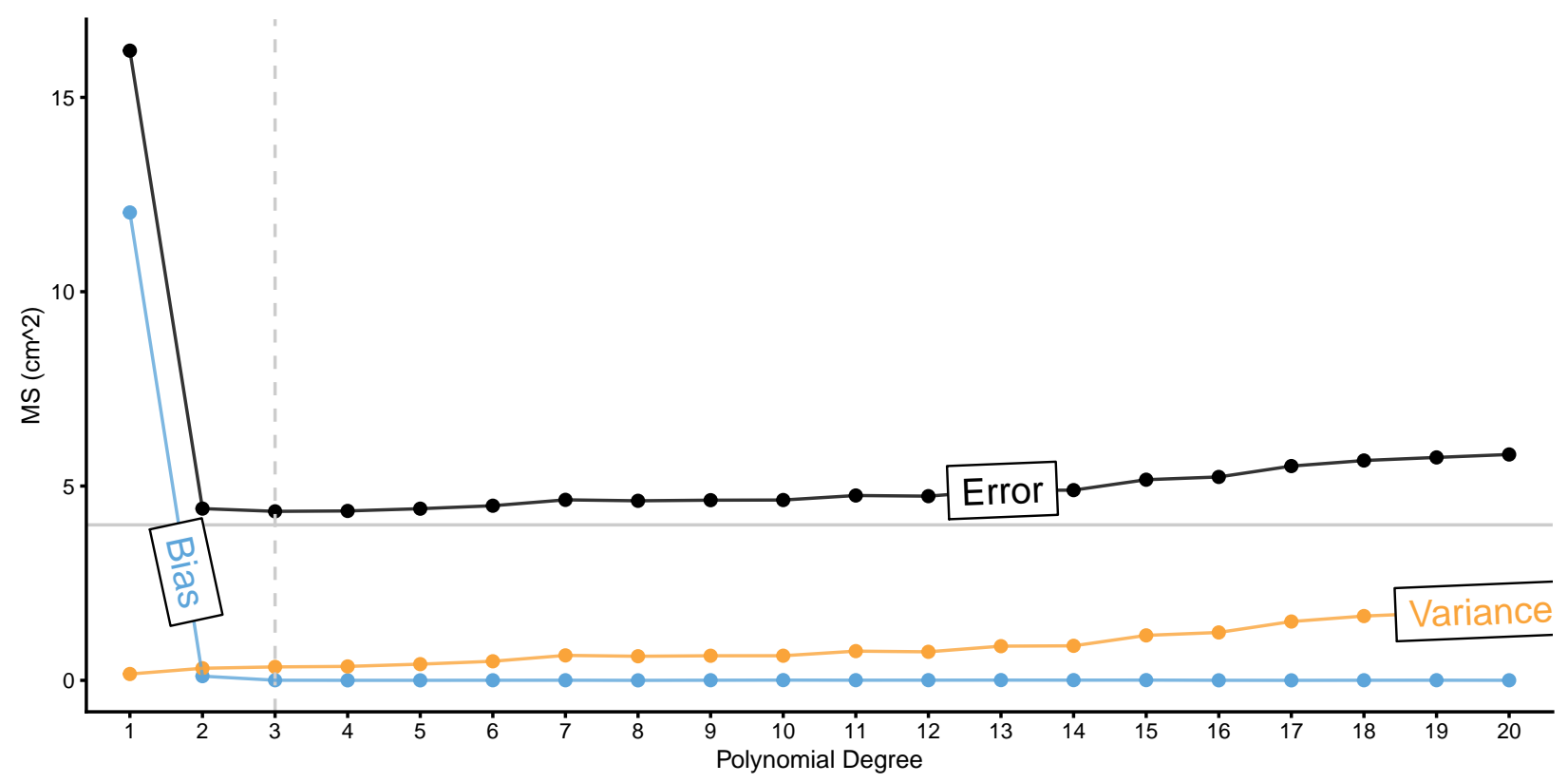

Figure 17.Bias and Variance error decomposition. Prediction error is indicated with the black line, which is decomposed to bias (blue line) and variance (orange line). Horizontal grey line represents irreducible error which is known since we have generated the data. Vertical dashed line represents polynomial degree

with the lower prediction error

The conclusion is that there is a bias-variance trade-off, as well as the optimal balance between the two that maximizes model performance. Some models, such as linear regression as inherently more biased (i.e. linear regression models try to fit linear line to the data) and some provide much more variance. Unfortunately, there is no such thing as free lunch (Yarkoni \& Westfall, 2017; Kuhn \& Johnson, 2018), which implies that there is no single model that is the best across all different sets of problems. One needs to evaluate multiple models ${ }^{16}$ to estimate which one is the best for a particular problem at hand.

\subsubsection{Interpretability}

As explained, predictive models put predictive performance over explanation of the underlying DGP mechanism (which is treated as a black box). However, sometimes we might be interested in which predictor is the most important, how do predictions change when particular predictor changes, or why ${ }^{17}$ model made a particular prediction for a case of interest (Ribeiro, Singh \& Guestrin, 2016; Molnar, 2018; Kuhn \& Johnson, 2018). Model interpretability can be defined as "the degree to which a human can understand the cause of a decision" (Miller, 2017; Molnar, 2018). Some models are more inherently interpretable (e.g. linear regression) and some are indeed very complex and hard to interpret (e.g. random forest or neural networks). For this reason, there are model-agnostic techniques that can help increase model interpretability.

\footnotetext{
${ }^{16}$ Or to utilize subject matter knowledge needed to select the model. More about this topic can be found in the "Subject matter knowledge" section of this paper

${ }^{17}$ With the recent laws such as European's DGPR, predictive models needs to be able to explain or provide explanation why particular decision or prediction has been made. Christoph Molnar (Molnar, 2018) explains the need for model interpretability with one interesting example: "By default, machine learning models pick up biases from the training data. This can turn your machine learning models into racists that discriminate against protected groups. Interpretability is a useful debugging tool for detecting bias in machine learning models. It might happen that the machine learning model's, you have trained for, automatic approval or rejection of credit applications discriminates against a minority. Your main goal is to grant loans only to people who will eventually repay them. The incompleteness of the problem formulation in this case lies in the fact that you not only want to minimize loan defaults, but are also obliged not to discriminate on the basis of certain demographics. This is an additional constraint that is part of your problem formulation (granting loans in a low-risk and compliant way) that is not covered by the loss function the machine learning model was optimized for." (p.11)
} 
Excellent book and R package by Christoph Molnar (Molnar, Bischl \& Casalicchio, 2018; Molnar, 2018) demonstrates a few model-agnostic interpretable techniques. One such technique is understating which predictor is the most important. This is done by perturbing one predictor and estimating the change in model performance. The predictor whose perturbing causes the biggest change in model performance can be considered the most important (Molnar, 2018; Kuhn \& Johnson, 2018).

One might be interested in how the predicted outcome changes when particular predictor changes. Techniques such as partial dependence plot (PDP), individual conditional expectation (ICE) and accumulated local effects (ALE) can be helpful in interpreting the effect of particular predictor on predicted outcome (Goldstein et al., 2013; Molnar, Bischl \& Casalicchio, 2018; Molnar, 2018; Zhao \& Hastie, 2019). These techniques are utilized later in this paper.

It is important to keep in mind that these model-agnostic explanations should not be automatically treated as causal explanations (Pearl \& Mackenzie, 2018; Pearl, 2019), but as mere association and descriptive analysis that still can be useful in understanding and interpreting the underlying predictive black-box. According to Judea Pearl (Pearl \& Mackenzie, 2018; Pearl, 2019), prediction models should belong to the first level of ladder of causation, which represents simple "curve fitting". Although in under special conditions these techniques can have causal interpretation (Zhao \& Hastie, 2019).

The distinctions, similarities and issues between predictive modelling, machine learning and causal inference is currently hot topic in debates between machine learning specialists, statisticians and philosophers of science and it is beyond the scope of this paper to delve into the debate. Interested readers are directed towards work by Miguel Hernan (Hernán, 2016, 2017, 2018; Hernán, Hsu \& Healy, 2019; Hernán \& Robins), Judea Pearl (Pearl, 2009, 2019; Pearl, Glymour \& Jewell, 2016; Pearl \& Mackenzie, 2018), Samantha Kleinberg (Kleinberg, 2015, 2018) and others (Breiman, 2001; Shmueli, 2010; Kleinberg, Liang \& Mullainathan, 2017; Yarkoni \& Westfall, 2017; Saddiki \& Balzer, 2018; Watts et al., 2018). The next "Causal Inference" section introduces the causal inference as a specific task of statistical modelling.

\subsubsection{Magnitude-based prediction estimators}

Similar to the magnitude-based estimators from "Describing relationship between two variables" section, one can utilize target variable SESOI to get magnitude-based estimates of predictive performance of the model. Rather than utilizing RSE as an estimate of the model fit in the training data, one can utilize cross-validated RMSE (cvRMSE), SESOI to cvRMSE, as well as cross-validated equivalence (cvEquivalence).

Continuing with the squat jump and relative squat 1RM, one can assume that the SESOI in the squat jump is $\pm 1 \mathrm{~cm}$. For the sake of example, we can feature engineer (Kuhn \& Johnson, 2018, 2019) relative squat $1 \mathrm{RM}$ variable to include all 20 degree polynomials. This way, we have created 20 predictor variables. To avoid overfitting, elastic-net model (Friedman, Hastie \& Tibshirani, 2010) implemented in the caret package (Kuhn et al., 2018; Kuhn \& Johnson, 2018) is utilized, as well as repeated cross-validation involving 3 splits repeated 10 times. Predictive model performance is evaluated by using cross-validated RMSE, together with magnitude-based performance estimators.

Elastic-net model represents regression method that linearly combines the $L 1$ and $L 2$ penalties of the lasso and ridge methods, or alpha and lambda tuning parameters (Friedman, Hastie \& Tibshirani, 2010; James et al., 2017; Kuhn \& Johnson, 2018). Total of nine combinations of tuning parameters is evaluated using aforementioned repeated cross-validation, and the model with minimal cvRMSE is selected as the best one. Performance metrics of the best model are further reported. Table 16 contains cross-validated best model performance metrics together with model performance on the training data set.

Table 16. Common predictive metrics and magnitude-based predictive metrics. Metrics starting with 'cv' indicate cross-validated performance metrics. Metrics without 'cv' indicate performance metrics on the training data set, which is often more optimistic 


\begin{tabular}{lrrrrrr}
\hline SESOI $(\mathrm{cm})$ & cvRMSE $(\mathrm{cm})$ & SESOI to cvRMSE & cvEquivalence & RMSE $(\mathrm{cm})$ & SESOI to RMSE & Equivalence \\
\hline \pm 1 & 2.16 & 0.93 & 0.36 & 1.85 & 1.08 & 0.41 \\
\hline
\end{tabular}

Utilizing apriori known SESOI gives us practical anchor to evaluate predictive model performance. Reported SESOI to cvRMSE (0.93) as well as cvEQuivalence (0.36) indicate very poor predictive performance of the model. In practical terms, utilizing relative squat 1RM doesn't produce practically meaningful predictions given SESOI of $\pm 1 \mathrm{~cm}$ and the model as well as the data sample utilized.

Model performance can be visualized using the training data set (Figure 18). Equivalence estimator, for both cross-validate estimate and training data performance estimate, utilized SD of the residuals and provided SESOI. Grey band on panels A and B on Figure 18 represents SESOI, and as can be visually inspected, model residuals are much wider than the SESOI, indicating poor practically significant performance.
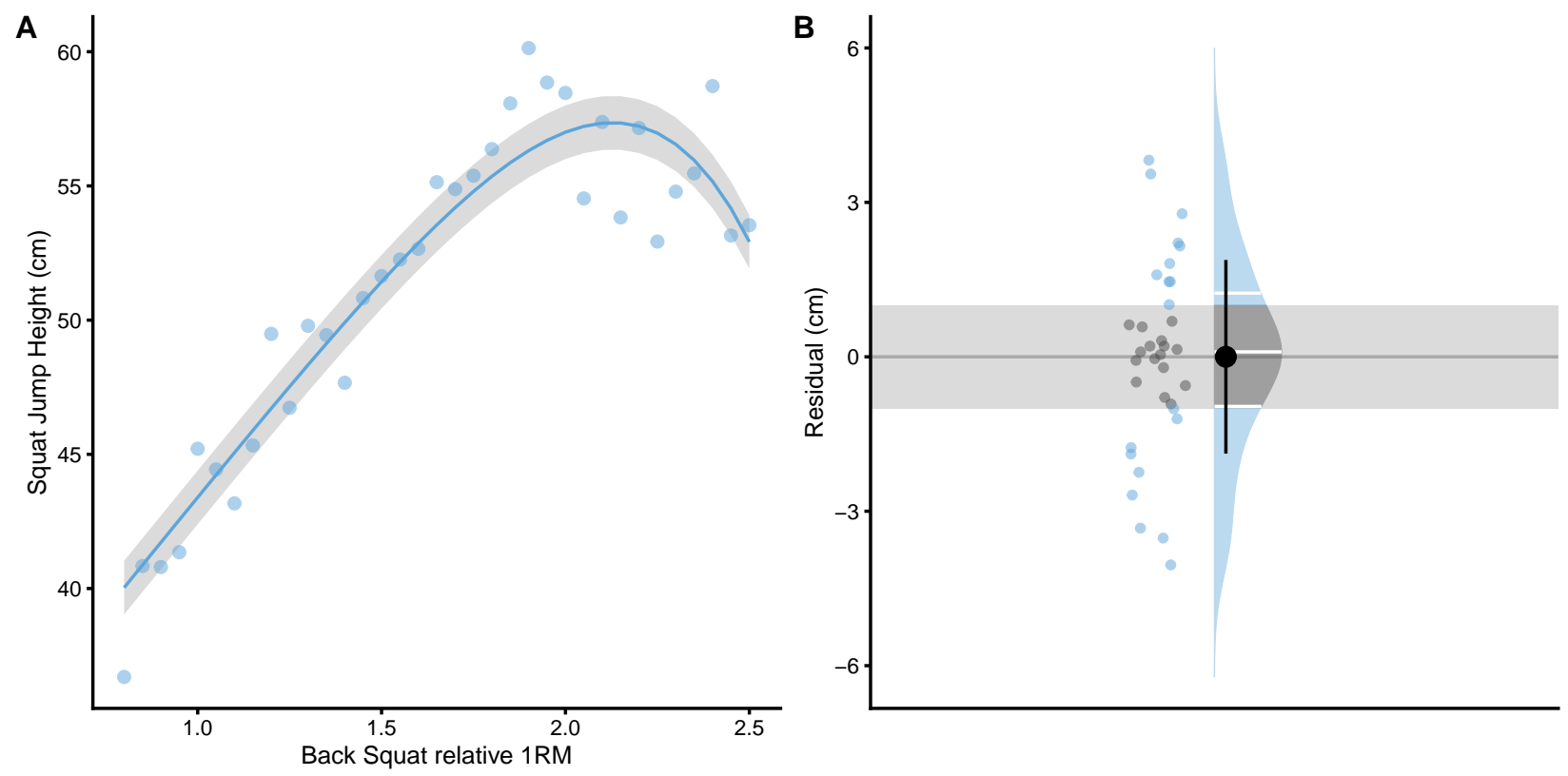

Figure 18. Model performance on the training data set. A. Model with the lowest cvRMSE is selected. SESOI is depicted as grey band around the model prediction (blue line). B. Residuals scatter plot. Residuals outside of the SESOI band (grey band) indicate prediction which error is practically significant.

Equivalence represents proportion of residuals inside the SESOI band

Predictive tasks are focusing on providing the best predictions on the novel or unseen data without much concern about the underlying DGP. Predictive model performance can be evaluated by using magnitude-based approach to give insights into practical significance of the predictions. These magnitude-based prediction estimators, can be used to complement explanatory or causal inference tasks, rather than relying solely on the group-based and average-based estimators. This topic is further discussed in the "Prediction as a complement to causal inference" section of this paper.

\subsection{Causal Inference}

Does playing basketball makes one taller? This is a an example of a causal question. Wrestling with the concept of causality, as a philosophical construct is outside the scope of this paper (and the author 
too), but I will define it using the counterfactual theory or potential outcomes perspective (Gelman, 2011; Angrist \& Pischke, 2015; Kleinberg, 2015; Pearl \& Mackenzie, 2018; Hernán, Hsu \& Healy, 2019) that define causes in terms of how things would have been different had the cause not occurred, as well as from causality-as-intervention perspective (Gelman, 2011), which necessitates clearly defined interventions (Hernán \& Taubman, 2008; Hernán, 2016, 2018). In other words, would someone be shorter if basketball was never trained?

There are two broad classes of inferential question that focus on what if and why: forward causal inference ("What might happen if we do X?") and reverse causal inference ("What causes $Y$ ? Why?") (Gelman, 2011). Forward causation is more clearly defined problem, where the goal is to quantify the causal effect of treatment. Questions of forward causation are most directly studied using randomization (Gelman, 2011) and are answered from the above mentioned causality-as-intervention and counterfactual perspectives. Reverse causation is more complex and it is more related to explaining the causal chains using the system-variable approach. Article by Andrew Gelman (Gelman, 2011) provides great overview of the most common causal perspectives, out of which I will mostly focus on forward causation.

\subsubsection{Necessary versus sufficient causality}

Furthermore, we also need to distinguish between four kinds of causation (Kleinberg, 2015; Pearl \& Mackenzie, 2018): necessary causation, sufficient causation and neither or both. For example, if someone says that A causes B, then:

- If A is necessary for B, it means that if A never happened (counterfactual reasoning), then B will never happen. Or, in other words, B can never happen without A. But sufficient causality also means that A can happen without B happening.

- If A is sufficient for B, it means that if you have A, you will always have B. In other words, B always follows A. However, sometimes B can happen without A

- If A is neither sufficient nor necessary for B, then sometimes when A happens B will happen. B can also happen without A.

- If $\mathrm{A}$ is both necessary and sufficient for B, then B will always happen after A, and B will never happen without A.

Table 17 contains summary of the above necessary and sufficient causality. In all four types of causation, the concept of counterfactual reasoning is invoked.

Table 17. Four kinds of causation

\begin{tabular}{lllll}
\hline Cause & Necessary & Sufficient & Neither & Both \\
\hline A happens & B might happen & B always happen & B might happen & B always happen \\
A doesn't happen & B never happens & B might happen & B might happen & B never happens \\
\hline
\end{tabular}

Although the causal inference is a broad area of research, philosophical discussion and conflicts, there are a few key concepts that need to be introduced to get the big picture and understand the basics behind the aims of causal inference. Let's start with an example involving the aforementioned question whether playing basketball makes one taller.

\subsubsection{Observational data}

In order to answer this question, we have collected height data (expressed in $\mathrm{cm}$ ) for the total of $\mathrm{N}=30$ athletes, of which $\mathrm{N}=15$ play basketball, and $\mathrm{N}=15$ don't play basketball (Table 18). Playing basketball can be considered intervention or treatment, in which causal effect we are interested in. Basketball players are considered intervention or treatment group and those without the treatment are considered comparison or control group 
Table 18. Height in the treatment and control groups

\begin{tabular}{llr}
\hline Athlete & Treatment & Height $(\mathrm{cm})$ \\
\hline Athlete 11 & Basketball & 213 \\
Athlete 01 & Basketball & 210 \\
Athlete 13 & Basketball & 210 \\
Athlete 19 & Basketball & 209 \\
Athlete 27 & Basketball & 208 \\
Athlete 09 & Basketball & 207 \\
Athlete 05 & Basketball & 206 \\
Athlete 15 & Basketball & 205 \\
Athlete 25 & Basketball & 200 \\
Athlete 17 & Basketball & 198 \\
Athlete 07 & Basketball & 192 \\
Athlete 29 & Basketball & 191 \\
Athlete 21 & Basketball & 187 \\
Athlete 03 & Basketball & 187 \\
Athlete 23 & Basketball & 184 \\
Athlete 20 & Control & 204 \\
Athlete 26 & Control & 196 \\
Athlete 12 & Control & 195 \\
Athlete 24 & Control & 186 \\
Athlete 18 & Control & 185 \\
Athlete 14 & Control & 184 \\
Athlete 10 & Control & 184 \\
Athlete 08 & Control & 182 \\
Athlete 30 & Control & 181 \\
Athlete 06 & Control & 177 \\
Athlete 04 & Control & 177 \\
Athlete 16 & Control & 174 \\
Athlete 22 & Control & 170 \\
Athlete 02 & Control & 168 \\
Athlete 28 & Control & 160 \\
\hline & &
\end{tabular}

Using descriptive estimators introduced in the "Description" section, one can quickly calculate the group means and SDs as well as their difference (Table 19). But does mean difference between basketball and control represent average causal effect? No, unfortunately not!

Table 19. Descriptive analysis of the groups

\begin{tabular}{lrr}
\hline & Mean $(\mathrm{cm})$ & SD $(\mathrm{cm})$ \\
\hline Basketball & 200.48 & 9.91 \\
Control & 181.61 & 11.47 \\
Difference & 18.87 & 15.16 \\
\hline
\end{tabular}

\subsubsection{Potential outcomes or counterfactuals}

To explain why this is the case, we need to imagine alternate counterfactual reality. What is needed are two potential outcomes: Height ${ }_{0}$, which represents height of the person if one doesn't train basketball, and 
Height $_{1}$ which represents height of the person if basketball is being played (Table 20). As can be guessed, the Basketball group has known Height ${ }_{1}$, but unknown Height $t_{0}$ and vice versa for the Control group.

Table 20. Counterfactuals of potential outcomes that are unknown

\begin{tabular}{|c|c|c|c|c|c|}
\hline Athlete & Treatment & Height_0 $(\mathrm{cm})$ & Height_1 $(\mathrm{cm})$ & Height $(\mathrm{cm})$ & Causal Effect $(\mathrm{cm})$ \\
\hline Athlete 11 & Basketball & ??? & 213 & 213 & ??? \\
\hline Athlete 01 & Basketball & $? ? ?$ & 210 & 210 & ??? \\
\hline Athlete 13 & Basketball & $? ? ?$ & 210 & 210 & ??? \\
\hline Athlete 19 & Basketball & $? ? ?$ & 209 & 209 & $? ? ?$ \\
\hline Athlete 27 & Basketball & $? ? ?$ & 208 & 208 & $? ? ?$ \\
\hline Athlete 09 & Basketball & $? ? ?$ & 207 & 207 & $? ? ?$ \\
\hline Athlete 05 & Basketball & $? ? ?$ & 206 & 206 & $? ? ?$ \\
\hline Athlete 15 & Basketball & $? ? ?$ & 205 & 205 & $? ? ?$ \\
\hline Athlete 25 & Basketball & $? ? ?$ & 200 & 200 & $? ? ?$ \\
\hline Athlete 17 & Basketball & $? ? ?$ & 198 & 198 & $? ? ?$ \\
\hline Athlete 07 & Basketball & $? ? ?$ & 192 & 192 & $? ? ?$ \\
\hline Athlete 29 & Basketball & $? ? ?$ & 191 & 191 & $? ? ?$ \\
\hline Athlete 21 & Basketball & $? ? ?$ & 187 & 187 & $? ? ?$ \\
\hline Athlete 03 & Basketball & $? ? ?$ & 187 & 187 & $? ? ?$ \\
\hline Athlete 23 & Basketball & $? ? ?$ & 184 & 184 & $? ? ?$ \\
\hline Athlete 20 & Control & 204 & $? ? ?$ & 204 & $? ? ?$ \\
\hline Athlete 26 & Control & 196 & $? ? ?$ & 196 & $? ? ?$ \\
\hline Athlete 12 & Control & 195 & $? ? ?$ & 195 & $? ? ?$ \\
\hline Athlete 24 & Control & 186 & $? ? ?$ & 186 & $? ? ?$ \\
\hline Athlete 18 & Control & 185 & $? ? ?$ & 185 & $? ? ?$ \\
\hline Athlete 14 & Control & 184 & ??? & 184 & $? ? ?$ \\
\hline Athlete 10 & Control & 184 & ??? & 184 & ??? \\
\hline Athlete 08 & Control & 182 & ??? & 182 & $? ? ?$ \\
\hline Athlete 30 & Control & 181 & ??? & 181 & $? ? ?$ \\
\hline Athlete 06 & Control & 177 & ??? & 177 & ??? \\
\hline Athlete 04 & Control & 177 & ??? & 177 & $? ? ?$ \\
\hline Athlete 16 & Control & 174 & ??? & 174 & ??? \\
\hline Athlete 22 & Control & 170 & ??? & 170 & ??? \\
\hline Athlete 02 & Control & 168 & ??? & 168 & $? ? ?$ \\
\hline Athlete 28 & Control & 160 & ??? & 160 & $? ? ?$ \\
\hline
\end{tabular}

Unfortunately, these potential outcomes are unknown, and thus individual causal effects. We just do not know what might have happened to individual outcomes in counterfactual world (i.e. alternate reality). A good control group serves as a proxy to reveal what might have happened on average to the treated group in the counterfactual world where they are not treated. Since the basketball data is simulated, the exact DGP is known (the true systematic or fixed causal effect of playing basketball on height is $0 \mathrm{~cm}$, with random effect normally distributed with SD equal to $1 \mathrm{~cm}$ ), which again demonstrates the use of simulations as a great learning tool, in this case understanding the underlying causal mechanisms (Table 21). Individual causal effect in this case is the difference between two potential outcomes: Height $t_{1}$ and Height . $_{\text {. }}$

Table 21. Simulated causal effects and known counterfactuals 


\begin{tabular}{|c|c|c|c|c|c|}
\hline Athlete & Treatment & Height_0 $(\mathrm{cm})$ & Height_1 $(\mathrm{cm})$ & Height $(\mathrm{cm})$ & Causal Effect $(\mathrm{cm})$ \\
\hline Athlete 11 & Basketball & 215 & 213 & 213 & -1.26 \\
\hline Athlete 01 & Basketball & 209 & 210 & 210 & 1.29 \\
\hline Athlete 13 & Basketball & 210 & 210 & 210 & -0.03 \\
\hline Athlete 19 & Basketball & 207 & 209 & 209 & 1.55 \\
\hline Athlete 27 & Basketball & 206 & 208 & 208 & 2.19 \\
\hline Athlete 09 & Basketball & 207 & 207 & 207 & 0.82 \\
\hline Athlete 05 & Basketball & 207 & 206 & 206 & -1.38 \\
\hline Athlete 15 & Basketball & 204 & 205 & 205 & 1.09 \\
\hline Athlete 25 & Basketball & 197 & 200 & 200 & 2.20 \\
\hline Athlete 17 & Basketball & 199 & 198 & 198 & -1.34 \\
\hline Athlete 07 & Basketball & 191 & 192 & 192 & 0.32 \\
\hline Athlete 29 & Basketball & 193 & 191 & 191 & -1.32 \\
\hline Athlete 21 & Basketball & 188 & 187 & 187 & -1.35 \\
\hline Athlete 03 & Basketball & 187 & 187 & 187 & -0.53 \\
\hline Athlete 23 & Basketball & 184 & 184 & 184 & -0.27 \\
\hline Athlete 20 & Control & 204 & 203 & 204 & -0.94 \\
\hline Athlete 26 & Control & 196 & 197 & 196 & 0.91 \\
\hline Athlete 12 & Control & 195 & 196 & 195 & 0.91 \\
\hline Athlete 24 & Control & 186 & 186 & 186 & -0.02 \\
\hline Athlete 18 & Control & 185 & 184 & 185 & -0.44 \\
\hline Athlete 14 & Control & 184 & 184 & 184 & -0.73 \\
\hline Athlete 10 & Control & 184 & 183 & 184 & -0.99 \\
\hline Athlete 08 & Control & 182 & 183 & 182 & 0.80 \\
\hline Athlete 30 & Control & 181 & 182 & 181 & 0.05 \\
\hline Athlete 06 & Control & 177 & 177 & 177 & 0.19 \\
\hline Athlete 04 & Control & 177 & 179 & 177 & 2.10 \\
\hline Athlete 16 & Control & 174 & 175 & 174 & 1.49 \\
\hline Athlete 22 & Control & 170 & 170 & 170 & 0.38 \\
\hline Athlete 02 & Control & 168 & 169 & 168 & 1.28 \\
\hline Athlete 28 & Control & 160 & 160 & 160 & 0.00 \\
\hline
\end{tabular}

From Table 21, we can state that the mean difference between the groups consists of two components: average causal effect and the selection bias (Angrist \& Pischke, 2015) (equation 28)

$$
\begin{aligned}
\text { mean }_{\text {difference }} & =\text { Average causal effect }+ \text { Selection bias } \\
\text { Average causal ef fect } & =\frac{1}{N_{\text {Basketball }}} \sum_{i=1}^{n}\left(\text { Height }_{1 i}-\text { Height }_{0 i}\right) \\
\text { Selection bias } & =\frac{1}{N_{\text {Basketball }}} \sum_{i=1}^{n} \text { Height }_{0 i}-\frac{1}{N_{\text {Control }}} \sum_{i=1}^{n} \text { Height }_{0 i}
\end{aligned}
$$

The mean group difference we have observed $(18.87 \mathrm{~cm})$ is due to average causal effect $(0.13 \mathrm{~cm})$ and selection bias $(18.73 \mathrm{~cm})$. In other words, observed mean group difference can be explained solely by selection bias. Since we know the DGP behind the basketball data, we know that there is no systematic causal effect of playing basketball on height.

On top of the selection bias involved in the example above, other confounders might be involved, such as age, sex, race, experience and others, some of which can be measured and some might be unknown. These are also referred to as the third variable which confounds the causal relationship between treatment and the outcome. In the example above, all subjects from the Basketball group might be older males, whereas all the subjects from the Control group might be be younger females. 


\subsubsection{Ceteris paribus and the biases}

It is important to understand that, in order to have causal interpretation, comparisons need to be made under ceteris paribus conditions (Angrist \& Pischke, 2015), which is Latin for other things equal. In the basketball example above, we cannot make causal claim that playing basketball makes one taller, since comparison between the groups is not done in the ceteris paribus conditions due to the selection bias involved. We also know this since we know the simulated DGP behind the observed data.

Causal inference thus aims to achieve ceteris paribus conditions needed to make causal interpretations by careful considerations of the known and unknown biases involved (Shrier \& Platt, 2008; Angrist \& Pischke, 2015; Hernán, 2016, 2017; Rohrer, 2018; Hernán, Hsu \& Healy, 2019; Lederer et al., 2019; Hernán \& Robins).

According to Hernan et al. (Hernán, 2017; Hernán \& Robins), there three types of biases involved in causal inference: confounding, selection bias and measurement bias.

Confounding is the bias that arises when treatment and outcome share causes because treatment was not randomly assigned (Hernán, 2017; Hernán \& Robins). For example, athletes that are naturally taller might be choosing to play basketball due to success and enjoyment over their shorter peers. On the other hand, it might be some hidden confounder that motivates to-be-tall athletes to choose basketball. Known and measured confounders from the observational studies can be taken into account to create ceteris paribus conditions when estimating causal effects (Shrier \& Platt, 2008; Angrist \& Pischke, 2015; Hernán, 2017; Rohrer, 2018; Lederer et al., 2019; Hernán \& Robins).

\subsubsection{Randomization}

The first line of defence against confounding and selection bias is to randomly assign athletes to treatment, otherwise known as randomized trial or randomized experiment. Random assignment makes comparison between groups ceteris paribus providing the sample is large enough to ensure that differences in the individual characteristics such as age, sex, experience and other potential confounders are washed out (Angrist \& Pischke, 2015). In other words, random assignment works not by eliminating individual differences but rather by ensuring that the mix of the individuals being compared is the same, including the ways we cannot easily measure or observe (Angrist \& Pischke, 2015).

In case the individuals from the basketball example were randomly assigned, given the known causal DGP, then the mean difference between the groups would be more indicative of the causal effect of playing basketball on height (Table 22).

Table 22. Randomized participants 


\begin{tabular}{llr}
\hline Athlete & Treatment & Height $(\mathrm{cm})$ \\
\hline Athlete 13 & Basketball & 210 \\
Athlete 19 & Basketball & 209 \\
Athlete 09 & Basketball & 207 \\
Athlete 15 & Basketball & 205 \\
Athlete 17 & Basketball & 198 \\
Athlete 26 & Basketball & 196 \\
Athlete 12 & Basketball & 195 \\
Athlete 07 & Basketball & 192 \\
Athlete 03 & Basketball & 187 \\
Athlete 14 & Basketball & 184 \\
Athlete 10 & Basketball & 184 \\
Athlete 08 & Basketball & 182 \\
Athlete 06 & Basketball & 177 \\
Athlete 16 & Basketball & 174 \\
Athlete 28 & Basketball & 160 \\
Athlete 11 & Control & 213 \\
Athlete 01 & Control & 210 \\
Athlete 27 & Control & 208 \\
Athlete 05 & Control & 206 \\
Athlete 20 & Control & 204 \\
Athlete 25 & Control & 200 \\
Athlete 29 & Control & 191 \\
Athlete 21 & Control & 187 \\
Athlete 24 & Control & 186 \\
Athlete 18 & Control & 185 \\
Athlete 23 & Control & 184 \\
Athlete 30 & Control & 181 \\
Athlete 04 & Control & 177 \\
Athlete 22 & Control & 170 \\
Athlete 02 & Control & 168 \\
\hline & &
\end{tabular}

If we calculate the mean differences in this randomly assigned basketball treatment (Table 23), we can quickly notice that random assignment washed out selection bias involved with the observational study, and that the mean difference is closer to the known systematic causal effect. The difference between estimated systematic causal effect using mean group difference from the randomized trial is due to the sampling error which will be explained in the "Statistical Inference" section of this paper.

Table 23. Descriptive summary of randomized participants

\begin{tabular}{lrr}
\hline & Mean $(\mathrm{cm})$ & SD $(\mathrm{cm})$ \\
\hline Basketball & 190.75 & 14.23 \\
Control & 191.34 & 14.76 \\
Difference & -0.59 & 20.50 \\
\hline
\end{tabular}

Apart from creating ceteris paribus conditions, randomization generates a good control group that serves as a proxy to reveal what might have happened to the treated group in the counterfactual world where they are not treated, since Height $t_{0}$ is not known for the basketball group. Creating those conditions with randomized trial demands careful considerations and balance checking since biases can crawl inside the causal interpretation. The logic of randomized trial is simple, yet the logistics can be quite complex. For example, a 
sample of sufficient size might not be practically feasible, and disbalances in the known confounders can be still found in the groups, thus demanding further control and adjustment in the analysis (e.g. using ANCOVA instead of ANOVA, adjusting for confounders in the linear regression by introducing them as interactions and main effects) in order to create ceteris paribus conditions needed to evaluate causal claims. Belief effect can sneak in, if the treatment group knows knows they being treated, or researchers may motivate treatment groups harder, since they expect and hope for better outcomes. For this reason, blinding both the subjects and researches can be considered, as well as providing placebo to the Control group.

\subsubsection{Subject matter knowledge}

One of the main problems with randomized trials is that it cannot be done in most real life settings, either due to the ethical or practical reasons. For example, if studying effects of smoking on baby mortality and birth defects, which parent would accept being in the treatment group. Or if studying effects of resistance training on injury risk in football players, which professional organization would allow random assignment to the treatment that is lesser than the known best practices and can predispose athletes to the injuries or sub-par preparation?

For this reason, reliance on observation studies is the best we can do. However, in order to create ceteris paribus conditions necessary to minimize bias in the causal interpretations, expert subject-matter knowledge is needed, not only to describe the causal structure of the system under study, but also to specify the causal question and identify relevant data sources (Hernán, Hsu \& Healy, 2019). Imagine asking the following causal question: "Does training load lead to overuse injuries in professional sports". It takes expert subject matter knowledge to specify the treatment construct (i.e. "training load"), how is it measured, as well as the measurement error which can induce measurement bias, over which time period, as well as to specify the outcome construct (i.e. "overuse-injuries") and define variables and constructs that confound and define the causal network underlying such a question. This subject matter is fallible of course, and the constructs, variables and the causal network can be represented with pluralistic models that represents "Small World" maps of the complex "Large World", in which we are hoping to deploy the findings. Drawing assumption underlying causal structure using direct acyclical graphs (DAGs) represents a step forward in acknowledging the issues above, by providing transparency of the assumptions involved and bridging the subjective - objective dichotomy.

\subsubsection{Example of randomized control trial}

Let's consider the following example. We are interested in estimating causal effects of the plyometric training on the vertical jump height. To estimate causal effects, randomized control trial (RCT) is utilized. RCT utilizes two groups: Treatment $(\mathrm{N}=15)$ and Control $(\mathrm{N}=15)$, measured two times: Pre and Post. Treatment group received plyometric training over the course of three months, while Control group continued with normal training. The results of RCT study can be found in the Table 24. To estimate practical significance of the treatment effect, SESOI of $\pm 2.5 \mathrm{~cm}$ is selected to indicate minimal change of the practical value. The question we are trying to answer is as follows: "Does plyometric training added to normal training improves vertical jump height over period of three months?". It is important to have "well defined interventions" (Hernán \& Taubman, 2008; Hernán, 2016, 2018).

Table 24. Randomized control trial data 


\begin{tabular}{llrrr}
\hline Athlete & Group & Pre $(\mathrm{cm})$ & Post $(\mathrm{cm})$ & Change $(\mathrm{cm})$ \\
\hline Athlete 24 & Control & 45.52 & 48.28 & 2.77 \\
Athlete 20 & Control & 47.04 & 48.47 & 1.43 \\
Athlete 06 & Control & 44.03 & 44.91 & 0.88 \\
Athlete 18 & Control & 44.61 & 45.39 & 0.78 \\
Athlete 30 & Control & 40.28 & 40.63 & 0.35 \\
Athlete 08 & Control & 50.17 & 50.51 & 0.34 \\
Athlete 04 & Control & 38.07 & 38.15 & 0.08 \\
Athlete 14 & Control & 50.40 & 50.43 & 0.03 \\
Athlete 02 & Control & 52.72 & 52.62 & -0.09 \\
Athlete 12 & Control & 55.38 & 54.81 & -0.57 \\
Athlete 22 & Control & 52.81 & 51.53 & -1.28 \\
Athlete 16 & Control & 40.68 & 39.37 & -1.31 \\
Athlete 28 & Control & 34.10 & 32.39 & -1.71 \\
Athlete 10 & Control & 44.73 & 42.96 & -1.77 \\
Athlete 26 & Control & 48.91 & 46.89 & -2.02 \\
Athlete 29 & Treatment & 44.86 & 59.38 & 14.52 \\
Athlete 21 & Treatment & 44.35 & 56.62 & 12.26 \\
Athlete 15 & Treatment & 52.42 & 64.39 & 11.97 \\
Athlete 23 & Treatment & 42.71 & 52.76 & 10.05 \\
Athlete 13 & Treatment & 35.10 & 44.36 & 9.27 \\
Athlete 27 & Treatment & 43.58 & 51.87 & 8.29 \\
Athlete 07 & Treatment & 44.68 & 52.17 & 7.49 \\
Athlete 01 & Treatment & 45.95 & 52.05 & 6.10 \\
Athlete 19 & Treatment & 50.75 & 56.84 & 6.09 \\
Athlete 03 & Treatment & 46.15 & 52.14 & 5.99 \\
Athlete 17 & Treatment & 45.72 & 51.41 & 5.69 \\
Athlete 05 & Treatment & 39.66 & 44.97 & 5.30 \\
Athlete 09 & Treatment & 39.47 & 42.55 & 3.07 \\
Athlete 11 & Treatment & 40.69 & 41.06 & 0.37 \\
Athlete 25 & Treatment & 44.86 & 44.46 & -0.40 \\
\hline & & & &
\end{tabular}

Descriptive summary statistics for Treatment and Control group are enlisted in the Table 25, and visually depicted in the Figure 19 (panels A and B).

Table 25. RCT summary using mean \pm SD

\begin{tabular}{llll}
\hline Group & Pre $(\mathrm{cm})$ & Post $(\mathrm{cm})$ & Change $(\mathrm{cm})$ \\
\hline Treatment & $44.06 \pm 4.32$ & $51.14 \pm 6.63$ & $7.07 \pm 4.2$ \\
Control & $45.96 \pm 5.97$ & $45.82 \pm 6.18$ & $-0.14 \pm 1.33$ \\
\hline
\end{tabular}

Further analysis might involve separate dependent groups analysis for both Treatment and Control (Table 26), or in other words the analysis of the change scores. To estimate Cohen's d, pooled SD of the Pre- scores in both Treatment and Control is utilized.

Table 26. Descriptive analysis of the change scores for Treatment and Control groups independently 


\begin{tabular}{|c|c|c|c|c|c|c|c|c|c|c|}
\hline Group & Mean change $(\mathrm{cm})$ & SDchange $(\mathrm{cm})$ & SDpre pooled $(\mathrm{cm})$ & Cohen's d & SESOI $(\mathrm{cm})$ & Change to SESOI & SDchange to SESOI & Lower & Trivial & Higher \\
\hline Treatment & 7.07 & 4.20 & 5.21 & 1.36 & \pm 2.5 & 1.41 & 0.84 & 0.01 & 0.13 & 0.86 \\
\hline Control & -0.14 & 1.33 & 5.21 & -0.03 & \pm 2.5 & -0.03 & 0.27 & 0.04 & 0.94 & 0.02 \\
\hline
\end{tabular}

But we are not that interested in independent analysis of Treatment and Control group, but rather in their differences and understanding the causal effects of the treatment (i.e. understanding and estimating parameters of the underlying DGP). As stated, treatment effect consists of two components: systematic component or fixed effect (i.e. average causal effect), and stochastic component or random effect (i.e. that varies between individuals). As already explained, Control group serves as a proxy to what might have happened to the Treatment group in the counterfactual world, and thus allows to interpret treatment effects causally. There are two effects at play with this RCT: treatment effect and non-treatment effect. The latter captures all effects not directly controlled by a treatment, but assumes it affects both groups equally. For example, if we are treating kids for longer period of time, non-treatment effect might be related to the growth and associated effects. Another non-treatment effect is measurement error (discussed in more details in "Classical Test Theory" section).

The following equation captures the essence of estimating treatment effects from Pre and Post scores in the Treatment and Control groups (equation 29):

$$
\begin{aligned}
\text { Treatment }_{\text {post }} & =\text { Treatment }_{\text {pre }}+\text { Treatment }_{\text {Effect }}+\text { NonTreatment Effect } \\
\text { Control }_{\text {post }} & =\text { Control }_{\text {pre }}+\text { NonTreatment Effect } \\
\text { NonTreatment Effect } & =\text { Control }_{\text {post }}-\text { Control }_{\text {pre }} \\
\text { Treatment Effect } & =\text { Treatment }_{\text {post }}-\text { Treatment }_{\text {pre }}-\text { NonTreatment Effect } \\
\text { Treatment Effect } & =\left(\text { Treatment }_{\text {post }}-\text { Treatment }_{\text {pre }}\right)-\left(\text { Control }_{\text {post }}-\text { Control }_{\text {pre }}\right) \\
\text { Treatment Effect } & =\text { Treatment }_{\text {change }}-\text { Control }_{\text {change }}
\end{aligned}
$$

From the above equation, the differences between the changes in Treatment and Control groups can be interpreted as the estimate of the causal effect of the treatment. Table 27 contains descriptive statistics of the change score differences. Panel $\mathrm{C}$ in the Figure 19 depicts distribution of the change scores and reflect the calculus in the Table 27 graphically.

Table 27. Descriptive statistics of the change score differences

\begin{tabular}{rrrrrr}
\hline Mean difference $(\mathrm{cm})$ & Cohen's d & Difference to SESOI & Lower diff & Trivial diff & Higher diff \\
\hline 7.21 & 5.41 & 1.44 & -0.03 & -0.81 & 0.84 \\
\hline
\end{tabular}



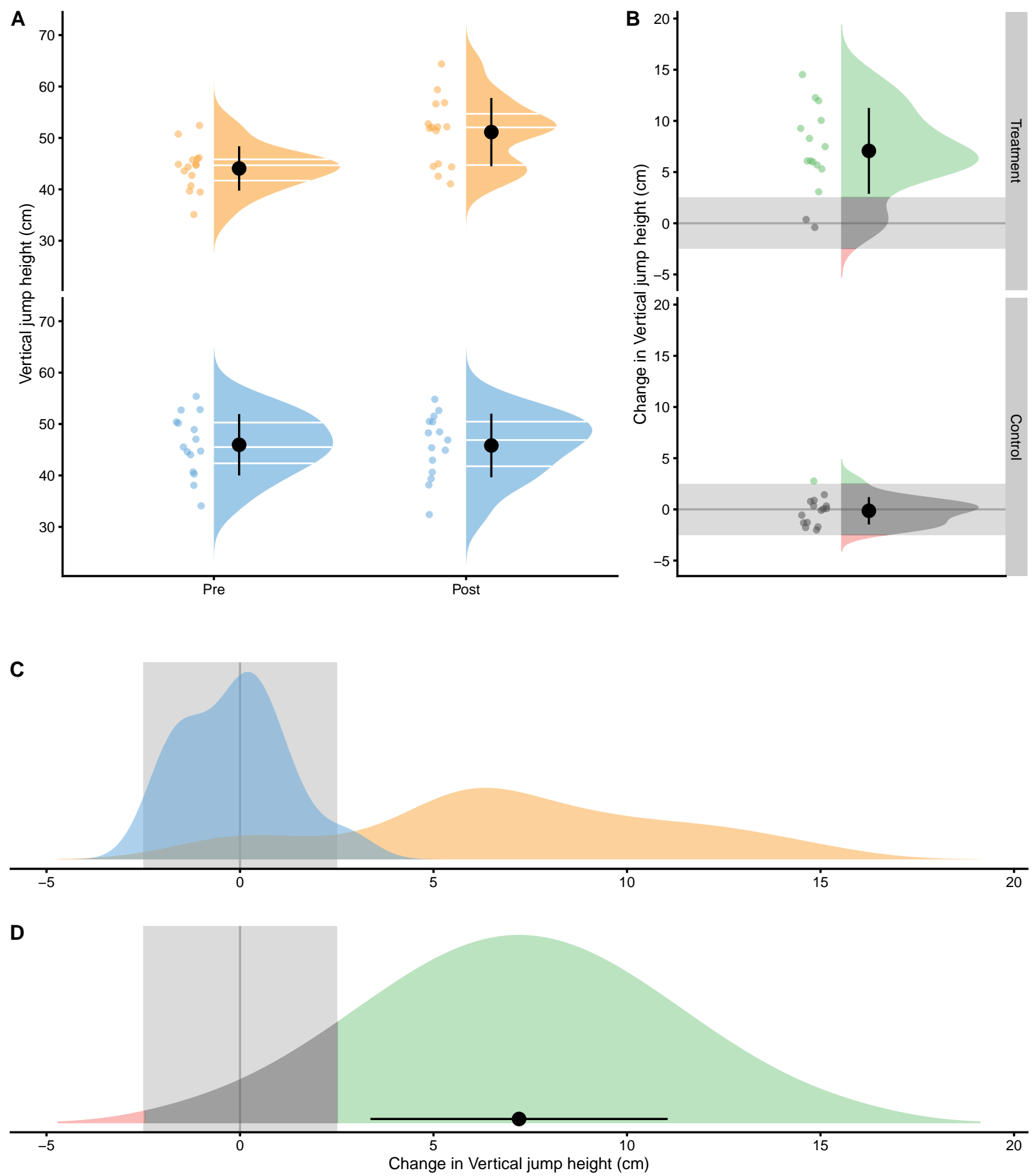

Figure 19. Visual analysis of RCT using Treatment and Control groups. A. Raincloud plot of the Pre-test and Post-test scores for Treatment and Control groups. Blue color indicates Control group and orange color indicates Treatment group across all panels. B. Raincloud plot of the change scores for the Treatment and Control groups. SESOI is indicated with a grey band. C. Density plot of Treatment and Control group change scores. SESOI is indicated with a grey band. D. Graphical representation of the causal Treatment effect. Green area indicates proportion of higher than SESOI treatment effects, red indicates proportion of negative and lower than SESOI treatment effects, and grey indicates treatment effects that are within SESOI. Please refer to the main text for explanation 
Cohen's d in the Table 27 is calculated by using the following equation (30) and it estimates standardized difference between change scores in Treatment and the Control groups:

$$
\text { Cohen's } d=\frac{\text { mean }_{\text {treatment group change }}-\text { mean }_{\text {control group change }}}{S D_{\text {control group change }}}
$$

Besides estimating systematic components of the treatment (i.e. the difference between the mean change in Treatment and Control group), we might be interested in estimating random component and proportions of lower, trivial and higher effects compared to SESOI. Unfortunately, differences in lower, trivial, and higher proportions from Table 27 don't answer this question, but rather the expected difference in proportions compared to Control (e.g. the expected improvement of 0.84 in observing proportion of higher change outcomes compare to Control).

Since the changes in Treatment group are due both to the treatment and non-treatment effects (equation 31), the average treatment effect (systematic component) represents the difference between the mean changes in Treatment and Control group (Table 28). In the same manner, the variance of the change scores in the Treatment group are due to the random component of the treatment and non-treatment effects. Assuming normal distribution of the random components, the $S D$ of the treatment effects $\left(S D_{T E}\right)^{18}$ is estimated using the following equation (31):

$$
\begin{aligned}
\epsilon_{\text {treatment group change }} & =\epsilon_{\text {treatment effect }}+\epsilon_{\text {nontreatment effect }} \\
\epsilon_{\text {control group change }} & =\epsilon_{\text {nontreatment effect }} \\
\epsilon_{\text {treatment effect }} & =\epsilon_{\text {treatment group change }}-\epsilon_{\text {control group change }} \\
\epsilon_{\text {treatment effect }} & \sim \mathcal{N}\left(0, S D_{T E}\right) \\
\epsilon_{\text {nontreatment effect }} & \sim \mathcal{N}\left(0, S D_{N T E}\right) \\
\epsilon_{\text {treatment group change }} & \sim \mathcal{N}\left(0, S D_{\text {treatment group change }}\right) \\
\epsilon_{\text {control group change }} & \sim \mathcal{N}\left(0, S D_{\text {control group change }}\right) \\
S D_{T E} & =\sqrt{S D_{\text {treatment group change }}^{2}-S D_{\text {control group change }}^{2}}
\end{aligned}
$$

Thus, the estimated parameters of the causal treatment effects in the underlying DGP are the following ones (equation 32). This treatment effect is graphically depicted in the Figure 19 (panel D).

$$
\begin{aligned}
\text { Treatment effect } & \sim \mathcal{N}\left(\text { Mean }_{T E}, S D_{T E}\right) \\
\text { Mean }_{T E} & =\text { Mean } \text { treatment group change }- \text { Mean } \text { control group change } \\
S D_{T E} & =\sqrt{S D_{\text {treatment group change }}^{2}-S D_{\text {control group change }}^{2}}
\end{aligned}
$$

Using SESOI, one can also estimate the proportion of lower, trivial and higher changes (responses) caused by treatment. The estimates of the causal treatment effects, with accompanying proportions of responses are enlisted in the Table 28.

Table 28. Estimates of the causal treatment effects

\footnotetext{
${ }^{18}$ Also referred to as $S D_{I R}$ or standard deviation of the intervention responses (Hopkins, 2015b; Swinton et al., 2018).
} 


\begin{tabular}{|c|c|c|c|c|c|c|c|}
\hline Average causal effect $(\mathrm{cm})$ & Random effect $(\mathrm{cm})$ & SESOI $(\mathrm{cm})$ & Average causal effect to SESOI & SESOI to random effect & Lower & Trivial & Higher \\
\hline 7.21 & 3.98 & \pm 2.5 & 1.44 & 1.26 & 0.01 & 0.11 & 0.88 \\
\hline
\end{tabular}

Therefore, we can conclude that plyometric training over three months period on top of the normal training cause improvements in vertical jump height (in the sample collected; generalizations beyond sample are discussed in the "Statistical inference" section). The expected improvement (i.e. average causal effect or systematic effect) is equal to $7.21 \mathrm{~cm}$, with 1,11 , and $88 \%$ of athletes having lower, trivial and higher improvements.

\subsubsection{Prediction as a complement to causal inference}

Although the ausal analysis above is needed to understand the underlying DGP, particularly the effects of the intervention, if we try to predict changes in vertical jump height using "Pre" and "Group" 19 variables, the prediction error would be too big for practical use. Table 29 contains cross-validated predictions using vertical jump change or post treatment performance as target variables, while using "Group" and "Pre" variables as predictors. ${ }^{20}$

Table 29. Predictive performance of the linear model predicting Change and Post-test variables

\begin{tabular}{llrrrrrr}
\hline Target & SESOI $(\mathrm{cm})$ & cvRMSE $(\mathrm{cm})$ & SESOI to cvRMSE & cvEquivalence & RMSE $(\mathrm{cm})$ & SESOI to RMSE & Equivalence \\
\hline Change & \pm 2.5 & 3.21 & 1.56 & 0.55 & 2.98 & 1.68 & 0.59 \\
Post & \pm 2.5 & 3.33 & 1.50 & 0.54 & 2.98 & 1.68 & 0.59 \\
\hline
\end{tabular}

This indicates that high explanatory power doesn't automatically yield high predictive power (Shmueli, 2010). The selection of statistical analysis is thus related to the question asked. In my opinion, it would be insightful to complement causal estimates with prediction estimates. With the example above, we can predict the direction of the effect (using expected systematic change of $7.21 \mathrm{~cm}$ and proportions of 1,11 , and $88 \%$ for lower, trivial and higher change magnitudes), but we are unable to predict individual changes within acceptable practical precision (using SESOI as an anchor). In other words, we know that the effect will be $88 \%$ beneficial (i.e. higher than SESOI), but we are not able to predict individual reactions. More about these individual vs. group estimates is discussed in the later section.

In the example above, we have assumed binary treatment (either plyometric training is done or not), whereas in real life there can be nuances, particularly in volume of jumps performed, making the treatment continuous rather than binary variable. This way, we are interested in the effects of number of jumps on the changes in vertical jump height.

There could also be hidden variables involved that moderate and mediate the effects of the treatment ${ }^{21}$. For example, the higher someone jumps in the Pre-test, the lower the change in the Post-test (i.e. it is harder to improve vertical jump height). Or, the stronger someone is in the Pre-test (measured using relative back squat 1RM) the more potentiated the effects of the plyometrics are. All these are needed expert subject-matter knowledge, required to understand the underlying DGP. With such causal structure, we do not have direct treatment effect (plyometric $\rightarrow$ change in vertical jump) only anymore, but moderated and mediated, or

\footnotetext{
${ }^{19}$ Group variable is dummy coded to 0 for Control and 1 for Treatment.

${ }^{20}$ Both "Group" and "Pre" are used to indicate all available information before treatment is utilized.

${ }^{21}$ Using the randomization in the RCT it is assumed that these hidden variables are equally distributed, and that there is no selection bias involved.
} 
indirect effects estimated using the interactions in the regression models. These topics are beyond the scope of this paper, particularly the overview nature of these sections.

Interpreting and understanding direct and indirect effects can be quite difficult, especially when causal structure becomes complex. Visualization techniques such as already mentioned PDP and ICE graphs can be utilized to understand the causal mechanism (Goldstein et al., 2013; Zhao \& Hastie, 2019). These techniques can also be implemented in observational studies similar to DAGs, but with considerable domain knowledge and assumptions needed (Zhao \& Hastie, 2019). Although predictive analysis, particularly those using black box machine learning models, has been criticized to lack causal interpretation (Pearl \& Mackenzie, 2018; Hernán, Hsu \& Healy, 2019; Pearl, 2019), they can complement causal (or explanatory) analysis (Breiman, 2001; Shmueli, 2010; Yarkoni \& Westfall, 2017).

Let's expand the previous RCT to include Pre-test relative back squat 1RM variable (Strength; "Squat 1RM" on table) (Table 30).

Table 30. Randomized control trial data, but now with omitted variable: Squat 1RM

\begin{tabular}{lrlrrr}
\hline Athlete & Squat 1RM & Group & Pre $(\mathrm{cm})$ & Post $(\mathrm{cm})$ & Change $(\mathrm{cm})$ \\
\hline Athlete 24 & 2.13 & Control & 45.52 & 48.28 & 2.77 \\
Athlete 20 & 1.79 & Control & 47.04 & 48.47 & 1.43 \\
Athlete 06 & 2.22 & Control & 44.03 & 44.91 & 0.88 \\
Athlete 18 & 1.17 & Control & 44.61 & 45.39 & 0.78 \\
Athlete 30 & 1.64 & Control & 40.28 & 40.63 & 0.35 \\
Athlete 08 & 2.01 & Control & 50.17 & 50.51 & 0.34 \\
Athlete 04 & 1.15 & Control & 38.07 & 38.15 & 0.08 \\
Athlete 14 & 1.66 & Control & 50.40 & 50.43 & 0.03 \\
Athlete 02 & 1.40 & Control & 52.72 & 52.62 & -0.09 \\
Athlete 12 & 1.59 & Control & 55.38 & 54.81 & -0.57 \\
Athlete 22 & 0.98 & Control & 52.81 & 51.53 & -1.28 \\
Athlete 16 & 1.23 & Control & 40.68 & 39.37 & -1.31 \\
Athlete 28 & 1.40 & Control & 34.10 & 32.39 & -1.71 \\
Athlete 10 & 1.01 & Control & 44.73 & 42.96 & -1.77 \\
Athlete 26 & 1.22 & Control & 48.91 & 46.89 & -2.02 \\
Athlete 29 & 1.95 & Treatment & 44.86 & 59.38 & 14.52 \\
Athlete 21 & 1.56 & Treatment & 44.35 & 56.62 & 12.26 \\
Athlete 15 & 1.81 & Treatment & 52.42 & 64.39 & 11.97 \\
Athlete 23 & 1.48 & Treatment & 42.71 & 52.76 & 10.05 \\
Athlete 13 & 1.41 & Treatment & 35.10 & 44.36 & 9.27 \\
Athlete 27 & 1.65 & Treatment & 43.58 & 51.87 & 8.29 \\
Athlete 07 & 1.43 & Treatment & 44.68 & 52.17 & 7.49 \\
Athlete 01 & 1.30 & Treatment & 45.95 & 52.05 & 6.10 \\
Athlete 19 & 1.55 & Treatment & 50.75 & 56.84 & 6.09 \\
Athlete 03 & 1.04 & Treatment & 46.15 & 52.14 & 5.99 \\
Athlete 17 & 1.37 & Treatment & 45.72 & 51.41 & 5.69 \\
Athlete 05 & 1.11 & Treatment & 39.66 & 44.97 & 5.30 \\
Athlete 09 & 0.99 & Treatment & 39.47 & 42.55 & 3.07 \\
Athlete 11 & 0.85 & Treatment & 40.69 & 41.06 & 0.37 \\
Athlete 25 & 0.59 & Treatment & 44.86 & 44.46 & -0.40 \\
\hline & & & & & \\
\hline
\end{tabular}

Using linear regression model and "Change" as the target variable with "Group", "Pre" and "Strength" variables as predictors, as well as interactions "Group:Pre" and "Group:Strength", the following regression 
parameters are estimated $^{22}$ (Table 31$)$.

Table 31. Linear regression with interaction parameter estimates

\begin{tabular}{lr}
\hline & Estimate \\
\hline (Intercept) & -3.63 \\
GroupTreatment & 1.65 \\
Pre & 0.00 \\
Strength & 2.41 \\
GroupTreatment:Pre & -0.13 \\
GroupTreatment:Strength & 8.61 \\
\hline
\end{tabular}

Explaining how these estimates are interpreted is beyond the scope of this paper since this model involves both mediation and moderation effects of changes in vertical jump height. Apart from linear regression, it is important to estimate predictive performance of this model. Table 32 contains predictive performance of this model using cross-validations for both Change and Post-test as target variables.

Table 32. Predictive performance of linear regression model with interactions for both Change and Post-test as target variables

\begin{tabular}{|c|c|c|c|c|c|c|c|}
\hline Target & SESOI $(\mathrm{cm})$ & cvRMSE (cm) & SESOI to cvRMSE & cvEquivalence & $\operatorname{RMSE}(\mathrm{cm})$ & SESOI to RMSE & Equivalence \\
\hline Change & \pm 2.5 & 1.59 & 3.15 & 0.86 & 1.25 & 4.01 & 0.95 \\
\hline Post & \pm 2.5 & 1.67 & 3.00 & 0.85 & 1.25 & 4.01 & 0.95 \\
\hline
\end{tabular}

As can be seen in the Table (32), linear model with interactions produces almost perfect predictions in both Change and Post-test vertical jump height. This means that the variance in the treatment effect is now explained with additional variables and their interactions ${ }^{23}$.

We are not only interested in prediction, but rather in the underlying causal structure and explaining this model performance when we intervene on variables (i.e. what happens to the target variable when I change $\mathrm{X}$ from $a$ to $b$, while keeping other variables fixed - which is ceteris paribus condition). For this reason, PDP and ICE plots can be used to give causal interpretation of the model. I will get back to PDP and ICE plots in "Counterfactual prediction" section.

The main critique of Judea Pearl regarding the use of predictive models and techniques is the lack of counterfactual causal interpretation (Pearl \& Mackenzie, 2018; Pearl, 2019), particularly with observational studies. I agree that "the role of expert knowledge is the key difference between prediction and causal inference tasks" (Hernán, Hsu \& Healy, 2019) p.44 and that "both prediction and causal inference require expert knowledge to formulate the scientific question, but only causal inference requires causal expert knowledge

\footnotetext{
${ }^{22}$ This model definition refers to our pre-existing beliefs around causal structure underlying intervention. If the statistical analysis is done to confirm our structural causal model, then this represents confirmatory analysis. Usually these studies need to be pre-registered with the exact analysis workflow and assumption defined before data is collected. This is required because in the exploratory analysis we can play with the data, try different models and select one that fits the data best. Exploratory analysis is useful for generating models and hypothesis for future studies, but also introduces hindsight bias since the model is selected after seeing the data or the results of multiple analyses. Very related bias is called p-harking (Hypothesizing After Results are Known) which involves modifying the data or the analysis, after seeing the results, to reach statistical significance (discussed later). In predictive analysis this hind-sight bias is reduced by using hold-out sample and cross-validation and evaluating the final model performance on the data that has not been seen by the model.

${ }^{23}$ If you remember the footnote discussion on aleatory and epistemic uncertainty, this is example where what we believed to be aleatory uncertainty (inter-individual error or variation that we could not predict) was actually epistemic uncertainty that we reduced with omitted variable(s) (1RM Strength and interaction terms). Although the inter-individual variation in the treatment effect remains $\left(S D_{T E}\right.$ or $\left.S D_{I R}\right)$, we are now able to predict individual responses more precisely.
} 
to answer the question" (Hernán, Hsu \& Healy, 2019) p.45, but this doesn't negate the need for providing predictive performance, as well as helping in interpreting the model when such data is available (Zhao \& Hastie, 2019).

According to Zhao and Hastie (Zhao \& Hastie, 2019), p.1: "There are three requirements to make causal interpretations: a model with good predictive performance, some domain knowledge in the form of a causal diagram and suitable visualization tools.". The common denominator among multiple experts is that for causal inference and causal interpretation there is a need for domain knowledge, particularly when RCTs are not available. This domain knowledge can be made more transparent by using DAGs and other structural diagrams, and thus help in falsifying assumptions (Hernan, 2002; Hernán, 2017; Gelman \& Hennig, 2017; Hernán, Hsu \& Healy, 2019). Adding prediction to explanatory models can be seen as complement, particularly since statistical analysis has been neglecting predictive analysis over explanatory analysis (Breiman, 2001; Shmueli, 2010; Yarkoni \& Westfall, 2017).

\subsubsection{Responders vs non-responders}

One particular use of the predictive analysis is in the identification of responders and non-responders to the treatment (Hecksteden et al., 2015, 2018; Hopkins, 2015b; Swinton et al., 2018). Common approach used in sport science (Hopkins, 2004b), that I will name observed outcome approach (further discussed in "Measurement Error" section), uses known SESOI and measurement error to estimate probability of lower, trivial, and higher changes. Using this approach, we can classify athletes with high probability of higher change score as responders, those with high probability of trivial change score as non-responders, and finally those with high probability of lower change score as negative-responders. This approach is useful in figuring out who responded positively or negatively to a particular treatment, but it doesn't take into account information that might help explain the response (for example someone missing treatment session or having lower or higher treatment dose).

Another approach, that I have termed residuals approach or model-based approach can be used to help identifying outlier to intervention.

This approach uses observed change and model predicted change to indicate individuals who changed more or less than predicted by the model. If this difference between observed change and predicted change (or residual) is bigger than SESOI, this individual is flagged. Figure 20 depicts such analysis using the above linear regression with interactions model for both Change and Post-Test prediction. 
A

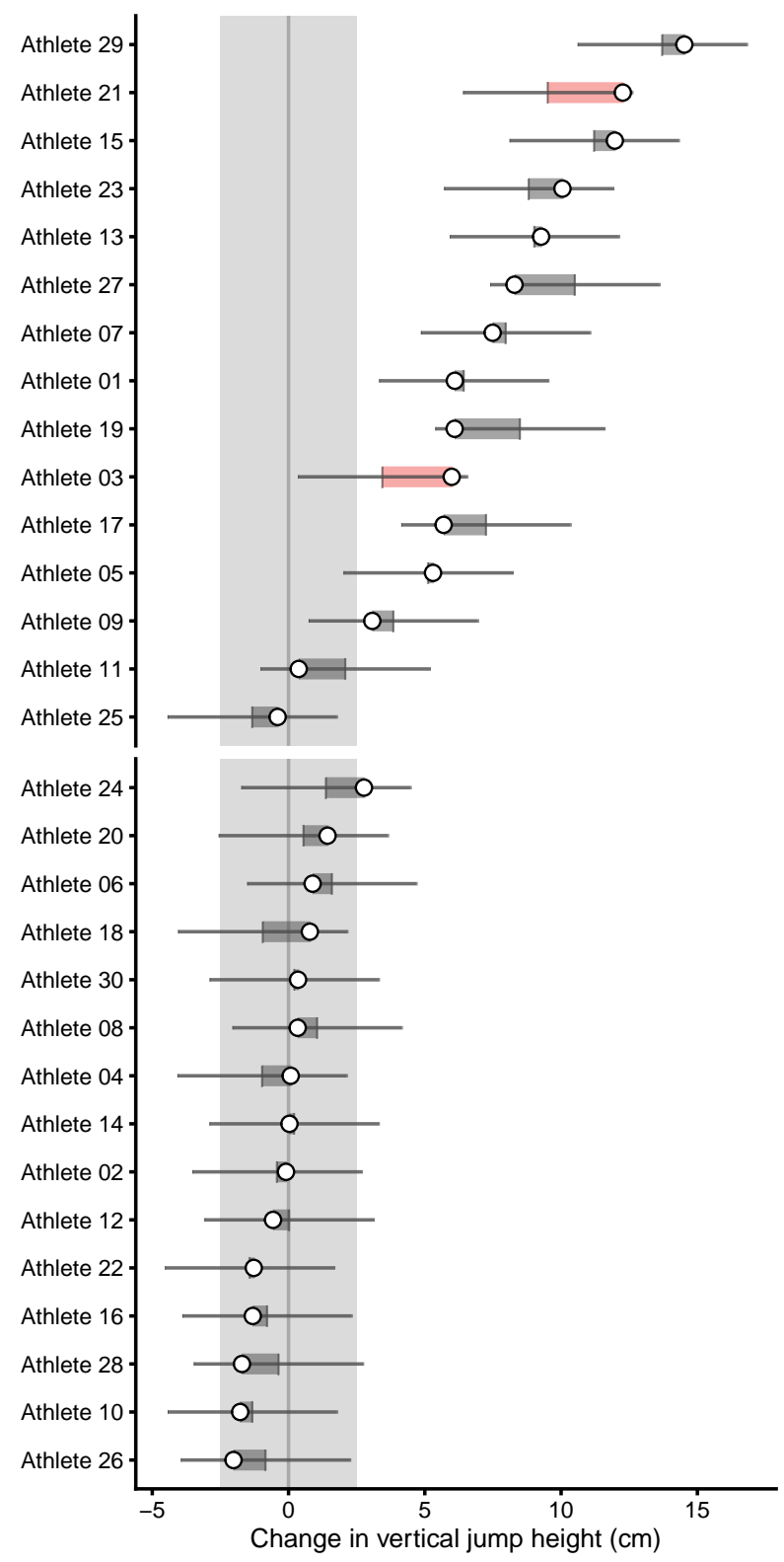

B

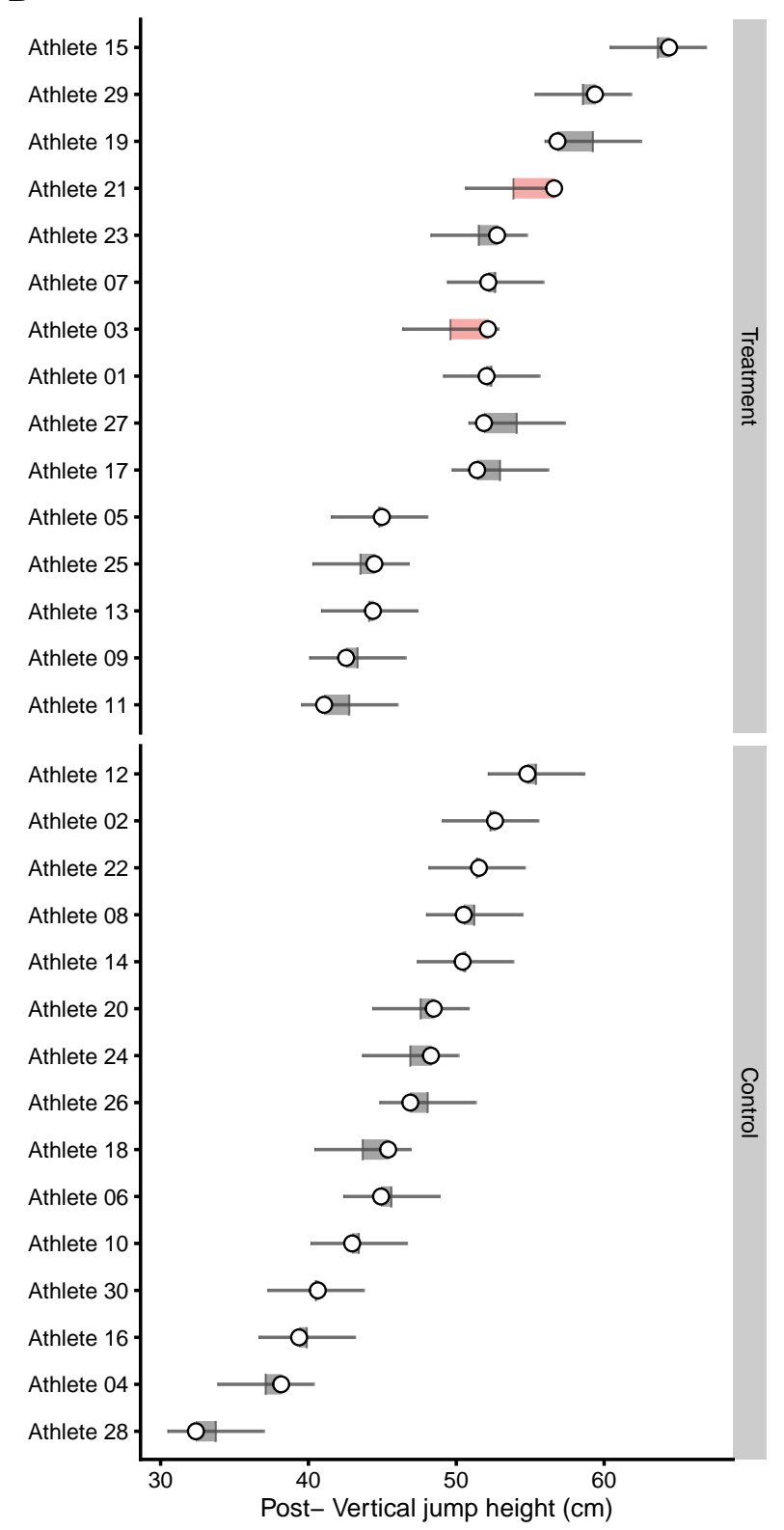

Figure 20. Model-based approach in identifying treatment outliers using linear regression with interactions as predictive model. A. Individual change scores for Treatment and Control groups. Observed change score is depicted with the white dot. Horizontal error bars represent model prediction for a particular individual (vertical line) with accompanying $95 \%$ prediction intervals calculated using $1.96 \times c v R M S E$. The difference, or the residual, between observed and predicted change is indicated with the horizontal bar. If this residual is bigger than SESOI, it is colored in red. This indicates that the individual model prediction is practically different than observed score. In other words, expected change based on the model and the data is practically different than observed and might need further inspection.

B. The same analysis as the one used in panel A, but now for Post-test variable

In the Figure 20 athletes who showed lower or higher change compared to what was predicted by the model are flagged with red color. As opposed to the observed outcome approach, a model-based prediction approach uses ceteris paribus in estimating responders vs. non-responders, or at least providing residual for such a 
decision. For example, everything else being equal, based on predictor variables, the expected change is higher or lower than the observed change. This indicates that there might be something not-identified by predictive algorithm and thus needs to be flagged for a further analysis. In the image above, Athletes 21 and 03 are flagged because, based on the information available to the model (and the model itself), he or she should have manifested higher or lower observed changed. This residual (predicted score - observed score) can be divided by cvRMSE to get standardized residual (equation 33). This standardized residual can be used to sort athletes and compare responses (changes) on different interventions or target metrics that are not on the same scales.

$$
\text { Standardized Residual }_{i}=\frac{\text { Change }_{i}-\text { Change }_{i}}{\text { cvRMSE }}
$$

\subsubsection{Counterfactual predictions}

As explained, causal inference and explanatory modelling are interested in understanding, or at least quantifying the causal effects. This is done by elaborate and transparent control of the confounders and study designs. Predictive modelling on the other hand is interested in providing valid predictions on new or unseen data without assuming underlying DGP, or treating it as a black-box. In certain scenarios, when confounders are controlled, predictive modelling can be interpreted causally (Zhao \& Hastie, 2019). With observational studies, there is always a risk of not controlling all important confounders, but transparency of the causal model is there to be falsified and discussed openly (Hernan, 2002; Hernán \& Taubman, 2008; Hernán, 2016, 2018; Gelman \& Hennig, 2017; Hernán, Hsu \& Healy, 2019). Even with the RCTs, there might be uncertainties in applying the findings from the experiments to realistic stings ((Heckman, 2005; Gelman, 2011)).

PDP and ICE plots are model-agnostic tools for interpreting black-box predictions that can be used in causal interpretations only after the effort to define the causal structure with domain-specific expertise is taken into account. But let's take this approach in estimation, based on the predictive model, counterfactual change and Post-test scores when athletes do not receive treatment (for the Treatment group), or when they receive treatment (for the Control group). This way we are predicting potential outcomes.

How is this done? We simply switch Group variable, as well as interaction terms and see how the model predicts for each athlete. This is exactly the same strategy used in the PDP and ICE plots. These counterfactual predictions are depicted in the Figure 21. 
A

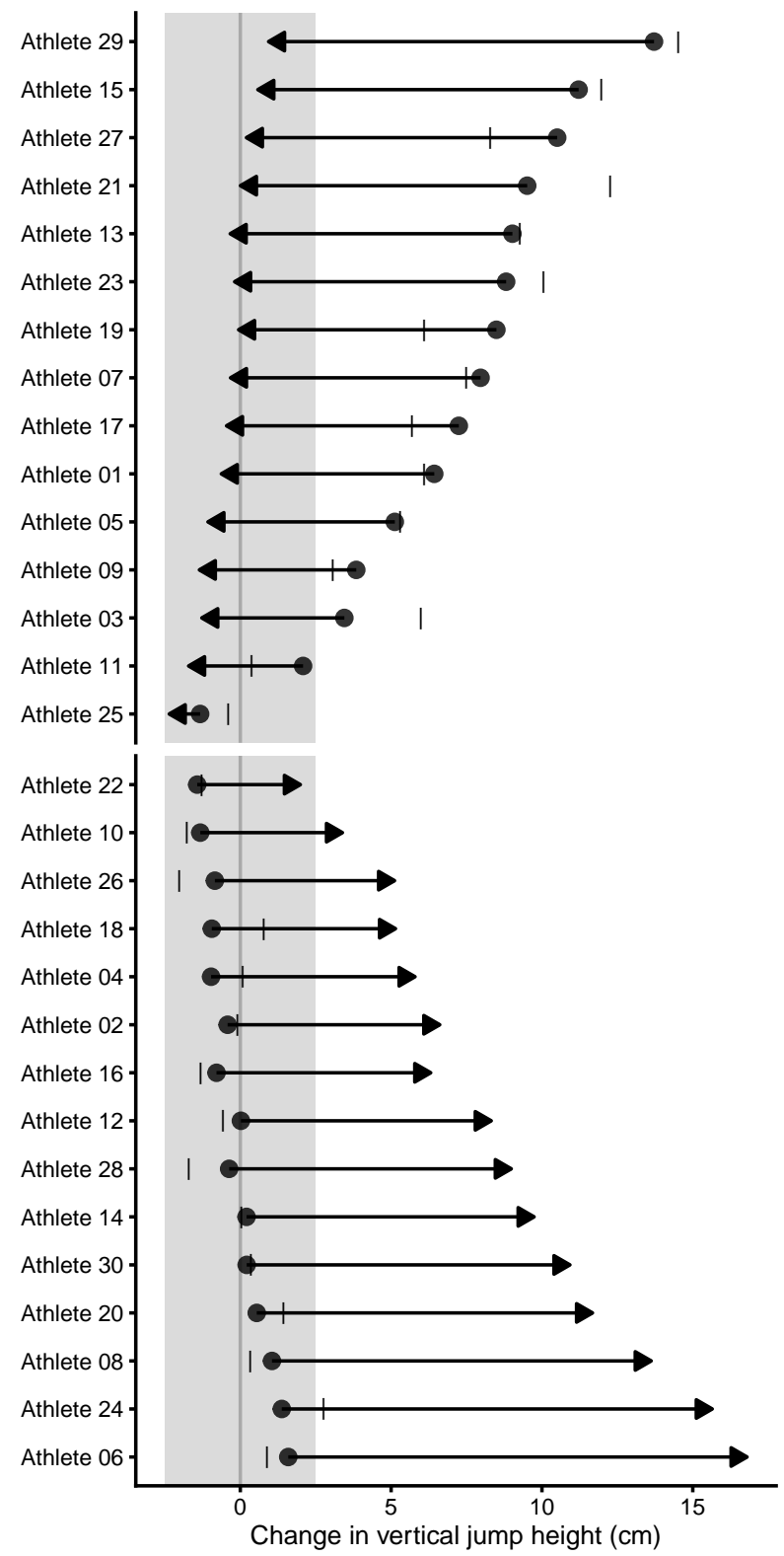

B

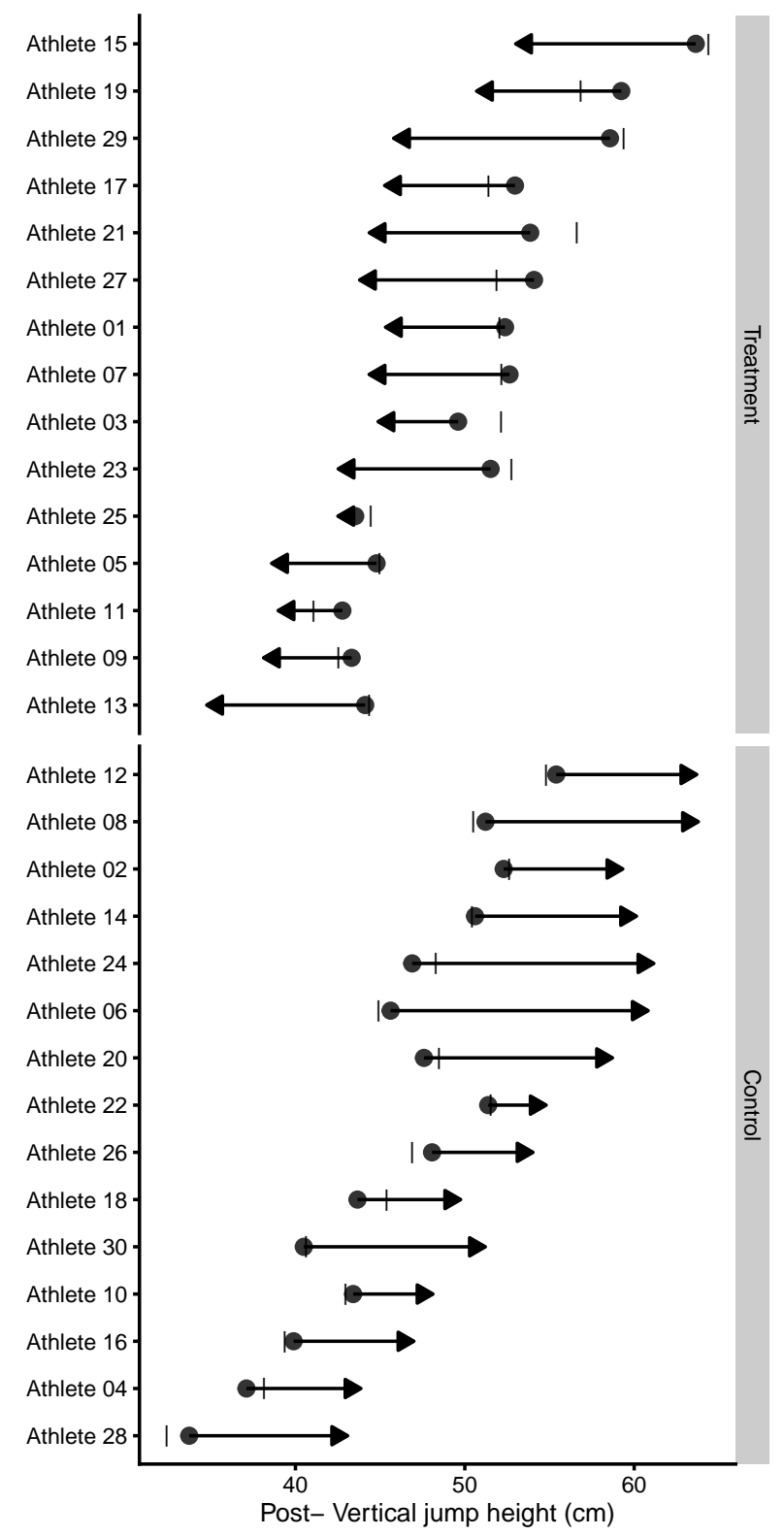

Figure 21. Counterfactual individual responses. A. Black dot indicates predicted scores, while vertical lines indicates observed score. Directional arrow is estimated by changing the Group variable and predicting how each athlete wouls respond in counterfactual way, while everything else being equal (but with updated interaction variables, since they involve Group variable as well). This helps in answering counterfactual question of how an individual athlete would respond if he or she didn't receive or did receive the treatment. B. The same rationale and visualization, but for Post-test variable

Counterfactual predictions can be used to identify potential non-responders to the treatment. This can be named counterfactual approach. In other words, given the model, we are interested in how would the athlete respond in the counterfactual world.

Table 33 contains average predicted counterfactual effects, as well their SDs for both Control and Treatment groups using Change and Post-Test as target variables. 
Table 33. Average predicted counterfactual effects

\begin{tabular}{llrrrrr}
\hline Group & Target & Mean Change $(\mathrm{cm})$ & SD Change $(\mathrm{cm})$ & Lower & Trivial & Higher \\
\hline Treatment & Post-Test & -7.61 & 3.02 & 0.95 & 0.04 & 0.00 \\
Control & Post-Test & 8.81 & 3.45 & 0.00 & 0.03 & 0.97 \\
Treatment & Change & -7.61 & 3.02 & 0.95 & 0.04 & 0.00 \\
Control & Change & 8.81 & 3.45 & 0.00 & 0.03 & 0.97 \\
\hline
\end{tabular}

But what if we are interested in the effect of changing some pre-test variable, for example strength? The causal question might be "How would athlete respond to the treatment if his strength was higher or lower?". This is estimated using PDP and ICE plot techniques, where all variables except the one of interest are kept the same (ceteris paribus) whereas the variable of interest is changed. Then the model predictions for the target variable are depicted. Since we know the correlated variables (i.e. subject matter knowledge ), we can make valid counter-factual graph. We are interested in potential-outcomes of Change and Post-Test if the pre-test strength was different. To put everything on the same scale, we will use factor of the Strength change, from 0.8 to 1.2 (where 1 is the true Strength level for a particular individual). The counter factual predictions are depicted in the Figure 22. 
A

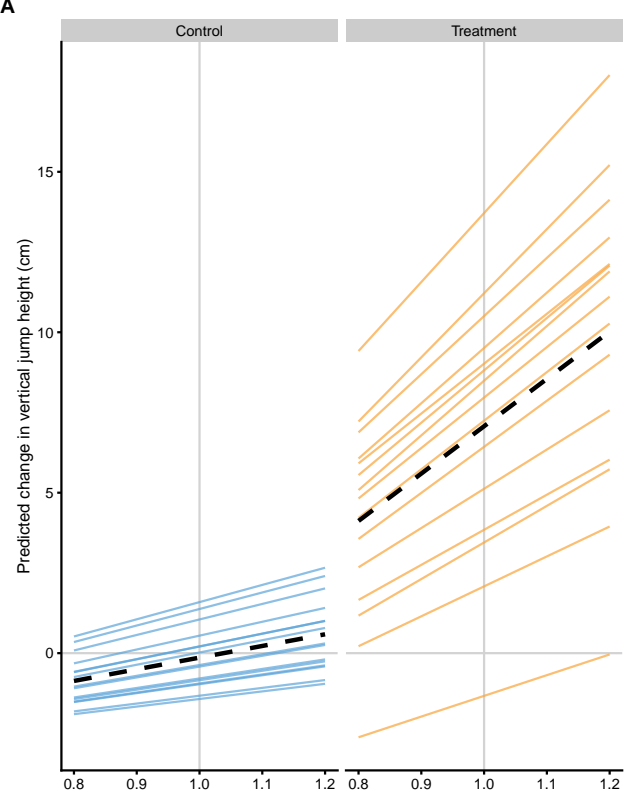

c

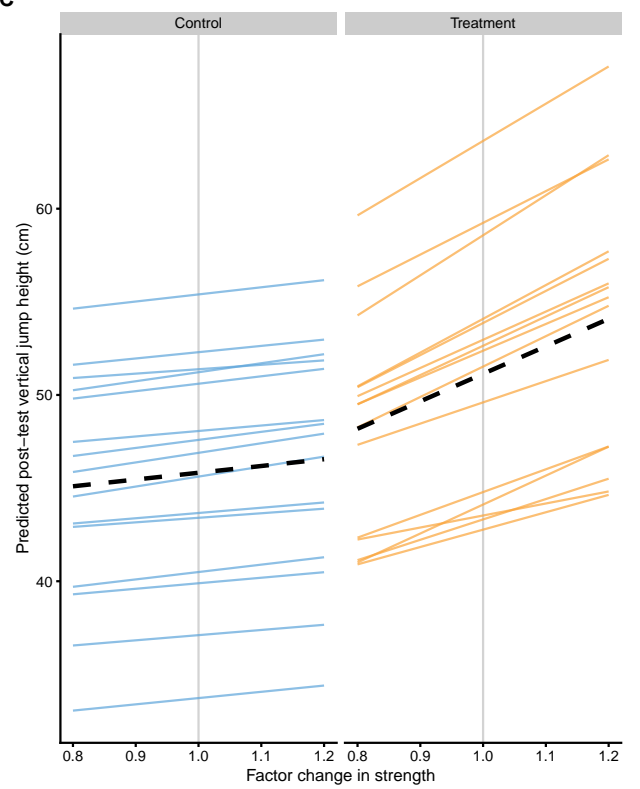

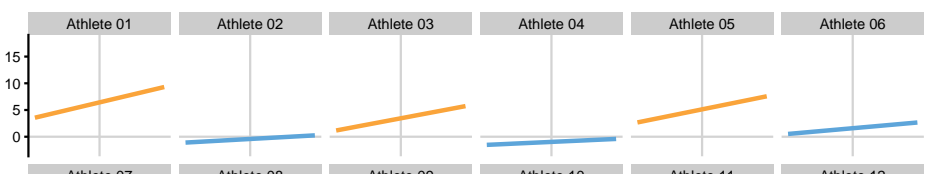
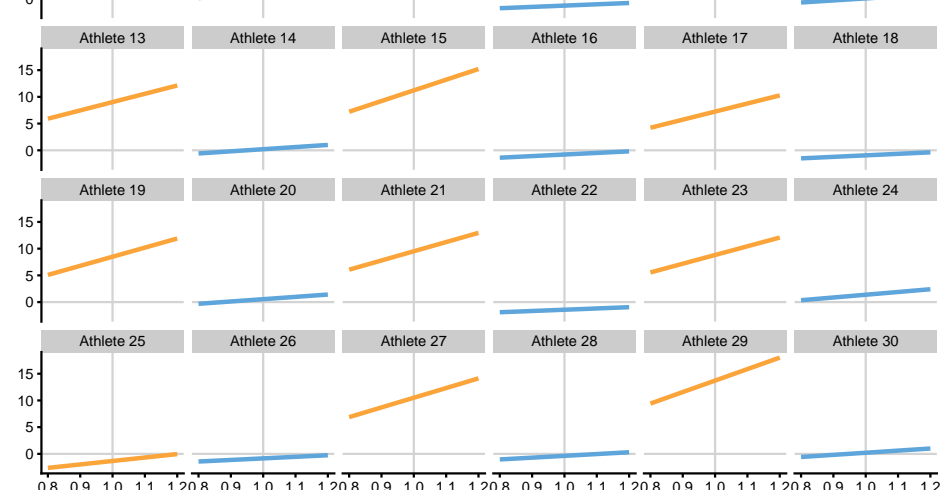

D

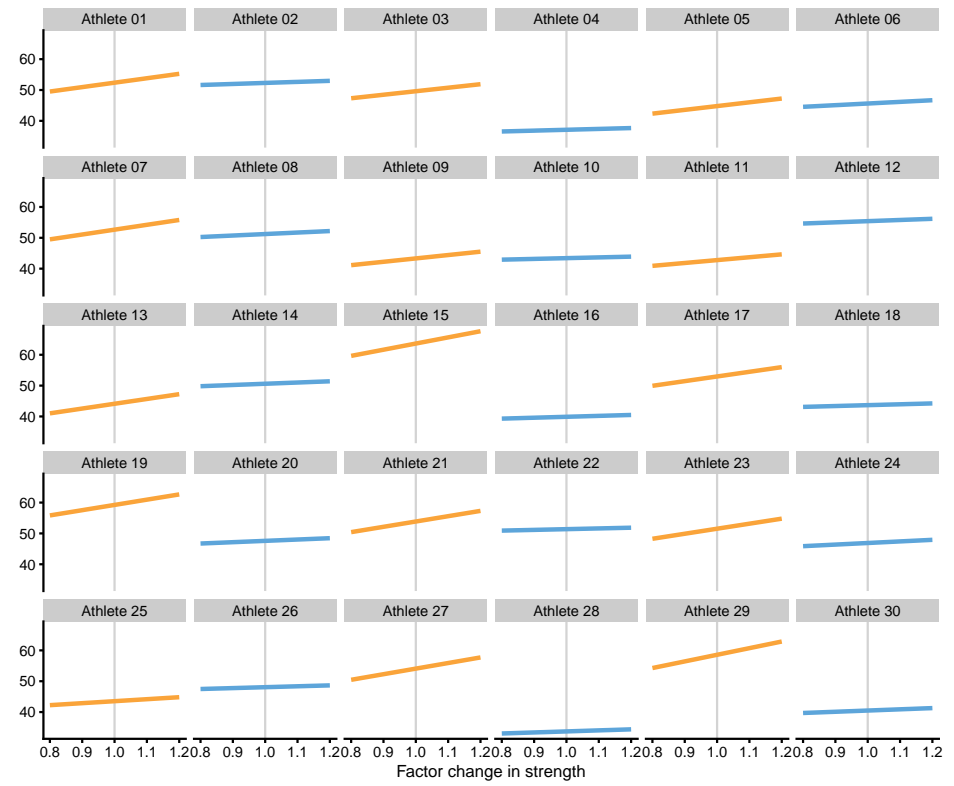

Figure 22. Counter factual effect of Strength variable changes on individual change and

Post-test score. A. PDP and ICE plot for change in vertical jump when Strength changes from 0.8 to 1.2. Analysis is separated for Control (blue color) and Treatment (orange color) groups. Thin lines represents ICE or individuals (depicted separately on the panel B), while dashed line represents the average or PDP. As can be seen, not only the Treatment produce higher change (fixed effect indicated by the gap between the lines), it also interacts with Strength (squat 1RM) indicating that stronger individuals demonstrates higher effects (indirect or interaction effect indicated by higher slope of the line compared to Control group). B. Individual prediction from panel A depicted separately. $\mathbf{C}$ and $\mathbf{D}$. Identical analysis and visualization using

Post-test variable

From Panels A and C, we can visually identify the interaction effect of Group:Strength, since the PDP lines (the average lines) for each group are not parallel. Although these PDP and ICE plots can be interpreted causally in their RCT example, we have committed one major assumption - the assumption of ergodicity 


\subsubsection{Ergodicity}

Ergodic process is underlying DGP that is equivalent at between-individual (or inter-individual; or group-based analysis) and within-individual (or intra-individual or simply individual-based analysis) levels. Thus the causal effects identified using between-individual analysis can be applied to understand within-individual causation as well. Non-Ergodic process on the other hand differs between these two levels and effects identified at between-individual level cannot be inferred to within individual level.

Few authors have already brought into question the generalizability of group-based research to individualbased prediction and causal inferences (Molenaar, 2004; Molenaar \& Campbell, 2009; Fisher, Medaglia \& Jeronimus, 2018; Glazier \& Mehdizadeh, 2018). Here is an interesting quote from Molenaar (Molenaar \& Campbell, 2009):

"Most research methodology in the behavioral sciences employs inter-individual analyses, which provide information about the state of affairs of the population. However, as shown by classical mathematical-statistical theorems (the ergodic theorems), such analyses do not provide information for, and cannot be applied at, the level of the individual, except on rare occasions when the processes of interest meet certain stringent conditions. When psychological processes violate these conditions, the inter-individual analyses that are now standardly applied have to be replaced by analysis of intra-individual variation in order to obtain valid results." p. 112

The individual counterfactual predictions above thus rely on ergodicity, which represents big assumption. This means that we should be cautious when generalizing model predictions and causal explanations from group-based level to individual-level.

Data analysis at the individual level (i.e. collecting multiple data point for individuals) and identifying individual causal effects and predictions might be the step forward, but even with such an approach we are retrodicting under ceteris paribus conditions and we might not be able to predict future responses. For example, if we have collected responses to various interventions for a particular individual, we might not be able to estimate with certainty how he or she is going to respond to a familiar treatment in the future, since such a prediction relies on stationarity of the parameters or the underlying DGP (Molenaar \& Campbell, 2009).

But this doesn't imply that all our efforts are useless. We just need to accept the uncertainty and the assumptions involved. For example, for completely novel subject, the best response or prediction estimate is the expected response calculated by using the group-based analysis (i.e. average treatment effect). This represents the most likely response or prediction. But on top of providing these distribution-based or groupbased estimates, one can provide expected uncertainties showing individual-based or response proportions as anchor-based (magnitude-based) estimates (Norman et al., 2001; Estrada, Ferrer \& Pardo, 2019). It is important to note that these usually estimate the same information (Norman et al., 2001; Estrada, Ferrer \& Pardo, 2019); e.g. the higher the Cohen's d the higher the proportion of higher responses. Thus reporting magnitude-based proportions as uncertainties together with expected responses using average-based approach at least help in communicating uncertainties much better than reporting solely average-based estimates. When warranted with the research question, researchers should also report predictive performances on unseen data, as well as underlying assumption using graphical models such as DAGs.

It might be the best to conclude the section on causal inference with the quote from Andwer Gelmans paper (Gelman, 2011):

"Casual inference will always be a challenge, partly because our psychological intuitions do not always match how the world works. We like to think of simple causal stories, but in the social world, causes and effects are complex. We - in the scientific community - still have not come to any agreement on how best to form consensus on causal questions: there is a general acceptance that experimentation is a gold standard, but it is not at clear how much experimentation should be done, to what extent we can trust inference from observational data, and to what extent experimentation should be more fully incorporated into daily practice (as suggested by some in the "evidence-based medicine" movement)." (p. 965) 


\section{Statistical Inference}

Why did estimates diverge from the true parameters in the above examples? Why did estimated treatment effect differed from the true treatment effect we used to simulate the data? This brings us to the important concept in statistical analysis: a difference between the true parameter in the population and the sample estimate. Population refers to all members of specified group, wheras sample contains only a part, or a subset of a population from which it is taken. To generate the data in simulations, we have assumed DGP in the population, out of which a single sample was drawn.

The mathematical notation to differentiate true population parameters and sample estimates, or statistics involve using Greek letters to represent true population parameters, and Latin letters to represent sample estimates. For example, $\mu$ stands for true population mean, while $\bar{x}$ stand for sample mean, where bar symbol ( $(")$ indicates mean. When it comes to standard deviation, $\sigma$ stands for true population parameters, while $S D$ stands for sample estimate. Sometimes the hat symbol ("'s) is used to denote estimator. For example, $\hat{T E}$ would be a sample estimate of the true $T E$ in the population.

The difference between the true population parameter and the sample estimate is due to the sampling error, which will be covered shortly. In the simulations, we are certain about the true population parameters, but in real life we are uncertain about the true parameters and we need to somehow quantify this uncertainty. This is the objective of statistical inference. In other words, with statistical inference we want to generalize from sample to a population, while taking into account uncertainties of the estimates.

\subsection{Two kinds of uncertainty, two kinds of probability, two kinds of statistical inference}

In the RCT example, using the Pre-Test score and Group variable we have estimated predictive performance of the model using Change as the target variable. In other words, uncertainty around individual effects were practically useless. This uncertainty decreased as we introduced new variable Strength, as well as interactions between Group and Strength, and Group and Pre-Test. With this model we have achieved much better predictive performance. This type of uncertainty can be called epistemic uncertainty (O'Hagan, 2004), which is a result of lack of knowledge or incomplete information.

In the "Prediction" section of this paper, I have utilized irreducible error in the DGP to represent stochastic component or the random error. Due to this random error, scores will differ from sample to sample. This type of uncertainty can be called aleatory uncertainty (O'Hagan, 2004), which results due randomness. Another flagship example of the aleatory uncertainty is tossing a dice, drawing a card from a shuffled pack, or random sampling that produces the sampling error.

You can argue of course that aleatory uncertainty is ultimately epistemic uncertainty. For example, if I knew more about the measurement device (e.g. power supply oscillations, air humidity changes) I would reduce the random error. Or if I knew infinite details about the dice tossing, I would be able to predict exactly the number that will be landed. Philosophers have been arguing about these issues for ages, and it is not in the domain of this paper to delve deeper into the matters of uncertainty.

The theory of statistical inference and statistics in general rests on describing uncertainties by using probability. Since there are two kinds of uncertainty, there are two kinds of probabilities and their meaning. Aleatory uncertainties, like tossing a dice or random sampling, are described using long-frequency definition of probability. For example, it can happen that I toss six for 4 times in a row, but in the long-run, which means infinite number of times, probability of tossing a six is equal to $1 / 6$, or probability of 0.166 , or $16.6 \%$ (assuming a fair dice of course). Probability viewed from this perspective represents long-frequency number of occurrences of the event of interest, divided by number of total events. For example, if I toss a dice for 1000 times, and if I get number six for 170 times, the probability of tossing a six is equal to $170 / 1000$, or $17 \%$.

With epistemic uncertainty, probability of a proposition simply represents a degree-of-belief in the truth of that proposition (O'Hagan, 2004). The degree-of-belief interpretation of probability is referred to as subjective probability or personal probability, while long-frequency interpretation of probability is referred to as objective 
probability. There are two major schools of statistical inference leaning either on long-frequency interpretation of probability, called frequentist, or leaning on degree-of-belief interpretation of probability, called Bayesian. There are of course many nuances and other schools of statistical inference (Dienes, 2008; Efron \& Hastie, 2016; Gelman \& Hennig, 2017) which are beyond the scope of this paper. The additional approach to inference that will be considered in this paper as a preferred approach is the bootstrap (Hesterberg, 2015; Efron \& Hastie, 2016; Rousselet, Pernet \& Wilcox, 2019b,a). But more about it later.

To better explain the differences between the frequentist and Bayesian approach to statistical inference I am going to use known men mean height and SD in the population. The population parameter of interest is the population mean (Greek $\mu$ ) estimated using the sample mean $(\bar{x})$.

\subsection{Frequentist perspective}

Using simulations is outstanding teaching tool (Hopkins, 2007; Carsey \& Harden, 2013), very useful for understanding the frequentist inference. Figure 23 (Panels A and B) depicts hypothetical men population where mean height $\mu$ is $177.8 \mathrm{~cm}$ and SD $(\sigma)$ is $10.16 \mathrm{~cm}$. From this population we are randomly drawing $\mathrm{N}=5$ (left side panels on Figure 23) and $\mathrm{N}=20$ (right side panels on Figure 23) individuals for which we estimate the mean height. Individuals are represented as blue dots (Panels C and D on Figure 23), whereas estimated mean height is depicted as orange dot. Now imagine we repeat this sampling 50 times, calculate the mean for every sample, and then draw the distribution of the sampled means (Panels E an F on Figure 23). This distribution is called sampling distribution and the SD of this distribution is titled standard error or sampling error. Since our estimate of interest is the mean, standard deviation of the sampling distribution of the mean is called standard error of the mean (SEM). On Panels E and F, mean of the sampling means is indicated by a black dot, and error bars represent SEM. 

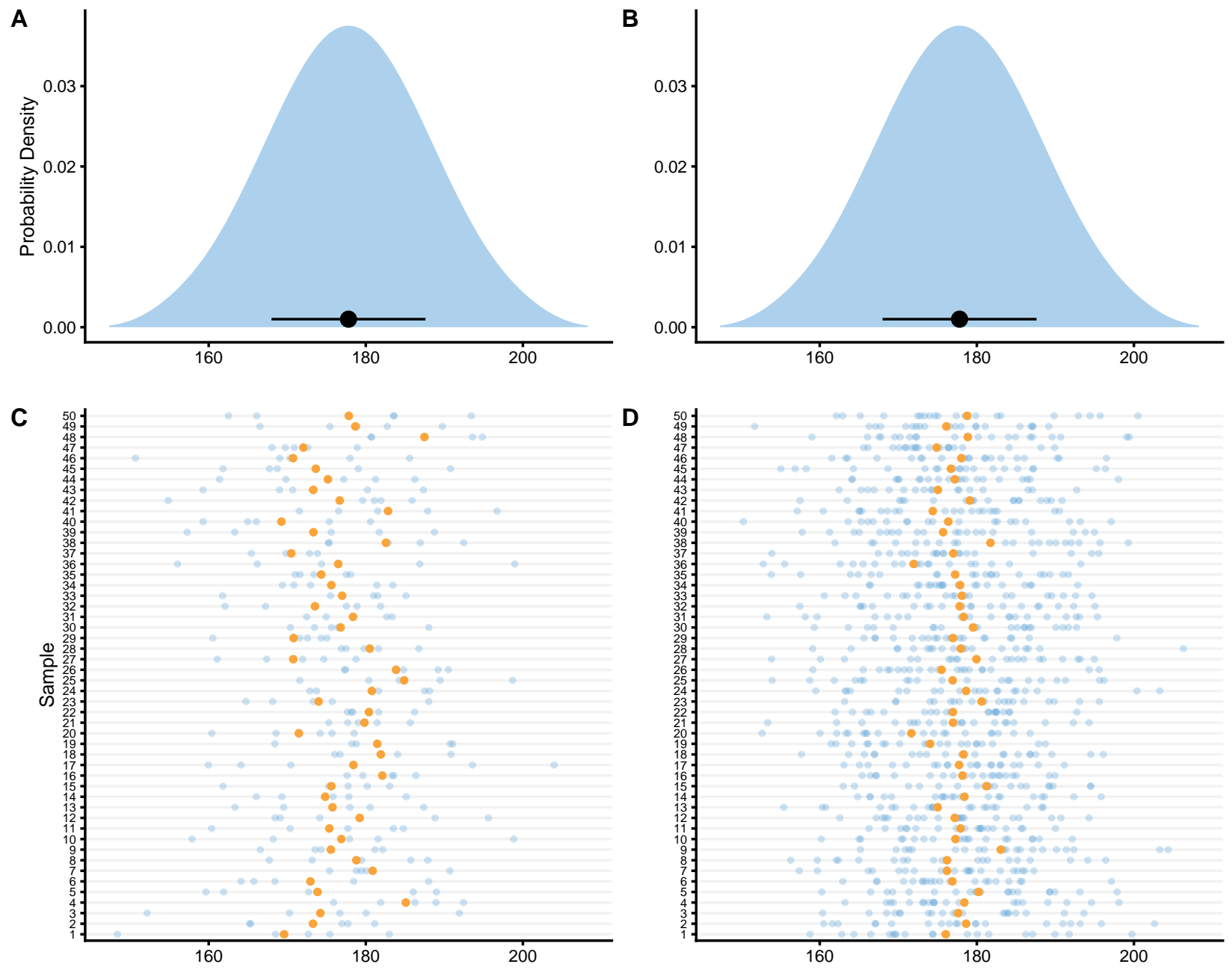

D
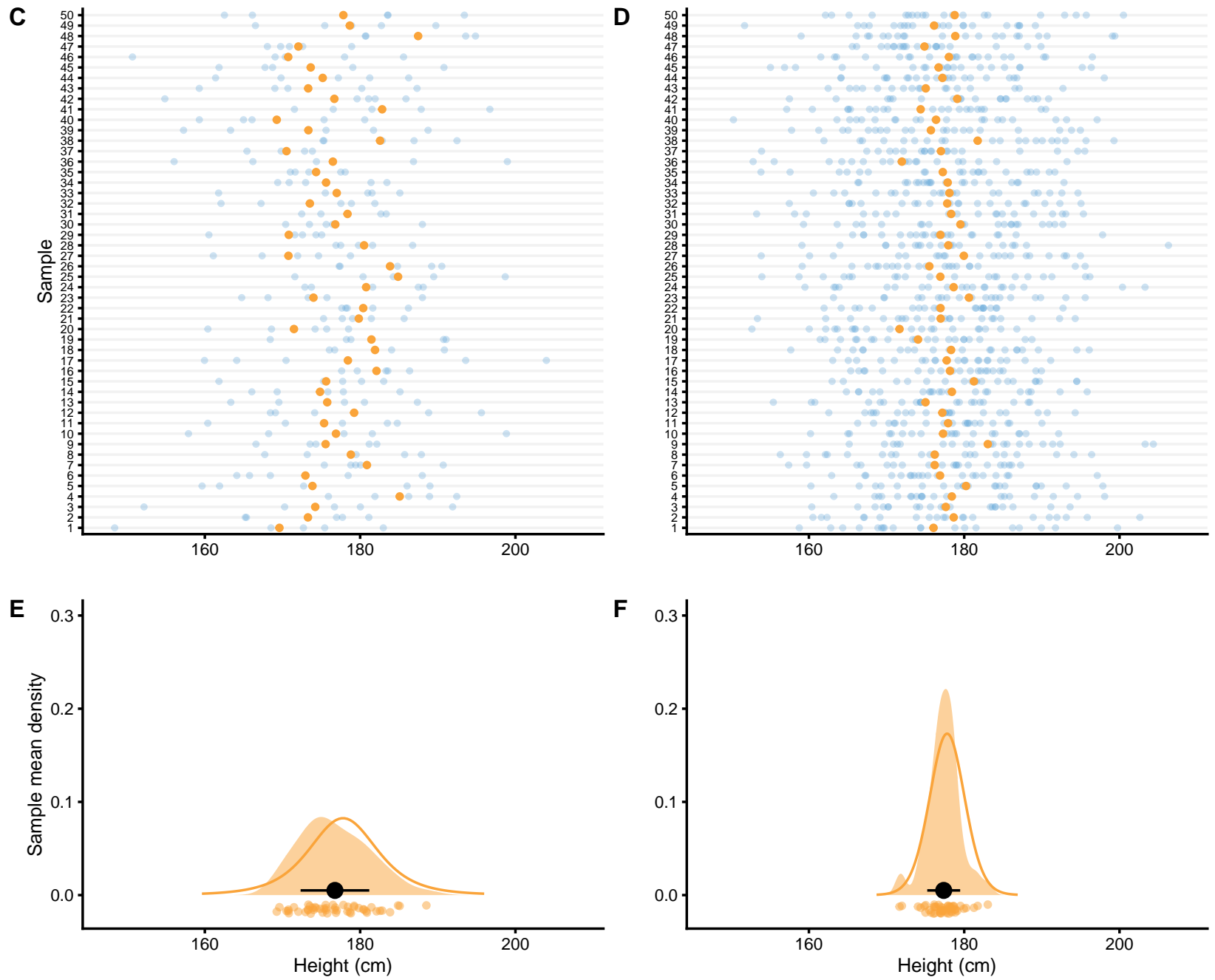

Figure 23. Sampling distribution of the mean. A and B. Distribution of the height in the population. From this population we draw samples. C and D. 50 sample are taken with $\mathrm{N}=5$ (panel C) and $\mathrm{N}=20$ (panel D) observations. Each observation is indicated by a blue dot. Calculated mean, as a parameter of interest, is indicated by an orange dot. E and F. Distribution of collected sample means from panels $\mathrm{C}$ and D. This distribution of the sample means is narrower, indicating higher precision when higher $\mathrm{N}$ is used. Black dot indicates the mean of the sample means, with error bars indicating SD of sample means. Orange line represents hypothetical distribution of the sample means when number of samples is infinitely large 
As can be seen from the Figure 23, the sampling distribution of the mean looks like normal distribution. If the number of samples reach very large number or infinity, the sampling distribution of the mean will eventually be distributed with the SEM equal to (equation 34):

$$
S E M=\frac{\sigma}{\sqrt{N}}
$$

This theoretical distribution is overlaid on the acquired sampling distribution from 50 samples in the Figure 23 (Panels E and F). Since the true $\sigma$ is not known, sample SD is utilized instead, in order to estimate the true SEM (equation 35):

$$
S \hat{E} M=\frac{S D}{\sqrt{N}}
$$

The take-home point is that the larger the sample, the smaller the standard error, which is visually seen as narrower sampling distribution (compare $\mathrm{N}=5$ and $\mathrm{N}=20$ sampling distributions on Figure 23). Another conclusion regarding frequentist inference, is that calculated probabilities revolve around this and other long-frequency sampling distributions. Everything else are details. But as the saying goes, the devil is in the detail.

Sampling distributions and equations for standard errors are derived algebraically for most estimators (e.g. mean, SD, Cohen's d), but for some it might be hard to derive them, so other solutions do exist (like bootstrapping which will be covered in designated section). For example, sampling distribution of the change score proportions can be very difficult to be derived algebraically (Swinton et al., 2018). For some estimators, mean of the long-frequency samples is different than the true population value, thus these estimators are termed biased estimators. One example of the biased estimator would be SD of the sample where we divide the variance with $N$, instead of $N-1$. Estimators that have mean of the long-frequency samples equal to the true population value are called unbiased estimators.

Although the sampling distribution of the mean looks like a normal distribution, it actually belongs to the Student's $t$ distribution, which has fatter tails for smaller samples (Figure 24). Besides mean and standard deviation, Student $\mathrm{t}$ distribution also has degrees of freedom (DF) parameters, which is equal to N-1 for the sample mean. Normal distribution is equal to Student $t$ distribution when DF is infinitely large.

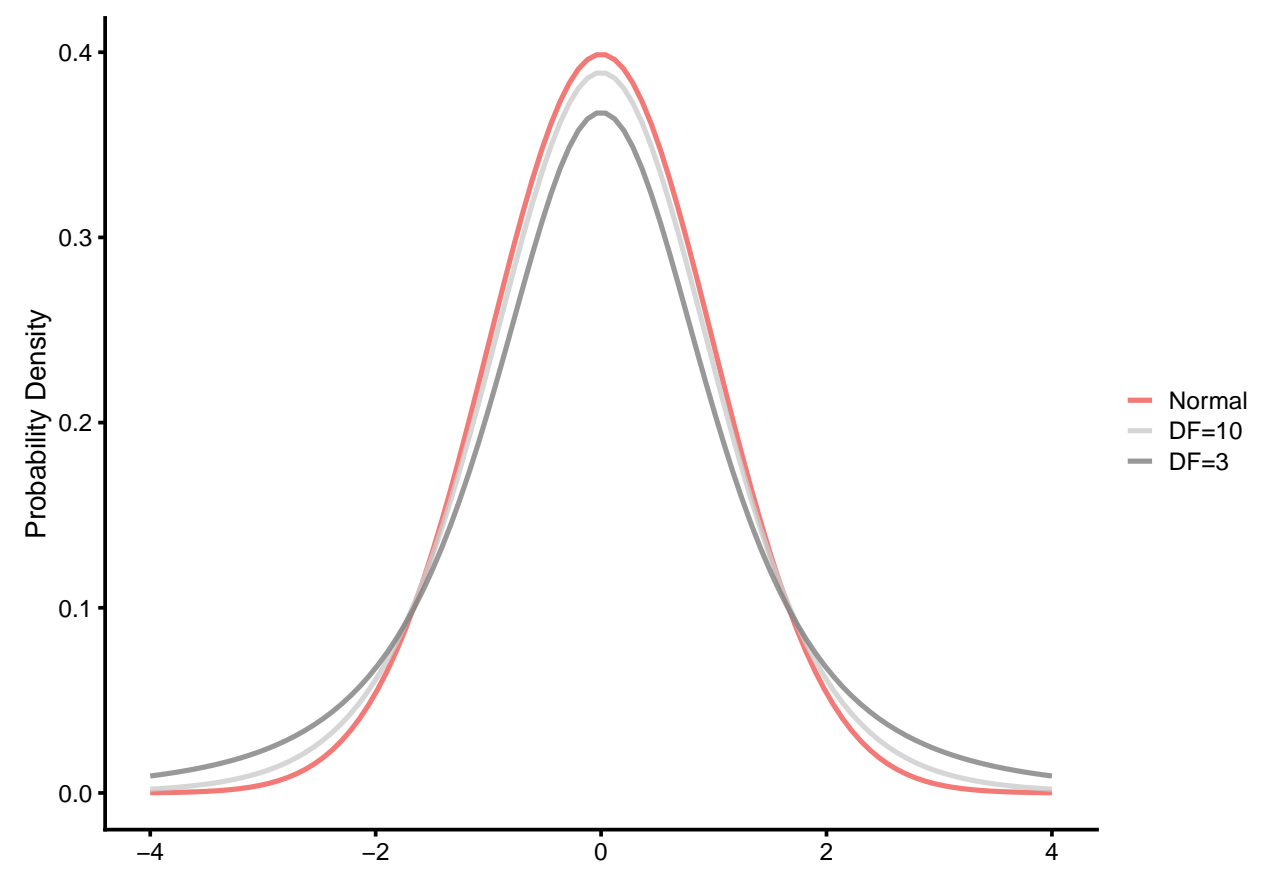




\section{Figure 24. Student's t-distribution}

Since Student's t distribution is fatter on the tails, critical values that cover 90, 95, and $99 \%$ of distribution mass are different than the commonly used ones for the normal distribution. Table 34 contains critical values for different DF. For example, $90 \%$ of the sampling distribution will be inside the $\bar{x} \pm 1.64 \times S E M$ interval for the normal distribution, but for Student $\mathrm{t}$ with $\mathrm{DF}=5,90 \%$ of the sampling distribution will be inside the $\bar{x} \pm 2.02 \times S E M$ interval.

Table 34. Critical values for Student's t distribution with different degrees of freedom

\begin{tabular}{lccccr}
\hline & $50 \%$ & $90 \%$ & $95 \%$ & $99 \%$ & $99.9 \%$ \\
\hline $\mathrm{DF}=5$ & 0.73 & 2.02 & 2.57 & 4.03 & 6.87 \\
$\mathrm{DF}=10$ & 0.70 & 1.81 & 2.23 & 3.17 & 4.59 \\
$\mathrm{DF}=20$ & 0.69 & 1.72 & 2.09 & 2.85 & 3.85 \\
$\mathrm{DF}=30$ & 0.68 & 1.70 & 2.04 & 2.75 & 3.65 \\
$\mathrm{DF}=50$ & 0.68 & 1.68 & 2.01 & 2.68 & 3.50 \\
(Normal) & 0.67 & 1.64 & 1.96 & 2.58 & 3.29 \\
\hline
\end{tabular}

\subsubsection{Null-Hypothesis Significance Testing}

There are two approaches to statistical inference, be it frequentist or Bayesian: hypothesis testing and estimation (Cumming, 2014; Kruschke \& Liddell, 2018b). I will focus on the former, although latter will be covered as well. For the frequentist inference, mathematics behind both of these are the same and both involve standard errors.

Null-hypothesis significance testing (NHST) is still one of the most dominant approaches to statistical inference, although heavily criticized (for example see (Cumming, 2014; Kruschke \& Liddell, 2018b)). In Figure 23, we have sampled from the known population, but in practice we don't usually know the true parameter values in the population, nor we are able to collect data from the whole population (unless it is a small one, but there is no need for statistical inference then, since the whole population is represented in our sample). Thus, we need to use sampled data to make inferences about the population. With NHST we want to test sample parameter (i.e. mean in this case) against the null-hypothesis $\left(H_{0}\right)$. Null-hypothesis usually takes the no effect value, but it can take any value of interest for the researcher.

Although this sounds mouthful, a simple example will make it clearer. Imagine that we do know the true population mean height, but in one particular region the mean height of the sample differs from the known population mean. If we assume that this region belongs to the same population, then we want to test to see how likely are we to sample mean we have acquired or larger one. This way we have assumed known population mean height as our null-hypothesis, and using sample SD we can estimate the sampling distribution assuming null-hypothesis as true.

Figure 25 contains known population mean height as the null-hypothesis and estimated probabilities of observing sample mean of $180,182.5$, and $185 \mathrm{~cm}$ (or $+2.2,+4.7,+7.2 \mathrm{~cm}$ difference) or larger for sample sizes $\mathrm{N}=5, \mathrm{~N}=10$ and $\mathrm{N}=20$. Panel A on Figure 25 depicts one-sided approach for estimating probability of observing these sample mean heights. One-sided approach is used when we are certain about the direction of the effect. Two-sided approach, on the other hand, calculates probability for the effect of the unknown direction. In this example that would be sample mean height difference of $\pm 2.2, \pm 4.7, \pm 7.2 \mathrm{~cm}$ or larger. Two-sided approach is depicted on the Panel B (Figure 25). 


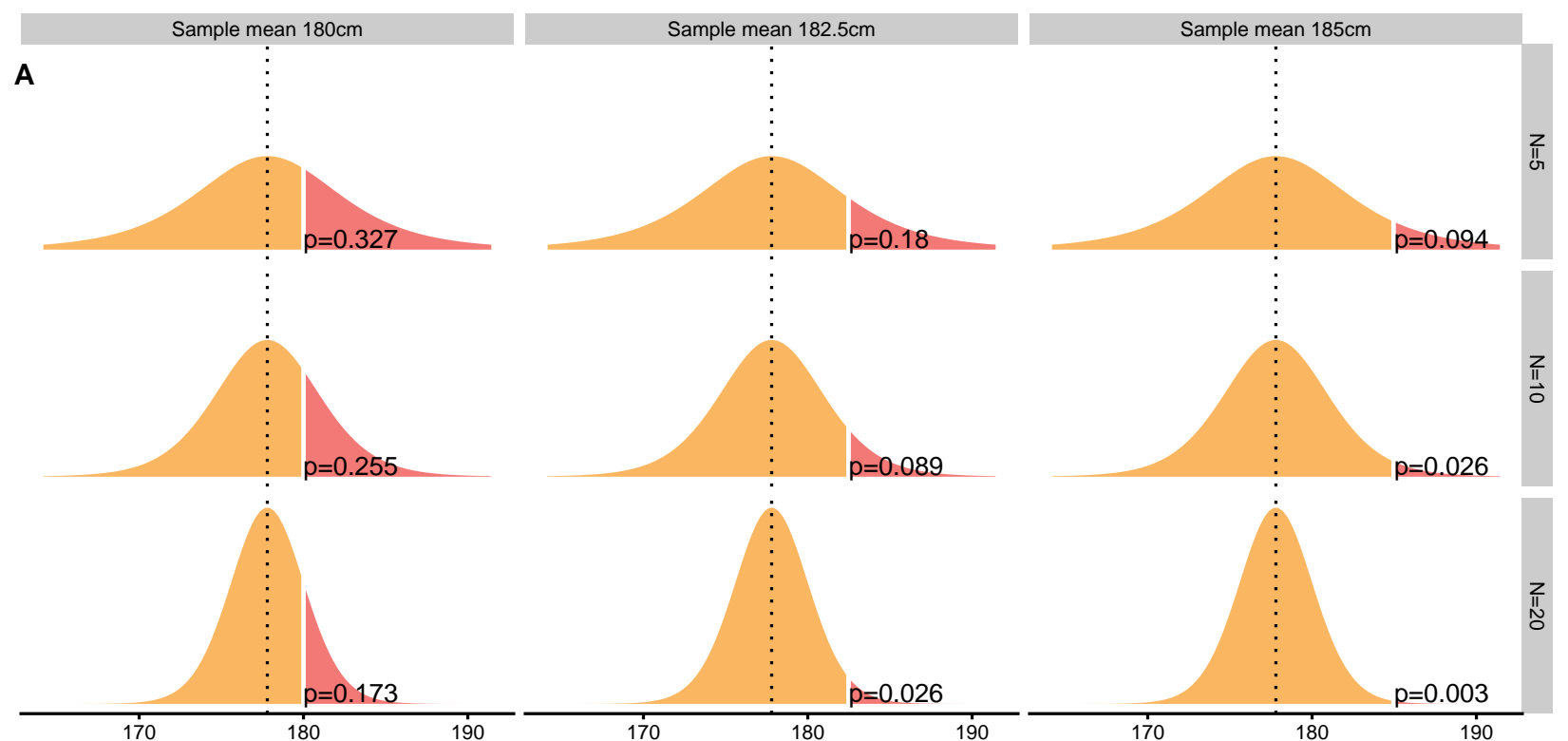

B

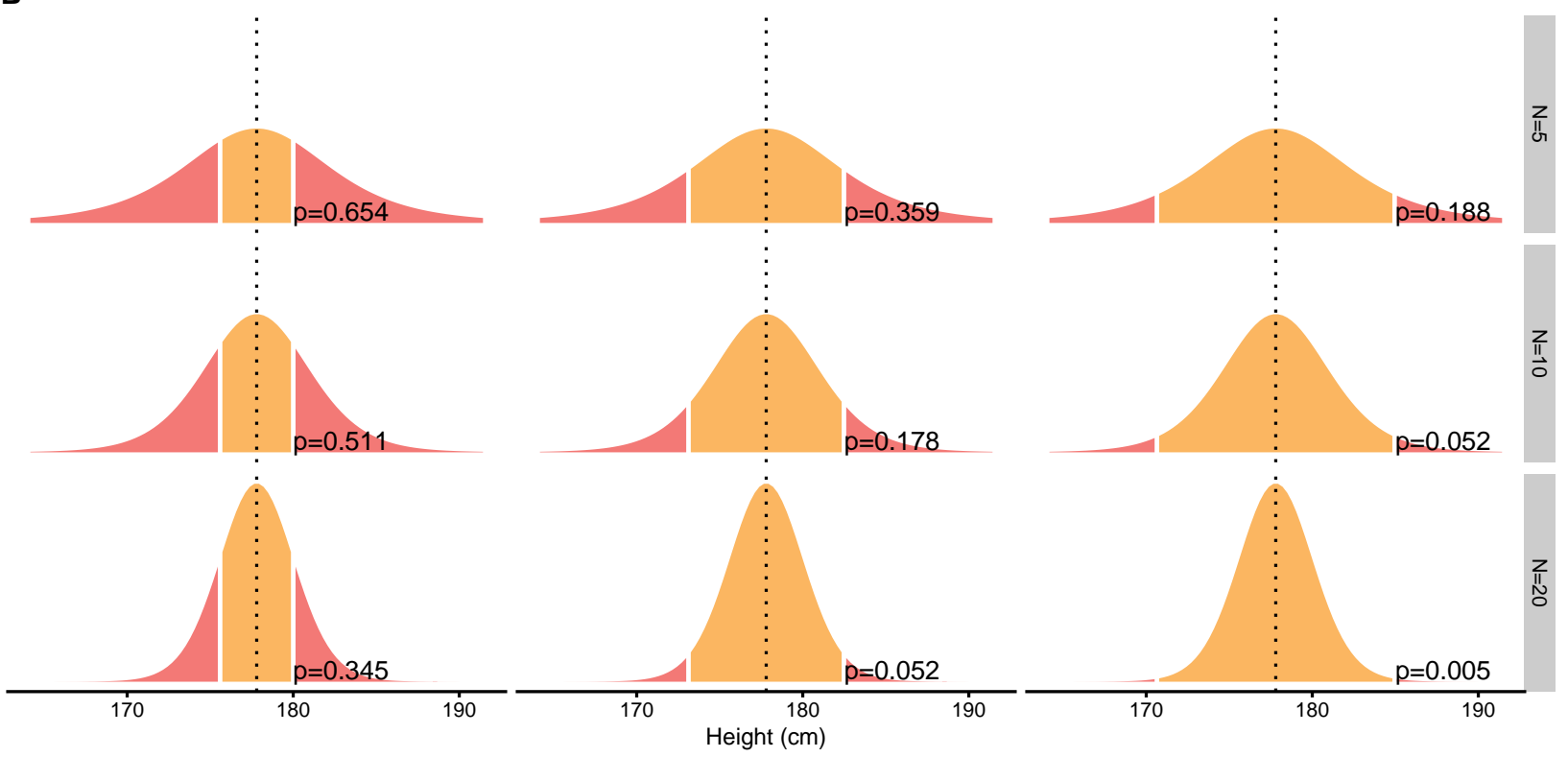

Figure 25. Null-hypothesis significance testing. Assuming null-hypothesis (true parameter value, or parameter value in the population, in this case mean or $\mu$ ) is true, probability of observing sample parameter of a given magnitude or larger, is estimated by calculating proportion of sampling distribution that is over sample parameter value. The larger the sample size, the smaller the width of the sampling distribution. A. One-sided approach is used when we are certain about the direction of the effect. B. Two-sided approach is used when we are unsure about the effect direction

The calculated probability of observing sample mean or larger, given null-hypothesis, is called p-value. In other words, p-value is the probability of observing data (in this case sample mean) given the null hypothesis (equation 36):

$$
p \text { value }=p(\text { Data } \mid H 0)
$$


It is easy to interpret p-values as "probability of the null hypothesis (given data)" ( $p(H 0 \mid D a t a)$ ), but that is erroneous. This is Bayesian interpretation (also called inverse probability) which is quite common, even for the experienced researchers. Unfortunately, p-values cannot be interpreted in such ways, but rather as a "probability of data given null hypothesis".

As you can see from the Figure 25, for the same difference in sample mean height, different sample sizes will produce different p-values. This is because sampling distribution of the mean will be narrower (i.e. smaller SEM) as the sample size increase. In other words, for the same effect (in this case sample mean), p-value will be smaller as the sample size gets bigger. It is thus important to realize that p-values don't tell us anything about the magnitude of the effect (in this case the difference between the sample mean and the known population mean).

The procedures of acquiring p-values are called statistical tests. With the example above, we are using one variation of the Student $t$ test, where we are calculating the test value $t$ using the following equation (37):

$$
\begin{aligned}
& t=\frac{\bar{x}-\mu}{S E M} \\
& t=\frac{\bar{x}-\mu}{\frac{S D}{\sqrt{N}}}
\end{aligned}
$$

P-value is then estimated by using the calculated t value and appropriate Student's t distribution (which opposed to Figure 25, is centered around 0 and with SD equal to SEM) to calculate the surface area over a particular value of $t$. This is usually done in the statistical program, or by using tables similar to Table 34 . Once the p-value is estimated, we need to decide whether to reject the null-hypothesis or not. Does our sample mean comes from the population? In order to do that, we need to define the error we are willing to accept. This error is called alpha (Greek $\alpha$ ) or Type $I$ error and refers to making an error of rejecting the null-hypothesis when null-hypothesis is true. Out of sheer convenience, alpha is set to 0.1 (10\% error), 0.05 (5\% error) or 0.01 ( $1 \%$ error).

If p-value is smaller than alpha, we will reject the null-hypothesis and state that the effect has statistical significance. The statistical significance has bad wording since it doesn't imply magnitude of the effect, only that the sample data come from a different population assumed by null-hypothesis.

Let's assume we have sample size of $\mathrm{N}=20$ where sample mean is equal to $185 \mathrm{~cm}$. Using the known population mean and SD, we get that $t=3.17$. Using two-sided test and alpha $=0.05$, can we reject the null-hypothesis? In order to do this we can refer to Table 34, and check that for $\mathrm{DF}=20$ (which is not exact, but it will serve the purpose), $95 \%$ of sampling distribution (which leaves $2.5 \%$ on each tail which is equal to $5 \%$ alpha) will be within \pm 2.08 . Since calculated $t=3.17$ is over \pm 2.08 , we can reject the null-hypothesis with alpha $=0.05$. Figure 25 (Panel B) depicts the exact $\mathrm{p}$-value for this example, which is equal to $\mathrm{p}=0.005$. Statistical software can calculate exact p-values, but before these we available, tables and procedure just describes were used instead.

\subsubsection{Statistical Power}

The is other type of error we can commit: Type II error or beta (Greek $\beta$ ). In order to understand Type II error, we need to assume alternate hypothesis or $\mathrm{Ha}$. Type II error refers to the error we make when we reject the alternate-hypothesis when alternate-hypothesis is true. Type I and Type II error are inversely related - the more we are willing to make Type I errors, the less likely we are going to make Type II errors, and vice versa (Table 35).

Table 35. Type I and Type II errors 


\begin{tabular}{lll}
\hline & True $H_{0}$ & True $H_{a}$ \\
\hline Rejected $H_{0}$ & Type I & \\
Rejected $H_{a}$ & & Type II \\
\hline
\end{tabular}

It is important to keep in mind that with NHST, we never accept any hypothesis, we either reject it or not. For example, we never say "null-hypothesis is accepted $(\mathrm{p}=0.23)$ ", but rather "null-hypothesis is not rejected $(\mathrm{p}=0.23) "$.

Assuming that alternate-hypothesis is true, probability of rejecting the null-hypothesis is equal to $1-\beta$. This is called statistical power and depends on the magnitude of the effect we are aiming to detect (or not-to-reject to correct myself). Figure 26 depicts multiple examples of one-sided and two-sided statistical power calculations given the known alpha of 0.05 and null-hypothesis for difference in sample mean height of $\pm 2.5, \pm 5$, and $\pm 7.5 \mathrm{~cm}(+2.5,+5$, and $+7.5 \mathrm{~cm}$ for one sided test $)$ for $\mathrm{N}=5, \mathrm{~N}=10$ and $\mathrm{N}=20$.

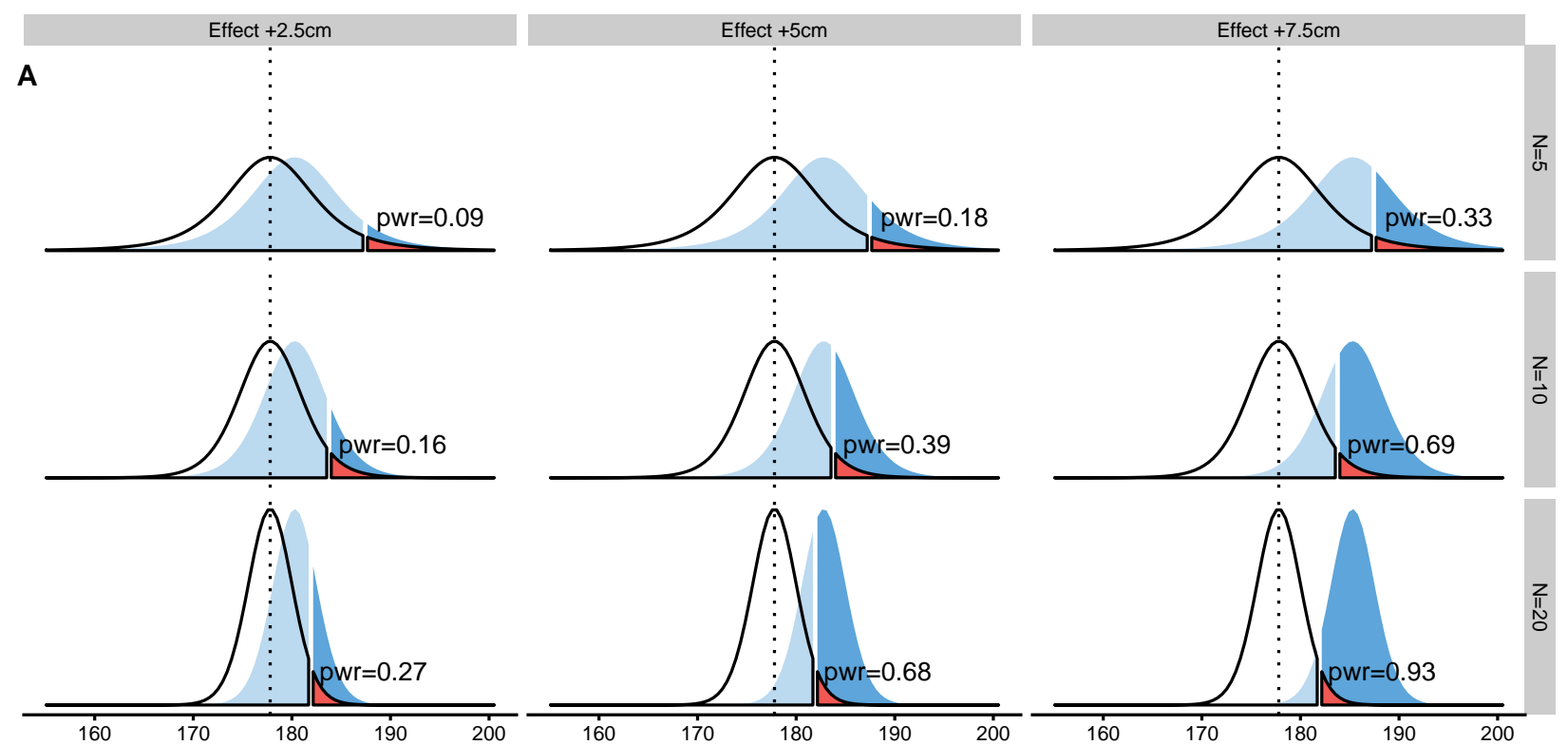

B
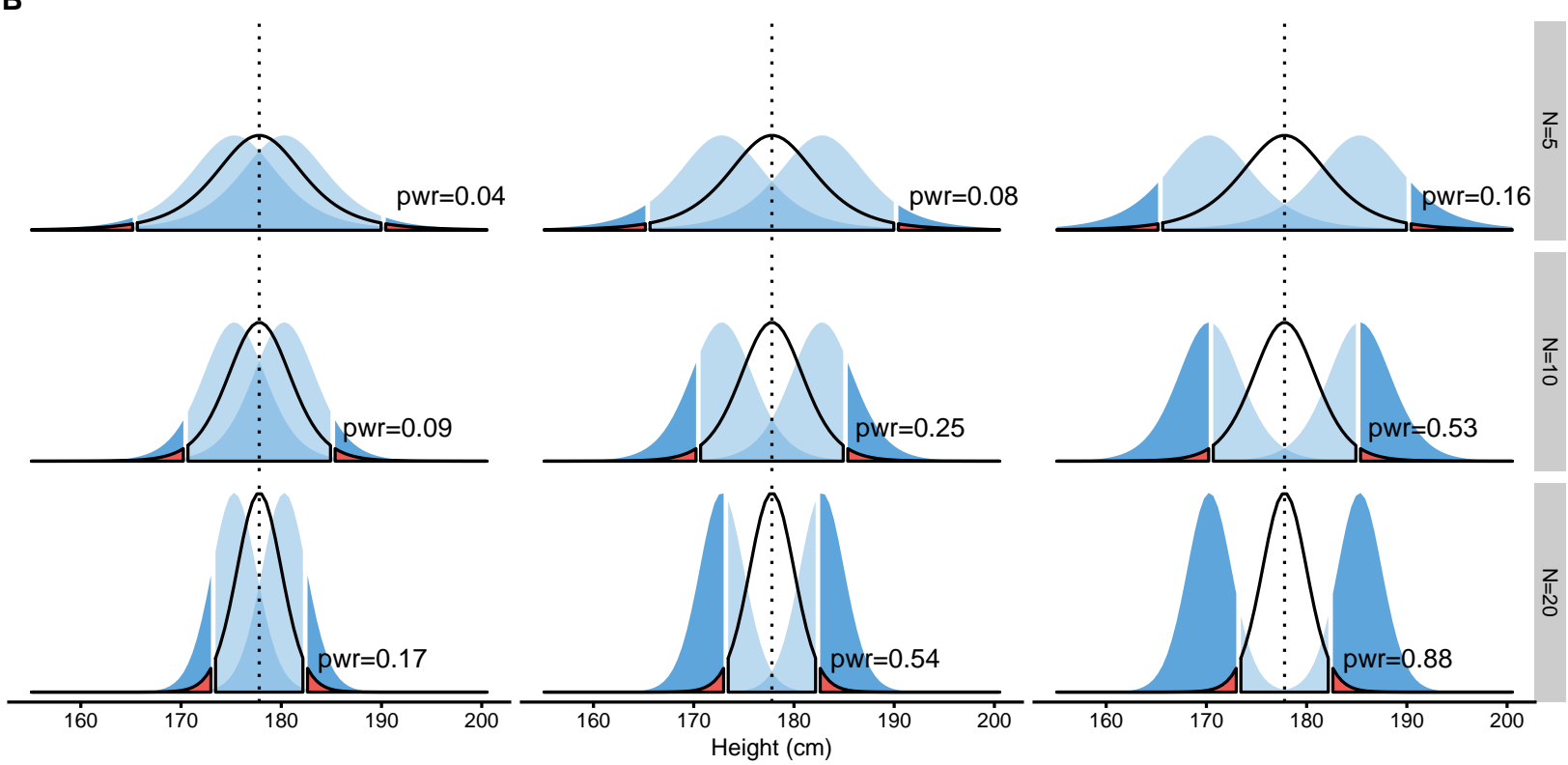
Figure 26. Statistical power. Statistical power is probability of detecting an effect of particular magnitude or larger. Visually, statistical power is dark blue surface and represents probability of rejecting the null-hypothesis given that the alternative hypothesis is true. A. One-sided approach. B. Two-sided approach

As can be seen from the Figure 26, the higher the magnitude of the effect (in this case difference in height means), the more likely we are to detect it (by rejecting null-hypothesis). Statistical power is mostly used when planning the studies to estimate sample size needed to detect effects of magnitude of interest (usually using known or observed effect from previous studies, or even SESOI). For example, question such as "How big of a sample do I need to detect $2.5 \mathrm{~cm}$ difference with $80 \%$ power, alpha 0.05 and known SD of $10 \mathrm{~cm}$ ?" is answered by using statistical power analysis. Statistical power, or sample size for needed statistical power can be easily calculated for simple analysis, but for some more elaborate simulations are needed.

The frequentist approach to statistical inference is all about maintaining accepted error rates, particularly Type I, for both tests and estimates. This can be particularly difficult when family-wise error rates need to be controlled, and these can emerge when multiple NHST are done. Some techniques, called p-hacking, can also introduce bias in the error rates by phishing for p-values (e.g. collecting samples until significant results are found). These topics are beyond the scope of this paper, but one of the reasons why some researchers prefer Bayesian inference.

\subsubsection{New Statistics: Confidence Intervals and Estimation}

Rather than performing NHST, uncertainty of the estimated parameter can be represented by the confidence interval (CI). CIs are usually pretty hard to explain and non-intuitive since they do not carry any distributional information. ${ }^{24}$ It is thus better to refer to CIs as compatibility intervals (Gelman \& Greenland, 2019), since, let's say $95 \%$ confidence interval contains all the hypotheses values of the parameter that would not be rejected by $\mathrm{p}<0.05$ NHST (Kruschke \& Liddell, 2018b). This implies that, in the long-run (when we repeat sampling infinite number of time), $95 \%$ confidence interval will capture true parameter value in $95 \%$ of the time.

Assuming $\mathrm{N}=20$ samples come from the population where the true mean height is equal to $177.8 \mathrm{~cm}$ and SD is equal to $10.16 \mathrm{~cm}$, if we calculate $95 \%$ CIs around sample parameter (in this case sample mean), in the long run, this CI will capture true population parameter in $95 \%$ of the time. Figure 27 depicts first 100 samples out of total of 1,000 taken from the population with calculated $95 \%$ CIs. CIs that missed the true population parameter value are depicted in red. Table 36 contain the summary for this simulation. If this simulation is repeated for many more times, CIs will capture true population parameter in $95 \%$, or in other words, have Type I error of $5 \%$.

${ }^{24}$ It is quite common to erroneously interpret CIs as Bayesian credible intervals (McElreath, 2015; Kruschke \& Liddell, 2018b). 


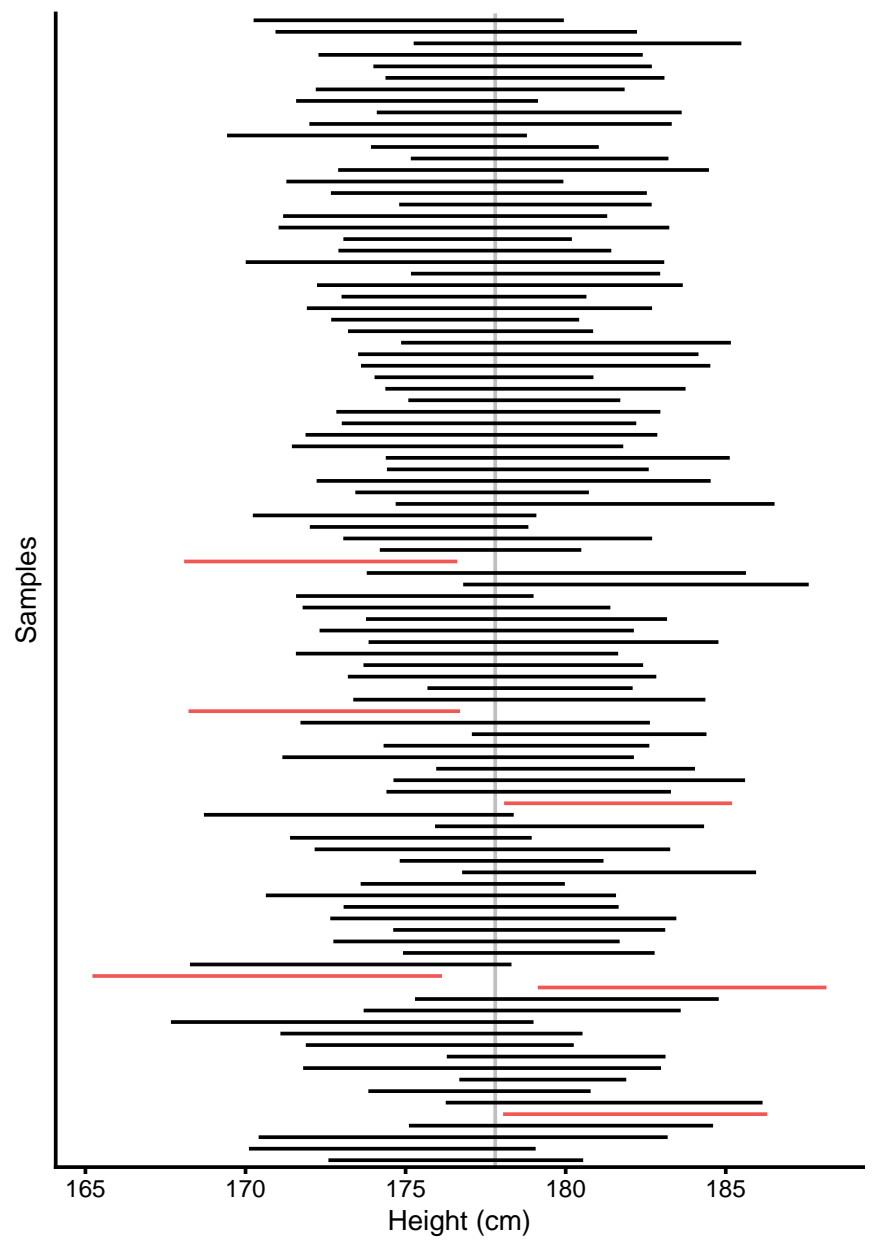

Figure 27. $95 \%$ confidence intervals for the sample mean, estimated for 100 samples $(\mathrm{N}=\mathbf{2 0}$ observations) drawn from population of known parameters (population mean is indicated by vertical line). In the long-run, $95 \%$ confidence intervals will span the true population value $95 \%$ of the time. Confidence intervals that didn't span the true population parameter value are colored in red

Table 36. Type I errors in 1000 samples

\begin{tabular}{rrr}
\hline Sample & Correct \% & Type I Errors \% \\
\hline 1000 & 96 & 4 \\
\hline
\end{tabular}

Similarly to different alphas, CIs can use different levels of confidence, usually 90\%, 95\%, 99\%. As already mentioned, mathematics behind the confidence intervals is equal to mathematics behind NHST. In order to calculate two-sided CIs for the sample mean, the following equation (38) is used:

$$
C I=\bar{x} \pm t_{\text {crit }} \times S \hat{E} M
$$

$T_{\text {crit }}$ can be found in the Table 34, where for $95 \%$ two-sided confidence $\mathrm{DF}=20$, is equal to 2.086. Using the example of observed sample mean of $185 \mathrm{~cm}$, known population SD and $\mathrm{N}=20$, calculated $95 \%$ confidence 
interval is equal to 180.26 to $189.74 \mathrm{~cm}$. From the compatibility interpretation standpoint, this CI means that the hypotheses with values ranging from 180.26 to $189.74 \mathrm{~cm}$, will not be rejected with alpha $=0.05$.

Confidence intervals are great solution for visualizing estimates. Figure 28 depicts already used example in Figure 25 to depict two-sided and one-sided p-values, but this time it depicts CIs around the sample means. Please note that in scenarios where $95 \%$ CIs cross the null-hypothesis, NHST will yield $\mathrm{p}>0.05$. This means that null-hypothesis is not rejected and results are not statistically significant. CIs can be thus used to visually inspect and conclude whether or not the null-hypothesis would be rejected or not in NHST is performed.
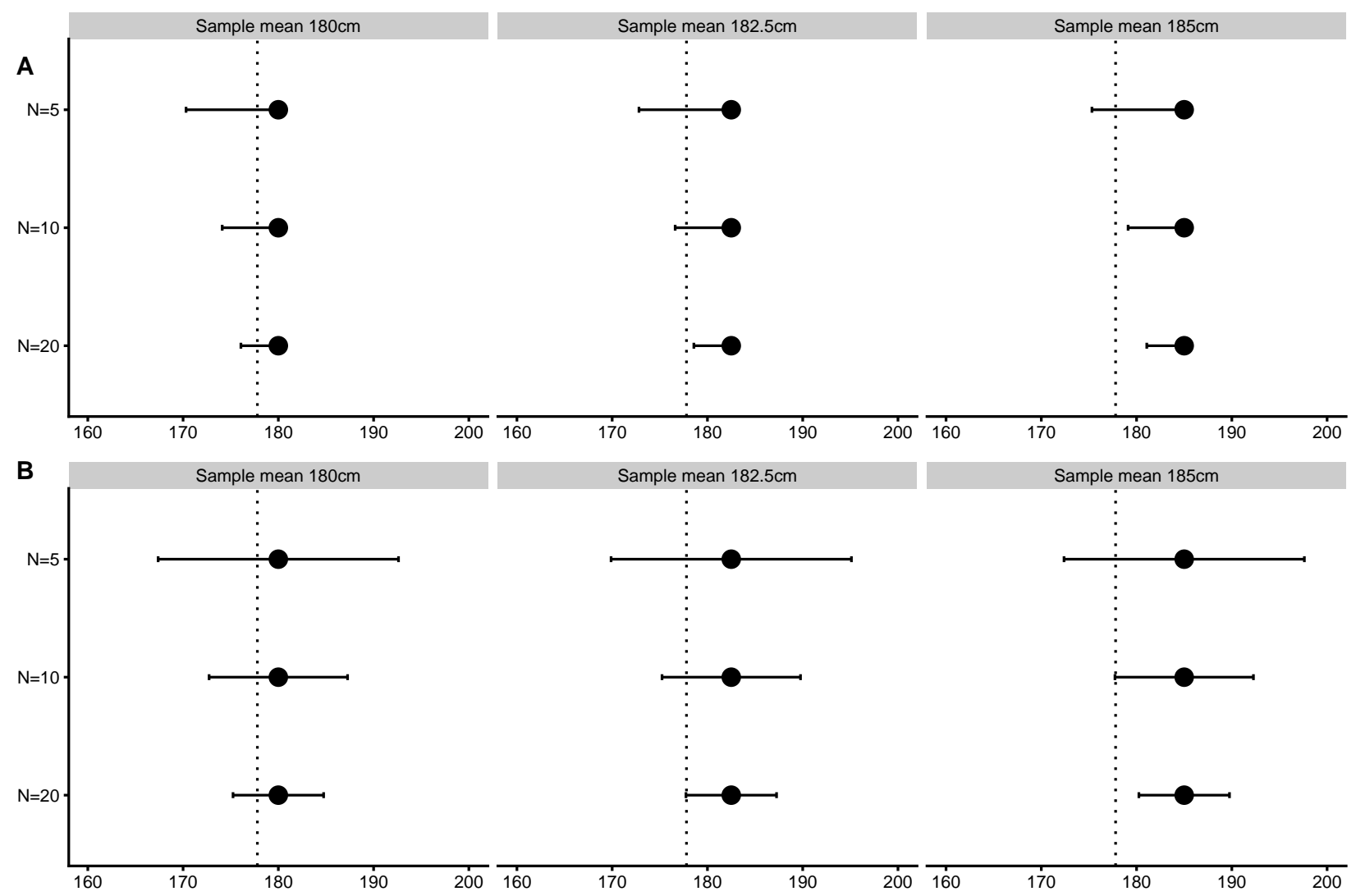

Figure 28. 95\% Confidence intervals for sample mean. Null-hypothesis of the population parameter value is indicated by vertical dashed line. If the $95 \%$ confidence interval doesn't touch or cross the null-hypothesis parameter value, p-value is less than 0.05 and effect is statistically significant (given alpha of 0.05). A. One-sided confidence intervals. B. Two-sided confidence intervals

\subsubsection{Minimum Effect Tests}

NHST doesn't tell us anything about the magnitudes of the effect. Just because the test is statistically significant $(\mathrm{p}<0.05)$, it's doesn't imply practically meaningful effect. Rather than using null-hypothesis of no effect, we can perform numerous one-sided NHST by using SESOI to infer practical significance. These are called minimum effect tests (METs) and can distinguish between 6 different conclusions: lower, not-higher, trivial, not-lower, higher and equivocal. Figure 29 depicts how SESOI and CIs can be used to distinguish between these 6 magnitude-based conclusions (Barker \& R. Schofield, 2008; Sainani et al., 2019). 


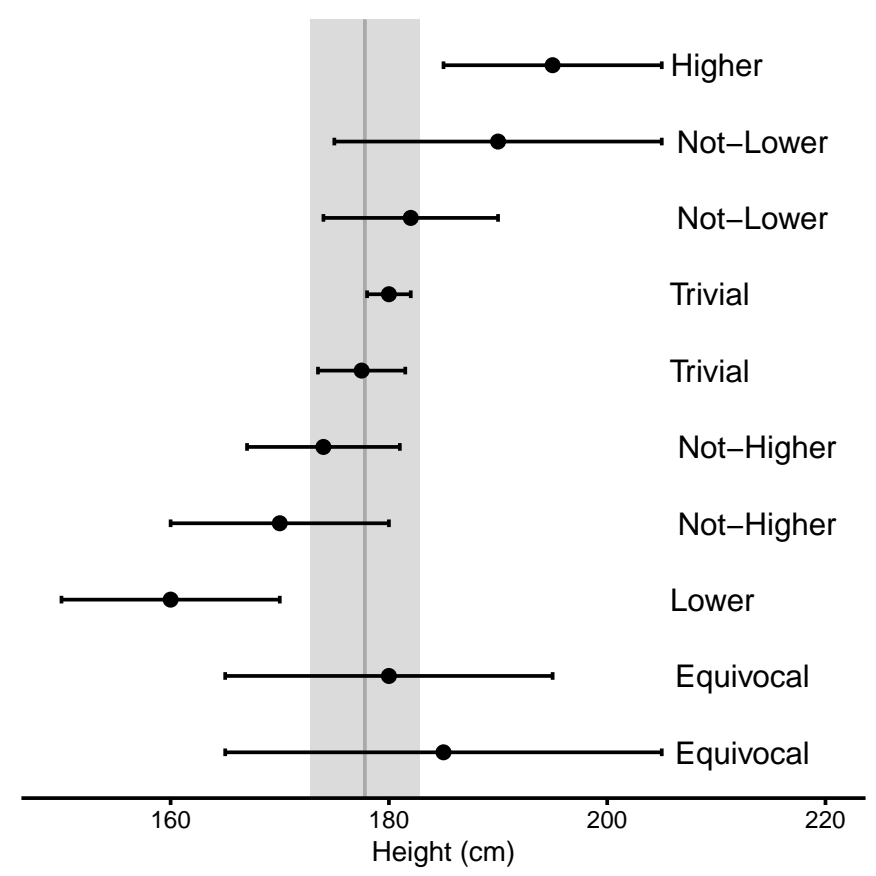

Figure 29. Inference about magnitudes of effects. Adapted and modified from Barker \& R. Schofield, 2008; Sainani et al., 2019

\subsubsection{Individual vs. Parameter SESOI}

So far we have used SESOI to infer practically significant differences or changes at the individual level. For example, answering what is the practically meaningful difference in height, which we used to calculate proportions and chances of observing indivuals with lower, trivial and higher magnitudes of effects.

In prediction tasks, SESOI was used to infer practically meaningful prediction error. This helped answering the question regarding whether the individual predictions are within practically equivalent region.

However, apart from using SESOI to infer individual change, difference, and prediction magnitudes, SESOI can also be used to evaluate statistics or parameters against practically significant anchor. For example, in equation 8, we have divided mean difference between the groups with SESOI to create magnitude-based estimator. But here, we assumed that the same magnitude used at the individual level is of equal practical importance at the group level. For example, individual change of $\pm 5 \mathrm{~kg}$ might be practically important at the level of the individual, but not at the level of the group, and vice versa. Usually, they are assumed to be the same (see also "Ergodicity" section).

Since sample mean difference is the estimator of the parameter in the population we are interested in estimating, we tend to use SESOI to give practical anchors for parameters as well. It could be argued that different terms should be used for the parameter SESOI (particularly for standardized estimators such as Cohen's d) versus individual SESOI. For example, we can use ROPE term for parameters (Kruschke \& Liddell, 2018a,b), and SESOI for individual-level magnitude inferences. For practical purposes they are considered equal, although I believe further discussion about this distintion is warranted.

\subsubsection{Two one-sided tests of equivalence}

Besides testing again null-hypothesis of no-effect, we can use the two one-sided tests (TOST) procedure to test for equivalence and reject the presence of the smallest effect size of interest (SESOI) (Lakens, 2017; Lakens, Scheel \& Isager, 2018). TOST involves using two one-sided NHST assuming parameter values as 
SESOI (Figure 30). Since the TOST produces 2 p-values, the larger of the two is reported. A conclusion of statistical equivalence is warranted when the larger of the two p-values is smaller than alpha Lakens (2017)], page 260 .

Utilizing estimation perspective to draw a conclusion of statistical equivalence, if the $90 \%$ (90\% not 95\%) CI falls completely within SESOI, conclusion of statistical equivalence at the level of alpha $=0.05$ can be warranted.
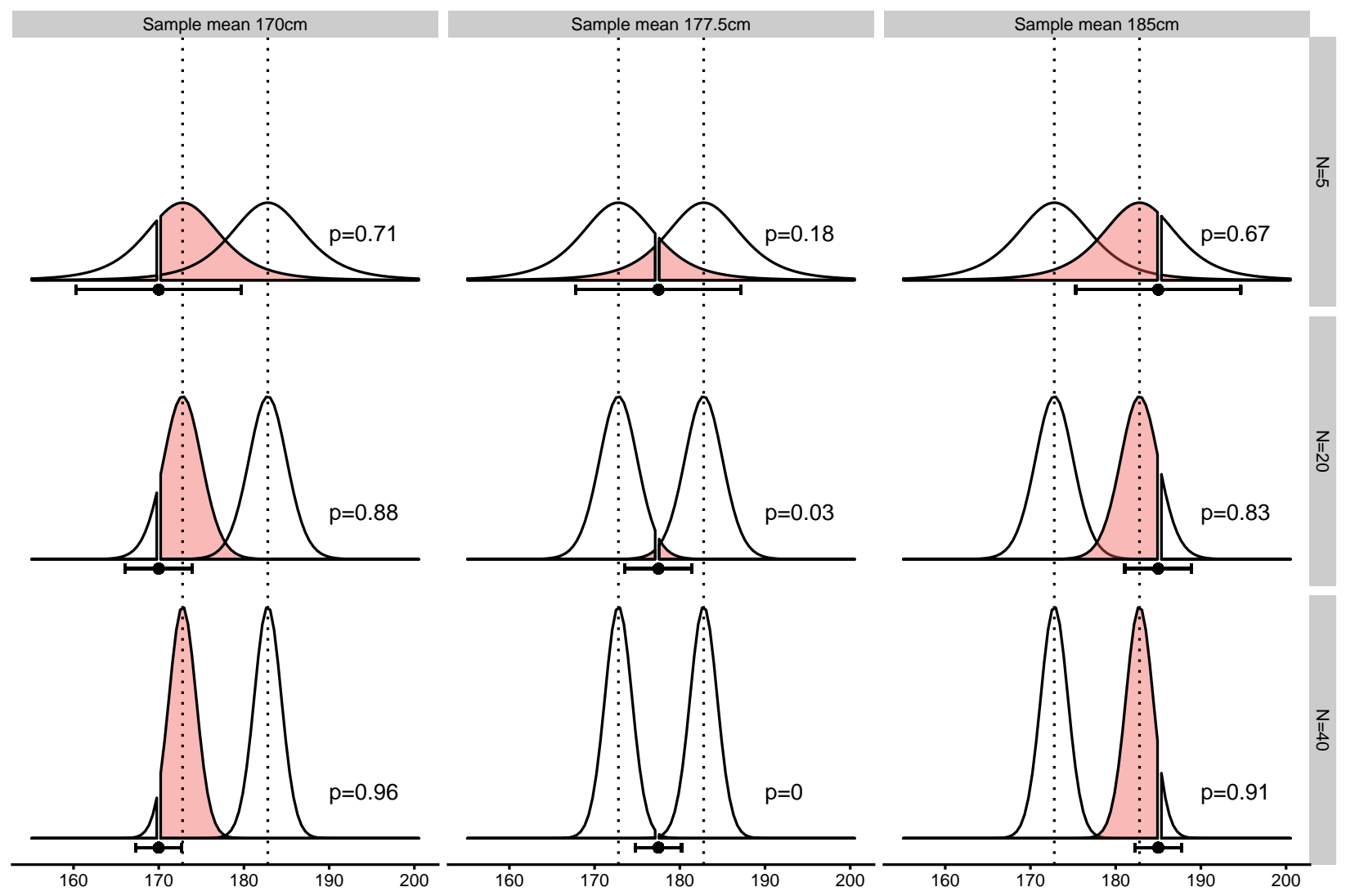

Figure 30. Equivalence test using two one-sided tests (TOST). Equivalence test assumes two null-hypotheses at SESOI thresholds and calculates two one-sided p-values, out of which a larger one is reported as result

\subsubsection{Superiority and Non-Inferiority}

Two same null-hypotheses at SESOI values are utilized to test superiority and non-inferiority of the effects. In other words, we want to conclude whether the effect is higher and/or not-lower than SESOI. To achieve this, two one-sided tests are performed to estimate the probability of observing effect in the positive direction (Figure 31). 


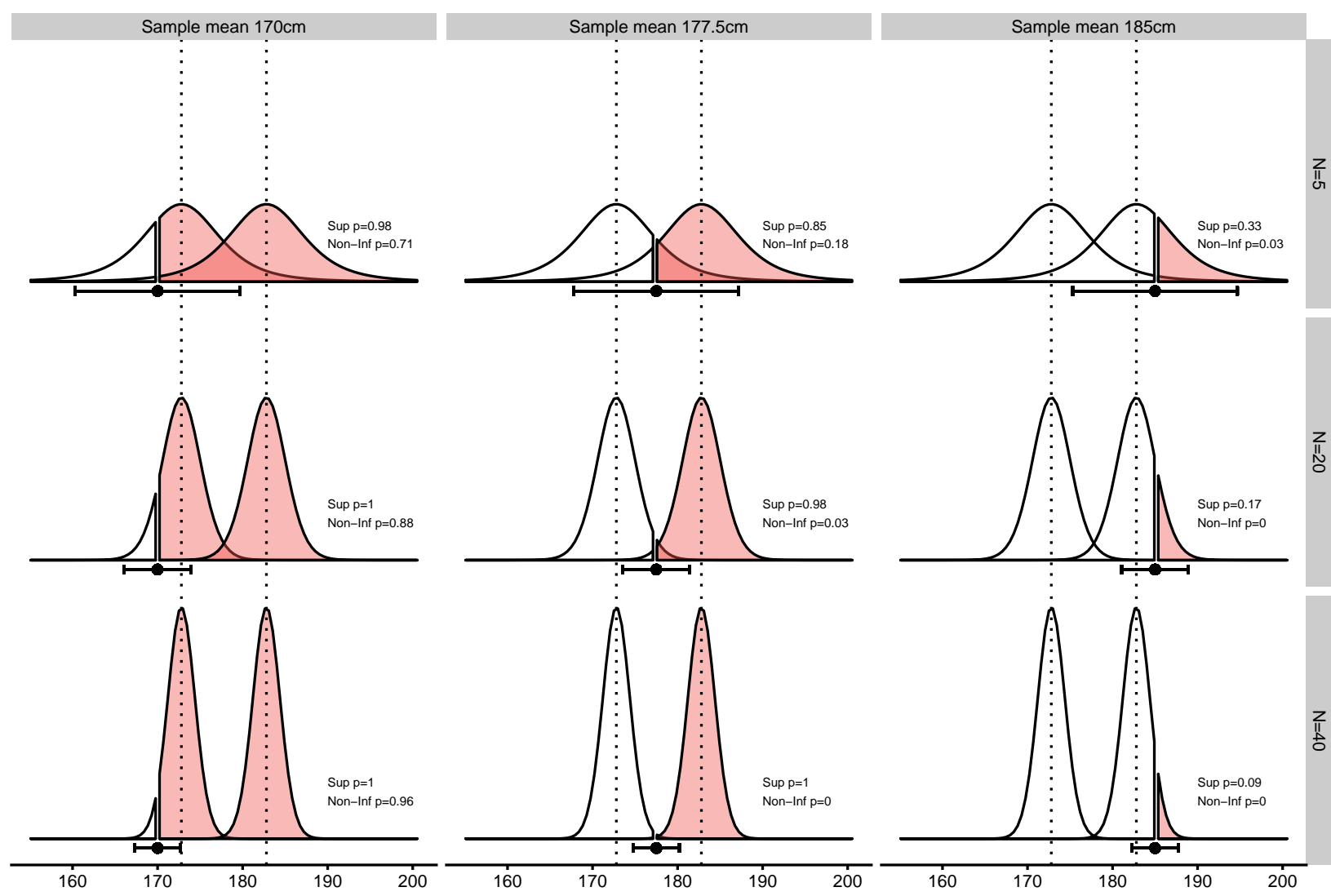

Figure 31. Superiority and Non-Inferiority tests. Similar to equivalence test using TOST procedure, superiority and non-inferiority tests involve one-sided NHST at SESOI thresholds in the positive direction

\subsubsection{Inferiority and Non-Superiority}

To test the inferiority and non-superiority of the effects, two one-sided tests are performed to estimate the probability of observing effect in the negative direction (Figure 32). 


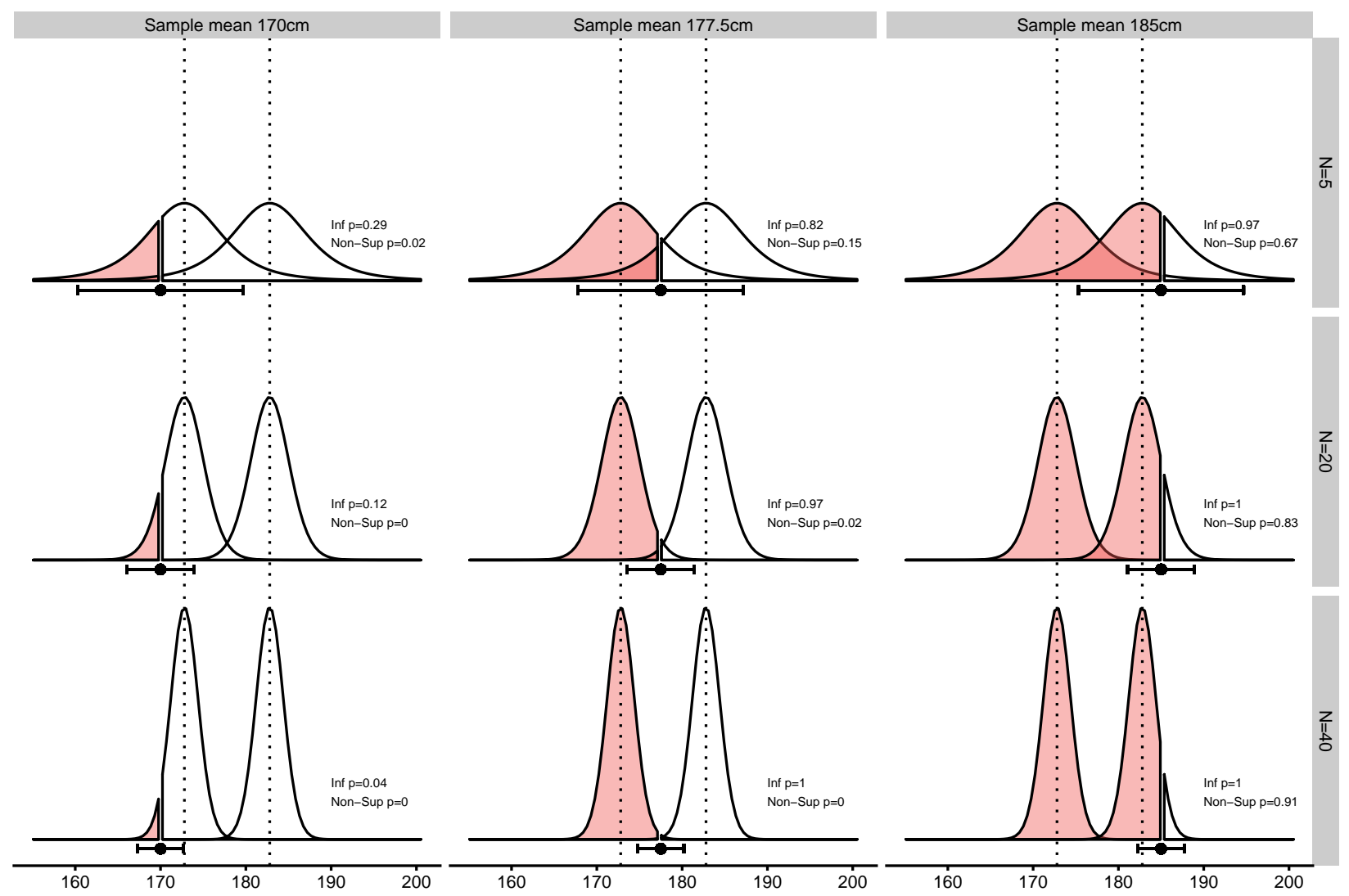

Figure 32. Inferiority and Non-Superiority tests. Similar to equivalence test using TOST procedure, inferiority and non-superiority tests involve one-sided NHST at SESOI thresholds in the negative direction

\subsubsection{Inference from METs}

The aforementioned tests provide five p-values: for lower (inferiority), not-higher (non-superiority), trivial (equivalent), not-lower (non-inferiority), and higher (superiority). These p-values can be used to device magnitude based inferences about the effects. Figure 33 depicts already used examples to calculate p-values from MET and the final inference on the magnitude of the effect. Values in the bracket indicate aforementioned p-values. 


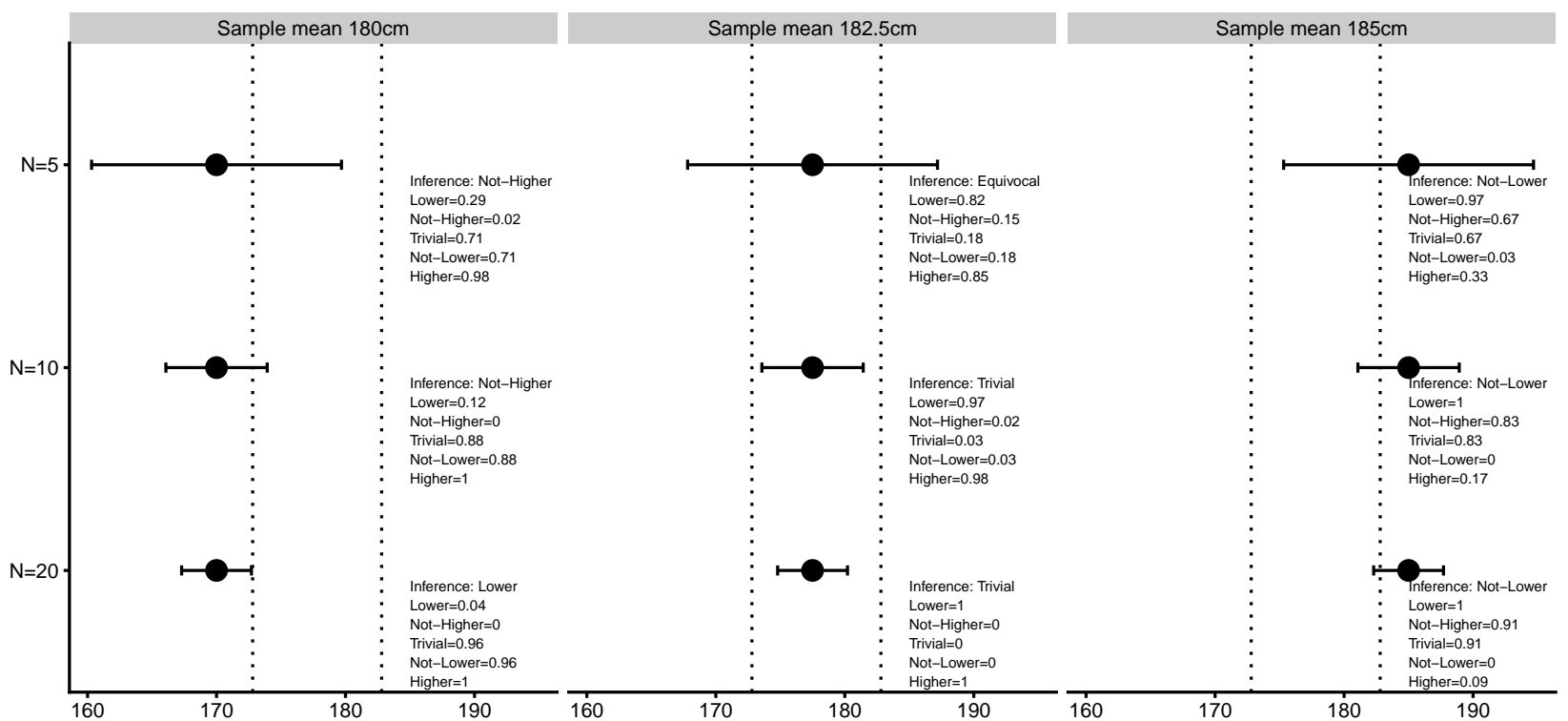

Figure 33. Minimum Effect Test results

\subsubsection{Magnitude Based Inference}

Batterham and Hopkins (Batterham \& Hopkins, 2006; Hopkins et al., 2009) proposed novel approach in making meaningful inference magnitudes, called magnitude based inference (MBI). MBI has been recently criticized (Barker \& R. Schofield, 2008; Welsh \& Knight, 2015; Hopkins \& Batterham, 2018; Curran-Everett, 2018; Sainani, 2018; Borg et al., 2018; Nevill et al., 2018; Sainani et al., 2019) for interpreting CIs as Bayesian credible intervals and for not controlling Type I and Type II errors.

As explained, CIs doesn't contain any probability distribution information about the true parameter. Although CIs, Bayesian credible intervals (with flat or non-informative prior), and bootstrap CI tend to converge to the approximately same values for very simple tests (such as t-test for the sample mean), interpreting CIs established using frequentist approach as Bayesian credible intervals is not valid approach to statistical inference (Sainani et al., 2019). Figure 34 depicts Bayesian interpretation of the confidence intervals used in MBI.

Using MBI as a simple descriptive approach to interpret CIs can be rationalized, but making inferences from estimated probabilities is not recommended (Caldwell \& Cheuvront, 2019). If frequentist approaches are used for statistical inference, METs should be used instead. 


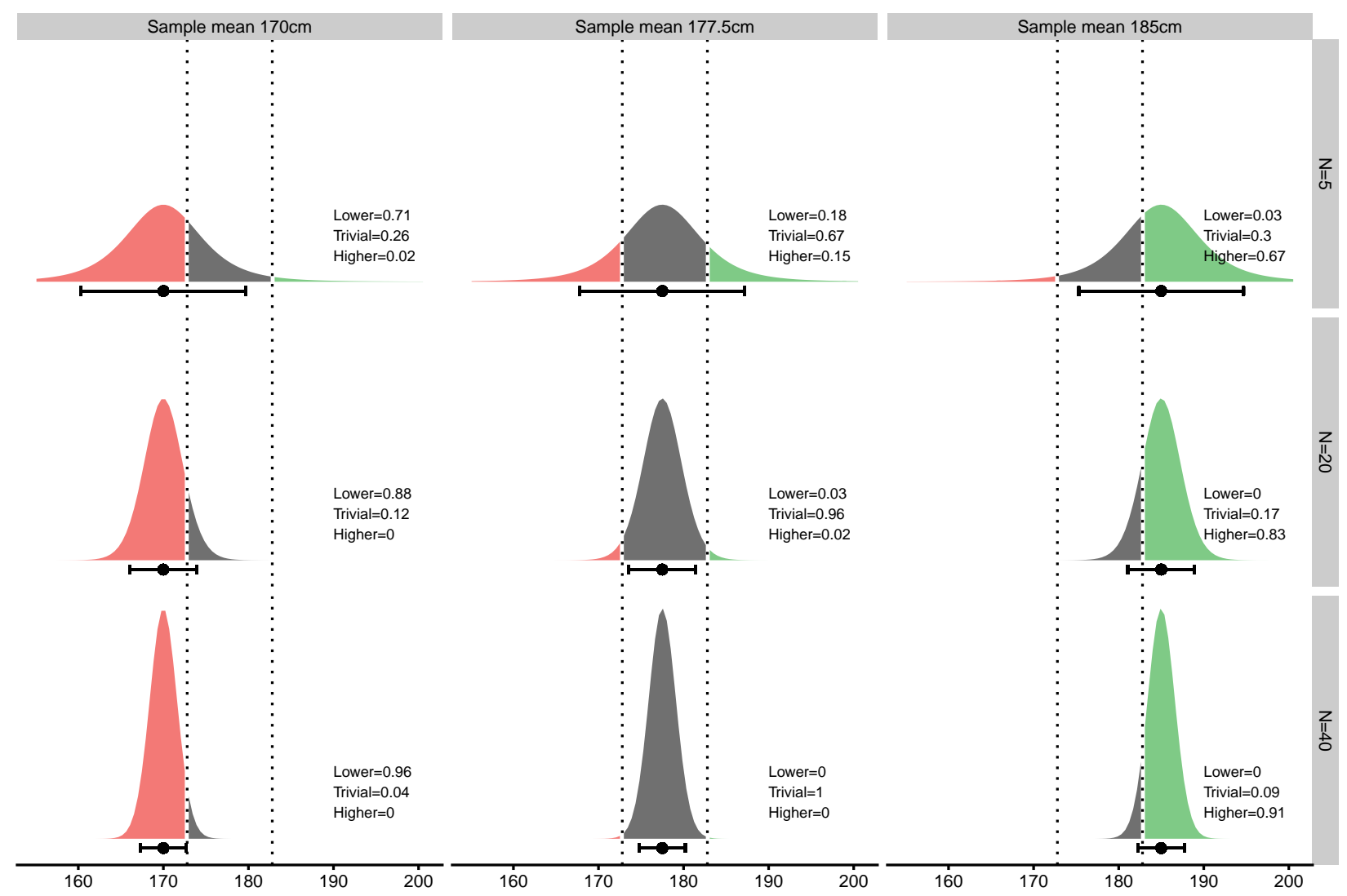

Figure 34. Magnitude-based inference use inappropriate Bayesian interpretation of the confidence intervals to calculate lower, trivial, and higher probabilities of the effect

There are numerous problems with frequentist inference (Kruschke \& Liddell, 2018b,a). The results are not intuitive and are usually erroneously interpreted from the Bayesian perspective. Error rates need to be controlled and adjusted when multiple comparisons are made, or when different stopping techniques are used, sampling distributions are unknown for some estimators and cannot be derived algebraically. Various assumptions such as assumptions of normality, non-colinearly and others, need to be made and tested for, and for more complex models, such as hierarchical models, p-values and CIs are only approximations. It is beyond this short review to delve into more details, regarding the frequentist approach to statistical inference, and readers are directed to references provided in this section.

\subsection{Bayesian perspective}

Bayesian inference is reallocation of plausibility (credibility) across possibilities (McElreath, 2015; Kruschke \& Liddell, 2018a,b). Kruschke and Liddell (Kruschke \& Liddell, 2018a) wrote in their paper as follows:

"The main idea of Bayesian analysis is simple and intuitive. There are some data to be explained, and we have a set of candidate explanations. Before knowing the new data, the candidate explanations have some prior credibility of being the best explanation. Then, when given the new data, we shift credibility toward the candidate explanations that better account for the data, and we shift credibility away from the candidate explanations that do not account well for the data. A mathematically compelling way to reallocate credibility is called Bayes' rule. The rest is just details." p. 156

The aim of this section is to provide the gross overview of the Bayesian inference using grid approximation method (McElreath, 2015), which is excellent teaching tool, but very limited for Bayesian analysis beyond 
simple mean and simple linear regression inference. More elaborate discussion on the Bayesian methods, such as Bayes factor, priors selection, model comparison, and Markov Chain Monte Carlo sampling is beyond the scope of this paper. Interested readers are directed to the references provided and suggested readings at the end of this paper.

\subsubsection{Grid approximation}

To showcase the rationale behind Bayesian inference let's consider the same example used in "Frequentist perspective" section - the male height. The question we are asking is, given our data, what is the true average male height ( $\mathrm{mu}$ or Greek letter $\mu$ ) and SD (sigma or Greek letter $\sigma$ ). You can immediately notice the difference in the question asked. In the frequentist approach we are asking "What is the probability of observing the data (estimate, like mean or Cohen's d), given the null hypothesis?"

True average male height and true SD represents parameters, and with Bayesian inference we want to relocate credibility across possibilities of those parameters (given the data collected). For the sake of this simplistic example, let's consider the following possibilities for the mean height: 170, 175, 180cm, and for SD: 9, 10, $11 \mathrm{~cm}$. This gives us the following grid (Table 37), which combines all possibilities in the parameters, hence the name grid approximation. Since we have three possibilities for each parameter, the grid consists of 9 total possibilities.

Table 37. Parameter possibilities

\begin{tabular}{rr}
\hline mu & sigma \\
\hline 170 & 9 \\
175 & 9 \\
180 & 9 \\
170 & 10 \\
175 & 10 \\
180 & 10 \\
170 & 11 \\
175 & 11 \\
180 & 11 \\
\hline
\end{tabular}

\subsubsection{Priors}

Before analyzing the collected data sample, with Bayesian inference we want to state the prior beliefs in parameter possibilities. For example, I might state that from previous data collected, I believe that the mean height is around 170 and $180 \mathrm{~cm}$, with a peak at $175 \mathrm{~cm}$ (e.g. approximating normal curve). We will come back to topic of prior later on, but for now let use uniform or vague prior, which assigns equal plausibility to all mean height and SD possibilities. Since each parameter has three possibilities, and since probabilities needs to sum up to 1 , each possibility has probability of $1 / 3$ or 0.333 . This is assigned to our grid in the Table 38 .

Table 38. Parameter possibilities with priors 


\begin{tabular}{rrrr}
\hline mu & sigma & mu prior & sigma \\
\hline 170 & 9 & 0.33 & 0.33 \\
175 & 9 & 0.33 & 0.33 \\
180 & 9 & 0.33 & 0.33 \\
170 & 10 & 0.33 & 0.33 \\
175 & 10 & 0.33 & 0.33 \\
180 & 10 & 0.33 & 0.33 \\
170 & 11 & 0.33 & 0.33 \\
175 & 11 & 0.33 & 0.33 \\
180 & 11 & 0.33 & 0.33 \\
\hline
\end{tabular}

\subsubsection{Likelihood function}

The sample height data we have collected for $\mathrm{N}=5$ individuals is $167,192,183,175,177 \mathrm{~cm}$. From this sample we are interested in making inference to the true parameter values (i.e. mean and SD, or $\mu$ and $\sigma$ ). Without going into the Bayes theorem for inverse probability, the next major step is the likelihood function. Likelihood function gives us a likelihood of observing data, given parameters. Since we have 9 parameter possibilities, we are interested in calculating for each possibility the likelihood of observing the data. This is represented with a following equation (39):

$$
L(x \mid \mu, \sigma)=\prod_{i=1}^{n} f\left(x_{i}, \mu, \sigma\right)
$$

The likelihood of observing the data is calculated by taking the product (indicated by $\prod_{i=1}^{n}$ sign in the equation 39 of likelihood of observing individual scores. The likelihood function is normal probability density function (PDF), whose parameters are $\mu$ and $\sigma$ (see Figure 35). This function has the following equation (41)

$$
f\left(x_{i}, \mu, \sigma\right)=\frac{e^{-(x-\mu)^{2} /\left(2 \sigma^{2}\right)}}{\sigma \sqrt{2 \pi}}
$$

Let's take a particular possibility of $\mu$ and $\sigma$, e.g. $175 \mathrm{~cm}$ and $9 \mathrm{~cm}$, and calculate likelihoods for each observed score (Table 39).

Table 39. Likelihoods of observing scores given $\mu$ and $\sigma$ equal to $175 \mathrm{~cm}$ and $9 \mathrm{~cm}$

\begin{tabular}{rrrr}
\hline mu & sigma & $\mathrm{x}$ & likelihood \\
\hline 175 & 9 & 167 & 0.02986 \\
175 & 9 & 192 & 0.00745 \\
175 & 9 & 183 & 0.02986 \\
175 & 9 & 175 & 0.04433 \\
175 & 9 & 177 & 0.04325 \\
\hline
\end{tabular}

Now, to estimate likelihood of the sample, we need to take the product of each individual score likelihoods. However, now we have a problem, since the resulting will be very, very small number $\left(1.272648 \times 10^{-8}\right)$. To solve this issue, we take the log of the likelihood function. This is called log likelihood (LL) and it is easier to compute without the fear of losing digits. Table 40 contains calculated log from the score likelihood.

Table 40. Likelihoods and log likelihoods of observing scores given $\mu$ and $\sigma$ equal to $175 \mathrm{~cm}$ and $9 \mathrm{~cm}$ 


\begin{tabular}{rrrrr}
\hline mu & sigma & $\mathrm{x}$ & likelihood & $\mathrm{LL}$ \\
\hline 175 & 9 & 167 & 0.02986 & -3.51122 \\
175 & 9 & 192 & 0.00745 & -4.90011 \\
175 & 9 & 183 & 0.02986 & -3.51122 \\
175 & 9 & 175 & 0.04433 & -3.11616 \\
175 & 9 & 177 & 0.04325 & -3.14085 \\
\hline
\end{tabular}

Rather than taking a product of the LL to calculate the overall likelihood of the sample, we take the sum. This is due the properties of the logarithmic algebra, where $\log x_{1} \times x_{2}=\log x_{1}+\log x_{2}$, which means that if we take the exponent of the sum of the log likelihoods, we will get the same result as taking the exponent of the product of likelihoods. Thus the overall log likelihood of observing the sample is equal to -18.18. If we take the exponent of this, we will get the same results as the product of individual likelihoods, which is equal to $1.272648 \times 10^{-8}$. This mathematical trick is needed to prevent very small numbers and thus loosing precision.

If we repeat the same procedure for every parameter possibility in our grid, we get the following log likelihoods (Table 41). This procedure is also visually represented in the Figure 35 for easier comprehension.

\section{Table 41. Sum of data log likelihoods for parameter possibilities}

\begin{tabular}{rrrrr}
\hline mu & sigma & mu prior & sigma prior & LL \\
\hline 170 & 9 & 0.33 & 0.33 & -20.12 \\
175 & 9 & 0.33 & 0.33 & -18.18 \\
180 & 9 & 0.33 & 0.33 & -17.78 \\
170 & 10 & 0.33 & 0.33 & -19.79 \\
175 & 10 & 0.33 & 0.33 & -18.21 \\
180 & 10 & 0.33 & 0.33 & -17.89 \\
170 & 11 & 0.33 & 0.33 & -19.63 \\
175 & 11 & 0.33 & 0.33 & -18.32 \\
180 & 11 & 0.33 & 0.33 & -18.06 \\
\hline
\end{tabular}




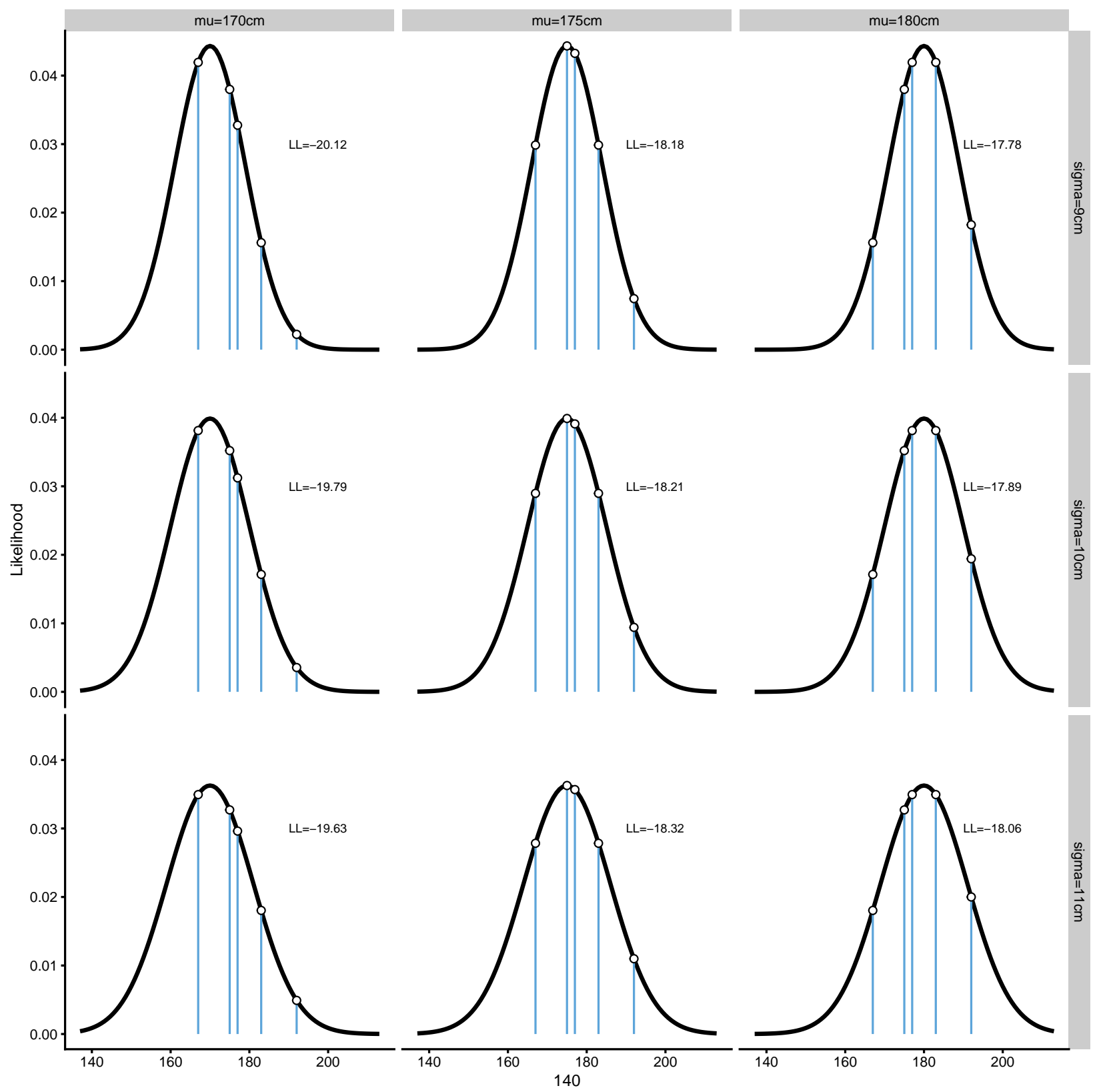

Figure 35. Likelihood of data given parameters. $\mu$ and $\sigma$ represent parameters for which we want to estimate likelihood of observing data collected

\subsubsection{Posterior probability}

To get the posterior probabilities of parameter possibilities, likelihoods need to be multiplied with priors $($ posterior $=$ prior $\times$ likelihood $)$. This is called Bayesian updating. Since we have log likelihoods, we need to sum the $\log$ likelihoods with $\log$ of priors instead $(\log$ posterior $=\log$ prior $+\log$ likelihood). To get the posterior probability, after converting log posterior to posterior using exponent $\left(\text { posterior }=e^{\log \text { posterior }}\right)^{25}$,

\footnotetext{
${ }^{25}$ There is one more mathematical trick done to avoid very small numbers explained in "Statistical Rethinking" (McElreath, 2015) and it involves doing the following calculation to get the posterior probabilities: posterior $=e^{\log \text { posterior-max(log posterior)) }}$.
} 
we need to make sure that probabilities of parameter possibility sum to one. This is done by simply dividing probabilities for each parameter possibility by the sum of probabilities.

Table 42 contains the results of Bayesian inference. The posterior probabilities are called joint probabilities since they represent probability of a combination of particular $\mu$ and $\sigma$ possibility.

Table 42. Estimated posterior probabilities for parameter possibilities given the data

\begin{tabular}{rrrrrr}
\hline mu & sigma & mu prior & sigma prior & LL & posterior \\
\hline 170 & 9 & 0.33 & 0.33 & -20.12 & 0.02 \\
175 & 9 & 0.33 & 0.33 & -18.18 & 0.14 \\
180 & 9 & 0.33 & 0.33 & -17.78 & 0.20 \\
170 & 10 & 0.33 & 0.33 & -19.79 & 0.03 \\
175 & 10 & 0.33 & 0.33 & -18.21 & 0.13 \\
180 & 10 & 0.33 & 0.33 & -17.89 & 0.18 \\
170 & 11 & 0.33 & 0.33 & -19.63 & 0.03 \\
175 & 11 & 0.33 & 0.33 & -18.32 & 0.12 \\
180 & 11 & 0.33 & 0.33 & -18.06 & 0.15 \\
\hline
\end{tabular}

Table 42 can be be converted into $3 \times 3$ matrix, with possibilities of $\mu$ in the columns, and possibilities of the $\sigma$ in the rows and posterior joint probabilities in the cells (Table 43). The sums of the joint probabilities in the Table 43 margins represent marginal probabilities for parameters.

Table 43. Joint distribution of the parameter possibilities. Sums at the table margins represent marginal probabilities

\begin{tabular}{lrrrr}
\hline & 170 & 175 & 180 & Sum \\
\hline 9 & 0.02 & 0.14 & 0.20 & 0.36 \\
10 & 0.03 & 0.13 & 0.18 & 0.34 \\
11 & 0.03 & 0.12 & 0.15 & 0.30 \\
Sum & 0.08 & 0.38 & 0.54 & 1.00 \\
\hline
\end{tabular}

Since we have only two parameters, the joint probabilities can be represented with the heat map. Figure 36 is a visual representation of the Table 43 . 


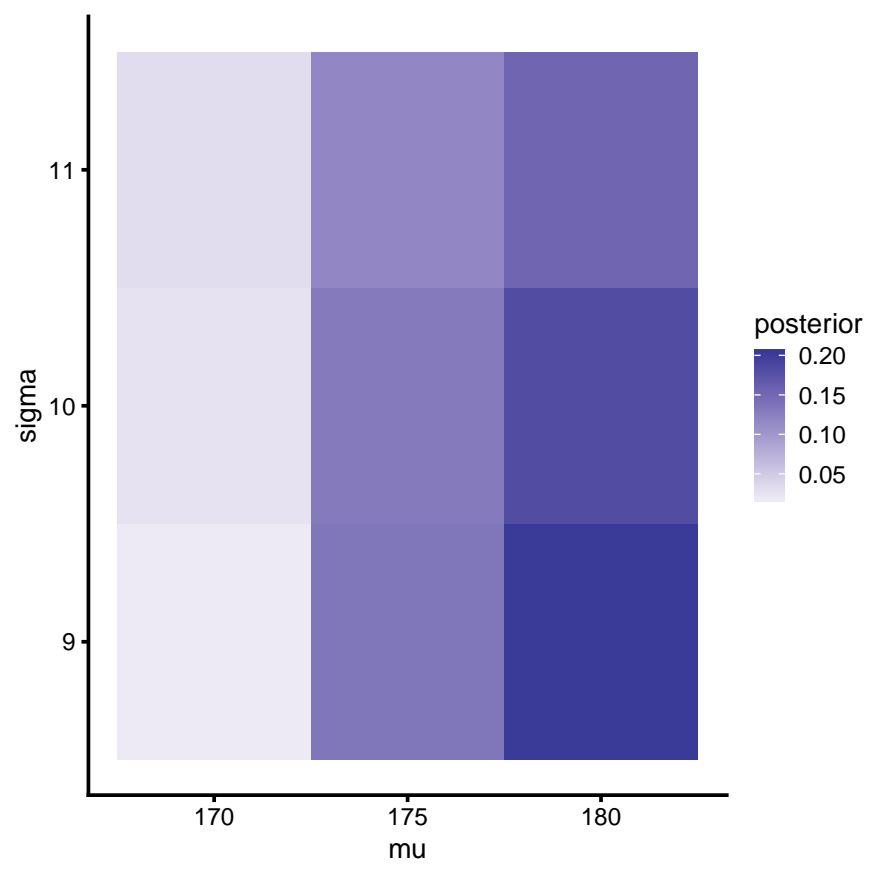

Figure 36. Heat map of $\mu$ and $\sigma$ joint probabilities

When we have more than 2 parameters, visualization of joint probabilities get's tricky and we rely on visualizing marginal posterior probabilities of each parameter instead. As explained, marginal probabilities are calculated by summing all joint probabilities for a particular parameter possibility. Figure 37 depicts marginal probabilities (including prior probabilities) for $\mu$ and $\sigma$. 


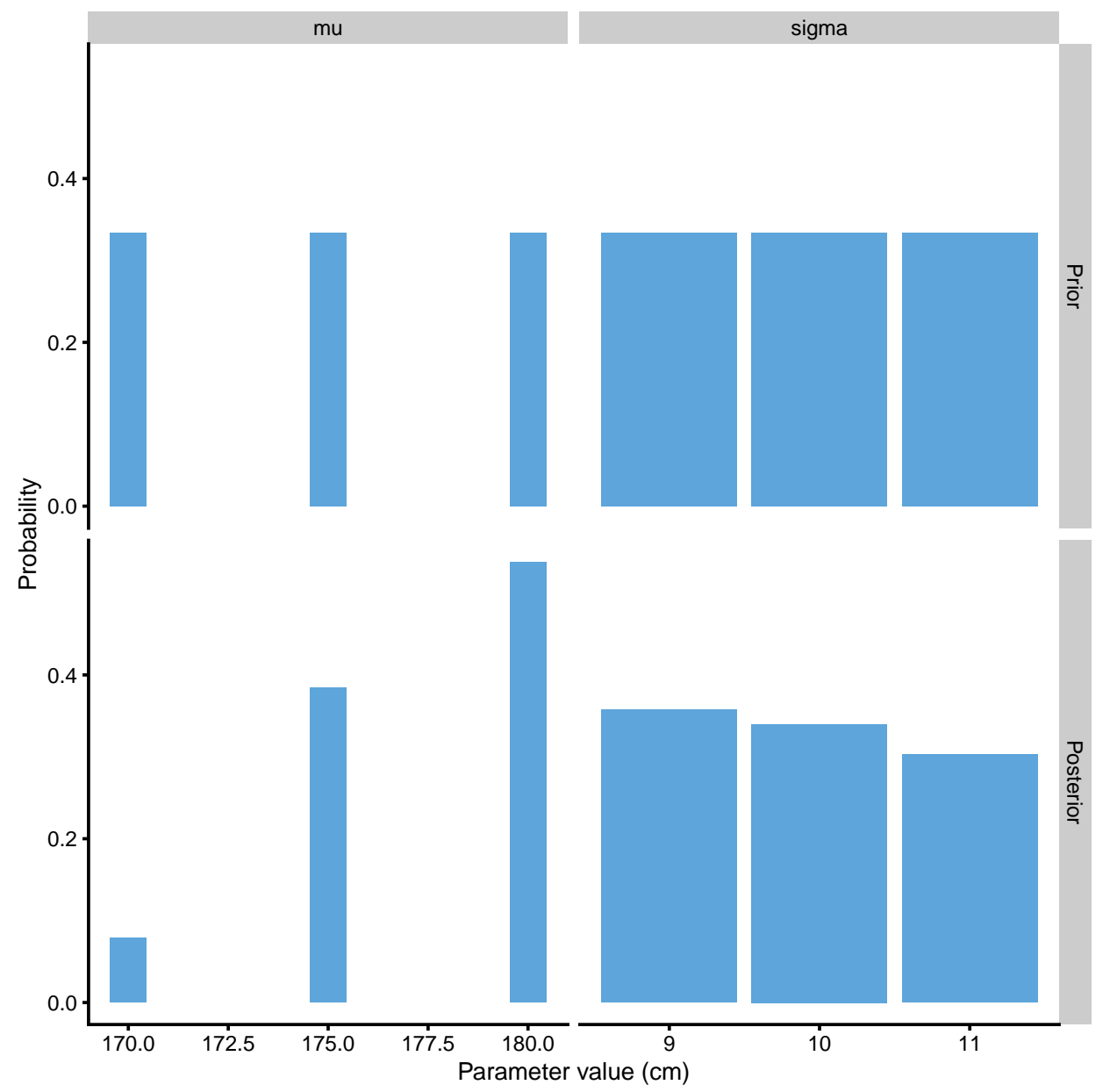

Figure 37. Prior and posterior distributions resulting from simplified grid-approximation example

As can be seen from the Figures 36 and 37, the most likely parameter possibilities, given the data, are $\mu$ of $180 \mathrm{~cm}$ and $\sigma$ of $9 \mathrm{~cm}$.

\subsubsection{Adding more possibilities}

So far, we have made this very granular in order to be understood. However, since we are dealing with continuous parameters, performing grid approximation for more than 9 total parameter possibilities seems warranted. The calculus is exactly the same, as well as the sample collected, but now we will use the larger range for both $\mu(160-200 \mathrm{~cm})$ and $\sigma(1-30 \mathrm{~cm})$, each with 100 possibilities. We are estimating credibility for total of $100 \times 100=10,000$ parameter possibilities. Figure 38 depicts heat map for the joint probabilities, and Figure 39 depicts prior and posterior marginal distributions for each parameter. 


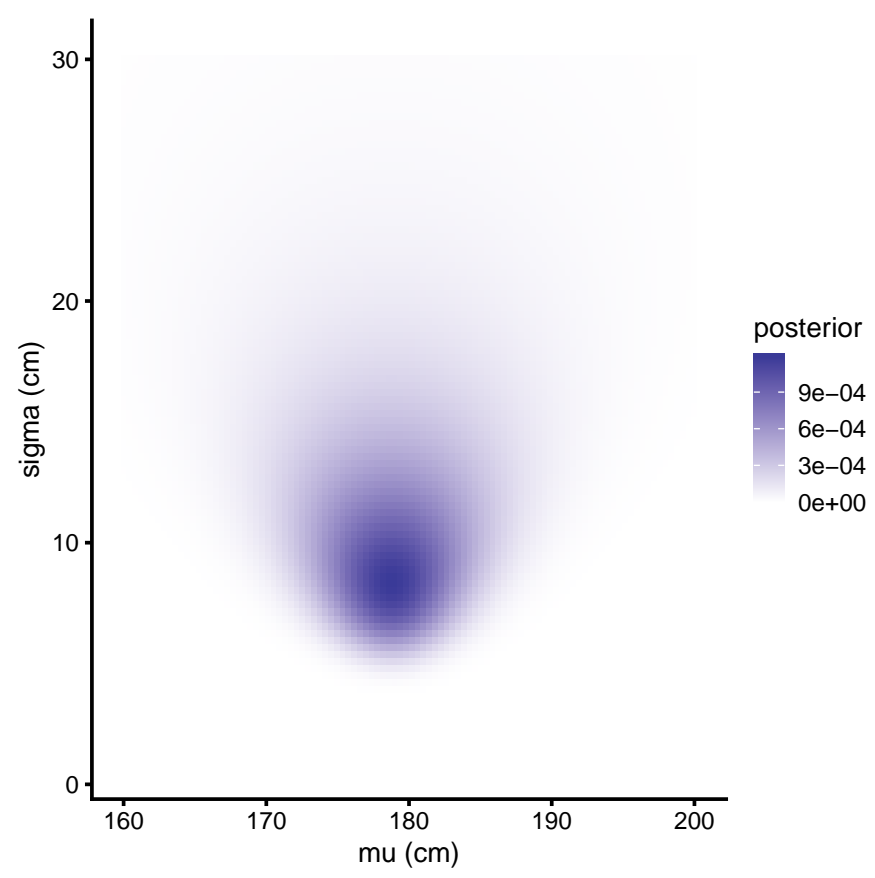

Figure 38. Heat map of $\mu$ and $\sigma$ joint probabilities when $100 \times 100$ grid-approximation is used

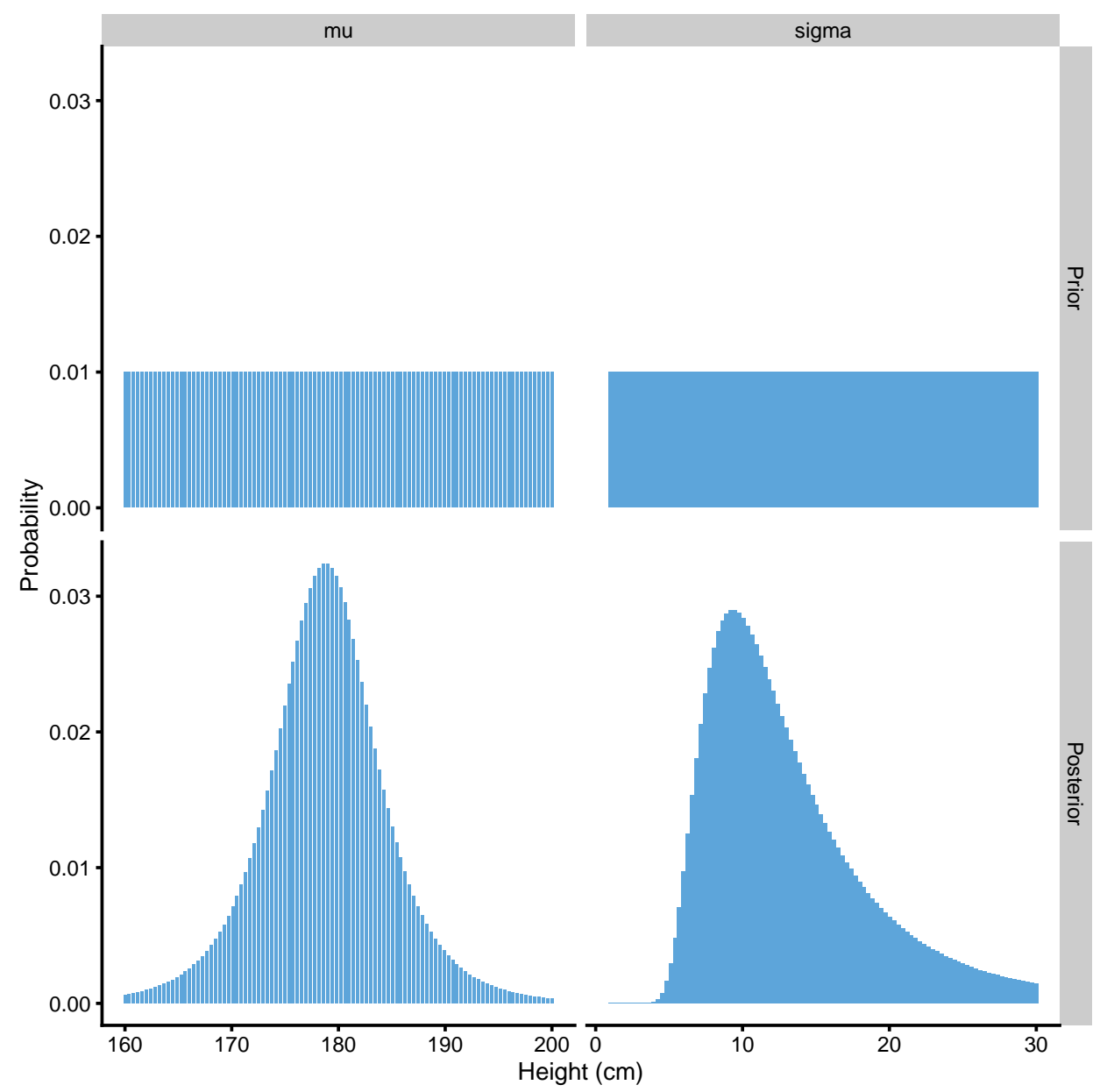




\section{Figure 39. Prior and posterior distributions resulting from $100 \times 100$ grid-approximation example}

Grid approximation utilized here is great for educational purposes and very simple models, but as number of parameters increases, the number of total parameter possibility grow so large, that it might take millions of years for a single computer to compute the posterior distributions. For example, if we have linear regression model with two predictors, we will have 4 parameters to estimate (intercept $\beta_{0}$, predictor one $\beta_{1}$, predictor two $\beta_{2}$, and residual standard error $\sigma$ ), and if we use 100 posibilities for each parameter, we will get $10^{\wedge} 8$ total number of possibilities.

This was the reason why Bayesian inference was not very practical. Until algorithms such as Markov Chain Monte Carlo (MCMC) emerged making Bayesian inference a walk in the park. Statistical Rethinking book by Richard McElreath is outstanding introduction into these topics.

\subsubsection{Different prior}

In this example we have used vague priors for both $\mu$ and $\sigma$. But let's see what happens when I strongly believe (before seeing the data), that $\mu$ is around $190 \mathrm{~cm}$ (using normal distribution with mean 190 and SD of 2 to represent this prior), but I do not have a clue about $\sigma$ prior distribution and I choose to continue using uniform prior for this parameter.

This prior belief if, of course, wrong, but maybe I am biased since I originate, let's say from Montenegro, country with one of the tallest men. Figure 40 contains plotted prior and posterior distributions. As can be seen, using very strong prior for $\mu$ shifted the posterior distribution to the higher heights. In other words, the data collected were not enough to overcome my prior belief about average height. 


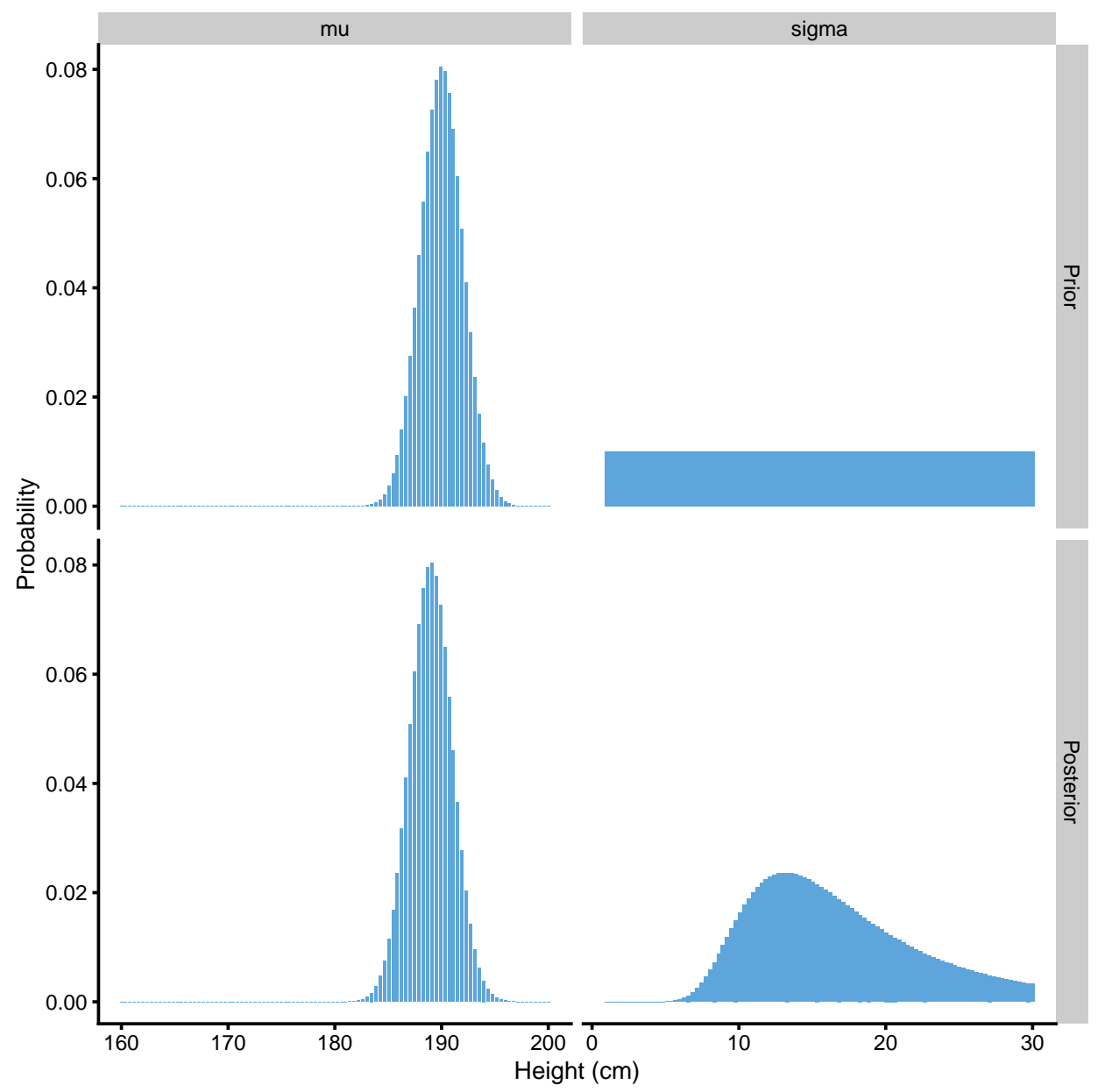

Figure 40. Effects of very strong prior on posterior

\subsubsection{More data}

The sample height data we have collected for $\mathrm{N}=5$ individuals $(167,192,183,175,177 \mathrm{~cm})$ was not strong to overcome prior belief. However, what if we sampled $\mathrm{N}=100$ males from known population of known mean height of 177.8 and SD of 10.16? Figure 41 depicts prior and posterior distributions in this example. As can be seen, besides having narrower posterior distributions for $\mu$ and $\sigma$, more data was able to overcome my strong prior bias towards mean height of $190 \mathrm{~cm}$. 


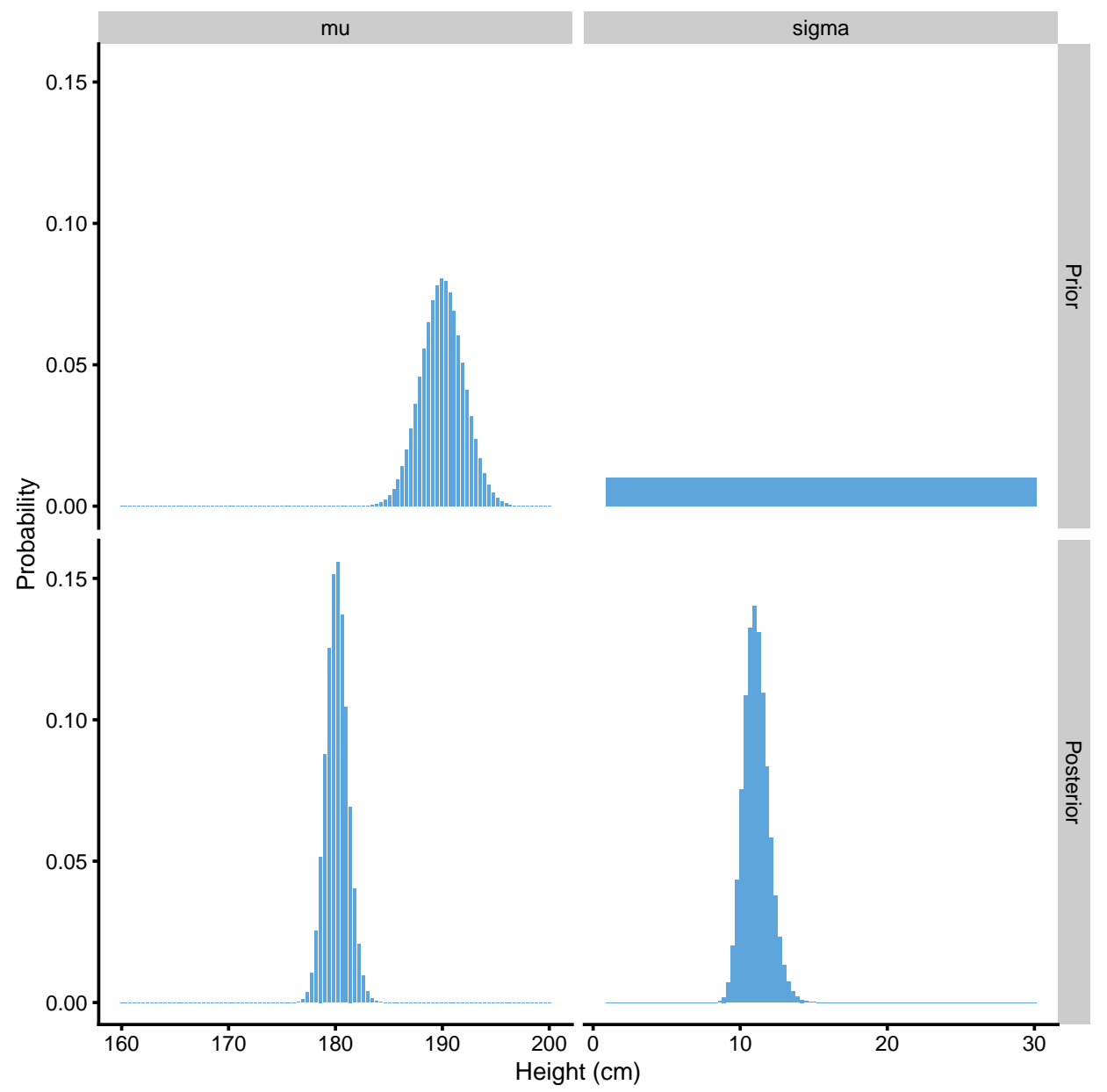

Figure 41. When larger sample is taken $(\mathrm{N}=100)$ as opposed to smaller sample $(\mathrm{N}=20)$, strong prior was not able to influence the posterior distribution

\subsubsection{Summarizing prior and posterior distributions with MAP and HDI}

In Bayesian statistics, the prior and posterior distributions are usually summarized using highest maximum a posteriori (MAP) and 90\% or 95\% highest density interval (HDI) (McElreath, 2015; Kruschke \& Liddell, 2018a,b). MAP is simply a mode, or the most probable point estimate. In other words, a point in the distribution with the highest probability. With normal distribution, MAP, mean and median are identical. The problems arise with distributions that are not symmetrical.

HDI is similar to frequentist CIs, but represents an interval which contains all points within the interval that have higher probability density than points outside the interval (Makowski, Ben-Shachar \& Lüdecke, 2019a,b). HDI is more computationally expensive to estimate, but compared to equal-tailed interval (ETI) or percentile interval, that typically excludes $2.5 \%$ or $5 \%$ from each tail of the distribution (for $95 \%$ or $90 \%$ confidence respectively), HDI is not equal-tailed and therefore always includes the mode(s) of posterior distributions (Makowski, Ben-Shachar \& Lüdecke, 2019a,b).

Figure 42 depicts comparison between MAP and 90\% HDI, median and 90\% percentile interval or ETI, and mean and ś1.64 $\times S D$ for $90 \%$ confidence interval. As can be seen from the Figure 42 , the distribution summaries differ since the distribution is asymmetrical and not-normal. Thus, in order to summarize prior or posterior distribution, MAP and HDI aremost often used, apart from visual representation. 

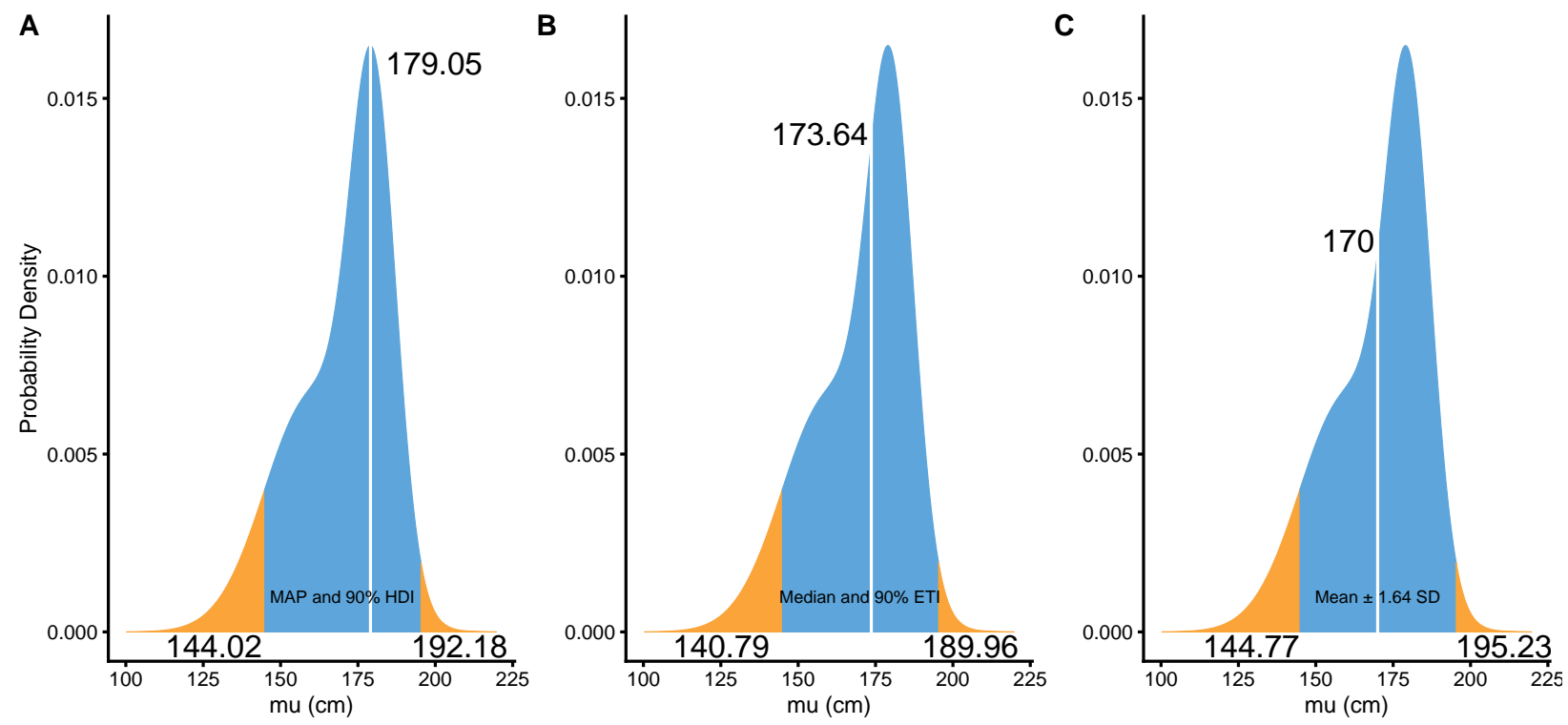

Figure 42. Summarizing prior and posterior distribution. A. MAP and 90\% HDI. B. Median and $90 \%$ ETI. C. Mean and $\pm 1.64 \times S D$

Using SESOI as a trivial range, or as a ROPE (Kruschke \& Liddell, 2018a,b), Bayesian equivalence test can be performed by quantifying proportion of posterior distribution inside the SESOI band (Kruschke \& Liddell, 2018a,b; Makowski, Ben-Shachar \& Lüdecke, 2019b; Makowski et al.). Magnitude based inference discussed in the section of the same name, would also be valid way of describing the posterior distribution.

Besides estimating using MAP and HDI, Bayesian analysis also allows to hypothesis testing using Bayes factor or MAP based p-value (Kruschke \& Liddell, 2018a,b; Makowski, Ben-Shachar \& Lüdecke, 2019b; Makowski et al.). Discussing these concepts is out of the range of this paper and interested readers can refer to references provided for more information.

\subsubsection{Comparison to NHST Type I errors}

How do the Bayesian HDIs compare to frequentist CIs? What are the Type I error rates when the data is sampled from the null-hypothesis? To explore this question, I will repeat the simulation from section 4.2.3 "New Statistics: Confidence Intervals and Estimation", where 1,000 samples of $\mathrm{N}=20$ observations are sampled from a population where the true mean height is equal to $177.8 \mathrm{~cm}$ and SD is equal to $10.16 \mathrm{~cm}$. Type I error is committed when the the $95 \%$ CIs or $95 \%$ HDI intervals of the sample mean don't cross the true value in the population. Table 44 contains Type I errors for frequentist and Bayesian estimation.

Table 44. Frequentist vs. Bayesian Type I errors

\begin{tabular}{lrrr}
\hline method & Sample & Correct \% & Type I Errors \% \\
\hline Frequentist & 1000 & 94.5 & 5.5 \\
Bayesian & 1000 & 95.1 & 4.9 \\
\hline
\end{tabular}

As can be seen from the Table 44, frequentist CI and Bayesian HDI Type I error rate are not identical (which could be due to the grid approximation method as well as due to only 1000 samples used). This is often a concern, since Bayesian methods do not control error rates (Kruschke \& Liddell, 2018a). Although 
frequentist methods revolve around limiting the probability of Type I errors, error rates are extremely difficult to pin down, particularly for complex models, and because they are based on sampling and testing intentions (Kruschke \& Liddell, 2018a). For more detailed discussion and comparison of Bayesian and frequentist methods regarding the error control see (Wagenmakers, 2007; Kruschke, 2013; Morey et al., 2016). Papers by Kristin Sainani (Sainani, 2018; Sainani et al., 2019) are also worth pondering about which will help in understanding estimation and comparison of Type I and Type II error rates between different inference methods, particularly when magnitude-based inference using SESOI is considered.

\subsection{Bootstrap}

As already stated, some estimators have unknown sampling distribution, particularly those that might have more practical use and answer predictive questions by the practitioners (e.g. "what is the proportion of athletes I can expect to demonstrate beneficial response due to this treatment?"). Hence, the frequentist approach is very hard to use. With the Bayesian approach, some estimators might be really hard to be modeled and represented, researchers might be confused with the priors and likelihood function selections, there is knowledge needed to understand and diagnose sampling chains from MCMC algorithms and so forth.

Bootstrap comes for the rescue (Davison \& Hinkley, 1997; Hesterberg, 2015; Efron \& Hastie, 2016; Canty \& Ripley, 2017; Rousselet, Pernet \& Wilcox, 2019b,a). Bootstrap is very simple and intuitive technique that is very easy to carry out. Let's take an example to demonstrate simplicity of the bootstrap. Continuing with the height example, let's assume that the following sample is collected for $\mathrm{N}=10$ individuals: 167, 175, 175, $176,177,181,188,190,197,211 \mathrm{~cm}$. We are interested in estimating the true mean in the population, SD in the population, and proportion of individuals taller than $185 \mathrm{~cm}$ (prop185; using algebraic method and estimated SD). The first step, as explained in the "Description" section of this paper, is to estimate those parameters using sample. But how do we estimate the uncertainty around the sample estimates?

Bootstrap involves resampling from the sample itself and then recalculating estimates of interest. If we have $\mathrm{N}=10$ observations in the collected sample, for each boostrap resample we are going to draw 10x1 observations. Some observations might be drawn multiple times, while some might not be drawn at all. This is then repeated numerous times, e.g., 2,000-10,000 times and for each bootstrap resample the estimators of interest are estimated. Table 45 contains 10 bootstrap resamples with calculated estimators of interest. Bootstrap resample of number 0 represents the original sample.

Table 45. Bootstrap resamples 


\begin{tabular}{rllrrr}
\hline Boot resample & Observations & mean & SD & prop185 \\
\hline 0 & 167175175176177181188190197211 & 183.7 & 13.00 & 0.46 \\
1 & 167167175177188190190190197211 & 185.2 & 13.81 & 0.51 \\
2 & 175176177177177188197197211211 & 188.6 & 14.50 & 0.60 \\
3 & 167175177177177177177181188197 & 179.3 & 8.08 & 0.24 \\
4 & 175175175175177177181188190197 & 181.0 & 7.90 & 0.31 \\
5 & 167167175175177177181188190197 & 179.4 & 9.75 & 0.28 \\
6 & 167167175175176188197197211211 & 186.4 & 16.86 & 0.53 \\
7 & 175175188188190190197197197211 & 190.8 & 10.75 & 0.71 \\
8 & 176176177188190190197197211211 & 191.3 & 13.03 & 0.69 \\
9 & 167167175176177188190197197211 & 184.5 & 14.49 & 0.49 \\
10 & 167167167175175181181181188190 & 177.2 & 8.47 & 0.18 \\
11 & 167167176176177177177197197211 & 182.2 & 14.48 & 0.42 \\
12 & 167175175177177181181190190197 & 181.0 & 8.93 & 0.33 \\
13 & 175177181188190190190190190211 & 188.2 & 9.93 & 0.63 \\
14 & 167175176176181188190190197211 & 185.1 & 12.83 & 0.50 \\
15 & 167167175175176177181181188197 & 178.4 & 9.08 & 0.23 \\
16 & 167175175176177177190190211211 & 184.9 & 15.40 & 0.50 \\
17 & 167175175175175175176177181188 & 176.4 & 5.32 & 0.05 \\
18 & 167175175181188188188190190197 & 183.9 & 9.12 & 0.45 \\
19 & 167175175175176177188190190211 & 182.4 & 12.63 & 0.42 \\
20 & 175175175177177188190190197211 & 185.5 & 12.04 & 0.52 \\
\hline
\end{tabular}

If we repeat this procedure 10,000 times, we can visualize bootstrap distribution of the estimators (Figure $43)$.
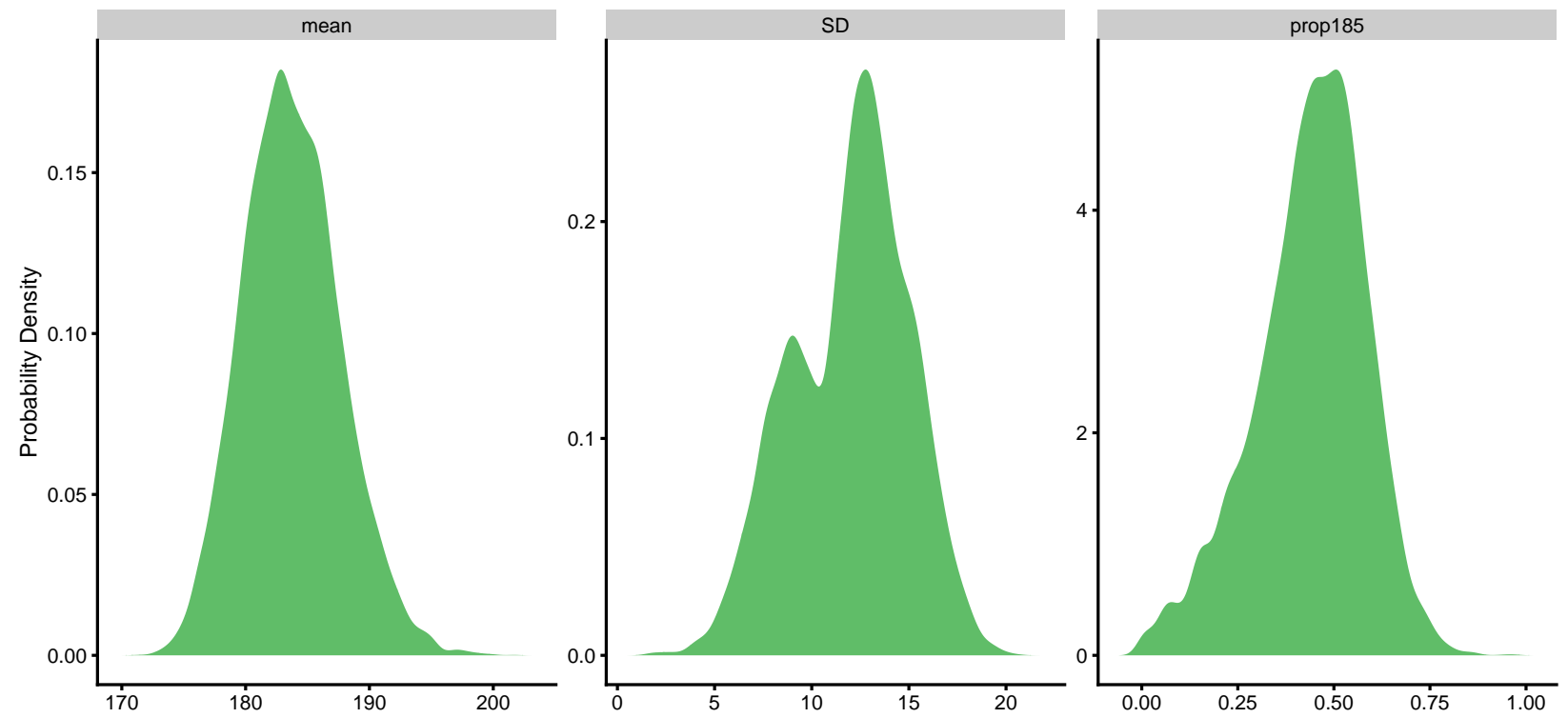

Figure 43. Bootstrap distribution of the estimators using 10,000 resamples

How should this bootstrap distribution be interpreted? In "Elements of Statistical Learning" (Hastie, Tibshirani \& Friedman, 2009), the following quote regarding bootstrap distribution can be found: 
"In this sense, the bootstrap distribution represents an (approximate) nonparametric, noninformative posterior distribution for our parameter. But this bootstrap distribution is obtained painlessly — without having to formally specify a prior and without having to sample from the posterior distribution. Hence we might think of the bootstrap distribution as a "poor man's" Bayes posterior. By perturbing the data, the bootstrap approximates the Bayesian effect of perturbing the parameters, and is typically much simpler to carry out" p. 272

Although the bootstrap was originally developed as a purely frequentist device (Efron, 2005), as per the quote above, it can be treated as "poor man's" Bayes posterior.

\subsubsection{Summarizing bootstrap distribution}

Bootstrap allows for both estimation and hypothesis testing. When it comes to estimations, point estimate of the bootstrap distribution is the sample parameter estimate. Confidence intervals around sample estimate are usually calculated using percentile approach (or ETI), or other approaches such as adjusted bootstrap percentile (BCa) (Davison \& Hinkley, 1997; Hesterberg, 2015; Efron \& Hastie, 2016; Canty \& Ripley, 2017; Rousselet, Pernet \& Wilcox, 2019b,a), or even HDI as used with Bayesian posterior distributions. In this paper I will utilize BCa intervals unless otherwise stated.

Hypothesis testing using the bootstrap distribution is possible through calculated p-value (Rousselet, Pernet \& Wilcox, 2019b,a). This not only allows for bootstrap NHST, but also all other MET, as well as MBI estimates (which assumes Bayesian interpretation of the bootstrap distributions). This is simply done by counting bootstrap sample estimates that are below or above particular threshold (i.e. null-hypothesis or SESOI). The R code (RStudio Team, 2016; R Core Team, 2018) below demonstrates how two-way NHST can be performed as well as probability of lower, trivial, and higher effect given the SESOI thresholds.

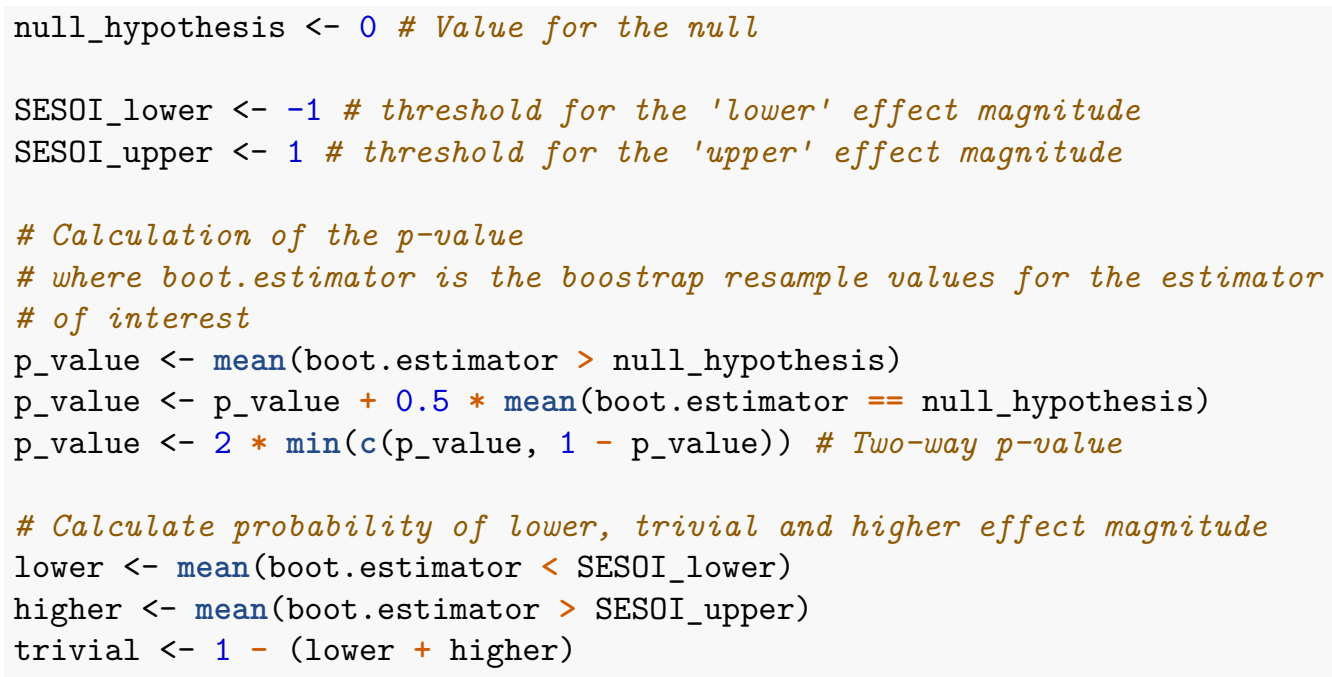

\subsubsection{Bootstrap Type I errors}

As we already did with the frequentist and Bayesian inference, let's get estimates of Type I errors for bootstrap method (10,000 percentile bootstrap resamples) by drawing 1000 samples of $\mathrm{N}=20$ observations from the population where the true mean height is equal to $177.8 \mathrm{~cm}$ and SD is equal to $10.16 \mathrm{~cm}$. Besides estimating Type I error for the sample mean, we can also estimate Type I errors for sample SD and prop185, since the true population values are known. In the case of prop185, the true population value is equal to 0.24 . Type I error is committed when the the $95 \%$ bootstrap CIs of the sample estimate don't cross the true value in the population. Figure 44 depicts the first 100 samples out of the total of 1,000, taken from the population 
with calculated $95 \%$ bootstrap CIs. CIs that missed the true population parameter value are depicted in red. Table 46 contains the summary for this simulation.
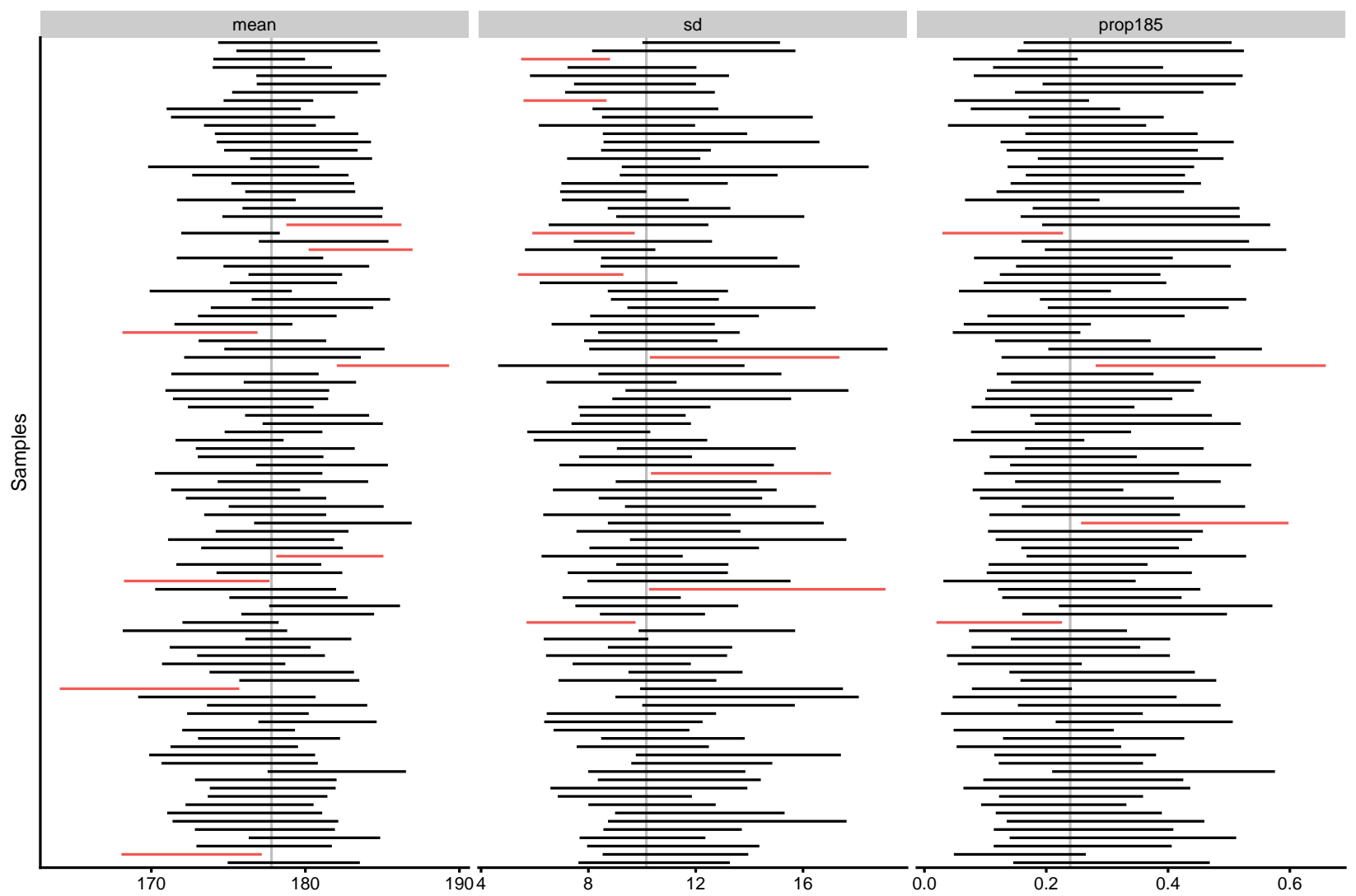

Figure 44. Bootstrap 95\%confidence intervals. Intervals not capturing the true population parameter are colored in red

Table 46. Bootstrap Type I errors

\begin{tabular}{lrrr}
\hline parameter & Sample & Correct \% & Type I Errors \% \\
\hline mean & 1000 & 93.1 & 6.9 \\
sd & 1000 & 88.3 & 11.7 \\
prop185 & 1000 & 92.8 & 7.2 \\
\hline
\end{tabular}

As can be seen from the Table 46, Type I error for the $\sigma$ parameter is larger than expected. This could be due to the non-symmetrical bootstrap distribution that might not be perfectly represented with the percentile approach.

I am not hiding my preference for the bootstrap methods due to their intuitive nature and simple usage for generating inferences for any estimator.

However, bootstrap is not panacea and there are caveats especially for the small samples sizes (Wilcox \& Rousselet, 2017; Wilcox, Peterson \& McNitt-Gray, 2018; Rousselet, Pernet \& Wilcox, 2019b,a). 


\subsection{Statistical inference conclusion}

Which one is better, frequentist, Bayesian or bootstrap? Unfortunately, there is no objective, automatic way to statistical inference. Both demand careful planning and consideration of the research question of interest. Both rely on assumptions, some more clearly stated as with Bayesian, and some more inherent as with frequentist. Both represent Small Worlds that we hope to help explain and to be deployed to the Large World. Rather than approaching inferential statistics as something that represents and estimates true state of the World, inferential statistics might be approached as descriptive statistic (Amrhein, Trafimow \& Greenland, 2019).

The three quotes to summarize this very topic are the following ones:

"Statistical inference often fails to replicate. One reason is that many results may be selected for drawing inference because some threshold of a statistic like the P-value was crossed, leading to biased reported effect sizes. Nonetheless, considerable non-replication is to be expected even without selective reporting, and generalizations from single studies are rarely if ever warranted. Honestly reported results must vary from replication to replication because of varying assumption violations and random variation; excessive agreement itself would suggest deeper problems, such as failure to publish results in conflict with group expectations or desires. A general perception of a "replication crisis" may thus reflect failure to recognize that statistical tests not only test hypotheses, but countless assumptions and the entire environment in which research takes place. Because of all the uncertain and unknown assumptions that underpin statistical inferences, we should treat inferential statistics as highly unstable local descriptions of relations between assumptions and data, rather than as providing generalizable inferences about hypotheses or models. And that means we should treat statistical results as being much more incomplete and uncertain than is currently the norm. Acknowledging this uncertainty could help reduce the allure of selective reporting: Since a small P-value could be large in a replication study, and a large $\mathrm{P}$-value could be small, there is simply no need to selectively report studies based on statistical results. Rather than focusing our study reports on uncertain conclusions, we should thus focus on describing accurately how the study was conducted, what problems occurred, what data were obtained, what analysis methods were used and why, and what output those methods produced." (Amrhein, Trafimow \& Greenland, 2019), Abstract, p. 262

"Exercising awareness of multiple perspectives, we emphasize that we do not believe that one of these philosophies is the correct or best one, nor do we claim that reducing the different approaches to a single one would be desirable. What is lacking here is not unification, but rather, often, transparency about which interpretation of probabilistic outcomes is intended when applying statistical modeling to specific problems. Particularly, we think that, depending on the situation, both "aleatory" or "epistemic" approaches to modeling uncertainty are legitimate and worthwhile, referring to data generating processes in observer-independent reality on one hand and rational degrees of belief on the other." (Gelman \& Hennig, 2017), p. 20

"Broadly speaking, 19th century statistics was Bayesian while the 20th century was frequentist, at least from the point of view of most scientific practitioners. Here in the 21st century scientists are bringing statisticians much bigger problems to solve, often comprising millions of data points and thousands of parameters. Which statistical philosophy will dominate practice? My guess, backed up with some recent examples, is that a combination of Bayesian and frequentist ideas will be needed to deal with our increasingly intense scientific environment. This will be a challenging period for statisticians, both applied and theoretical, but it also opens the opportunity for a new golden age, rivaling that of Fisher, Neyman, and the other giants of the early 1900's." (Efron, 2005)

This being said, my personal opinion is that more and more sport scientists will consider Bayesian analysis, particularly with the new tools that provide ease of Bayesian model definition and training, as well as visualization of the results. For the benefits of Bayesian over frequentist analysis please see the papers by Kruschke et al. (Kruschke \& Liddell, 2018a,b) as well as suggested reading on Bayesian analysis at the end of 
this paper.

\section{Measurement Error}

Measurement error is involved in all measurements and causes an observed score to be different from the true score (Novick, 1966; Allen \& Yen, 2001; Swinton et al., 2018), which affect not only outcome variables and predictors, but also treatment variables. This results in measurement bias affecting both causal inferences (Hernan \& Cole, 2009; Hernán, 2017; Hernán \& Robins) and predictive performances (Kuhn \& Johnson, 2018).

In mathematical notation, we state that the observed score (OS) comprises a hypothetical true score (TS) and measurement error (ME) (equation 41) (Allen \& Yen, 2001; Swinton et al., 2018).

$$
O S=T S+M E
$$

In the sports science domain, since the measured objects are usually humans, measurement error comprises of instrumentation and biological noise (Swinton et al., 2018). In this paper I assume instrumentation noise as error caused solely by the measurement apparatus (Swinton et al., 2018). Biological noise, on the other hand, is defined as an error in the observed scores caused by biological processes, including, but not limited to, phenomena such as circadian rhythm, nutritional intake, sleep and motivation (Swinton et al., 2018).

Both instrumentation and biological noises consist of two types of errors: systematic error and random error (Figure 45). 


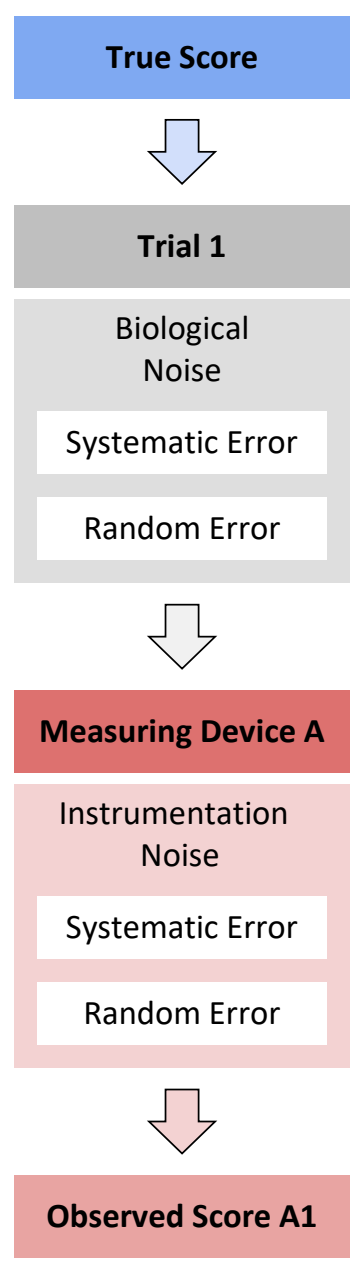

Figure 45. Measurement error

Systematic error represents constant and stable error that is fixed across measurement samples of the true score. Systematic error is commonly refereed to as bias. In instruments having a linear response, systematic error can be further divided into proportional bias and fixed bias (Hopkins, 2004a, 2007, 2010). Random error $(\epsilon)$ represents unknown and unpredictable error, which varies between measurement samples of the same true score. Random errors often have a Gaussian normal distribution (with mean zero and standard deviation which represent a parameter of the random error). The following equation (42) represent theoretical linear relationship between TS and OS, with normally distributed random error:

$$
O S=\text { Fixed Bias }+(\text { Proportional Bias } \times T S)+\epsilon
$$

This can be easily explained with a simple example. Imagine $\mathrm{N}=20$ athletes being measured on a novel bodyweight scale, using 5 trials separated by 1 minute. The assumption in this study is that there is no change in the TS across trials (e.g. athletes are not allowed to pee, consume water or food, nor change the wardrobe) and that there is no biological noise involved (i.e. there are no fluctuations in bodyweight due motivation, fatigue or what have you). Since this is a simulation, we know the TS of the athletes, but also the instrumentation noise characteristic (i.e. DGP) of the novel scale. This scale tends to have proportional bias equal to 1.01 factor (i.e. athlete weighting $100 \mathrm{~kg}$ which is his TS, will have OS equal to $101 \mathrm{~kg}$ due proportional bias, while the athlete weighting $50 \mathrm{~kg}$ will have her OS equal to $50.5 \mathrm{~kg}$ ), fixed bias equal to 
$1 \mathrm{~kg}$ (everyone will get OS higher for $1 \mathrm{~kg}$ than TS), and random error normally distributed with SD equal to $0.5 \mathrm{~kg}$. Equation (43) captures this relationship between OS and TS.

$$
O S_{i}=1+\left(1.01 \times T S_{i}\right)+\mathcal{N}(0,0.5)
$$

Table 47 contains the simulated sample.

Table 47. Simulated 5 trials from known true score and measurement error

\begin{tabular}{lrrrrrr}
\hline Athlete & TS $(\mathrm{kg})$ & OS 1 $(\mathrm{kg})$ & OS 2 $(\mathrm{kg})$ & OS 3 $(\mathrm{kg})$ & OS $4(\mathrm{~kg})$ & OS 5 $(\mathrm{kg})$ \\
\hline Athlete 01 & 77.93 & 79.47 & 79.37 & 79.25 & 80.38 & 79.46 \\
Athlete 02 & 76.11 & 77.83 & 77.71 & 77.36 & 78.32 & 76.78 \\
Athlete 03 & 77.04 & 78.65 & 78.30 & 78.48 & 78.30 & 78.55 \\
Athlete 04 & 54.96 & 56.58 & 56.36 & 56.05 & 56.20 & 56.45 \\
Athlete 05 & 84.03 & 85.53 & 85.44 & 86.10 & 85.96 & 85.11 \\
Athlete 06 & 61.32 & 61.84 & 63.05 & 63.52 & 63.73 & 62.43 \\
Athlete 07 & 68.62 & 70.26 & 69.82 & 70.34 & 70.45 & 70.84 \\
Athlete 08 & 61.06 & 62.03 & 62.91 & 62.93 & 62.15 & 62.40 \\
Athlete 09 & 80.46 & 81.34 & 83.04 & 82.57 & 82.60 & 82.33 \\
Athlete 10 & 91.14 & 94.02 & 93.47 & 93.28 & 93.40 & 93.51 \\
Athlete 11 & 79.98 & 81.89 & 82.13 & 81.13 & 81.57 & 81.94 \\
Athlete 12 & 67.07 & 69.54 & 68.32 & 68.29 & 69.23 & 67.72 \\
Athlete 13 & 79.41 & 80.66 & 80.81 & 81.17 & 80.56 & 80.92 \\
Athlete 14 & 69.54 & 71.14 & 72.34 & 70.16 & 72.01 & 71.11 \\
Athlete 15 & 76.01 & 77.42 & 77.41 & 78.32 & 78.22 & 77.61 \\
Athlete 16 & 68.31 & 70.11 & 70.05 & 70.17 & 69.02 & 70.06 \\
Athlete 17 & 58.53 & 60.04 & 60.56 & 59.69 & 59.72 & 60.69 \\
Athlete 18 & 81.64 & 82.61 & 83.30 & 83.66 & 82.87 & 82.60 \\
Athlete 19 & 55.03 & 56.70 & 56.35 & 56.90 & 56.48 & 56.37 \\
Athlete 20 & 65.03 & 66.33 & 66.44 & 67.29 & 67.28 & 66.82 \\
\hline
\end{tabular}

The objective of the analysis of such data is to estimate DGP parameters of the measurement error, or the proportional bias, fixed bias, and the SD of the random error. Unfortunately, since TS is unknown, we are unable to estimate proportional bias and fixed bias. To overcome this problem, we usually compare OS to some gold standard measure which can serve as proxy to TS. These issues are covered in much more detail in the Validity and Reliability sections of the second part of this paper.

What we are left to estimate is the SD of the random error. For this reason, this is referred to as typical error (TE) of the test, which represents an estimate of a random error SD. This is simply estimated using individual SD of the OS in the five trials (Table 48).

Table 48. Individual mean and SD from five trials 


\begin{tabular}{lcc}
\hline Athlete & Mean & SD \\
\hline Athlete 01 & 79.59 & 0.45 \\
Athlete 02 & 77.60 & 0.57 \\
Athlete 03 & 78.46 & 0.16 \\
Athlete 04 & 56.33 & 0.21 \\
Athlete 05 & 85.63 & 0.40 \\
Athlete 06 & 62.91 & 0.78 \\
Athlete 07 & 70.34 & 0.37 \\
Athlete 08 & 62.48 & 0.42 \\
Athlete 09 & 82.38 & 0.63 \\
Athlete 10 & 93.54 & 0.28 \\
Athlete 11 & 81.73 & 0.39 \\
Athlete 12 & 68.62 & 0.75 \\
Athlete 13 & 80.82 & 0.24 \\
Athlete 14 & 71.35 & 0.86 \\
Athlete 15 & 77.80 & 0.44 \\
Athlete 16 & 69.88 & 0.49 \\
Athlete 17 & 60.14 & 0.47 \\
Athlete 18 & 83.01 & 0.46 \\
Athlete 19 & 56.56 & 0.24 \\
Athlete 20 & 66.83 & 0.45 \\
\hline
\end{tabular}

The mean of athletes' typical errors (SD in table 48 ) is equal to $0.45 \mathrm{~cm}$, which is quite close to DGP parameter of $0.5 \mathrm{~cm}$. The reason for the difference between estimated and true parameter of the random error SD is due to the sampling error, which is a topic already covered in the "Statistical inference" section of this paper.

Unfortunately, this method of estimating random error SD is not always practically feasible, and we usually estimate SD using two trials (Table 49), where SD of random error is estimated using SD of the difference scores across athletes (Hopkins, 2000; Swinton et al., 2018).

Table 49. Estimating Typical Error using SD of the difference scores 


\begin{tabular}{lrrr}
\hline Athlete & OS 1 $(\mathrm{kg})$ & OS 2 $(\mathrm{kg})$ & Difference OS 2-1 $(\mathrm{kg})$ \\
\hline Athlete 01 & 79.47 & 79.37 & -0.11 \\
Athlete 02 & 77.83 & 77.71 & -0.11 \\
Athlete 03 & 78.65 & 78.30 & -0.35 \\
Athlete 04 & 56.58 & 56.36 & -0.23 \\
Athlete 05 & 85.53 & 85.44 & -0.09 \\
Athlete 06 & 61.84 & 63.05 & 1.22 \\
Athlete 07 & 70.26 & 69.82 & -0.44 \\
Athlete 08 & 62.03 & 62.91 & 0.88 \\
Athlete 09 & 81.34 & 83.04 & 1.70 \\
Athlete 10 & 94.02 & 93.47 & -0.55 \\
Athlete 11 & 81.89 & 82.13 & 0.24 \\
Athlete 12 & 69.54 & 68.32 & -1.22 \\
Athlete 13 & 80.66 & 80.81 & 0.15 \\
Athlete 14 & 71.14 & 72.34 & 1.21 \\
Athlete 15 & 77.42 & 77.41 & -0.01 \\
Athlete 16 & 70.11 & 70.05 & -0.06 \\
Athlete 17 & 60.04 & 60.56 & 0.52 \\
Athlete 18 & 82.61 & 83.30 & 0.70 \\
Athlete 19 & 56.70 & 56.35 & -0.35 \\
Athlete 20 & 66.33 & 66.44 & 0.11 \\
\hline
\end{tabular}

If we calculate SD of the difference scores from the Table (49), we get $0.7 \mathrm{~cm}$. However, this is not quite right, since we know that the true SD of the random error is $0.5 \mathrm{~cm}$. This happens because random error is affecting both Trial 1 and Trial 2, and is propagated to the difference score (Figure 46). 


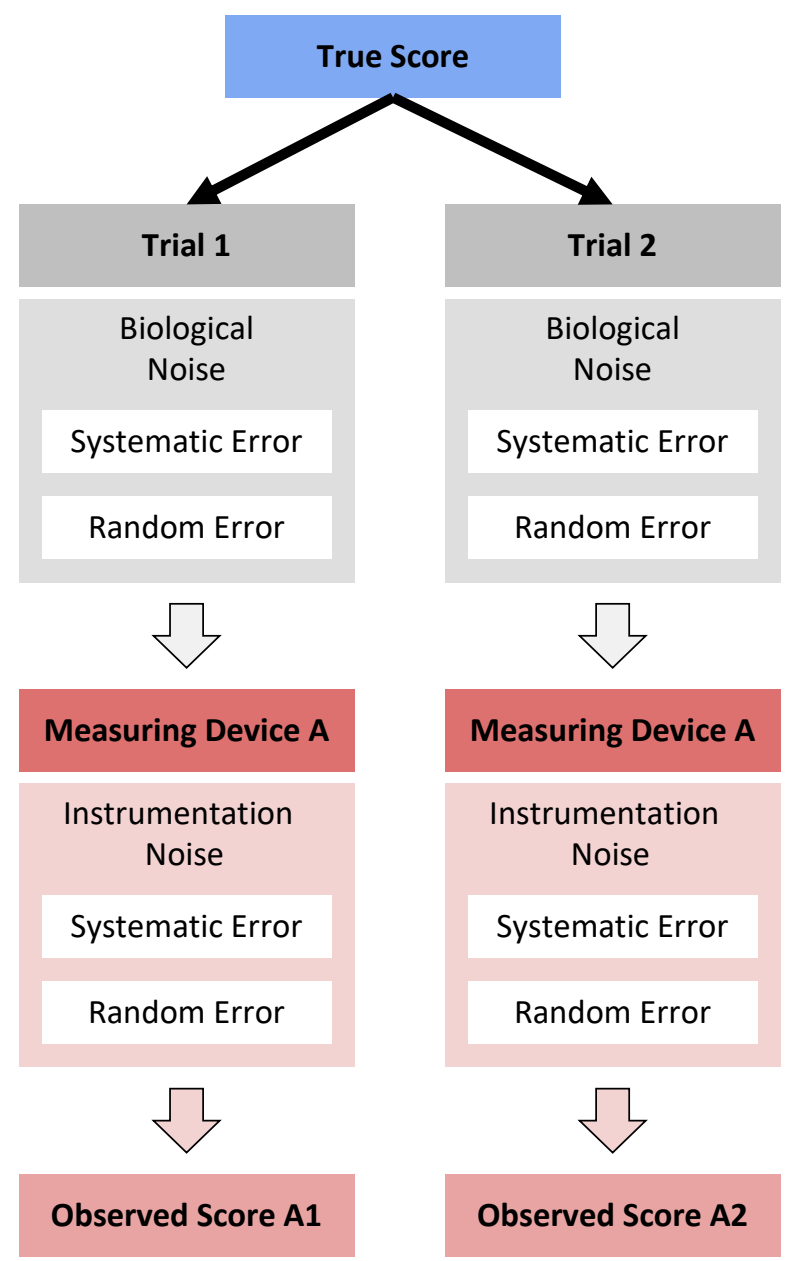

Figure 46. Propagation of the random component of measurement error to two trials

Error propagation, assuming normal distribution, is done quadratically. Mathematically expressed, this is equal to (equation 44):

$$
\begin{aligned}
S D_{\text {difference }}^{2} & =T E_{\text {trial } 1}^{2}+T E_{\text {trial } 2}^{2} \\
T E_{\text {test }} & =T E_{\text {trial } 1}=T E_{\text {trial } 2} \\
S D_{\text {difference }}^{2} & =T E_{\text {test }}^{2}+T E_{\text {test }}^{2} \\
S D_{\text {difference }}^{2} & =2 \times T E_{\text {test }}^{2} \\
S D_{\text {difference }} & =\sqrt{2 \times T E_{\text {test }}^{2}} \\
S D_{\text {difference }} & =\sqrt{2} \times T E_{\text {test }} \\
T E_{\text {test }} & =\frac{S D_{\text {difference }}}{\sqrt{2}}
\end{aligned}
$$

According to the above equation, the SD of the difference score needs to be divided by $\sqrt{2}$ to calculate TE of the test. The assumption is that TE is equal in both trials, which is defined in the second line in equation (44). This way we get TE equal to $0.49 \mathrm{~cm}$, which is much closer to known $\mathrm{SD}$ of the random error of $0.5 \mathrm{~cm}$ 
Estimated TE is very useful metric, since it can be used to estimate the true change of the individuals after intervention, to estimate reliability of the test (Hopkins, 2000, 2004b, 2007, 2010, 2015a; Hopkins et al., 2009), and be useful in defining SESOI as explained in the "Description" section of this paper. Further usage of TE in the reliability and validity analysis is covered in the second part of this paper.

\subsection{Interpreting individual changes using SESOI and TE}

In order to showcase the interpretation of the individual changes by using SESOI and TE (named observed outcome approach in "Responders vs non-responders" section), let's consider bench press example from the "Comparing dependent groups" (Table 8).

Imagine that, before commencing this simple intervention, we have estimated measurement error (using TE estimator) of the bench press $1 \mathrm{RM}$ test, which is equal to $2.5 \mathrm{~kg}$. Practically, this means that due to the biological variation and noise, and instrumentation error (e.g. plates no smaller than $2.5 \mathrm{~kg}$ or $1.25 \mathrm{~kg}$ ), $1 \mathrm{RM}$ in the bench press would tend to vary normally distributed with TE equal to $2.5 \mathrm{~kg}$, given, of course, no real change in the strength.

Since the measurement error is involved in both Pre-test and Post-test, due to the error propagation, TE for the Change score is thus equal to $\sqrt{2} \times T E(3.54 \mathrm{~kg})$.

When interviewing a coach, she told us that she considers $\pm 5 \mathrm{~kg}$ as the minimal important change, which we will use as SESOI. Since we know both TE and SESOI, we might be asking how certain are we that the individual change is practically significant (i.e. lower, trivial, or higher compared to SESOI)? This is because we do not know individual true scores, but only observed scores. TE tells us how much of observed score randomly varies around the true score. The question we are trying to answer is: "how likely individual's true score is within lower, trivial, or higher range, given the known TE?"

Figure 47A depicts individual Change scores probabilistically using the known TE. Using the SESOI as trivial change, we can estimate individual probability of lower, trivial, and higher change. Figure 47B depicts individual change scores with error bars representing $95 \%$ confidence intervals (calculated using $\pm 1.96 \times \sqrt{2} \times T E)$. The numbers in brackets on Figure 47B represent estimated probabilities of the true change score being lower, trivial, and higher compared to SESOI. To be more certain of individual changes, TE needs to be smaller, compared to SESOI. Ratio between SESOI and TE can thus represent an estimate of the test sensitivity to detect practically meaningful changes. The smallest change that has at least $95 \%$ chance of being higher or lower than SESOI is SESOI $\pm 1.96 \times \sqrt{2} \times T E$, or $11.93 \mathrm{~kg}$. Graphically, bench press $1 \mathrm{RM}$ change of $\pm 11.93 \mathrm{~kg}$ is the smallest change, where $95 \%$ confidence intervals do not touch SESOI band. 

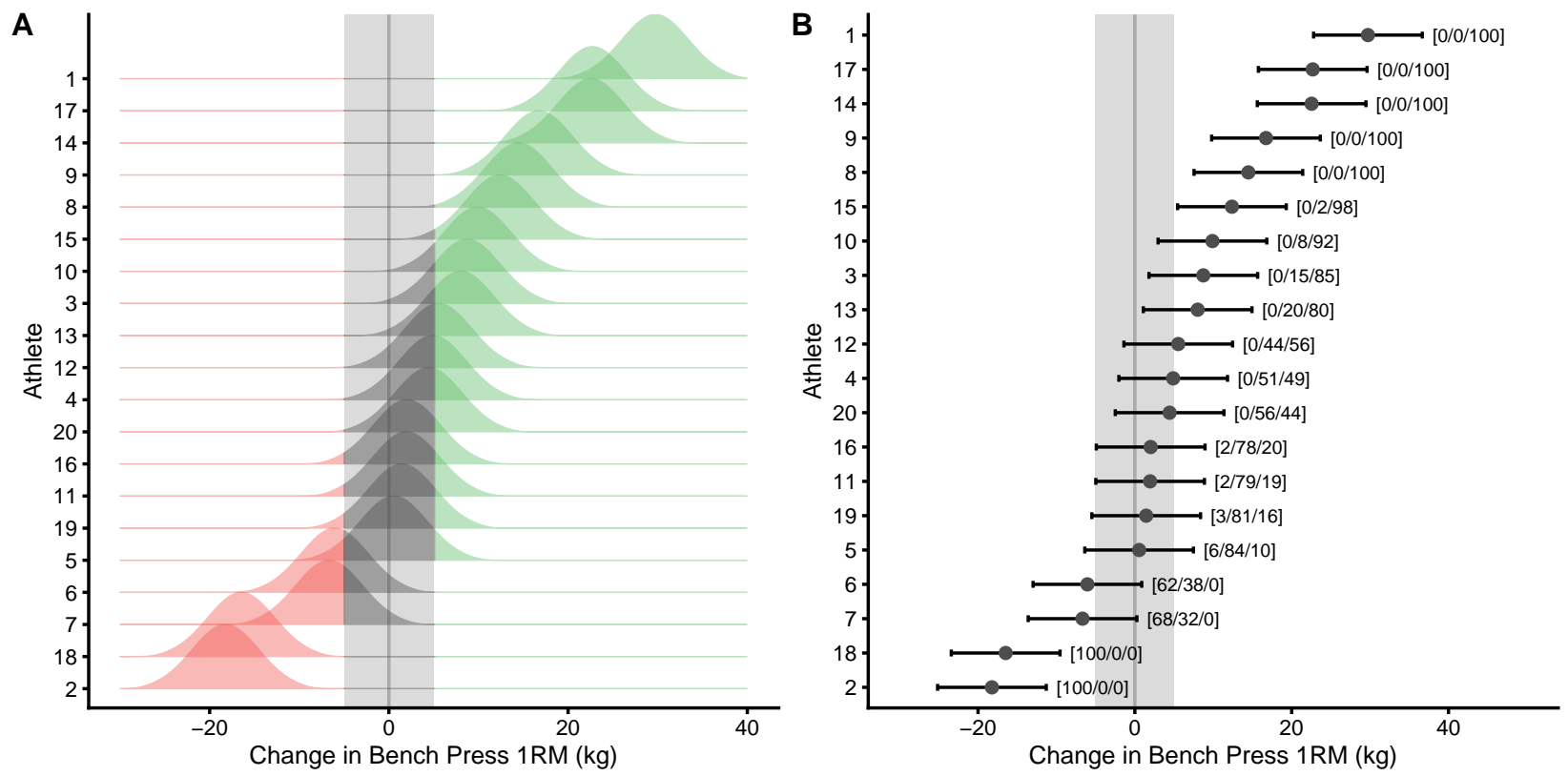

Figure 47. Analysis of individual change scores using SESOI and TE. A. Uncertainty around true score can be depicted by using normal distribution whose SD is equal to TE. Confidence that the observed change is lower, trivial or higher can be estimated using surface within lower, trivia, and higher magnitude band. B. $95 \%$ Confidence intervals around change scores are calculated using $\pm 1.96 \times \sqrt{2} \times T E$. Numbers in brackets represent proportion of the surface area in the lower, trivial and higher magnitude band. These are interpreted as probabilities of true score being in lower, trivial and higher magnitude band. See text for discussion why such interpretation is not statistically valid

As explained in the "Statistical inference" section of this paper, the above method of individual analysis interprets the TE and associated confidence intervals from the Bayesian perspective. This might not be the appropriate interpretation, since we do not know individual's true scores, only the observed scores. TE gives us the variance of the observed scores around the true score, not vice versa (i.e. Bayesian inverse probability). Thus, visual representation from the Figure 47 is not statistically valid, although the inferences are.

Since we do not know the true scores, we are interested in probabilities of seeing the observed score given the assumption of where we think the true score is. For this reason the question to be asked is "assuming individual's true change scores are at \pm SESOI, how likely are we to see the observed score, given the known TE?". This question demands answer and interpretation of TE from the frequentist perspective. Thus the correction interpretation of the individual changes involves the use of minimum effect tests (METs) discussed in the "Statistical Inference" section. METs approach to interpreting individual changes is depicted in the Figure 48. 

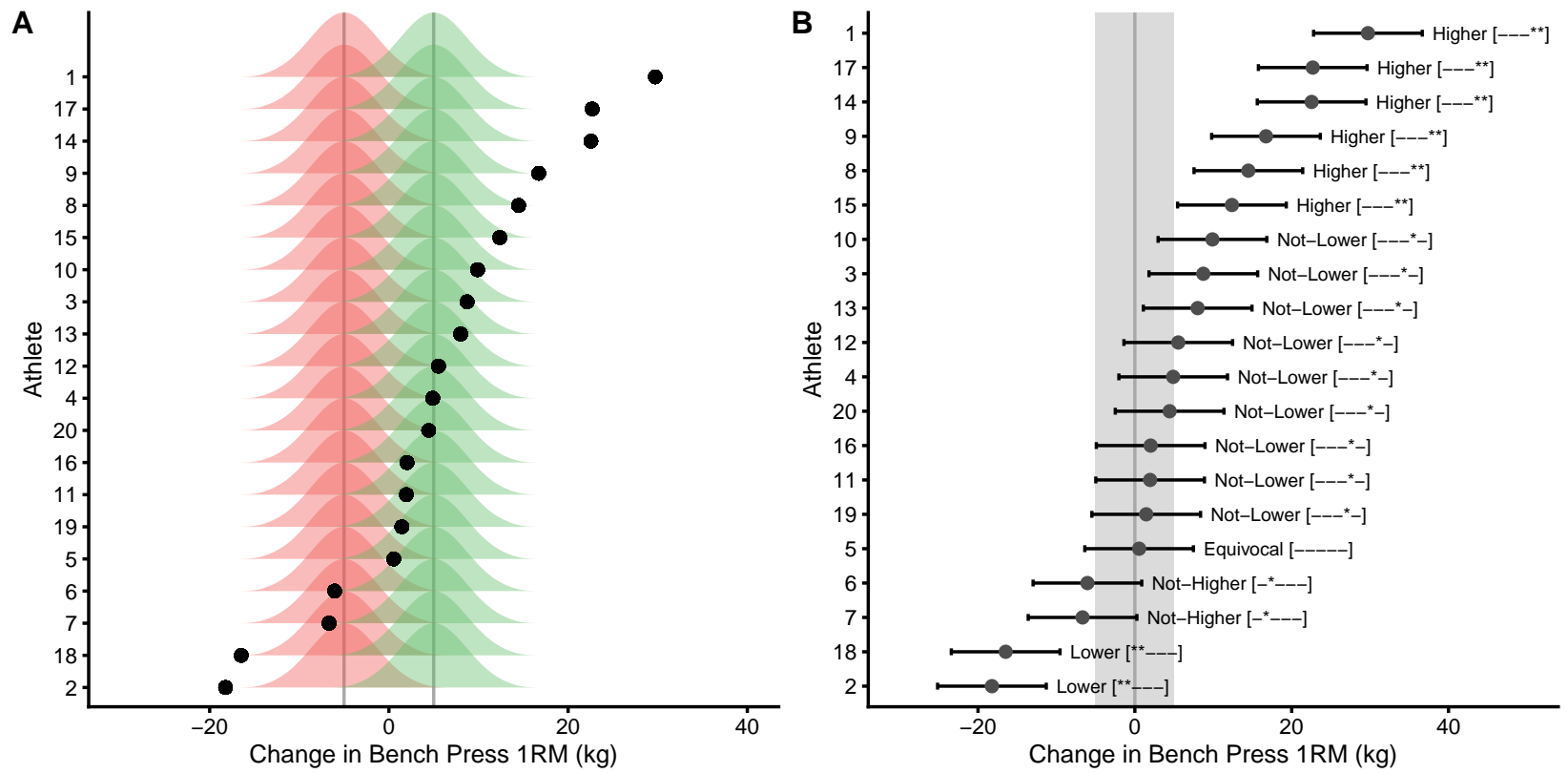

Figure 48. METs approach to interpreting individual change scores. A. Since true change scores are unknown, we can only test probability of seeing observed score or higher, given the known TE and assumed true score. In order to do this, minimal effect tests are performed, assuming two true score null-hypotheses: one at lower SESOI threshold (red color) and one at higher SESOI threshold (green color). Typical error can be interpreted as SD of the error distribution. Black dots indicate observed individual change. We are interested in estimating probability of observing this change, given two hypotheses. Five tests are performed as described in "Minimum Effect Tests" section: inferiority, non-superiority, equivalence, non-inferiority and superiority. B. 95\% Confidence intervals around change scores are calculated using $\pm 1.96 \times \sqrt{2} \times T E$ and depicted using error-bars. Final inference using five METs is reported. METs significance (assuming alpha $=0.05$ ), indicated by ${ }^{*}$, , are reported in the brackets

\section{Conclusion}

Statistical analysis should start with questions that we are trying to answer using the data. These questions of interest should not only guide statistical analysis, but also as data collection and finally interpretations of the analysis and modelling results. In order to answer these questions with data, we are always representing the Large World with the Small World models. There is no entirely objective approach to do it, rather apply pluralism of approaches (Mitchell, 2002, 2012). The value of these models and Small World representations should be judged by qualities suggested by Gelman and Hennig (Gelman \& Hennig, 2017): transparency, consensus, impartiality, correspondence to observable reality, awareness of multiple perspectives, awareness of context-dependence, and investigation of stability. Finally, we need to accept that we must act based on cumulative knowledge rather than solely rely on single studies or even single lines of research (Amrhein, Trafimow \& Greenland, 2019).

For example, let's take a question that a sport practitioner might ask: "From how many athletes I can expect to show positive improvements after this intervention? Will Johnny improve?". This question is more predictive question than it is common in practice. Assume that I provide this coach with the estimate of the average causal effect and accompanying magnitude-based inference (MBI), using SESOI he provided (i.e. $20 \%$ harmful, $30 \%$ trivial, and $50 \%$ beneficial) or frequentist $\mathrm{p} \_$value of $\mathrm{p}<0.05$. Will that answer the practitioner's question? 
The accompanying MBIs might even confuse him and appear to answer the "proportion" question he asked. "So, $50 \%$ of athletes will show beneficial response to treatment?". Unfortunately no - MBIs (or METs) answer different question about estimator (be it mean or Cohen's d or some other), not about individual response proportions.

Second part of his question also demands predictive modelling, that calls for taking into account Johnny's known data and getting the best estimate for his response. If there is some historical data about Johnny, we might get better predictions (either through individual modeling or hierarchical model), but if not, then the average-based estimators might be our best guess of the most likely response that Johnny might manifest. Providing that proportions of responses on top of the average effect might help answering uncertainty about individual responses. Providing that CIs and p-values might not.

I am not saying that these are not important. I am only saying that they should not be automatically selected as an answer to any question. CIs can provide us with the uncertainty interval around proportions (i.e. "Model gives us $90 \%$ confidence that the proportion of the beneficial responses will be $40-60 \%$ "), or even around predictive performance metrics. However, we need to make sure to start with the question asked, as well as to suit our analysis and conclusions so that practitioners can understand it.

In the following part of this paper, I will provide solution to the most common sport science problems and question using the material covered in this part, as well as bootstrap magnitude based predictions (bmbp) package written by the author.

\section{Recommended material}

\section{General}

1. Spiegelhalter D. 2019. The art of statistics: how to learn from data. New York: Basic Books, an imprint of Perseus Books, a subsidiary of Hachette Book Group.

2. Foreman JW. 2014. Data smart: using data science to transform information into insight. Hoboken, New Jersey: John Wiley \& Sons.

3. Dienes Z. 2008. Understanding Psychology as a Science: An Introduction to Scientific and Statistical Inference. New York: Red Globe Press.

\section{Bayesian analysis}

4. Kruschke JK. 2015. Doing Bayesian data analysis: a tutorial with R, JAGS, and Stan. Boston: Academic Press.

5. McElreath R. 2015. Statistical Rethinking: A Bayesian Course with Examples in R and Stan. Boca Raton: Chapman and Hall/CRC.

6. Lambert B. 2018. A student's guide to Bayesian statistics. Los Angeles: SAGE.

7. Stanton JM. 2017. Reasoning with data: an introduction to traditional and Bayesian statistics using R. New York: The Guilford Press.

\section{Predictive analysis and Machine Learning}

8. Kuhn M, Johnson K. 2018. Applied Predictive Modeling. New York: Springer.

9. Kuhn M, Johnson K. 2019. Feature Engineering and Selection: a Practical Approach for Predictive Models. Milton: CRC Press LLC.

10. Lantz B. 2019. Machine learning with R: expert techniques for predictive modeling. 
11. James G, Witten D, Hastie T, Tibshirani R. 2017. An Introduction to Statistical Learning: with Applications in R. New York: Springer.

\section{Causal inference}

12. Kleinberg S. 2015. Why: A Guide to Finding and Using Causes. Beijing; Boston: O'Reilly Media.

13. Pearl J, Mackenzie D. 2018. The Book of Why: The New Science of Cause and Effect. New York: Basic Books.

14. Hernán MA, Robins J. 2019. Causal Inference. Boca Raton: Chapman \& Hall/CRC.

\section{R Language and Visualization}

15. Kabacoff R. 2015. R in action: data analysis and graphics with R. Shelter Island: Manning.

16. Matloff NS. 2011. The art of R programming: tour of statistical software design. San Francisco: No Starch Press.

17. Wickham H, Grolemund G. 2016. R for data science: import, tidy, transform, visualize, and model data. Sebastopol, CA: O'Reilly.

18. Wilke C. 2019. Fundamentals of data visualization: a primer on making informative and compelling figures. Sebastopol, CA: O'Reilly Media.

19. Healy K. 2018. Data visualization: a practical introduction. Princeton, NJ: Princeton University Press.

\section{Simulation and statistical inference}

20. Carsey T, Harden J. 2013. Monte Carlo Simulation and Resampling Methods for Social Science. Los Angeles: Sage Publications, Inc.

21. Efron B, Hastie T. 2016. Computer Age Statistical Inference: Algorithms, Evidence, and Data Science. New York, NY: Cambridge University Press.

Online Courses 22. Hastie T, Tibshirani R. 2016.Statistical Learning. Available at https://lagunita.stanford. edu/courses/HumanitiesSciences/StatLearning/Winter2016/about (accessed October 16, 2019).

23. Lakens D. 2017.Improving your statistical inferences. Available at https://www.coursera.org/learn/ statistical-inferences (accessed October 16, 2019).

24. Lakens D. 2019.Improving your statistical questions. Available at https://www.coursera.org/learn/ improving-statistical-questions (accessed October 16, 2019).

\section{References}

Albanese D, Filosi M, Visintainer R, Riccadonna S, Jurman G, Furlanello C. 2012. Minerva and minepy: A $\mathrm{C}$ engine for the MINE suite and its R, Python and MATLAB wrappers. Bioinformatics:bts707.

Allen M, Poggiali D, Whitaker K, Marshall TR, Kievit R. 2018. Raincloudplots Tutorials And Codebase. DOI: 10.5281/zenodo.1402959.

Allen M, Poggiali D, Whitaker K, Marshall TR, Kievit RA. 2019. Raincloud plots: A multi-platform tool for robust data visualization. Wellcome Open Research 4:63. DOI: 10.12688/wellcomeopenres.15191.1.

Allen MJ, Yen WM. 2001. Introduction to Measurement Theory. Long Grove, Ill: Waveland Pr Inc. 
Amrhein V, Trafimow D, Greenland S. 2019. Inferential Statistics as Descriptive Statistics: There Is No Replication Crisis if We Don't Expect Replication. The American Statistician 73:262-270. DOI: 10.1080/00031305.2018.1543137.

Angrist JD, Pischke J-S. 2015. Mastering 'metrics: The path from cause to effect. Princeton ; Oxford: Princeton University Press.

Anvari F, Lakens D. 2019. Using Anchor-Based Methods to Determine the Smallest Effect Size of Interest. DOI: $10.31234 /$ osf.io/syp5a.

Barker RJ, R. Schofield M. 2008. Inference About Magnitudes of Effects. International Journal of Sports Physiology and Performance 3:547-557. DOI: 10.1123/ijspp.3.4.547.

Batterham AM, Hopkins WG. 2006. Making Meaningful Inferences About Magnitudes. International Journal of Sports Physiology and Performance 1:50-57. DOI: 10.1123/ijspp.1.1.50.

Beaujean AA. 2014. Latent variable modeling using R: A step by step guide. New York: Routledge/Taylor \& Francis Group.

Binmore K. 2011. Rational Decisions. Princeton, NJ: Princeton University Press.

Borg DN, Minett GM, Stewart IB, Drovandi CC. 2018. Bayesian Methods Might Solve the Problems with Magnitude-based Inference: Medicine $\&$ Science in Sports $\&$ Exercise 50:2609-2610. DOI: 10.1249/MSS.0000000000001736.

Borsboom D. 2008. Latent Variable Theory. Measurement: Interdisciplinary Research \& Perspective 6:25-53. DOI: $10.1080 / 15366360802035497$.

Borsboom D, Mellenbergh GJ, van Heerden J. 2003. The theoretical status of latent variables. Psychological Review 110:203-219. DOI: 10.1037/0033-295X.110.2.203.

Breiman L. 2001. Statistical Modeling: The Two Cultures. Statistical Science 16:199-215.

Buchheit M, Rabbani A. 2014. The 3015 Intermittent Fitness Test Versus the Yo-Yo Intermittent Recovery Test Level 1: Relationship and Sensitivity to Training. International Journal of Sports Physiology and Performance 9:522-524. DOI: 10.1123/ijspp.2012-0335.

Caldwell AR, Cheuvront SN. 2019. Basic statistical considerations for physiology: The journal Temperature toolbox. Temperature:1-30. DOI: 10.1080/23328940.2019.1624131.

Canty A, Ripley BD. 2017. Boot: Bootstrap R (S-Plus) Functions.

Carsey T, Harden J. 2013. Monte Carlo Simulation and Resampling Methods for Social Science. Los Angeles: Sage Publications, Inc.

Cohen J. 1988. Statistical power analysis for the behavioral sciences. Hillsdale, N.J: L. Erlbaum Associates.

Cumming G. 2014. The New Statistics: Why and How. Psychological Science 25:7-29. DOI: $10.1177 / 0956797613504966$.

Curran-Everett D. 2018. Magnitude-based Inference: Good Idea but Flawed Approach. Medicine E3 Science in Sports 8 Exercise 50:2164-2165. DOI: 10.1249/MSS.0000000000001646.

Davison AC, Hinkley DV. 1997. Bootstrap Methods and Their Applications. Cambridge: Cambridge University Press.

Dienes Z. 2008. Understanding Psychology as a Science: An Introduction to Scientific and Statistical Inference. New York: Red Globe Press.

Efron B. 2005. Bayesians, Frequentists, and Scientists. Journal of the American Statistical Association 100:1-5. DOI: 10.1198/016214505000000033.

Efron B, Hastie T. 2016. Computer Age Statistical Inference: Algorithms, Evidence, and Data Science. New York, NY: Cambridge University Press. 
Estrada E, Ferrer E, Pardo A. 2019. Statistics for Evaluating Pre-post Change: Relation Between Change in the Distribution Center and Change in the Individual Scores. Frontiers in Psychology 9. DOI: 10.3389/fpsyg.2018.02696.

Everitt B, Hothorn T. 2011. An introduction to applied multivariate analysis with R. New York: Springer.

Finch WH, French BF. 2015. Latent variable modeling with R. New York: Routledge, Taylor \& Francis Group.

Fisher AJ, Medaglia JD, Jeronimus BF. 2018. Lack of group-to-individual generalizability is a threat to human subjects research. Proceedings of the National Academy of Sciences 115:E6106-E6115. DOI: $10.1073 /$ pnas. 1711978115.

Foreman JW. 2014. Data smart: Using data science to transform information into insight. Hoboken, New Jersey: John Wiley \& Sons.

Friedman J, Hastie T, Tibshirani R. 2010. Regularization paths for generalized linear models via coordinate descent. Journal of Statistical Software 33:1-22.

Gelman A. 2011. Causality and Statistical Learning. American Journal of Sociology 117:955-966. DOI: $10.1086 / 662659$.

Gelman A, Greenland S. 2019. Are confidence intervals better termed "uncertainty intervals"? BMJ:15381. DOI: $10.1136 / \mathrm{bmj} .15381$.

Gelman A, Hennig C. 2017. Beyond subjective and objective in statistics. Journal of the Royal Statistical Society: Series A (Statistics in Society) 180:967-1033. DOI: 10.1111/rssa.12276.

Gigerenzer G, Hertwig R, Pachur T. 2015. Heuristics: The Foundations of Adaptive Behavior. Oxford University Press.

Glazier PS, Mehdizadeh S. 2018. Challenging Conventional Paradigms in Applied Sports Biomechanics Research. Sports Medicine. DOI: 10.1007/s40279-018-1030-1.

Goldstein A, Kapelner A, Bleich J, Pitkin E. 2013. Peeking Inside the Black Box: Visualizing Statistical Learning with Plots of Individual Conditional Expectation. arXiv:1309.6392 [stat].

Hastie T, Tibshirani R, Friedman JH. 2009. The elements of statistical learning: Data mining, inference, and prediction. New York, NY: Springer.

Heckman JJ. 2005. Rejoinder: Response to Sobel. Sociological Methodology 35:135-150. DOI: 10.1111/j.00811750.2006.00166.x.

Hecksteden A, Kraushaar J, Scharhag-Rosenberger F, Theisen D, Senn S, Meyer T. 2015. Individual response to exercise training - a statistical perspective. Journal of Applied Physiology 118:1450-1459. DOI: 10.1152/japplphysiol.00714.2014.

Hecksteden A, Pitsch W, Rosenberger F, Meyer T. 2018. Repeated testing for the assessment of individual response to exercise training. Journal of Applied Physiology 124:1567-1579. DOI: 10.1152/japplphysiol.00896.2017.

Henry L, Wickham H, Chang W. 2019. Ggstance: Horizontal 'ggplot2' Components.

Hernán MA. 2016. Does water kill? A call for less casual causal inferences. Annals of epidemiology 26:674-680. DOI: 10.1016/j.annepidem.2016.08.016.

Hernán MA. 2017. Causal Diagrams: Draw Your Assumptions Before Your Conclusions Course | PH559x | edX. edX.

Hernán MA. 2018. The C-Word: Scientific Euphemisms Do Not Improve Causal Inference From Observational Data. American Journal of Public Health 108:616-619. DOI: 10.2105/AJPH.2018.304337.

Hernan MA. 2002. Causal Knowledge as a Prerequisite for Confounding Evaluation: An Application to Birth Defects Epidemiology. American Journal of Epidemiology 155:176-184. DOI: 10.1093/aje/155.2.176. 
Hernan MA, Cole SR. 2009. Invited Commentary: Causal Diagrams and Measurement Bias. American Journal of Epidemiology 170:959-962. DOI: 10.1093/aje/kwp293.

Hernán MA, Hsu J, Healy B. 2019. A Second Chance to Get Causal Inference Right: A Classification of Data Science Tasks. CHANCE 32:42-49. DOI: 10.1080/09332480.2019.1579578.

Hernán MA, Robins J. Causal Inference. Boca Raton: Chapman \& Hall/CRC.

Hernán MA, Taubman SL. 2008. Does obesity shorten life? The importance of well-defined interventions to answer causal questions. International Journal of Obesity 32:S8-S14. DOI: 10.1038/ijo.2008.82.

Hesterberg TC. 2015. What Teachers Should Know About the Bootstrap: Resampling in the Undergraduate Statistics Curriculum. The American Statistician 69:371-386. DOI: 10.1080/00031305.2015.1089789.

Hocking TD. 2018. Directlabels: Direct Labels for Multicolor Plots.

Hopkins WG. 2000. Measures of Reliability in Sports Medicine and Science. Sports Med:15.

Hopkins WG. 2004a. Bias in Bland-Altman but not Regression Validity Analyses. Sportscience.org.

Hopkins WG. 2004b. How to Interpret Changes in an Athletic Performance Test.:2.

Hopkins WG. 2006. New View of Statistics: Effect Magnitudes.

Hopkins WG. 2007. Understanding Statistics by Using Spreadsheets to Generate and Analyze Samples. Sportscience.org.

Hopkins WG. 2010. A Socratic Dialogue on Comparison of Measures. Sportscience.org.

Hopkins W. 2015a. Spreadsheets for analysis of validity and reliability. Sportscience.org:9.

Hopkins WG. 2015b. Individual responses made easy. Journal of Applied Physiology 118:1444-1446. DOI: 10.1152/japplphysiol.00098.2015.

Hopkins W, Batterham A. 2018. The Vindication of Magnitude-Based Inference.:12.

Hopkins WG, Marshall SW, Batterham AM, Hanin J. 2009. Progressive Statistics for Studies in Sports Medicine and Exercise Science: Medicine 83 Science in Sports \& Exercise 41:3-13. DOI: 10.1249/MSS.0b013e31818cb278.

James G, Witten D, Hastie T, Tibshirani R. 2017. An Introduction to Statistical Learning: With Applications in $R$. New York: Springer.

Kabacoff R. 2015. $R$ in action: Data analysis and graphics with $R$. Shelter Island: Manning.

King MT. 2011. A point of minimal important difference (MID): A critique of terminology and methods. Expert Review of Pharmacoeconomics 83 Outcomes Research 11:171-184. DOI: 10.1586/erp.11.9.

Kleinberg S. 2015. Why: A Guide to Finding and Using Causes. Beijing ; Boston: O'Reilly Media.

Kleinberg S. 2018. Causality, probability, and time.

Kleinberg J, Liang A, Mullainathan S. 2017. The Theory is Predictive, but is it Complete? An Application to Human Perception of Randomness. arXiv:1706.06974 [cs, stat].

Kruschke JK. 2013. Bayesian estimation supersedes the t test. Journal of Experimental Psychology: General 142:573-603. DOI: 10.1037/a0029146.

Kruschke JK, Liddell TM. 2018a. Bayesian data analysis for newcomers. Psychonomic Bulletin 8 Review 25:155-177. DOI: 10.3758/s13423-017-1272-1.

Kruschke JK, Liddell TM. 2018b. The Bayesian New Statistics: Hypothesis testing, estimation, metaanalysis, and power analysis from a Bayesian perspective. Psychonomic Bulletin 63 Review 25:178-206. DOI: 10.3758/s13423-016-1221-4.

Kuhn M, Johnson K. 2018. Applied Predictive Modeling. New York: Springer. 
Kuhn M, Johnson K. 2019. Feature Engineering and Selection: A Practical Approach for Predictive Models. Milton: CRC Press LLC.

Kuhn M, Wing J, Weston S, Williams A, Keefer C, Engelhardt A, Cooper T, Mayer Z, Kenkel B, R. Core Team, Benesty M, Lescarbeau R, Ziem A, Scrucca L, Tang Y, Candan C. 2018. Caret: Classification and Regression Training.

Lakens D. 2017. Equivalence Tests: A Practical Primer for $t$ Tests, Correlations, and Meta-Analyses. Social Psychological and Personality Science 8:355-362. DOI: 10.1177/1948550617697177.

Lakens D, Scheel AM, Isager PM. 2018. Equivalence Testing for Psychological Research: A Tutorial. Advances in Methods and Practices in Psychological Science 1:259-269. DOI: 10.1177/2515245918770963.

Lang KM, Sweet SJ, Grandfield EM. 2017. Getting beyond the Null: Statistical Modeling as an Alternative Framework for Inference in Developmental Science. Research in Human Development 14:287-304. DOI: 10.1080/15427609.2017.1371567.

Lederer DJ, Bell SC, Branson RD, Chalmers JD, Marshall R, Maslove DM, Ost DE, Punjabi NM, Schatz M, Smyth AR, Stewart PW, Suissa S, Adjei AA, Akdis CA, Azoulay, Bakker J, Ballas ZK, Bardin PG, Barreiro E, Bellomo R, Bernstein JA, Brusasco V, Buchman TG, Chokroverty S, Collop NA, Crapo JD, Fitzgerald DA, Hale L, Hart N, Herth FJ, Iwashyna TJ, Jenkins G, Kolb M, Marks GB, Mazzone P, Moorman JR, Murphy TM, Noah TL, Reynolds P, Riemann D, Russell RE, Sheikh A, Sotgiu G, Swenson ER, Szczesniak R, Szymusiak R, Teboul J-L, Vincent J-L. 2019. Control of Confounding and Reporting of Results in Causal Inference Studies. Guidance for Authors from Editors of Respiratory, Sleep, and Critical Care Journals. Annals of the American Thoracic Society 16:22-28. DOI: 10.1513/AnnalsATS.201808-564PS.

Makowski D, Ben-Shachar MS, Chen SA, Lüdecke D. Indices of Effect Existence and Significance in the Bayesian Framework. DOI: 10.31234/osf.io/2zexr.

Makowski D, Ben-Shachar MS, Lüdecke D. 2019a. Understand and describe bayesian models and posterior distributions using bayestestR. CRAN. DOI: 10.5281/zenodo.2556486.

Makowski D, Ben-Shachar M, Lüdecke D. 2019b. bayestestR: Describing Effects and their Uncertainty, Existence and Significance within the Bayesian Framework. Journal of Open Source Software 4:1541. DOI: 10.21105/joss.01541.

McElreath R. 2015. Statistical Rethinking: A Bayesian Course with Examples in $R$ and Stan. Boca Raton: Chapman and Hall/CRC.

McGraw KO, Wong SP. 1992. A common language effect size statistic. Psychological Bulletin 111:361-365. DOI: 10.1037/0033-2909.111.2.361.

Miller T. 2017. Explanation in Artificial Intelligence: Insights from the Social Sciences. arXiv:1706.07269 [cs].

Mitchell SD. 2002. Integrative Pluralism. Biology \& Philosophy 17:55-70. DOI: 10.1023/A:1012990030867.

Mitchell S. 2012. Unsimple truths: Science, complexity, and policy. Chicago, Mich.: The Univ. of Chicago Press.

Molenaar PCM. 2004. A Manifesto on Psychology as Idiographic Science: Bringing the Person Back Into Scientific Psychology, This Time Forever. Measurement: Interdisciplinary Research \& Perspective 2:201-218. DOI: $10.1207 / \mathrm{s} 15366359 \mathrm{mea} 0204 \_1$.

Molenaar PC, Campbell CG. 2009. The New Person-Specific Paradigm in Psychology. Current Directions in Psychological Science 18:112-117. DOI: 10.1111/j.1467-8721.2009.01619.x.

Molnar C. 2018. Interpretable Machine Learning. Leanpub.

Molnar C, Bischl B, Casalicchio G. 2018. Iml: An R package for Interpretable Machine Learning. JOSS 3:786. DOI: $10.21105 /$ joss.00786. 
Morey RD, Hoekstra R, Rouder JN, Lee MD, Wagenmakers E-J. 2016. The fallacy of placing confidence in confidence intervals. Psychonomic Bulletin $\& 3$ Review 23:103-123. DOI: 10.3758/s13423-015-0947-8.

Nevill AM, Williams AM, Boreham C, Wallace ES, Davison GW, Abt G, Lane AM, BOARD EMWE. 2018. Can we trust "Magnitude-based inference"? Journal of Sports Sciences 36:2769-2770. DOI: 10.1080/02640414.2018.1516004.

Norman GR, Gwadry Sridhar F, Guyatt GH, Walter SD. 2001. Relation of Distribution- and Anchor-Based Approaches in Interpretation of Changes in Health-Related Quality of Life: Medical Care 39:1039-1047. DOI: 10.1097/00005650-200110000-00002.

Novick MR. 1966. The axioms and principal results of classical test theory. Journal of Mathematical Psychology 3:1-18. DOI: 10.1016/0022-2496(66)90002-2.

O'Hagan T. 2004. Dicing with the unknown. Significance 1:132-133. DOI: 10.1111/j.1740-9713.2004.00050.x. Page SE. 2018. The Model Thinker: What You Need to Know to Make Data Work for You. Basic Books.

Pearl J. 2009. Causal inference in statistics: An overview. Statistics Surveys 3:96-146. DOI: 10.1214/09-SS057.

Pearl J. 2019. The seven tools of causal inference, with reflections on machine learning. Communications of the ACM 62:54-60. DOI: 10.1145/3241036.

Pearl J, Glymour M, Jewell NP. 2016. Causal Inference in Statistics: A Primer. Chichester, West Sussex: Wiley.

Pearl J, Mackenzie D. 2018. The Book of Why: The New Science of Cause and Effect. New York: Basic Books.

R Core Team. 2018. R: A Language and Environment for Statistical Computing. Vienna, Austria: R Foundation for Statistical Computing.

Reshef DN, Reshef YA, Finucane HK, Grossman SR, McVean G, Turnbaugh PJ, Lander ES, Mitzenmacher M, Sabeti PC. 2011. Detecting Novel Associations in Large Data Sets. Science 334:1518-1524. DOI: 10.1126/science.1205438.

Revelle W. 2018. Psych: Procedures for Psychological, Psychometric, and Personality Research. Evanston, Illinois: Northwestern University.

Ribeiro MT, Singh S, Guestrin C. 2016. "Why Should I Trust You?": Explaining the Predictions of Any Classifier. arXiv:1602.04938 [cs, stat].

Rohrer JM. 2018. Thinking Clearly About Correlations and Causation: Graphical Causal Models for Observational Data. Advances in Methods and Practices in Psychological Science 1:27-42. DOI: $10.1177 / 2515245917745629$.

Rousselet GA, Pernet CR, Wilcox RR. 2017. Beyond differences in means: Robust graphical methods to compare two groups in neuroscience. European Journal of Neuroscience 46:1738-1748. DOI: 10.1111/ejn.13610.

Rousselet GA, Pernet CR, Wilcox RR. 2019a. A practical introduction to the bootstrap: A versatile method to make inferences by using data-driven simulations. DOI: 10.31234/osf.io/h8ft7.

Rousselet GA, Pernet CR, Wilcox RR. 2019b. The percentile bootstrap: A teaser with step-by-step instructions in R. DOI: 10.31234/osf.io/kxarf.

RStudio Team. 2016. RStudio: Integrated Development Environment for R. Boston, MA: RStudio, Inc.

Saddiki H, Balzer LB. 2018. A Primer on Causality in Data Science. arXiv:1809.02408 [stat].

Sainani KL. 2012. Clinical Versus Statistical Significance. PMER 4:442-445. DOI: 10.1016/j.pmrj.2012.04.014.

Sainani KL. 2018. The Problem with "Magnitude-based Inference". Medicine and Science in Sports and Exercise 50:2166-2176. DOI: 10.1249/MSS.0000000000001645. 
Sainani KL, Lohse KR, Jones PR, Vickers A. 2019. Magnitude-Based Inference is Not Bayesian and is Not a Valid Method of Inference. Scandinavian Journal of Medicine 63 Science in Sports. DOI: 10.1111/sms.13491.

Savage LJ. 1972. The Foundations of Statistics. New York: Dover Publications.

Shmueli G. 2010. To Explain or to Predict? Statistical Science 25:289-310. DOI: 10.1214/10-STS330.

Shrier I, Platt RW. 2008. Reducing bias through directed acyclic graphs. BMC Medical Research Methodology 8. DOI: $10.1186 / 1471-2288-8-70$.

Swinton PA, Hemingway BS, Saunders B, Gualano B, Dolan E. 2018. A Statistical Framework to Interpret Individual Response to Intervention: Paving the Way for Personalized Nutrition and Exercise Prescription. Frontiers in Nutrition 5. DOI: 10.3389/fnut.2018.00041.

Turner A, Brazier J, Bishop C, Chavda S, Cree J, Read P. 2015. Data Analysis for Strength and Conditioning Coaches: Using Excel to Analyze Reliability, Differences, and Relationships. Strength and Conditioning Journal 37:76-83. DOI: 10.1519/SSC.0000000000000113.

Wagenmakers E-J. 2007. A practical solution to the pervasive problems ofp values. Psychonomic Bulletin $\&$ Review 14:779-804. DOI: 10.3758/BF03194105.

Watts DJ, Beck ED, Bienenstock EJ, Bowers J, Frank A, Grubesic A, Hofman J, Rohrer JM, Salganik M. 2018. Explanation, prediction, and causality: Three sides of the same coin? DOI: 10.31219/osf.io/u6vz5.

Weinberg G, McCann L. 2019. Super thinking: The big book of mental models. New York: Portfolio/Penguin. Welsh AH, Knight EJ. 2015. "Magnitude-based Inference": A Statistical Review. Medicine ES Science in Sports \&3 Exercise 47:874-884. DOI: 10.1249/MSS.0000000000000451.

Wickham H. 2017. Tidyverse: Easily Install and Load the 'Tidyverse'.

Wilcox RR. 2016. Introduction to robust estimation and hypothesis testing. Waltham, MA: Elsevier.

Wilcox R, Peterson TJ, McNitt-Gray JL. 2018. Data Analyses When Sample Sizes Are Small: Modern Advances for Dealing With Outliers, Skewed Distributions, and Heteroscedasticity. Journal of Applied Biomechanics 34:258-261. DOI: 10.1123/jab.2017-0269.

Wilcox RR, Rousselet GA. 2017. A guide to robust statistical methods in neuroscience. bioRxiv. DOI: $10.1101 / 151811$.

Wilke CO. 2018. Ggridges: Ridgeline Plots in 'ggplot2'.

Wilke CO. 2019. Cowplot: Streamlined Plot Theme and Plot Annotations for 'ggplot2'.

Xie Y. 2014. Knitr: A Comprehensive Tool for Reproducible Research in R. In: Stodden V, Leisch F, Peng RD eds. Implementing Reproducible Computational Research. Chapman and Hall/CRC,

Xie Y. 2015. Dynamic Documents with $R$ and knitr. Boca Raton, Florida: Chapman and Hall/CRC.

Xie Y. 2018. Knitr: A General-Purpose Package for Dynamic Report Generation in R.

Xie Y, Allaire JJ, Grolemund G. 2018. R Markdown: The Definitive Guide. Boca Raton, Florida: Chapman and Hall/CRC.

Yarkoni T, Westfall J. 2017. Choosing Prediction Over Explanation in Psychology: Lessons From Machine Learning. Perspectives on Psychological Science 12:1100-1122. DOI: 10.1177/1745691617693393.

Zhao Q, Hastie T. 2019. Causal Interpretations of Black-Box Models. Journal of Business E Economic Statistics:1-10. DOI: 10.1080/07350015.2019.1624293.

Zhu H. 2019. kableExtra: Construct Complex Table with 'kable' and Pipe Syntax. 